\title{
Nonlinear Response and Avalanche Behavior in Metallic Glasses
}

\author{
Dissertation \\ zur Erlangung des mathematisch-naturwissenschaftlichen \\ Doktorgrades \\ "Doctor rerum naturalium" \\ der Georg-August-Universität Göttingen \\ im Promotionsprogramm ProPhys \\ der Georg-August University School of Science
}

vorgelegt von

Birte Riechers

aus Wolfsburg

Göttingen, 2017 
Betreuungsausschuss

Prof. Dr. Konrad Samwer

I. Physikalisches Institut, Georg-August-Universität Göttingen

Prof. Dr. Hans-Ulrich Krebs

Institut für Materialphysik, Georg-August-Universität Göttingen

Mitglieder der Prüfungskommission

Referent:

Prof. Dr. Konrad Samwer

I. Physikalisches Institut, Georg-August-Universität Göttingen

Korreferentin:

Prof. Dr. Cynthia Volkert

Institut für Materialphysik, Georg-August-Universität Göttingen

Externer Gutachter:

Prof. Dr. Jörg Löffler

Departement Materialwissenschaft, Eidgenössische Technische Hochschule Zürich

Weitere Mitglieder der Prüfungskommission

Prof. Dr. Vasily Moshnyaga

I. Physikalisches Institut, Georg-August-Universität Göttingen

Prof. Dr. Michael Seibt

IV. Physikalisches Institut, Georg-August-Universität Göttingen

Dr. Richard Vink

Institut für Materialphysik, Georg-August-Universität Göttingen

Dr. Claus Heussinger

Institut für Theoretische Physik, Georg-August-Universität Göttingen

Tag der mündlichen Prüfung: 07. Juni 2017 
Für den Helden meines Alltags. 


\begin{abstract}
The response to different stress amplitudes at temperatures below the glass transition temperature is analyzed by mechanical oscillatory excitation of $\mathrm{Pd}_{40} \mathrm{Ni}_{40} \mathrm{P}_{20}$ metallic glass samples in single cantilever bending geometry. The strain response of the material is well below the critical yield stress even for highest stress amplitudes, implying the expectation of a linear relation between stress and strain according to Hook's Law. However, a deviation from the linear behavior is evident, which is evaluated in terms of temperature dependence and influence of the applied stress amplitude by two different approaches of evaluation.

The nonlinear approach is based on a nonlinear expansion of the stress-strain-relation, assuming an intrinsic nonlinear character of the shear or elastic modulus. The degree of nonlinearity is extracted by a period-by-period Fourier-analysis and connected to nonlinear coefficients, describing the intensity of nonlinearity at the fundamental and higher harmonic frequencies. While rather small stress amplitudes are connected to a linear response behavior, higher stress amplitudes result in nonlinear behavior, which is enhanced with increasing temperature. The characteristic timescale to adapt to a significant change in stress amplitude in terms of a recovery timescale to a steady state value is connected to the structural relaxation time of the material, suggesting a connection between the observed nonlinearity and primary relaxation processes.

The second approach of evaluation is termed the incremental analysis and relates the observed response behavior to avalanches, which occur due to the activation and correlation of local microstructural rearrangements consisting of a few tens of atoms. These rearrangements are termed as shear transformation zones and correspond to localized plastic events, which are superimposed on the linear response behavior of the material. Temperature and stress enhance the occurrence of intervals of monotonously increasing or decreasing strain. These are connected to avalanche behavior according to the power-law observed in the distributions of strain response. Despite the onset of nonlinearity observed in connection with the nonlinear analysis, the power-law behavior is extracted for all applied stress amplitudes, high and low, suggesting activated plastic events throughout the so called Hookean response regime. The intensity of strain response itself shows a direct relation to the strain rate of the experiment.

Both approaches of evaluation are compared regarding common aspects and limits. Response phenomena connected to plastic events in the linear response regime are barely reported in literature even though their existence is implied. The incremental analysis yields experimental evidence supporting their occurrence. Moreover it reflects the limits of the nonlinear approach, which neglects strain response on the small scale by the period-wise analysis of the data, resulting in an apparent linear strain response. Still, the nonlinear approach illustrates clearly on a global scale, that stress and temperatures have a similar effect of pushing the material further towards the yielding transition and thus a more compliant behavior.
\end{abstract}




\section{Contents}

Abstract ix

$\begin{array}{lll}1 & \text { Introduction } & 1\end{array}$

$\begin{array}{lll}2 & \text { Theoretical Framework } & 3\end{array}$

2.1 The Glass Transition . . . . . . . . . . . . . . . . . . . . . . . . . . . . . . . . 4

2.2 Fragility of Amorphous Systems . . . . . . . . . . . . . . . . . . . 6

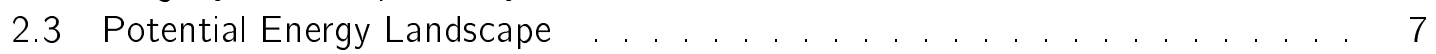

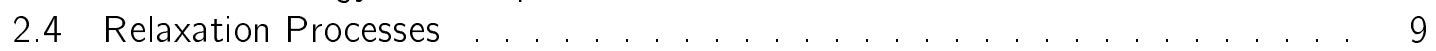

2.5 Microscopic Description of Rearrangements . . . . . . . . . . . . . . . . . 12

2.6 Influence of Mechanical Excitation . . . . . . . . . . . . . . . . . . . . . . . . . . 18

2.7 Response to High Field Excitations . . . . . . . . . . . . . . . . . . . . . . 21

$2.7 .1 \quad$ Nonlinear Response in Dielectric Spectroscopy . . . . . . . . . . . . 23

2.7 .2 Linear Response and Instantaneous Breakdowns of Elasticity . . . . . 28

3 Experimental and Analytical Methods 33

3.1 Preparation of Metallic Glass Samples . . . . . . . . . . . . . . . . . . 33

3.2 X-Ray Diffraction . . . . . . . . . . . . . . . . . . . . . . . . . . . . . . 34

3.3 Energy Dispersive X-ray Spectroscopy . . . . . . . . . . . . . . . . . . . . . . 34

3.4 Differential Scanning Calorimetry . . . . . . . . . . . . . . . . . . . . . . 35

3.5 Dynamical Mechanical Analysis . . . . . . . . . . . . . . . . . . . . 36

3.5 .1 Temperature Scans and Static Stress-Strain-Curves . . . . . . . . 38

3.5 .2 Large Amplitude Oscillatory Spectroscopy . . . . . . . . . . . . . . . 38

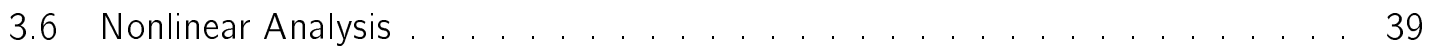

3.6 .1 Nonlinear Response to Mechanical Excitation . . . . . . . . . . . . . 39

3.6 .2 Fourier Analysis of LAOS Measurements . . . . . . . . . . . . . . . 41

3.6 .3 Fit of Time-Dependent Behavior . . . . . . . . . . . . . . . . . . . . 41

3.7 Incremental Analysis . . . . . . . . . . . . . . . . . . . . . . . . . . 42

$3.7 .1 \quad$ Extraction of Positive Ascending Part of each Period . . . . . . . . . 43

3.7 .2 Definition of Increments and Intervals . . . . . . . . . . . . . . 43

3.7 .3 Generation of Double-Logarithmic Distributions. . . . . . . . . . . . 46

\begin{tabular}{|lll}
\hline & Sample Characterization & $\mathbf{4 7}$
\end{tabular}

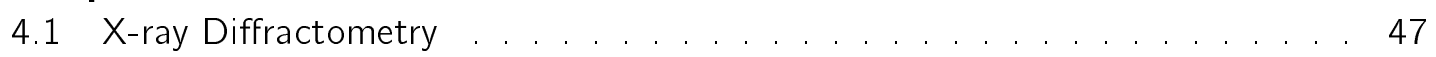

4.2 Energy Dispersive X-ray Spectroscopy . . . . . . . . . . . . . . . . . . . . . . 48

4.3 Differential Scanning Calorimetry . . . . . . . . . . . . . . . . . . . . . . . . . . 48

4.4 Static Stress-Strain-Curves . . . . . . . . . . . . . . . . . . . . . . . . . . . . . . . . . . . 49

4.5 Dynamical Mechanical Temperature Scans . . . . . . . . . . . . . . . . 50

5 Results on LAOS Measurements

5.1 Lissajous-Plots . . . . . . . . . . . . . . . . . . . . . . . . . . . . . . 54

5.2 Comparison of LAOS-Measurements to Temperature Scans . . . . . . . 57

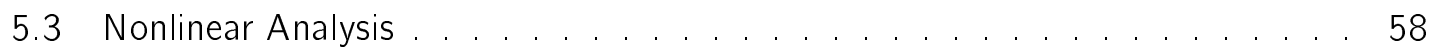


5.3 .1 Storage Compliance at Fundamental Frequency . . . . . . . . . . . . 59

5.3 .2 Loss Compliance at Fundamental Frequency . . . . . . . . . . . . . 63

5.3 .3 Compliance at Higher Harmonic Frequency . . . . . . . . . . . . . . 67

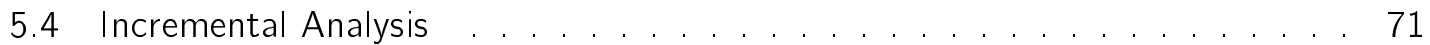

5.4 .1 Strain Rate . . . . . . . . . . . . . . . . . . . . . . . . 72

5.4 .2 Averaged Values for Strain Intervals . . . . . . . . . . . . . . . 74

5.4 .3 Distributions of Strain Intervals . . . . . . . . . . . . . . . . . . . 82

\begin{tabular}{lll}
\hline 6 & Discussion & $\mathbf{8 7}$
\end{tabular}

6.1 Characterization of Sample Properties . . . . . . . . . . . . . . . . . 87

6.2 Qualitative Response Behavior . . . . . . . . . . . . . . . . . . . . . . 87

6.3 Nonlinear Response Behavior . . . . . . . . . . . . . . . . . . . . . . . . . 88

6.4 Incremental Response Behavior . . . . . . . . . . . . . . . . . . . . . . . . . . 95

6.5 Comparison and Classification of Results . . . . . . . . . . . . . . . . . 101

6.6 Outlook . . . . . . . . . . . . . . . . . . . . . . . . . . . . 103

\begin{tabular}{|lr}
\hline A Calibration of the DMA 8000 & 109
\end{tabular}

\begin{tabular}{ll}
\hline B Geometry Factor & 111
\end{tabular}

\begin{tabular}{|r|r|}
\hline C Sinusoidal Excitation Including Offset & 112
\end{tabular}

\begin{tabular}{|lr|}
\hline D Fifth Harmonic Frequency Response & 114
\end{tabular}

\begin{tabular}{llr}
\hline E & MatLab-Routines for Incremental Analysis & $\mathbf{1 1 5}$
\end{tabular}

E.1 Definition of Increments . . . . . . . . . . . . . . . . . . . . . 115

E.2 Definition of Intervals . . . . . . . . . . . . . . . . . . . . . . . . . . 118

E.3 Subdivision to Stress-Windows . . . . . . . . . . . . . . . . . . 120

E.4 Extraction of Strain Interval Distributions . . . . . . . . . . . . . . . . 130

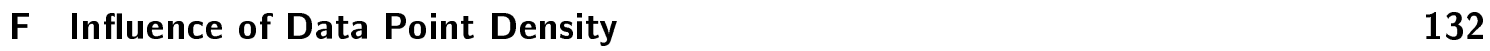

\begin{tabular}{lll}
\hline G Distributions on ideal Sinusoidal Data & 134
\end{tabular} 


\section{Chapter 1}

\section{Introduction}

Amorphous materials span over a huge variety of classes, including network glasses, e.g. $\mathrm{SiO}_{2}$ [38], polymers, such as polymethylmethacrylate [44] or polycarbonate [58], sugars and alcohols, for example glycerol [148] and propylene glycol [148], metallic glasses like $\mathrm{Pd}_{40} \mathrm{Ni}_{40} \mathrm{P}_{20}$ [125, [151, 67], plastic crystals, e.g. neopentylglycol [118] or cyclo-octanol [93], and many more. All of these materials have in common that under specific preparation conditions or even naturally an amorphous order regarding their structure is exhibited. In case of structural glass-formers, the molecules or atoms of the material are arranged in a way that allows for short-range order, but it is elusive of long-range order [38].

The preparation techniques to obtain amorphous materials are numerous, among them sputtering [156], pulsed laser deposition [123], and melt quenching [75]. The melt quenching technique can be applied to prepare samples of bulk metallic glass. Therefore the melt of an alloy of desired composition is cooled with such a high cooling rate that crystallization is avoided. The melt traverses the melting point without immediate solidification resulting in a supercooled melt. By further rapid decrease in temperature, particle motion becomes more and more kinetically hindered, until the sample is referred to as being in the glassy state. In simple words, the particles get stuck in their positions due to the very quick cooling, before the atoms or molecules have enough time to order into a crystalline state.

At temperatures close to or above the glass transition temperature, the response to the excitation by a small external field on a sufficiently small timescale results in the measurement of relaxations. These correspond to structural rearrangements of the constituents of the amorphous material. The activation and correlation of structural rearrangements result in the characteristic response behavior, which can be measured for example by mechanical excitation. The more relaxations become active in the material, the more intense is the response to an applied field. To understand how these structural relaxations occur and moreover how they correlate on the microscale is highly relevant to be able to understand the macroscopically observed behavior.

This macroscopic relation between an applied force and the corresponding displacement of the sample is given by the stress-strain-curve. Its slope is identified with the mechanical susceptibility, referred to as the modulus. It is constant for small mechanical excitations, but approaches zero under intense mechanical load. On this macroscopic, i.e. global scale, the modulus is intrinsically nonlinear, as reflected by the deviation from the constant modulusvalue if an elevated stress is applied.

In 1965 Adam and Gibbs proposed the idea of cooperative rearranging regions [1], which laid the basis for the interpretation of deformation in amorphous materials. These were developed to the picture of shear transformation zones as proposed by Argon [5, 6, 129]. Simplified, STZs are depicted as the basis of mechanical deformation in amorphous metals, which exhibit a correlation in space and time [82, 122]. Depending on the interplay between stress and temperature, these correlations can evolve to shear bands, which become system-spanning events and eventually lead to sample failure, similar as observed for crystalline materials. 
As stated before, the analysis of the interplay between microscopic rearranging processes is the basis for the understanding of the mechanical deformation in amorphous materials. The identification of the highly nonlinear part of the stress-strain-curve at elevated stresses as the plastic regime which exhibits macroscopic shear bands is one important step. However, the converse argument that the linear response regime is solely connected to elastic response behavior is challenged in literature [77]. The linear response regime exhibits events of plastic character as well, even though much less pronounced than the case during shear band formation. To elucidate the response characteristics of the so called linear mechanical response regime of metallic glasses, $\mathrm{Pd}_{40} \mathrm{Ni}_{40} \mathrm{P}_{20}$-samples are analyzed regarding nonlinear response aspects and indications for the occurrence of STZs.

In order to present the various aspects of this intention, chapter 2 evolves on theoretical aspects and models regarding the amorphous state, relaxation processes and their interpretation. Chapter 3 summarized the methods used for sample characterization and techniques for the mechanical measurements. It moreover gives a detailed description on the two different analysis approaches, i.e. the nonlinear and the incremental analysis. The results on the sample characterization are given in chapter 4 , while the results on the mechanical experiments themselves are presented in chapter 5. These are separated into several sub-chapters, which are on the qualitative sample response (section 5.1 and 5.2), the quantitative sample response based on the nonlinear analysis (section 5.3), and results on the quantitative sample response based on the incremental analysis (section 5.4). A discussion of the results and a critical examination of the connection of the different analysis approaches are given in chapter 6 . 


\section{Chapter 2}

\section{Theoretical Framework}

This chapter gives an overview of the topics relevant for the experimental approach of this thesis based on literature research. It reflects personal insights and scientific interrelations gained during the time spent on the development of the thesis with Prof. Konrad Samwer, on projects with Prof. Ranko Richert and Prof. Itamar Procaccia, and by the participation in a number of national and international conferences confronting with present scientific questions of the field.

Initially, it covers the description of how to attain an amorphous state, and of the nature of the glass transition. Evidently, different types of processes dominate in the glass and in the supercooled liquid. This is addressed by discussing the change of activation energies with temperature, followed by the classification into fragile and strong glass formers, and an introduction to the potential energy landscape model. It serves as a helpful, even though limited model to explain temperature and stress dependent behavior and differentiate between different relaxation types. Primary and secondary relaxations are described due to their evolution in loss spectra, as well as on the micro-structural scale. They are connected to reversible and irreversible processes based on shear transformation zones and thus Eshelby-type elastic interactions. The influence of mechanical excitation on the processes is illustrated with help of an experimental stress-strain-measurement, introducing shear-bands and their connection to the primary relaxation process. Further on, the implication of high field excitation is discussed with regard to the influence on the glass transition temperature and the mechanical yielding transition. It is followed by a more detailed description on dielectric spectroscopy measurements exemplifying the experimental approach of this thesis, which is elucidated in the successive sections. Finally, this nonlinear approach is opposed to the consideration of the high-field response as a state that can be connected to avalanche dynamics based on the evaluation of instabilities in the elastic behavior, referring to statistical, localized breakdowns of elasticity. 


\subsection{The Glass Transition}

When the temperature of a liquid is decreased, the path of solidification depends highly on the kinetics of the material and the cooling rate.

From a thermodynamic point of view a system with mobile atoms or molecules will crystallize, if the free enthalpy of the solidus line becomes smaller than the one of the liquidus line while cooling. This crystallization will occur at a temperature $T_{S}$ (see fig. 2.1a) under sudden contraction to a solid exhibiting order both on short and long ranges.

Another route leads to a solidification into the amorphous phase. It can be addressed by realizing very high cooling rates (e.g. $10^{5} \mathrm{Ks}^{-1}$ for metallic glasses [51]), especially in materials of low atomic or molecular mobility. Due to the quick cooling the viscosity is increased sufficiently fast to hinder the development of long-range order. The material is called a supercooled liquid as long as the system pursues the liquidus line even though the temperature is below $T_{S}$. If the temperature is decreased further and the system relinquishes the supercooled liquid state it falls out of equilibrium and becomes a glass, the metastable state of an amorphous solid. In a plot of volume $V$ or entropy $S$ versus temperature $T$, the temperature range of changing slope is called the glass transition range. The temperature at which the supercooled liquid regime is abandoned is called the glass transition temperature $T_{g}$. The temperature at which an extrapolation of the glass and the supercooled liquid curve intersect is the fictive temperature $T_{f}$ [43]. $T_{g}$ (as well as $T_{f}$ ) is highly dependent on the cooling rate. Different cooling rates result in different branches of the glass. These branches separate at higher temperatures from the supercooled liquid with larger cooling rates, thus $T_{g 1}$ is gained by faster cooling than $T_{g 2}$ (cf. fig. 2.1a). The volume for a glass is roughly $1 \%$ higher than in the crystalline state, even though the slope in the plot against temperature is comparable. The heating rate has an influence on the glass transition as well, if the system is heated from the glassy state to temperatures close to $T_{g}$ : The temperature at which the system gets back into the supercooled liquid state is lower, if the heating rate is small. Holding a glass at temperatures not lower than $50 \mathrm{~K}$ to $100 \mathrm{~K}$ from $T_{g}$ gives the system the chance to relax on a certain timescale [90] to a state of less volume $\left(T_{1}\right)$, thus decreasing the fictive temperature. This relaxation process is also called aging, as it occurs with time and endures longer for low the temperatures. It can be in the range of several minutes slightly below $T_{g}$, or hundreds of years far below the glass transition temperature. In this sense it is also possible to rejuvenate a glass, e.g. by radiation. Volume can be introduced into the material and the

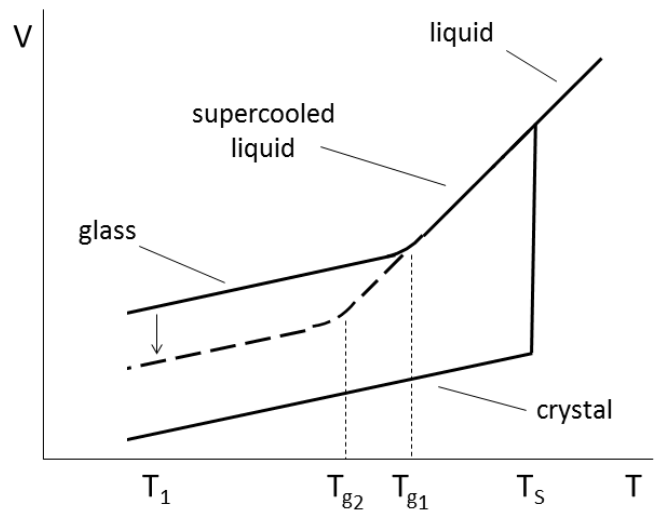

(a)

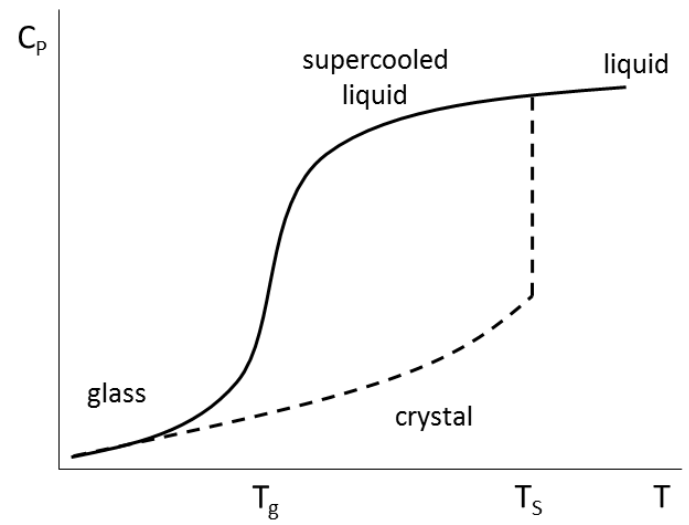

(b)

Figure 2.1 (a) Volume $V$ plotted against temperature $T$. (b) Specific heat $C_{P}$ plotted against temperature $T$. Both giving a schematic example for the first order phase transition from the liquid to the crystalline state, and the transition from the liquid, via the supercooled liquid, to the glassy state, cf. [38. 
entropy is thereby increased. The resulting rejuvenated state is connected to a higher fictive temperature.

The glass transition itself is not considered a thermodynamic phase transition, even though it was suggested that it might be a second-order phase transition according to Ehrenfest's scheme [37]. Here, the order of the phase transition is defined as the lowest derivative of the Gibb's free energy $F_{G}$ which shows a discontinuity at the transition point [38]. In glasses, the second derivative of Gibbs free energy $F_{G}$ shows a discontinuity in differential quantities, which is e.g. the specific heat $C_{P}$. However, the strong dependence on thermal history and cooling rate of the glassy state and the glass transition itself stands in contradiction to this categorization. Fig. 2.1b shows schematically the transition from the glassy state at low temperatures to the supercooled liquid. For glass and crystal the specific heat $C_{P}$ is dominated by vibrational contributions and shows thus comparable values $\left(C_{P}^{\text {glass }} \approx C_{P}^{\text {crystal }}\right)$. For the supercooled liquid state the specific heat exceeds the value of the crystal. Configurational degrees of freedom dominate in this regime, which are not accessible in the crystalline state.

$$
\begin{gathered}
S=-\left(\frac{\partial F_{G}}{\partial T}\right)_{P} \\
C_{P}=\left(\frac{\partial F_{G}}{\partial T}\right)_{P}^{2}
\end{gathered}
$$

Another option to define the glass transition temperature is connected to the viscosity of the system. A viscosity of $10^{12} \mathrm{~Pa} \mathrm{~s}$, equivalent to a relaxation timescale of $10^{2} \mathrm{~s}$, is chosen to be the definition of the glass transition. This is a value, at which a system becomes measurable on the laboratory timescale. As an example, a glass sample of $1 \mathrm{~cm}$ thickness, bonded to a vertical plane surface will flow on a measurable scale due to gravity within several month, if the viscosity drops slightly below the value used to define the glass transition temperature [68].

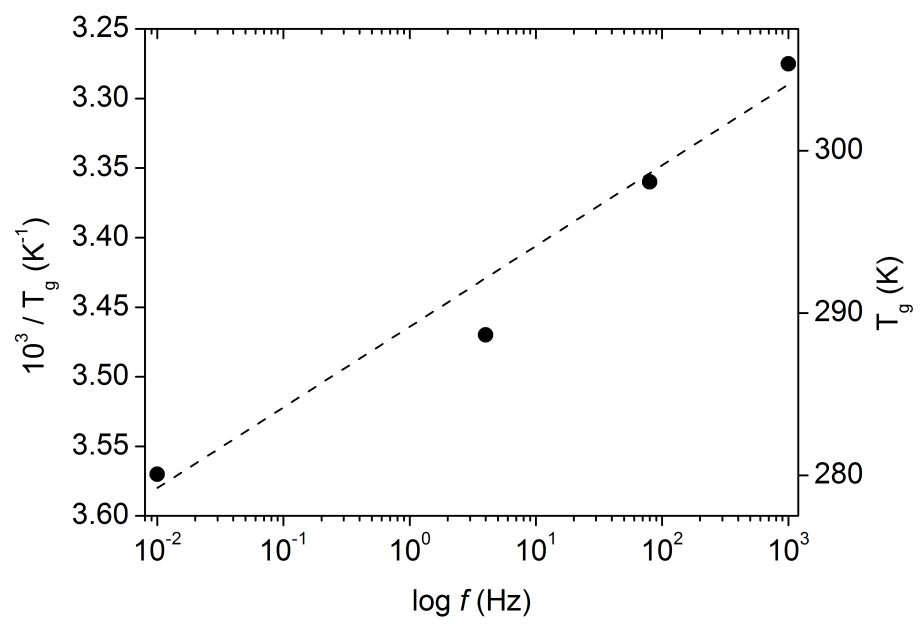

Figure 2.2 Plot of the glass transition temperature $T_{g}$ against frequency $f$ connected to the experimental timescale for poly-3:3-biscloromethyloacyclobutane. Experimental techniques are dielectric spectroscopy (dielectric loss, $f=10^{3} \mathrm{~Hz}$ ), mechanical vibration (mechanical loss, $f=89 \mathrm{~Hz}$ ), slow tensile deformation $(f=3 \mathrm{~Hz})$, and dilatometry $\left(f=10^{-2} \mathrm{~Hz}\right)[38$ ].

The glass transition temperature $T_{g}$ depends highly on the chosen protocol and varies with different measurement techniques as the timescale of the experiment changes. In fig. 2.2. $T_{g}$ is plotted against the experimental timescale which is measured by four different measurement techniques.

The configurational changes that occur explicitly in the supercooled liquid cause the relaxation of the system. When the temperature of the system decreases, these relaxations become slower. At a given temperature, which is the glass transition temperature $T_{g}$, the material 


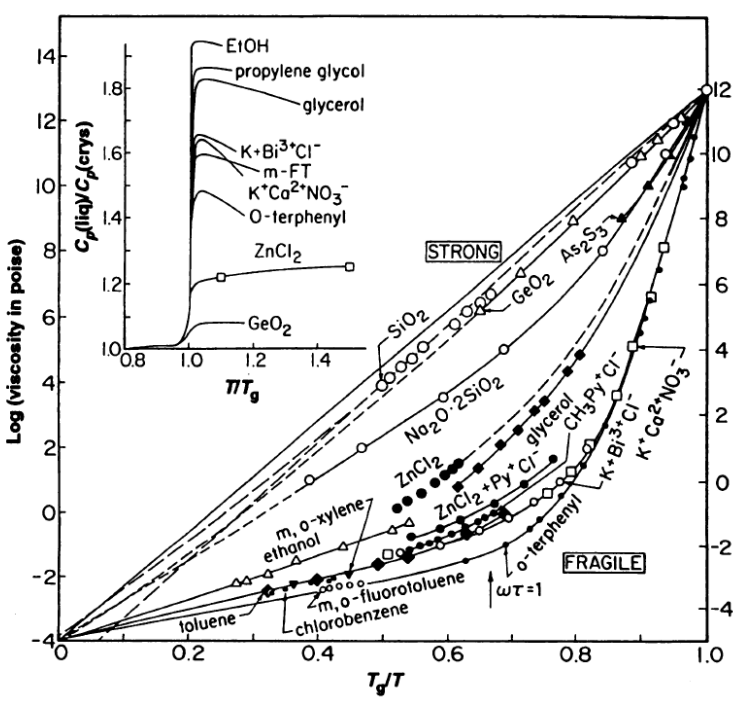

(a)

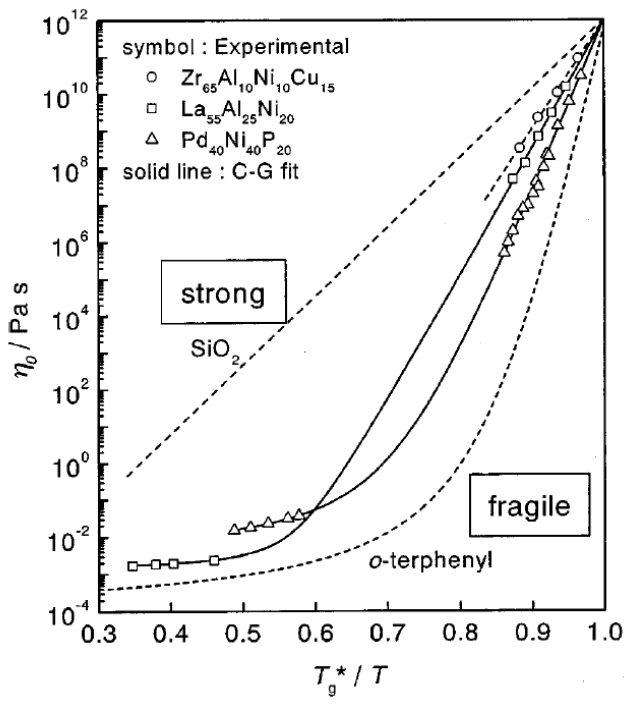

(b)

Figure 2.3 Angell-plots of viscosity $\eta$ against normalized, inverse temperature $T_{g} / T$ for different amorphous systems. (a) According to [4]. (b) According to [67].

shows the behavior of a solid [38]. If the experimental timescale or observation time $t_{0}$ is long compared to the relaxation timescale $\tau_{R}\left(t_{0}>\tau_{R}\right)$, the material shows a liquid-like behavior. With an observation time being smaller than the relaxation timescale $\left(t_{0}<\tau_{R}\right)$, the material becomes solid-like. In this regard, the glass transition occurs at the temperature at which both timescales are of comparable order of magnitude $\left(t_{0} \approx \tau_{R}\right)$.

\subsection{Fragility of Amorphous Systems}

The glass transition regime exhibits a rapid, but continuous change of the viscosity $\eta$. The way how the viscosity changes with temperature is used to separate amorphous materials as fragile or strong, as displayed in the Angell-plots [4, 67].

Strong behavior is often observed for network formers, e.g. $\mathrm{SiO}_{2}$, and their viscosity can be described by an Arrhenius-like description (eq. 2.2). It is characterized by energy barriers $E_{A}$, which do not show an intrinsic dependence on temperature. These barriers have to be overcome to initiate relaxation processes. The term $k_{B} T$ is due to the materials thermal energy, with Boltzmann constant $k_{B}$.

$$
\eta=\eta_{0} \cdot \exp \left(\frac{E_{A}}{k_{B} T}\right)
$$

Systems of fragile behavior are typically to be found in the class of organic or ionic glasses. Their behavior shows an intense deviation from the Arrhenius-law especially for temperatures clearly above $T_{g}$, as energy barriers depend on temperature. The Vogel-Fulcher-Tammann (VFT) fequation [143, 46, 136] is used to describe this behavior, based on the strength parameter $D$ and $T_{V F T}$ :

$$
\eta=\eta_{0} \cdot \exp \left(\frac{D \cdot T_{V F T}}{T-T_{V F T}}\right)
$$

The fragility index $m$ is introduced as a measure for the temperature-dependent viscosity of a material for temperatures in the glass transition regime [12]:

$$
m=\left.\frac{d \log \eta}{d\left(T_{g} / T\right)}\right|_{T=T_{g}}
$$


The stronger a system is, the smaller the fragility tends to be. The range of values starts from 20 for extremely strong glasses as $\mathrm{SiO}_{2}$ up to 200 for extremely fragile systems in the liquid extreme. $\mathrm{Pd}_{40} \mathrm{Ni}_{40} \mathrm{P}_{20}$ metallic glass can be classified as relatively fragile with a fragility index of $m=41.5$ [125], as shown in fig. 2.3b.

The fragility index is related to the strength parameter $D$ by [14]

$$
m=(D / \ln 10) \cdot\left(T_{0} / T_{g}\right) \cdot\left(1-T_{0} / T_{g}\right)^{-2}=16+590 / D .
$$

Adam and Gibbs proposed a connection between configurational entropy $S_{c}$ and the relaxation behavior [1]. They consider the mass transport in the system, e.g. diffusion or viscosity, as a result of cooperative atomic or molecular rearrangements. By finding the smallest size of a group of particles that is capable of performing a rearrangement at a temperature $T$, the probability of rearrangements and thus the viscosity can be expresses as a function of configurational entropy $S_{c}\left[38\right.$. $B$ and $\eta_{0}$ are denoted as constants.

$$
\begin{gathered}
\eta=\eta_{0} \cdot \exp \left(\frac{B}{T S_{C}}\right) \\
S_{c}=\int_{T_{0}}^{T} \Delta C_{P} d \ln T
\end{gathered}
$$

If the specific heat of an amorphous material is considered as small, the configurational entropy is almost independent of temperature and yields an Arrhenius-like dependence of viscosity on temperature. If in contrast the specific heat is large, the configurational entropy depends considerably on temperature and shows therefore a rather fragile behavior.

An analytical description on the dependence of the viscosity $\eta$ and thus the fragility $m$ on temperature $T$ was recently established for metallic glasses [73, 76, 72]. Within this approach, the effective atomic potential of a metallic glass is mapped onto a more simple expression which contains the parameter $\lambda$, describing the steepness of the repulsive part of the potential. It is extracted by fitting the short-range ascending slope of the radial distribution function $g(r)$ and is the only free fit-parameter to yield the relation between shear modulus and temperature $G(T)$ :

$$
G(T)=G\left(T_{g}\right) \cdot \exp \left[\alpha_{T} \cdot T_{g}(2+\lambda)\left(1-T / T_{g}\right)\right]
$$

Here, $G\left(T_{g}\right)$ is the shear modulus value at the glass transition, which can be expressed analytically, and $\alpha_{T}$ is the Debye-Grüneisen thermal expansion coefficient, which reflects the anharmonicity of the potential. On the basis of the activation energy $E(T)$ for local cooperative rearrangements and the cooperative shear model (cf. section 2.5), the analytical connection between viscosity $\eta$ and temperature $T$ can be derived, which is of double-exponential nature:

$$
\frac{\eta(T)}{\eta_{0}}=\exp \left[\frac{V_{c} \cdot G\left(T_{g}\right)}{k_{B} T} \cdot \exp \left((2+\lambda) \alpha_{T} T_{g}\left(1-T / T_{g}\right)\right)\right]
$$

$V_{c}$ represents the characteristic atomic volume, $k_{B}$ is the Boltzmann constant, and $\eta_{0}$ is a normalization constant. This equation can be connected to the fragility $m$ according to eq. 2.4. This analytical approach thus presents itself as a powerful tool to extract the dependence of shear modulus $G$, viscosity $\eta$, and fragility $m$ on the basis of only two parameters, $\lambda$ and $\alpha_{T}$, which reflect the repulsive and the attractive part of the effective inter-atomic potential.

\subsection{Potential Energy Landscape}

Based on the work of Goldstein [48] and others [3, 133], the description of a topographic multidimensional potential energy landscape (PEL) is established, that facilitates the understanding of relaxation processes in amorphous matter. The interaction of the system is 
captured by the potential energy function $\Phi\left(\vec{r}_{1} \ldots \vec{r}_{N}\right)$ based on the spatial location $\vec{r}_{i}$ of each particle. The elevation of $\Phi$ at any location $\vec{R} \equiv\left(\vec{r}_{1} \ldots \vec{r}_{N}\right)$ in configuration space of the $N$ particle system stands for the potential energy, containing minima, maxima, and saddle points similar to a topographic map of a landscape. This surface contains many minima of various depths, which are arranged in a complex pattern. Each minimum correlates to a mechanically stable arrangement of the $\mathrm{N}$ particles in space. Any (small) displacement results in restoring forces acting on the system's particles. The lowest lying minima became occupied if the system were cooled to zero temperature slowly enough to stay in thermal equilibrium, resulting in the state of an ideal crystal. Higher lying minima are related to amorphous states of the system, equivalent minima can be obtained by a permutation of identical particles. Adjacent basins, also referred to as inherent structure or inherent state, share a common saddle point, called a transient state (cf. fig. 2.4).

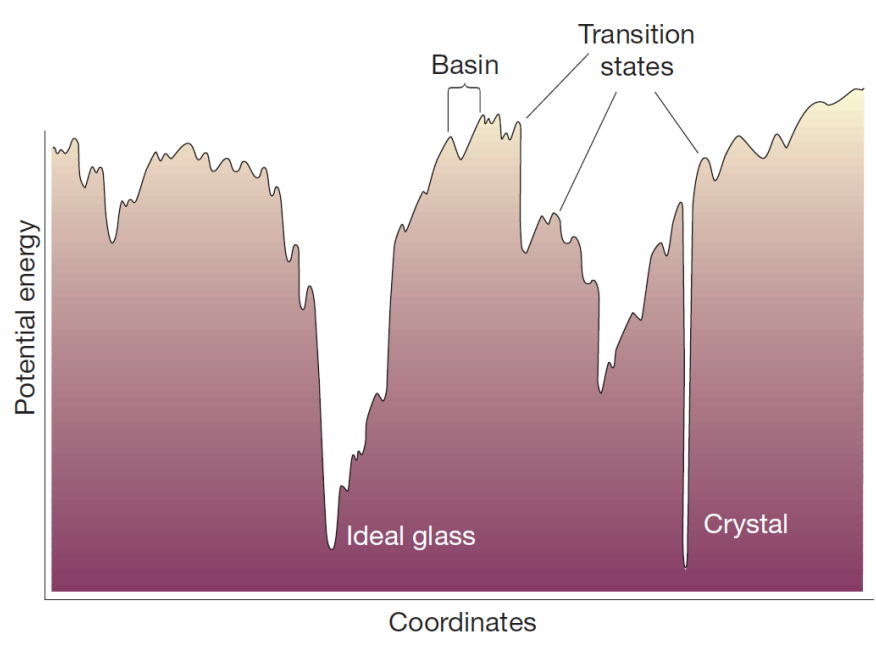

Figure 2.4 Scheme of the potential energy landscape [27].

The equilibrium of the system at a given temperature $T$ is connected to a preferential occupation of basins of depth $\Phi^{*}(T)$. For this reason the exploration of configuration space depends on temperature. A change from one basin to an adjacent one is realized by localized structural rearrangements of very few particles, going along with overcoming an activation barrier. The total amount of kinetic energy in the system is in general sufficiently high to pass this barrier, but the kinetic energy is distributed over all particles of the system. Thus, the lower the temperature, the lower the probability that the necessary kinetic energy is available in the required location. Only under this condition a proper fluctuation occurs by overcoming the transient state between basins. A hindrance of the hopping over barriers at low temperatures results in longer relaxation times. At temperatures typical for the supercooled state the system is in quasi-equilibrium, where it occupies and switches between minima with depths close to $\Phi_{\text {liq }}^{*}(T)$. With decreasing temperature, after entering the glassy state, the system falls out of equilibrium: Within a time smaller than the timescale necessary to change between minima, the depth of occupied basins still corresponds to values of $\Phi_{\text {liq }}^{*}(T)$ the system fails to proceed to minima of lower depth. This is a description that suits the formerly mentioned concept of fictive temperature, the rate dependence of the glass transition, and the ageing process in the glassy state.

Due to the direct connection between the activation energy $E_{A}$ regarding Arrhenius- and non-Arrhenius-behavior and the depth of basins in the $\mathrm{PEL}$ conclusions on the topography of the $\mathrm{PEL}$ can be made regarding the fragile or strong character of a material.

In case of a strong glass the activation energy is almost independent of temperature. Thus, regardless at which temperature the system samples the $\mathrm{PEL}$ if one barrier can be overcome, essentially all existing barriers can be overcome. This can only be the result of homogeneously distributed basin depths and thus a homogeneous distribution of activation energies. 
For fragile glasses at temperatures below $T_{g}$ only those parts of the PEL can be explored, which consist of adjacent basins which are less deep than $\Phi_{\text {liq }}^{*}(T)$. Only if the temperature rises above $T_{g}$, the depth of explorable basins increases and further parts of the PEL can be explored. Barriers can be overcome which are related to larger activation energies. These are due to cooperative rearrangements of a higher number of molecules than in the case of temperatures below $T_{g}$. Thus, the distribution of basin depths must be heterogeneous, including several metabasins, as depicted in fig. 2.5. Moreover, fragile materials should dispose a higher density of basins as their specific heat and thus their configurational entropy is high compared to strong materials [3, 4].

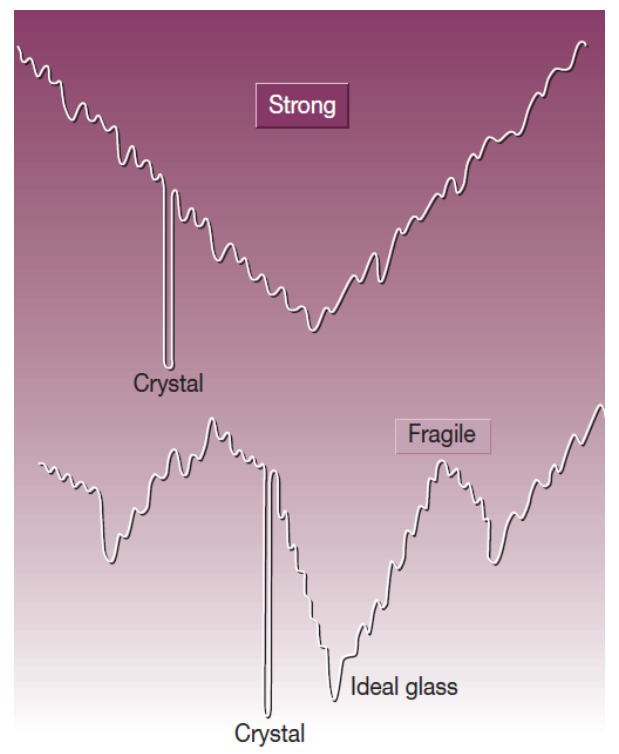

Figure 2.5 Schematic description of differences in the potential energy landscape for fragile and strong materials [27].

\subsection{Relaxation Processes}

To measure the configurational degrees of freedom, a relaxation response function can be obtained by a weak external excitation, which can be mechanical, electrical, thermal, or of other kind. Either the decay of the response to the exciting field is measured as a function of time, or the continuous dissipation due to an external sinusoidal excitation is detected. This way the relaxation response function $g$ depends either on time $t$ or frequency $f$. It reveals distinct processes, which occur on a very broad range of timescales.

At very short timescales intra-basin relaxations are active (not depicted in fig. 2.6a). These are of the order of vibrational movements and are collectively referred to as the boson peak. It occurs at frequencies between $10^{10} \mathrm{~Hz}$ and $10^{14} \mathrm{~Hz}$ in fig. 2.7. For larger timescales a very broad relaxation regime follows, that is due to inter-basin relaxations. It is depicted in fig. 2.6a as the $\beta$-process, also referred to as the secondary process. According to [55], the $\beta$ process might also be related to transitions between a finite number of inherent states in the sense of a seesaw hopping instead of only one transition from one basin to the next. In the frequency spectrum in fig. 2.7 secondary processes are found at frequencies between $10^{0} \mathrm{~Hz}$ and $10^{9} \mathrm{~Hz}$ for $T_{1}$ and between $10^{4} \mathrm{~Hz}$ and $10^{9} \mathrm{~Hz}$ for $T_{2}$. Whether they show the nature of a flank or a separated peak depends on temperature and the constituents of the amorphous system [160]. The $\beta$-relaxations overlap with the long-time limit, which is connected to relaxations between metabasins. These are depicted as the $\alpha$ - or primary process in fig. 2.6a and develop a relaxation peak at a frequency of $10^{1} \mathrm{~Hz}$ or $10^{4} \mathrm{~Hz}$ for 


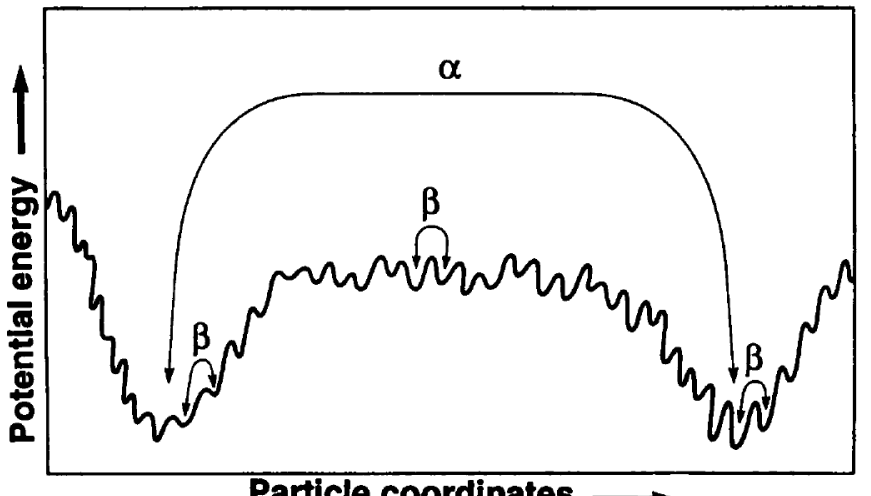

Particle coordinates

(a)

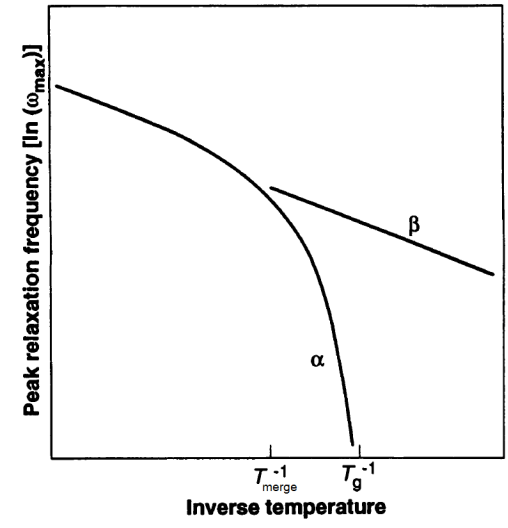

(b)

Figure 2.6 (a) Scheme of the potential energy landscape focusing on $\alpha$ - and $\beta$-relaxations. Changes between adjacent basins correspond to $\beta$-processes, while the $\alpha$-process is referred to as a transition from one metabasin to another. (b) Scheme of the peak relaxation frequency against inverse temperature for both primary and secondary relaxation processes [133].

high and low temperature, respectively (fig. 2.7). The position of the $\alpha$-relaxation peak is generally close to the mean relaxation time of the system and shifts according to the VFT. equation (2.3). The peak relaxation frequencies of the $\alpha$ - and the $\beta$-relaxation often show a bifurcation with temperature as in fig. 2.6b. At a temperature above $T_{g}$, namely the merging temperature $T_{\text {merge }}$, the branches of the peak relaxation frequencies of $\alpha$ - and $\beta$-processes join. Thus, for temperatures above $T_{\text {merge }}$ in the supercooled liquid as well as in the liquid range both relaxation processes occur on the same timescale. For temperatures close to and below $T_{\text {merge }}$, the primary and secondary relaxation is split in the time- and frequencydomain into two different maxima, of which the primary relaxation kinetically freezes out with decreasing temperature while the secondary persists [62].

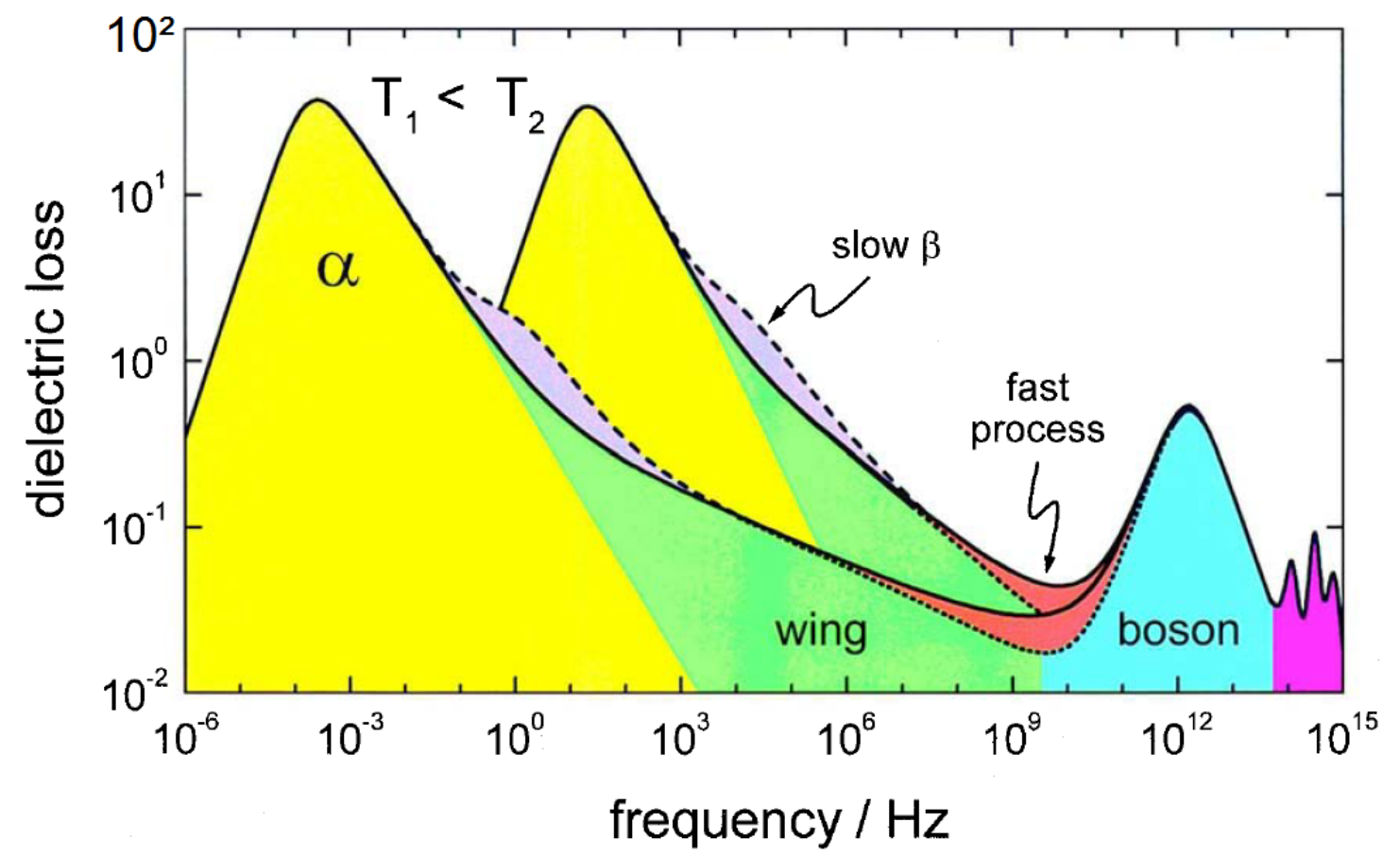

Figure 2.7 Schematic plot of dielectric loss versus frequency for glass-formers obtained by broadband spectroscopy for temperatures $T_{1}$ and $T_{2}$ [85]. 


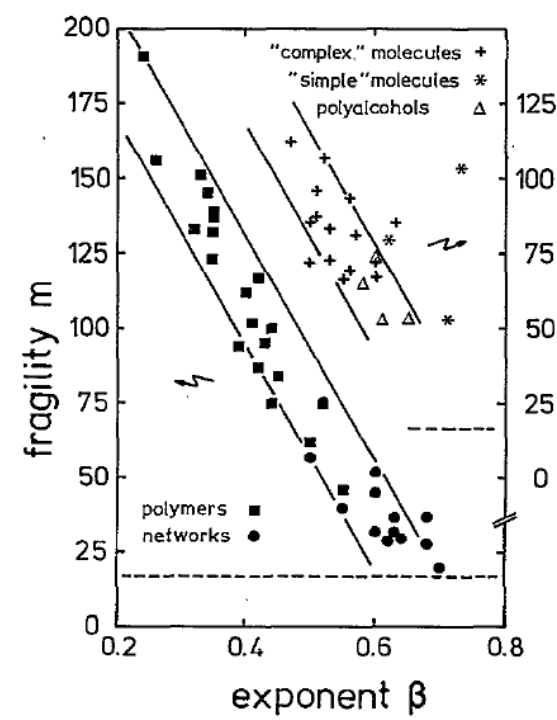

(a)

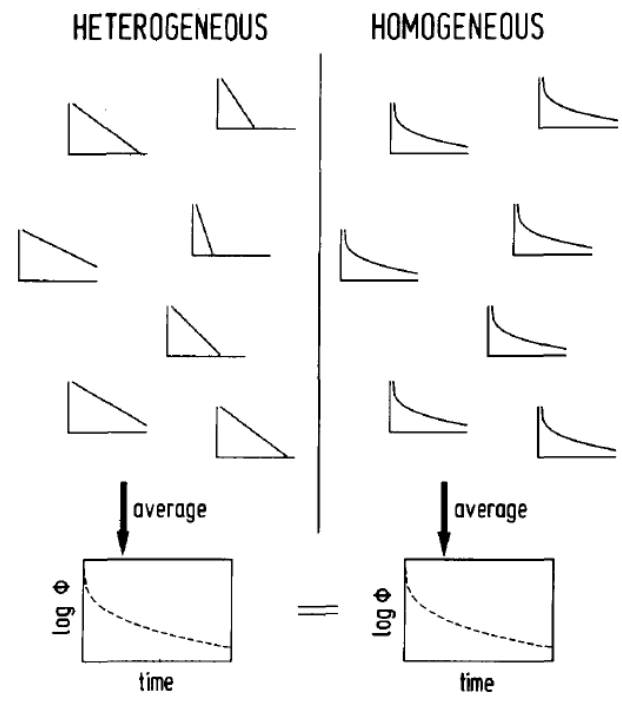

(b)

Figure 2.8 (a) Böhmer-plot of fragility $m$ against KWW-exponent $\beta_{K W W}$ for different classes of materials [14]. (b) Intrinsically exponential or non-exponential relaxations due to a heterogeneous or homogeneous explanation of the overall non-exponential form of the distribution of relaxations [111].

The depth of minima in the $\mathrm{PEL}$ is directly related to the necessary activation energy $E_{A}$ to overcome the barriers of these basins. If the thermal energy $k_{B} T$ is smaller than the necessary activation energy, the system can be described by a harmonic oscillation within a minimum, which is very well approximated by a parabolic. By a simple double-well potential [70] a hopping rate is defined. It describes the probability to leave a basin towards another minimum of notably different depth. If the thermal energy of the system is equal or larger than the activation energy. For jumps from one inherent state to another, the effective rate is just proportional to the inverse of the Arrhenius-Law in eq. 2.2. It is connected to a Debye-like decay [28, 29] with the relaxation time $\tau_{R}$ [90].

In the picture that is drawn by the model of the $\mathrm{PEL}$, the change from one metabasin to another should be described by the sum of the activation energies due to jumps through all the sub-basins, that lead from one metabasin to another. In contrast to this, the activation energy necessary for such a change of metabasins, which is equivalent to the process of primary relaxation, is larger, as it is described by the VFT.equation (cf. eq. 2.3). To characterize the relaxation function of primary relaxations, the Debye-like description has to be modified to a Kohlrausch-Williams-Watts (KWW) stretched exponential decay in time [69, 152], which is written as:

$$
g(t) \propto \exp \left[-\left(t / \tau_{K W W}\right)^{\beta_{K W W}}\right]
$$

The stretching exponent $\left.\beta_{K W W} \in\right] 0,1$ [ causes a non-exponential, stretched form of the function, which is more pronounced if $\beta_{K W W}$ is small. If this exponent is equal to one, eq. 2.9 corresponds to a symmetric Debye-relaxation with a single relaxation time equal to $\tau_{K W W}$. For many system $\beta_{K W W}$ is not constant with temperature even though this assumption is often made. It becomes smaller with decreasing temperature while approaching $T_{g}$ [36].

Often the KWW-function is used as a fitting function, e.g. to extract the relaxation timescale $\tau_{R}$. This timescale exhibits a VFT behavior if plotted against temperature for non-Arrhenius relaxations.

Glass-formers show a non-exponential distribution of relaxations for temperatures below the liquid state. Two extremes can describe this non-exponential form as displayed in fig. 2.8b. while it is reasonable to assume that most materials show a behavior resulting from a com- 
(a)

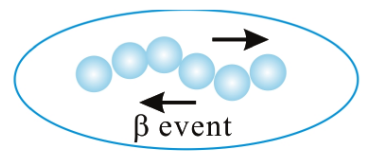

(b)

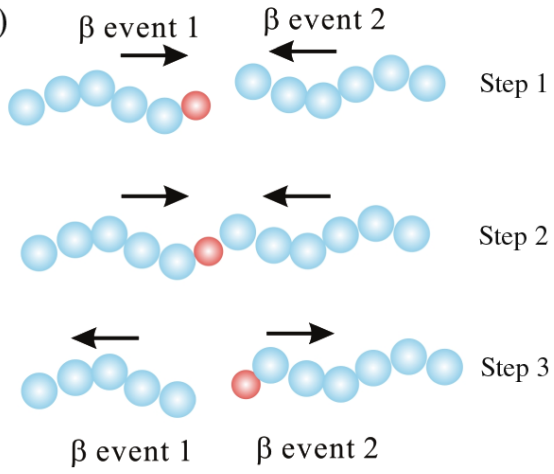

Figure 2.9 A schematic plot of the back and forth motion of string-like, cooperative $\beta$-processes (depicted in (a)) evolving in diffusion of single atoms (colored red in (b)) [159].

bination of both. On the one hand the non-exponential distribution can be explained by the contribution of solely intrinsically non-exponential modes. On the other hand, the very same overall non-exponential relaxation distribution can be gained by summing over exponential modes of different relaxation timescales $\tau_{i}$. One approach to find a combination of both cases is to define an index describing the grade of non-exponentiality of contributing modes by an intrinsic stretching exponent $\beta_{\text {in }}$ [13. Typically, supercooled liquids exhibit a heterogeneous distribution of exponential relaxations [35, 112]. Some plastic crystals are more precisely described by using an intrinsic stretching exponent which is empirically connected to a more homogeneous character of the distribution [153, 118].

As the exponent $\beta_{K W W}$ is connected to the fragility index $m$, as it decreases linearly with increasing $\beta_{K W W}$ within a material class according to the Böhmer plot [14], it exhibits the connection of fragility to the non-exponentiality of the relaxation distribution.

$$
m \approx(250 \pm 30)-320 \cdot \beta_{K W W}
$$

The larger the fragility index $m$ and the more fragile a system is, the smaller is the KWWexponent $\beta_{K W W}$ und thus the more non-exponential the distribution becomes. This relation holds at least within material classes, as this relation may have an offset in $\beta_{K W W}$ from one class to another (cf. fig. 2.8a).

\subsection{Microscopic Description of Rearrangements}

In 1965 Adam and Gibbs reported about the idea of cooperative rearranging regions (CRR). which they depicted as essential for relaxation processes in amorphous matter [1]. Many studies were performed to gain an understanding of the nature, volume, and impact of occurring CRR $\beta$, which are nowadays distinguished as $\alpha$ - and $\beta$-relaxations [62].

The $\beta$-process, also called secondary process, is observed dominantly at temperatures below $T_{g}$, and occurs on shorter timescales than primary relaxations. It merges with the $\alpha$-process at the merging temperature $T_{\text {merge }}>T_{g}$ (cf. fig. 2.6b). It was initially observed for polymer glasses [17], having its origin in rotational motions of side chains or functional groups of the molecules, also referred to as intra-molecular motions. Based on measurements on rigid polymers and molecular glasses showing a distinct signature of the $\beta$-process, Johari and Goldstein pointed out, that it has a more universal significance as it is also connected to inter-molecular motion [62]. This was confirmed by measurements on metallic glasses, which consist of atoms lacking internal degrees of freedom but still show a contribution to 
the secondary process [65]. Hence, inter-molecular motion is considered to be the process of fundamental importance, thus named Johari-Goldstein-relaxation [97].

Nuclear magnetic resonance experiments on simple glass formers show a very homogeneous nature of the $\beta$-processes due to small rotational motions of all dipoles in the system [144]. In contrast, molecular dynamics (MD) simulations [31, 139, 132] and numerical simulations [22] show rather heterogeneous behavior due to an approximately one-dimensional, string-like cooperative process. It is of a different nature than primary relaxations, and the cooperative regions consist of 10 to 100 atoms [124]. Experiments on colloidal glass formers confirm relaxation processes on the timescale of the $\beta$-process [146] and also mechanical measurements on metallic glasses report consistently [9]. In the $\mathrm{PEL} \beta$-relaxations are viewed as changes between inherent states, which are located within the same metabasin (cf. 2.3). In [52] it is shown that there is a direct correlation between $\beta$-processes and the elasticity of a sample. As the $\beta$-process is reversible due to the elastic matrix surrounding the string-like rearrangements the site holds a "memory" of the arrangement of particles before the secondary process occurred.

Moreover, the $\beta$-process shows a connection to the diffusion behavior in metallic glasses for the smallest constituting atoms [159]. Due to the back and forth motion of the string-like relaxations, atoms on the end of the strings may leave one string by following another, resulting in an effective displacement, i.e. diffusion of that atom (cf. fig. 2.9). Further details on secondary relaxation processes are given in the review by Yu et al. [160].

The $\alpha$-process becomes active within the glass transition range with increasing temperature, and is assumed as a spatio-temporal correlation between plastic events, which leads to irreversibility of the rearrangements [88]. Argon specified a plastic rearrangements of atomic regions of tens of atoms as a shear transformation zone (STZ) [5, 6, 129]. These STZ $\$$ are confined in an elastic matrix, which is described using Eshelby analysis [42, 41]. The Eshelby stress field is a quadrupolar elastic matrix displacement around the rearrangement's site. It can also be imagined as hypothetically cutting the very localized volume of the plastic rearrangement out of the matrix before it is sheared, then shear it so it takes another form, and inserting it back into its place. Now the matrix surrounding it has to compensate for the change in shape by elastic deformation. This elastic matrix can also be attributed for storage of energy, resulting in a memory of the original untransformed state. However, the restoring of the original state is very unlikely due to the relatively large number of involved particles. The Eshelby stress field can be used to describe the matrix around a plastic event, hitherto referred to as an STZ. The Eshelby field can also be applied to describe the surroundings of a string-like reversible $\beta$-relaxation. This fact emphasizes the similarity between single STZ $\beta$ and secondary processes. Both result in a typically elastic response of the surroundings, even though the former includes the occurrence of a very local plastic rearrangement and is thus to a certain extend of irreversible character [62], while the later is assumed to be fully elastic and thus of reversible nature. Primary relaxations involve rearrangements on a larger scale than secondary processes, and show a longer relaxation timescale (cf. fig. 2.7), which is connected to a higher degree of correlations between the constituents of a material. The cooperative shear model (CSM) combines the idea of STZ $\$$ with the PEL [64]. Here, primary relaxations correspond to a transition from one metabasin to another (cf. 2.3), and an $\alpha$-process is related to a succession of $\beta$-processes leading to this metabasin transition [52]. The metabasin transition comes along with a breakdown of elasticity, which might be understood as a (more or less complete) destruction of Eshelby stress fields surrounding sites of STZ $\$$. This comes along with the abundance of shape memory resulting in a plastic deformation of the material, which is irreversible. The CSM gives furthermore the basis for the calculation of the activation volume of an STZ [104] and thus the number of atoms involved in the plastic rearrangement. It is calculated to consist of roughly 690 atoms for $\mathrm{Pd}_{40} \mathrm{Ni}_{40} \mathrm{P}_{20}$ assuming a spherical geometry of the activation volume. In an experimental study on $\mathrm{Pd}_{77.5} \mathrm{Cu}_{6.0} \mathrm{Si}_{16.5}$ the strong correlation of the activation volume to temperature 
and applied stress is measured. It reveals an increase in activation volume of roughly a factor of 2 due to the influence of temperature and stress [127]. Other models were suggested to calculate length scales of correlated atoms in metallic glasses [120], e. g. by a connection of the width of the relaxation spectrum to the KWW-exponent $\beta_{K W W}$ (cf. eq. 2.9) by Moynihan [94, 95], or by the evaluation of higher harmonic contributions due to plastic events [16, 2 ].

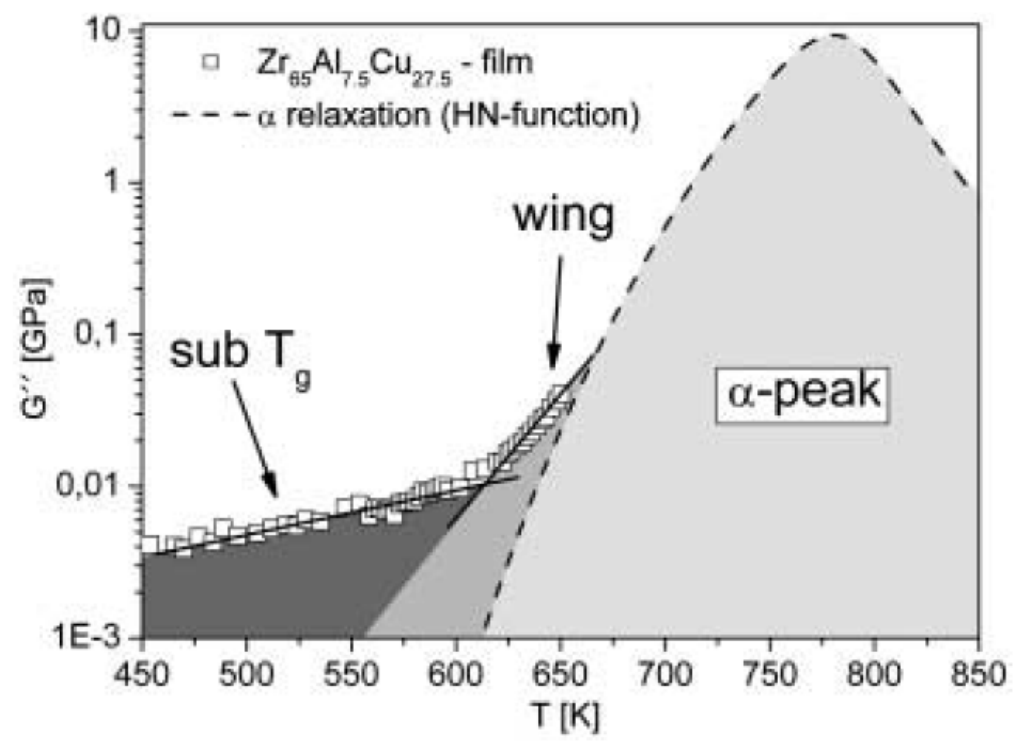

Figure 2.10 Plot of mechanical loss $G$ " versus temperature $T$ for glass-formers obtained by dynamical mechanical analysis at constant frequency [119].

In fig. 2.10 mechanical loss is plotted against temperature which shows striking similarity to fig. 2.7. This similarity is described by the principle of time-temperature-superposition, which is valid under quasi-equilibrium conditions, i.e. small excitations which probe the loss spectra [112]. At temperatures below $T_{g}$ the $\beta$-process becomes more and more activated with increasing temperature, superimposed with the $\alpha$-process which sets in at the glass transition temperature. This shows once more, that the occurrence of structural rearrangements depends highly on the kinetic energy of the system in the sense of temperature, but also on the timescale probing the system. The frequency spectrum can be shifted by measuring at higher temperature towards higher frequencies, as relaxation processes can follow on shorter timescales. The same argument can be applied to the temperature dependent plot in fig. 2.10. If the system were probed at a lower frequency, the loss spectrum was shifted to the left towards lower temperatures, as the onset of the glass transition depends on the probing timescale of the measurement.

The approach of distinguishable processes is clearly connected to a heterogeneous distribution of rearrangements. Experimentally this heterogeneity was measured by Samwer et al. [145], and a much broader distribution of local shear modulus is observed for metallic glasses than the case for crystalline materials.

According to Eshelby analysis, the strain field around a plastic inclusion or rearrangement is of quadrupolar nature [91]. This quadrupolar strain field is reflected in the local non-affine displacements of particles surrounding the plastic rearrangement (see fig. 2.11). Non-affine refers to that part of a particle's displacement which is not expected due to its elastic shear modulus [59]. As in metallic glasses and other amorphous bulk materials the displacement of atoms or particles is not an accessible quantity, molecular dynamics (MD) simulations, numerical analysis, and experiments on colloidal systems are used as tools to learn about the microstructure of relaxation processes. 

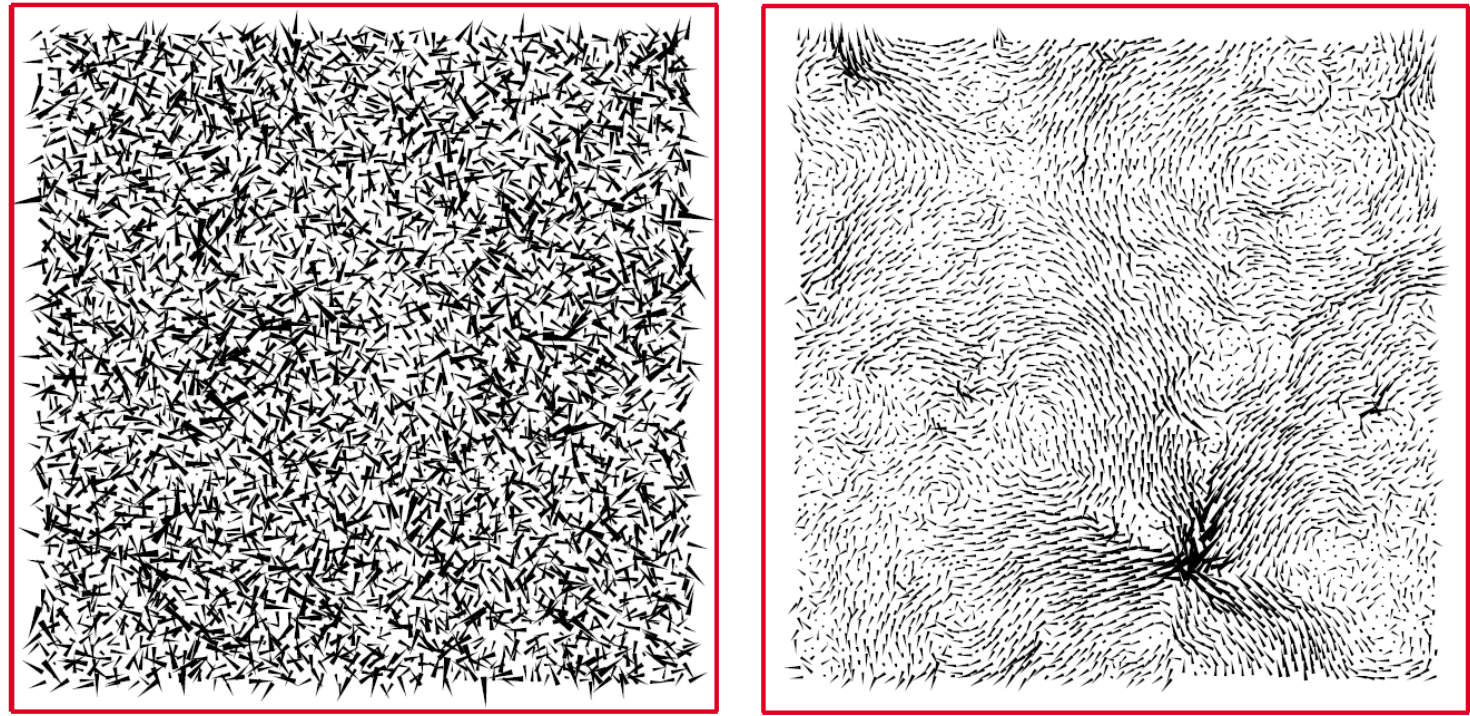

Figure 2.11 Force response to simple shear on the left and non-affine displacement field for the same state on the right based on numerical analysis [89].

By numerical simulations [89, 88, 137], an athermal system with a soft sphere potential is quasi-statically prepared under simple shear. Both the potential energy and the particle positions for each shear step are analyzed. Emphasized observations are upon others the quadrupolar nature of energy fluctuations and cascades of these during a single transition from one inherent state in the $\mathrm{PEL}$ to another. First of all, the observation of quadrupolar fluctuations in energy confirms the application of Eshelby analysis. Furthermore, the potential energy as a function of strain shows the existence of continuous segments, which are interrupted by sudden drops in stress and thus energy. This is a typical signature in systems under shear exhibiting plastic events as in fig. 2.12. For such a drop, many individual events become detectable under detailed analysis. This observation suits to the interpretation, that a drop in energy is connected with not only one event, but with a cascade of quadrupolar events. These cascades do not persist, but occur only during a single, infinitesimal small strain step of the simulation. They propagate through the system and vanish, and can thus be regarded as of a rather fluctuating nature (cf. fig. 2.13). If the system is driven more intensely, concurrently occurring events are to be expected in space such that quadrupolar patterns of several events superimpose. The cascades of $\overline{S T Z} \mathbf{s}$ lead to the breakdown of elasticity. What remains unclear in comparison to the interpretation of the model on the $\mathrm{PEL}$ is, why these cascades occur during transitions from one inherent state to another in case of the reported simulation.

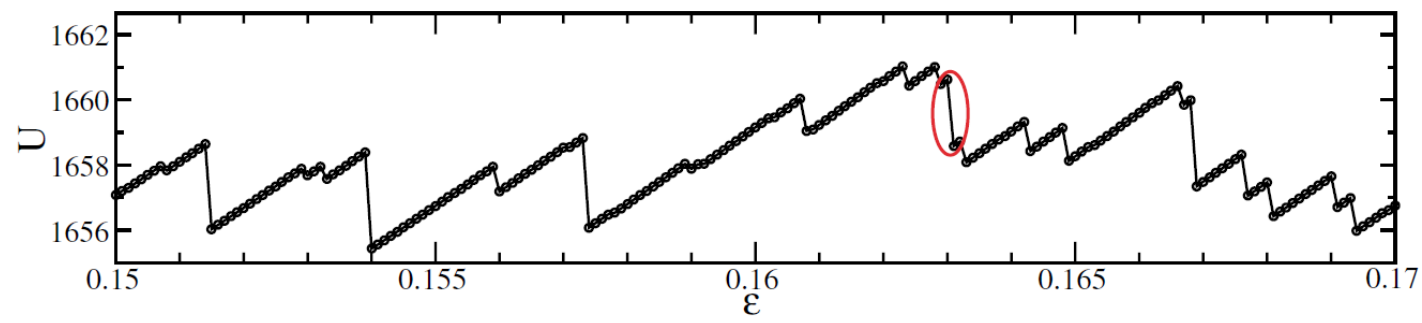

Figure 2.12 Potential energy $U$ as a function of strain $\epsilon$ under quasi-static shear based on numerical analysis [88]. 

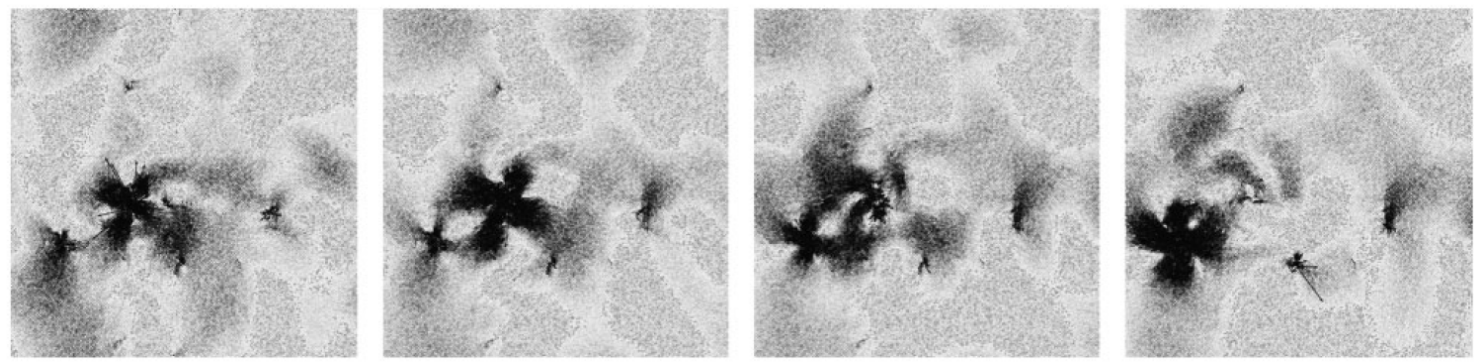

Figure 2.13 Instantaneous velocity field of a growing shear transformation zone, triggering the flip of another zone in its vicinity based on MD simulations [82].

The coupling model by Ngai [100, 98, 99] emphasizes the strong correlation of $\alpha$ - and $\beta$ relaxation very clearly. It connects the rather independent nature of secondary relaxations to primitive $\alpha$-relaxations exhibiting a similarly independent relaxation behavior. The timescale of this primitive $\alpha$-relaxation $\tau_{0}$ can be extracted by the definition of an onset of slowing down the independent relaxations at the time $t_{c}$. The magnitude of $t_{c}$ is dependent on the strength of interaction between relaxations. Before $t_{c}\left(t<t_{c}\right)$ the response of the $\alpha$-relaxation is assumed to be purely exponential as the case for $\beta$-relaxations (cf. eq. 2.2). After $t_{c}\left(t>t_{c}\right)$ the cooperative relaxation occurs as $\tau_{\alpha}$ as in the stretched-exponential KWW function (eq. 2.9), whose exponent is directly connected to the coupling parameter $n$, and which is typically observed in case of $\alpha$-relaxation (cf. eq. 2.3). These timescales can be connected to another by assuming continuity of the exponential and stretched exponential behavior at $t=t_{c}$ according to:

$$
\tau_{\alpha}=\left(t_{c}^{-n} \tau_{0}\right)^{1 /(1-n)}
$$

As a result of these assumptions, the timescale of secondary relaxation is at the glass transition temperature of comparable order of magnitude as the timescale of primitive $\alpha$-relaxations $\left(\tau_{\beta}\left(T_{g}\right) \approx \tau_{0}\left(T_{g}\right)\right)$. This indicates a close relation of $\alpha$ - and $\beta$-relaxations, even though the primitive $\alpha$-relaxation and the $\beta$-relaxation is not assumed to be identical.

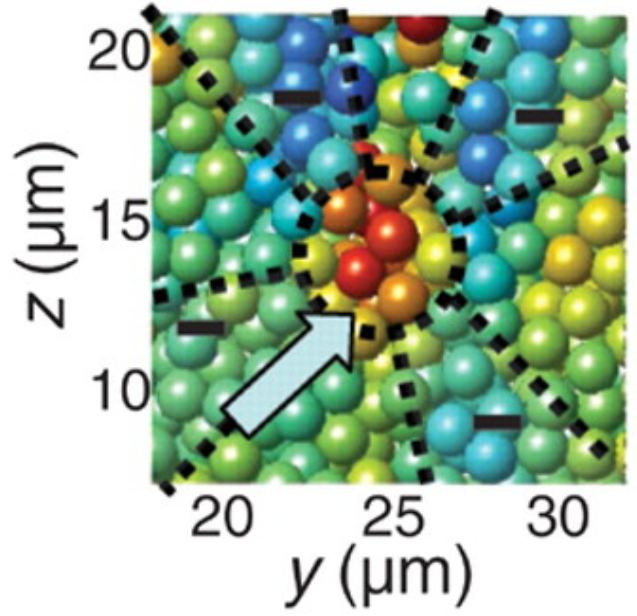

(a)

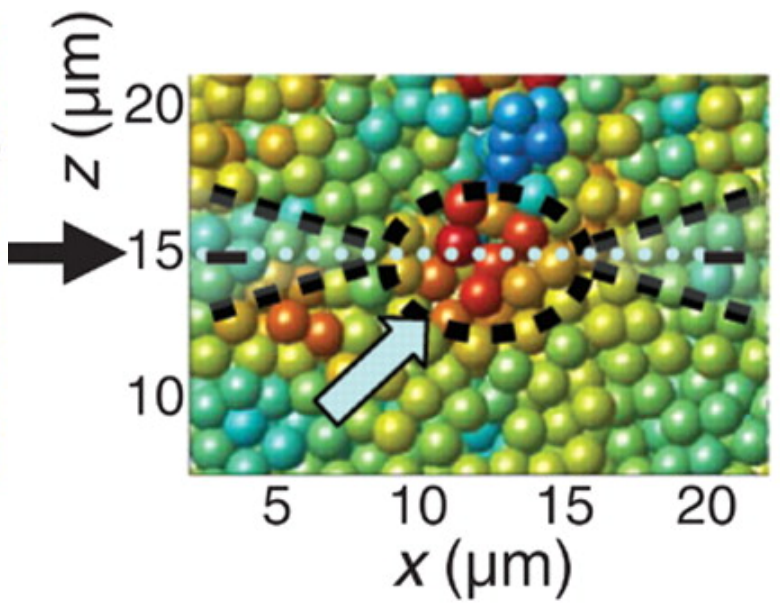

(b)

Figure 2.14 Spatial distribution of strain (a) in y-z- and (b) x-z-plane for an STZ, with particles colored corresponding to their shear strain (cf. colorscale in fig. 2.15) based on experiments on colloidal systems [122].

In colloidal systems, sites of structural rearrangements are identified, and the quadrupolar distribution of displacements surrounding an STZ is measured in three dimensions [122, 20, 19]. STZ $\$$ are induced both thermally, appearing as thermal strain fluctuations, and mechanically 
by a shear stress close to the yielding stress of the system (cf. section 2.6). Fig. 2.14 shows the response of a glass to a shear strain applied in y-direction, in (a) a section of the $y$-zplane of the system is shown. The arrow points towards the center of an STZ, consisting of particles exhibiting a high positive strain and thus a high positive displacement (red colored). Surrounding this plastic core, four regions of negative shear (indicated by blue regions) are located, which correspond to the typical form of the quadruploar displacement field due to a structural rearrangement in its center [41]. The observed displacements are constant within the region of the core, which extends over a radius of $r_{c} \propto 3.07 r_{p}$, where $r_{p}$ denotes the average particle radius of the system. For radial distances larger than $r_{c}$ the displacement field decays with $\propto r^{-3}$, as expected [41]. Fig. 2.14b shows a section of the colloidal system in the $x$-z-plane for the same STZ as in fig. 2.14a. The core seems to be slightly elongated with a width $w_{c} \propto 6.67 r_{p}$. Moreover, along the $x$-direction the core is enclosed by regions of negative strain.

Not only single STZ $\$$ are observed in the colloidal system, but also a coupling between persisting and initialized STZ $\$$ in their vicinity, such that the nucleation of new shear transformation zones is faciliated. Branches of positive strain, i.e. positive displacements, evolve between STZs with increasing experimental time, as shown in fig. 2.15. Red colored regions correspond to STZ $\beta$, yellow colored particles indicate regions of enhanced strain. Clearly, STZ $\beta$ connect to a kind of network which intermingles and finally permeates the entire plane. Subfigures $A$ to $C$ in fig. 2.15 show the spatial distribution of strain of all particles in the plane, while subfigures $D$ to $F$ are restricted to enhanced strain values.
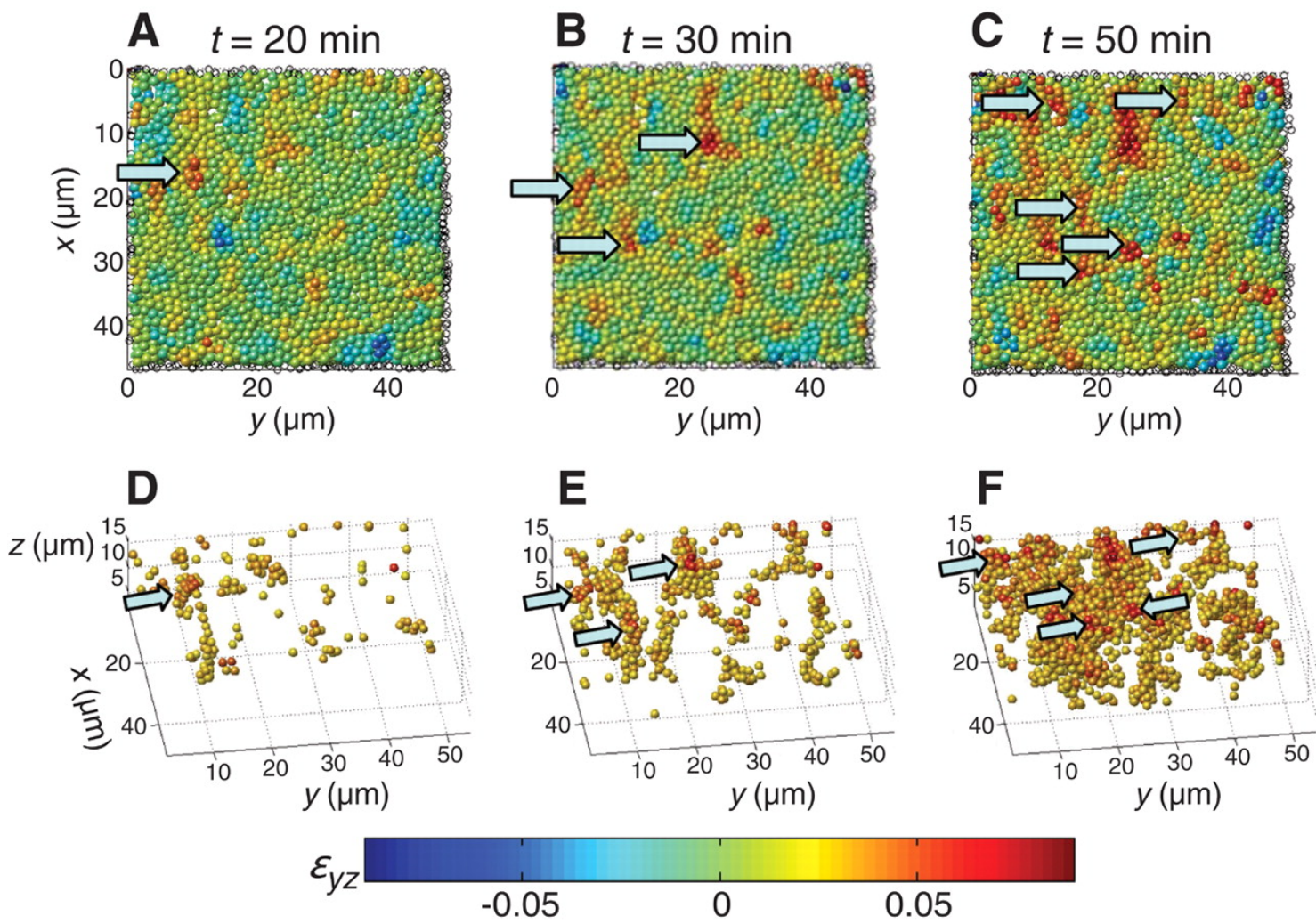

Figure 2.15 Evolution of strain with experimental time after $20 \mathrm{~min}(\mathbf{A}), 30 \mathrm{~min}$ (B), and $50 \mathrm{~min}$ (C) with arrows indicating STZ for $x$-y-frames of $5 \mu \mathrm{m}$ thickness in z-direction. $\mathbf{D}$ to $\mathbf{F}$ for same experimental times, but including highly strained particles for $x$-y-frames of $16 \mu \mathrm{m}$ thickness in $z-$ direction, based on experiments on colloidal systems [122].

A further topic highly related to the microstructure of rearrangements is the formation of shear bands, which evolve characteristically in a system driven by very high stress or strain. In metallic glasses shear bands inherit a two-dimensional topology of a highly strained core of 
a few $\mathrm{nm}$ in thickness, surrounded by a tens of $\mu \mathrm{m}$ thick soft zone [87]. Shear bands become system spanning and cause the failure of the system and are of permanent character. An important question is if and how cascades of STZ $\$$, which are of a fluctuating, transient nature, correlate to form persistent shear bands. The evolution of shear bands from STZ $\$$ is assumed to be the underlying mechanism, which occurs when a critical density of STZs is achieved by mechanical excitation [49]. In contradiction, Le Bouil et al. [80] published experimental data based on a granular material under stress, showing distinct differences between the nature of a cooperative avalanche network as observed in [122], too, and a persistent shear band. The angle $\theta$ of the observed microstructural rearrangements relative to the direction of applied shear differs significantly by $20^{\circ}$ (transient avalanche network with $\theta \approx \pm 45^{\circ}$; permanent shear band with $\theta \approx \pm 65^{\circ}$ ). Moreover the avalanche network coexists near failure with the permanent shear band, and the authors claim that the relationship between these transient microbands [i.e. avalanches] and the final permanent frictional shear band is more complex than the description of a final persistent shear-band formation as a mere growing cascade of local rearrangements. However, an alternative model to describe the initiation of shear bands is not given, thus the emergence of persistent shear-bands from cascades of local rearrangements is currently considered as the most appropriate description.

\subsection{Influence of Mechanical Excitation on Structural Rearrange- ments}

As described in the section on the microstructure of relaxation processes (sec. 2.5), viscosity does not only depend on the material's temperature, but also on its structure [73, 76]. It is determined by the history of the glass, e.g. the preparation technique, but is also depending on how it is exposed to mechanical excitation [108].

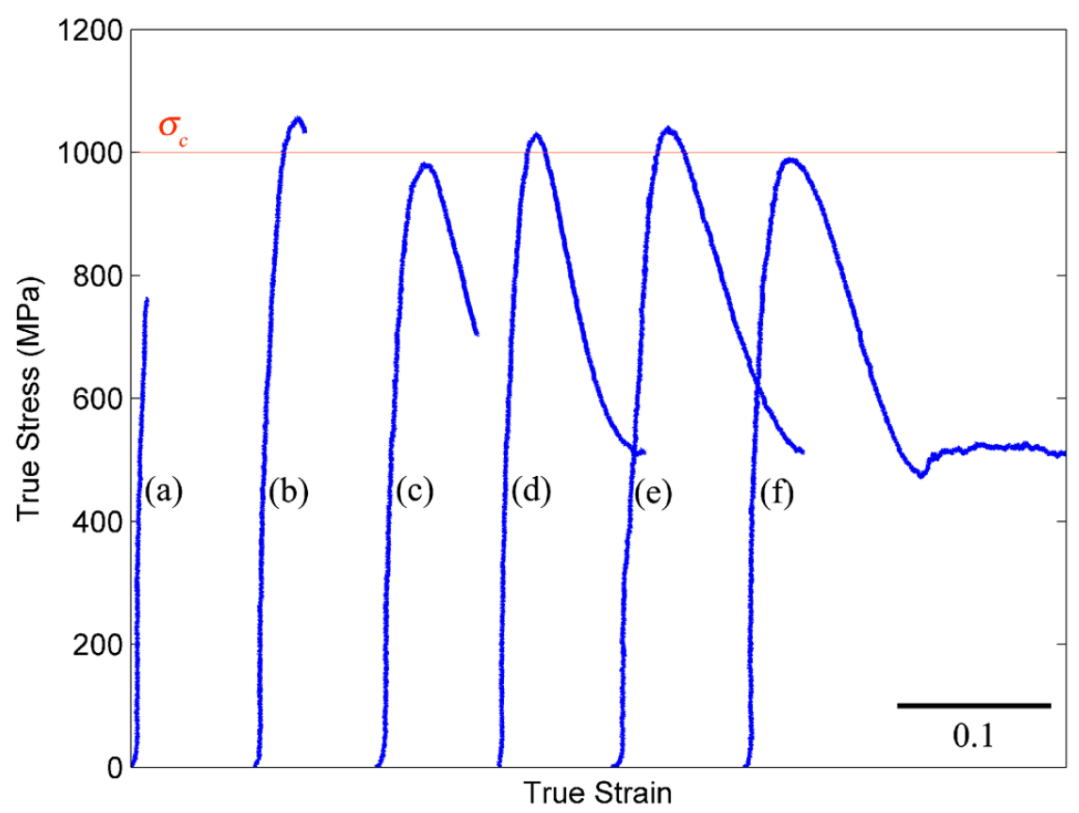

Figure 2.16 Stress-strain-curves measured under compressive mechanical load up to six different total strains on a metallic glass [52].

The response in stress due to an applied strain is plotted for six different total strains in fig. 2.16. It is measured during mechanical compression of amorphous metallic glass samples made of $\mathrm{Pd}_{43} \mathrm{Ni}_{10} \mathrm{Cu}_{27} \mathrm{P}_{20}$ at a constant strain rate of $10^{-4}$ at a temperatures of $548 \mathrm{~K}$, which is $21 \mathrm{~K}$ below the calorimetric glass transition temperature [52]. In the stress-strain- 
curves, three distinctive regimes can be accounted for, which are due to different mechanism of rearrangement within the material.

The first stress-strain-curve in fig. 2.16 marked as (a) shows a linear-like increase which is declared as the elastic part of the stress-strain-curve. In the case of an compression or shear experiment, its slope is defined as the shear modulus $G$ :

$$
\sigma=G \cdot \varepsilon
$$

It gives the relation between stress $\sigma$ and strain $\epsilon$ for low values, where the material's response is assumed to be purely elastic, i.e. where the occurrence of plastic processes is small. The elastic regime ranges from lowest values in stress and strain up to the critical stress $\sigma_{c}$, at which the stress-strain-curve has its maximum (cf. (b)), which is referred to as the yield point. In the model of the $\mathrm{PEL}$ (cf. ch. 2.3), this first regime of the stress-strain-curve is dominated by intrabasin hopping correlated to $\beta$-processes or single STZ $\beta$ [52]. For stresses $\sigma$ larger than the critical stress, changes between metabasins and thus irreversible processes become more and more probable. In curves (c), (d), and (e) the stress-strain-curve shows a decreasing behavior, which is due to an increasing tendency towards irreversible processes. Thus, cascades or avalanches of STZ $\$$ are expected which are connected to energy dissipation. This anelastic regime ends where the stress-strain-curve saturates to a plateau as in (f). The plateau regime of constant stress even though increasing strain is called the plastic regime. Here, the interbasin hopping rate, correlated to stress drops, and the elastic increase in stress balances to a steady state. Here, shear band formation is typically observed, as described microstructurally in section 2.5 .

In [61] an order parameter is introduced, which compares the different configurations of the system while being strained. This parameter describes the overlap between the initial configuration at zero strain with any configuration that occurs during the straining of the system. In the regime of the yield point, this order parameter can be used to identify a phase transition from the quasi-elastic to the elasto-plastic state. Here, all occurring configurations lose their overlap with the initial configuration, while at slightly lower strain the overlap of configurations is still given. The characteristics of configurations in the two phases are regarded the same, but the number of available configurations increases vastly at the yield point, when the proclaimed phase transition occurs.

Whether shear band formation occurs, i.e. inhomogeneous flow is established, or if the material deforms homogeneously, depends on several parameters. These are the temperature $T$ relative to the glass transition temperature $T_{g}$, the applied shear stress relative to the shear modulus (in fig. 2.17 depicted as $\tau$ and $\mu$, respectively), and the strain rate $\dot{\varepsilon}$, as depicted in the mechanical deformation maps as introduced by Spaepen [130]. In fig. 2.17 for relative stresses below a threshold of roughly 0.02 the system deforms either elastically for temperatures below the glass transition temperature or due to homogeneous flow. Depending on the strain rate and accordingly the stress the material behaves liquid-like, i.e. Newtonian as for lower stresses, or solid-like, i.e. non-Newtonian as for higher stress. If the normalized stress exceeds the threshold of 0.02 , the material exhibits inhomogeneous deformation as described by the cooperative shear model [64], connected to shear banding and thus very localized strain (cf. sec. 2.5).

If $\Delta x$ describes the relative displacement between two parallel plates with the space in between filled by a liquid-like material, and $d$ is the distance between the plates, the shear strain $\varepsilon$ mentioned above is defined by $\varepsilon=\Delta x / d$ [78]. With the stress $\sigma=F / A$ involving force $F$ and area of a plate $A$, and $\dot{\varepsilon}$ as the derivative of shear strain, the viscosity is defined as $\sigma=\eta \cdot \dot{\varepsilon}$. Maxwell [90] connected strain rate $\dot{\varepsilon}$, viscosity $\eta$, and shear modulus $G$ as

$$
\dot{\varepsilon}=\frac{\sigma}{\eta}+\frac{\dot{\sigma}}{G}
$$




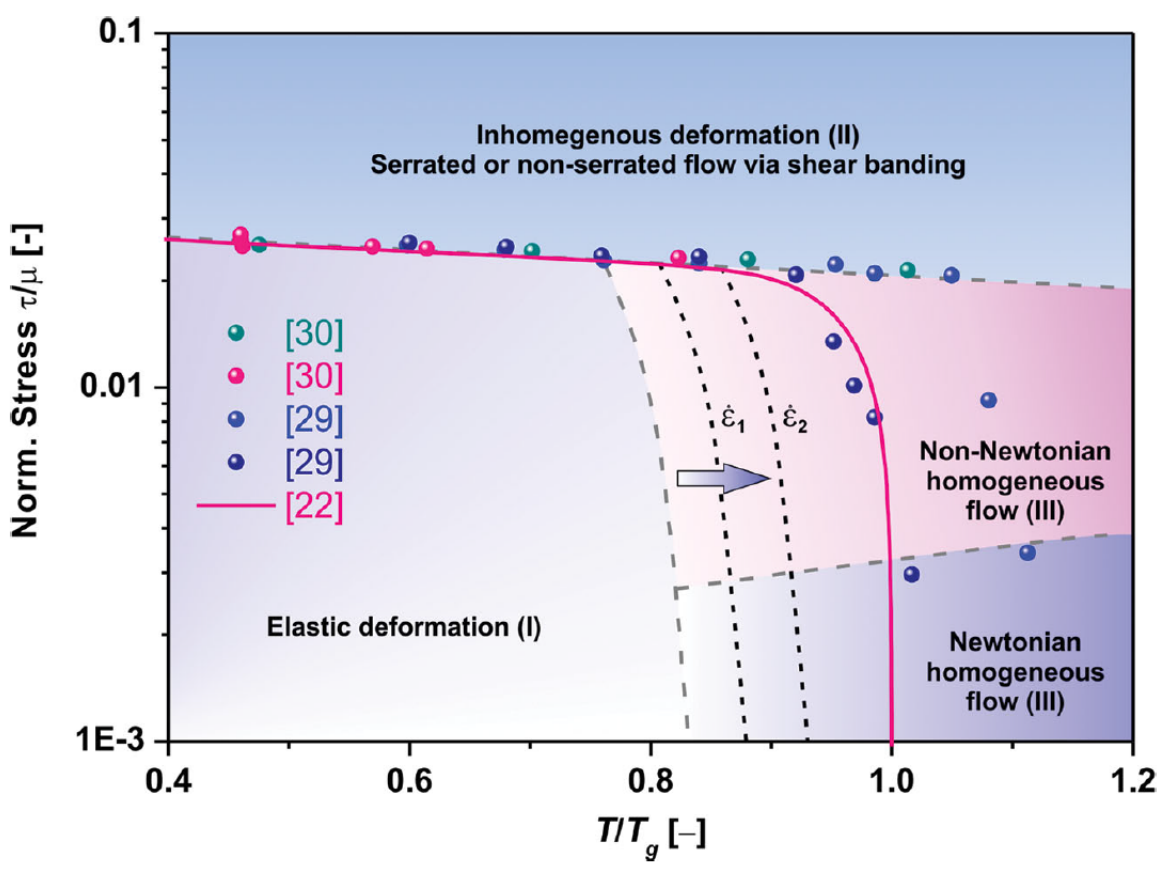

Figure 2.17 Mechanical deformation map depicting deformation mechanisms in metallic glasses depending on normalized temperature $T / T_{g}$, normalized stress $\tau / \mu$, and strain rate $\dot{\epsilon}$. [86].

applying for both the steady-state flow in liquids $(\dot{\sigma}=0)$, also called Newtonian flow, and for solids $(\eta=\infty)$, which is non-Newtonian. The broad range in between is a coupling of both extremes called visco-elastic behavior. If the Newtonian part was represented by a spring and the non-Newtonian part by a dash-pot, the Maxwell-model would couple both in series. Another model, called Kelvin-Voigt-model, connects both extremes in a parallel manner [45, 21] and thus attributes to the creep behavior typical for visco-elastic materials. If one assumes an abrupt shearing displacement $\dot{\varepsilon}(t)=\varepsilon_{0} \delta(t)$ within the Maxwell-model, integration yields for $t=0$ :

$$
\varepsilon_{0}=\frac{\sigma}{G}
$$

It becomes obvious that the shear modulus $G$ is instantaneous or frequency-independent and thus it is often denoted as $G_{\infty}$.

For $t>0$ the stress decays exponentially, thus the shear modulus can be connected to a relaxation timescale $\tau$ :

$$
\tau=\frac{\eta}{G_{\infty}}
$$

These equations reflect again the complexity of the glass transition, as the material between the fictive plates behaves solid-like on timescale much shorter than $\tau$, as well as for large shear rates, but behaves liquid-like on timescales which are comparatively long towards $\tau$ [34.

In case of a tensile experiment the slope of the elastic regime of a stress-strain-curve, which is described by Hook's Law, is defined as the Young's modulus $E$ [92].

$$
\sigma=E \cdot \varepsilon
$$

Shear modulus $G$ and Young's modulus $E$ are related by the Poisson's ration $\nu$ via [107, 154]:

$$
E=2 G(1+\nu)
$$

The Poisson's ratio depends on the material, e.g. for the metallic glass $\mathrm{Pd}_{40} \mathrm{Ni}_{40} \mathrm{P}_{20}$ the value is determined to 0.403 [102]. 
The cooperative shear model (CSM) relates the value of the shear modulus $G$ to the curvature of the potential energy density $\phi$ as a function of strain $\varepsilon$ by

$$
G=\phi^{\prime \prime}=\frac{d^{2} \phi}{d \varepsilon^{2}} .
$$

This gives a connection of the shear modulus $G$ with the position of the accounted system regarding its depth in the $\mathrm{PEL}$ within a basin [161, 52, 63]. For lower states of potential energy of the system the curvature of the basin, which the system inherits, becomes larger. Thus, the value for the shear modulus exhibits higher values, if the density of minima in the $\mathrm{PEL}$ can be assumed as constant. Under strain, the curvature of these minima is reduced, resulting in a decrease in shear modulus in the picture of the PEL.

\subsection{Response to High Field Excitations}

MD-simulations and experiments on colloidal systems show that mechanical excitation is an important tool to probe structural relaxations in form of microstructural rearrangements (see section 2.5). Indeed, stress and strain play an important role, somehow similar to temperature itself, regarding the influence on the onset of the glass transition region. As has been shown in [11] and [50] by numerical analysis and simulations of Zr-based metallic glass, the glass transition temperature $T_{g}$ is directly linked to mechanical yielding.

The influence of applied stress and temperature on the damping behavior of a Pd-based metallic glass is measured experimentally for temperatures below $T_{g}$ by Schwabe et al. [126]. In fig. 2.18 the timescale of the damping during a creep measurement, denoted as delay-time, is plotted and clearly increases with increasing stress and temperature. For small temperatures and stresses the system is interpreted as elastically responding, connected to a small degree of dissipated energy and minor damping. With increasing stress and temperature, more and more energy is dissipated in the system, connected to the activation of irreversible plastic processes connected to enhanced damping. The fact that stress and temperature influence the damping behavior in a similar fashion leads to the conclusion, that similar processes of structural rearrangements are triggered by both parameters.

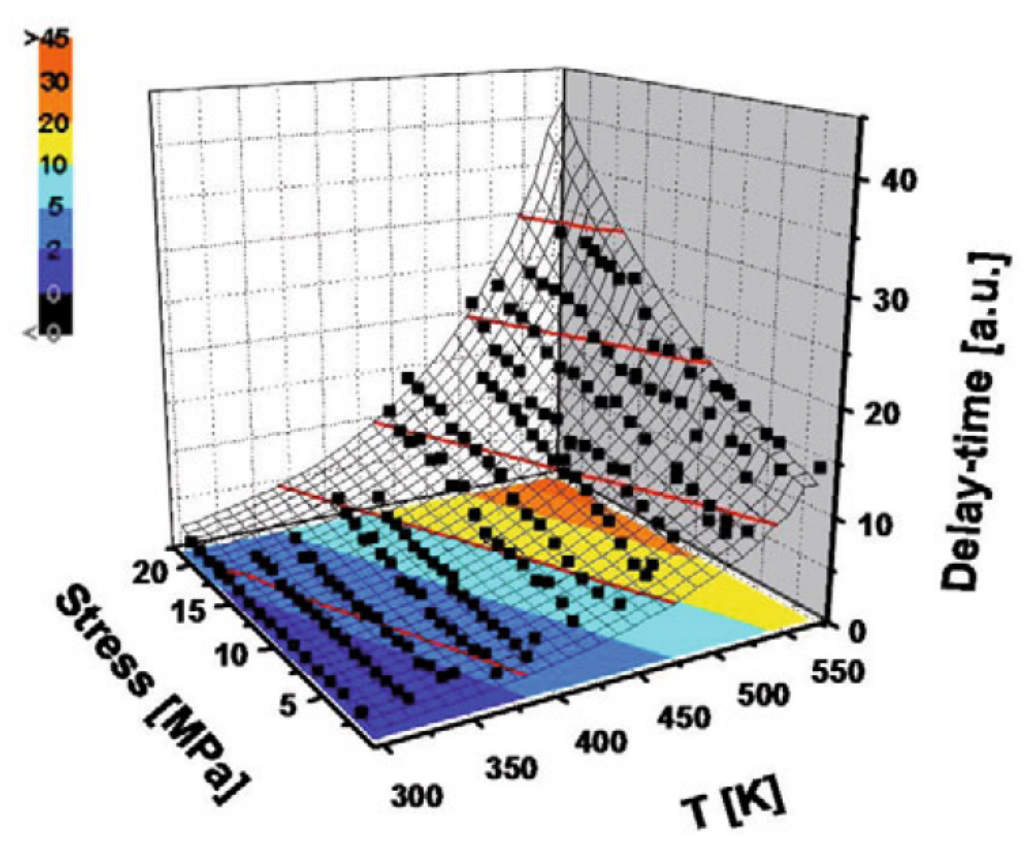

Figure 2.18 Influence of temperature $T$ and stress on the damping controlled delay-time of $\mathrm{Pd}_{77.5} \mathrm{Cu}_{6.0} \mathrm{Si}_{16.5}$ determined by mechanical creep measurements [126]. 


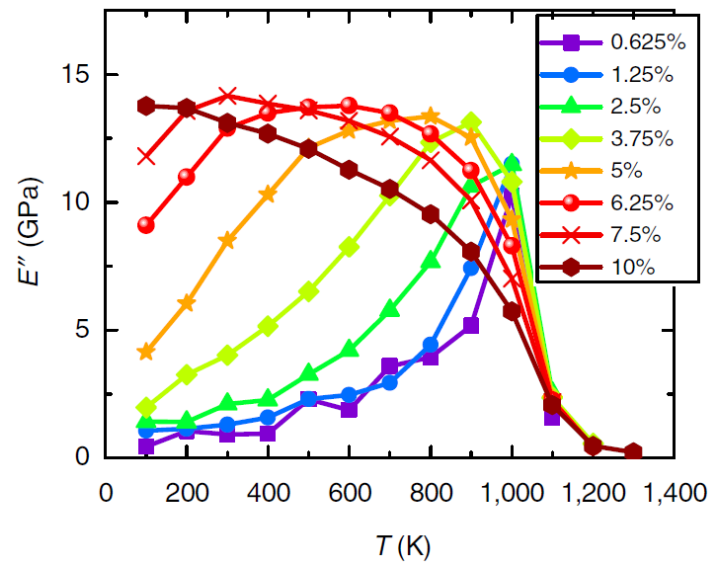

(a)

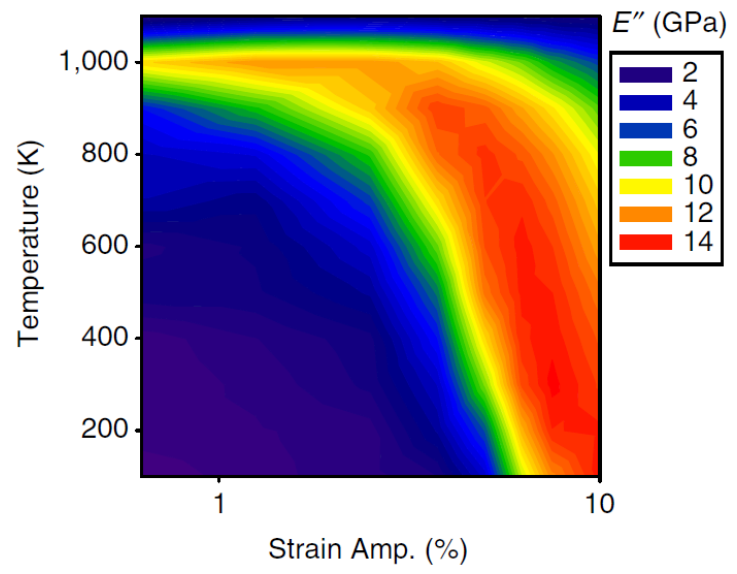

(b)

Figure 2.19 (a) Mechanical loss $E^{\prime \prime}$ as a function of temperature $T$, and (b) mechanical loss $E^{\prime \prime}$ as a function of temperature $T$ and strain amplitude $\varepsilon_{A}$, both based on MD-simulations [157].

Yu et al. [157] connected the mechanically driven glass transition with a change in fragility $m$ by performing MD-simulation of dynamical mechanical spectroscopy on a model metallic glass. As in fig. 2.19, with increasing strain the spectrum of the mechanical loss spectrum broadens significantly in a nonlinear fashion, the low-temperature flank is dominantly affected. A fragile-to-strong transition is occurring with increasing strain amplitude as indicated by the connection of the increasing width of the loss spectrum to the reduction of fragility [27]. The calculation of the fragility via eq. 2.4 using the dependence of relaxation timescales of the $\alpha$-process on inverse temperature confirms this observation. The relative change in fragility is plotted in fig. 2.20 .

This result suits the influence of stress on the $\mathrm{PEL}$ as the landscape is tilted with increasing stress as in fig. 2.21. The tilting leads to the destruction of inherent states in the PEL by the applied strain, as the inherent state collides with a transient state. Furthermore, the system drops in energy in an irreversible way, as the obtained state in the $\mathrm{PEL}$ before the destruction of the occupied inherent state is not existent anymore. Schematically, an applied stress of significant amplitude leads to a PEL, which consists of few deep minima, but rather homogeneous barriers, as depicted in fig. 2.5. which corresponds qualitatively to the landscape typical for a rather strong glass-former (cf. section 2.3).

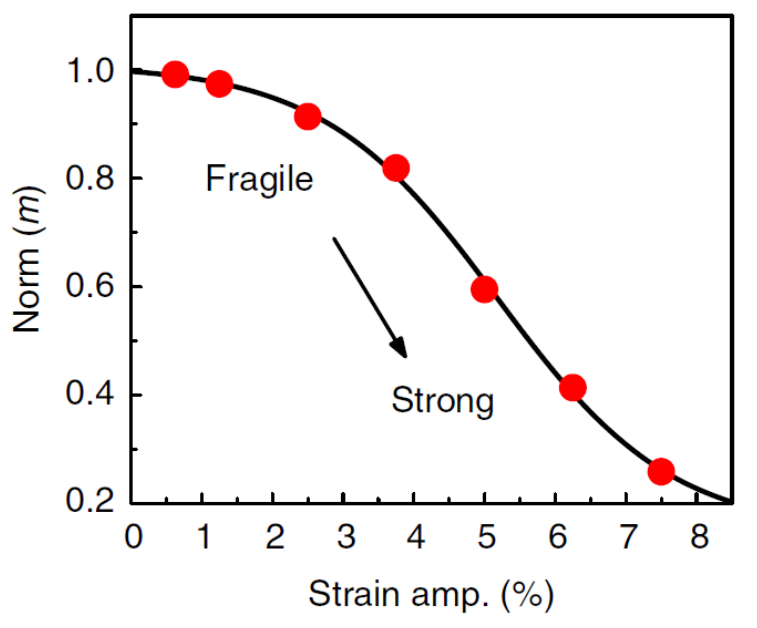

Figure 2.20 Normalized fragility index $\operatorname{Norm}(m)=m_{\text {measured }} / m_{\varepsilon_{A}, \text { min }}$ as a function of strain amplitude $\varepsilon_{A}$ deduced from MD-simulations [157]. 


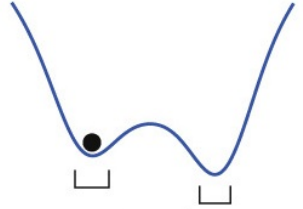

Basins

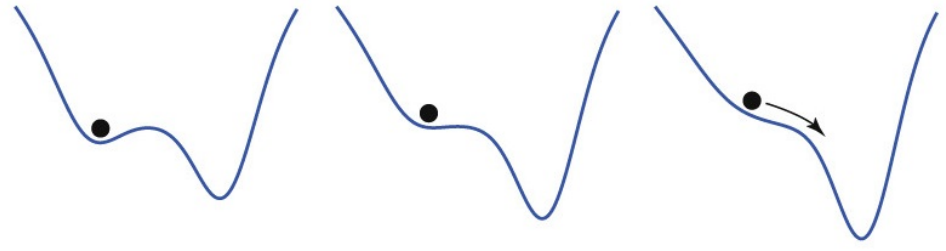

Increasing shear stress

Figure 2.21 Scheme of the change of the potential energy landscape due to applied shear stress [59].

Interestingly, both the temperature-induced and the strain driven glass transition in metallic glasses are attributed to the Lindemann criterion [83]. It is a theory for the melting of a crystalline solid, considering the range of displacements of atoms as an indicator for the phase transition. With increasing temperature the amplitude of the vibrational displacements of particles increases. By defining a critical value, the vibrational amplitude at which the material is considered as melted is identified. The threshold is depending on the interaction potential and is in most cases between 0.1 and 0.2. As an universal observation, the $\alpha$-relaxation in metallic glasses occurs if the most probable atomic displacement exceeds the critical value. This is $20 \%$ of the average atomic displacement and reflects the position of the inflection point in the effective inter-atomic potential. This approach is independent of the driving parameter for the transition, which can be temperature, stress, or others [158].

\subsubsection{Nonlinear Response in Dielectric Spectroscopy}

The method of MD-dynamical mechanical spectroscopy used by Yu et al. [157] has its example in dielectric spectroscopy, which is used to experimentally probe the nonlinear response of amorphous materials to high field excitation and deduce information on the heterogeneity of the relaxation spectrum [117].

Linear behavior exhibits a proportionality between excitation and response signal, which are the electric field $E$ and the polarization $P$ in case of dielectric experiments, depicted as a dashed line in fig. 2.22. Under high field excitation the sample response exhibits a nonlinear relation between polarization and electric field, while the onset of nonlinearity depends on the resolution limit of the measurement technique [113]. Two different approaches can be chosen to enter the nonlinear regime. Either the application of a large amplitude ac field $E_{a c}$ as discussed below, or a small ac field superimposed on a large dc field $E_{d c+a c}$.

Typically, nonlinearity in dielectric response experiments is described by

$$
\frac{P(t)}{\varepsilon_{0}}=\chi E(t)+\chi^{(3)} E^{3}(t)+\chi^{(5)} E^{5}(t)+\chi^{(7)} E^{7}(t) .
$$

Under the assumption that contributions higher than the third order result in negligible output, the frequency-dependent susceptibilities $\chi^{(n)}(\omega)$ can be expressed as [138]:

$$
\begin{gathered}
\frac{P_{1}(\omega)}{\varepsilon_{0} E_{0}}=\chi_{1}(\omega)+\frac{3 E_{0}^{2}}{4} \chi_{1}^{(3)}(\omega)=\chi_{1}(\omega)\left(1+\Delta \ln \chi_{1}(\omega)\right) \\
\frac{P_{3}(3 \omega)}{\varepsilon_{0} E_{0}}=\frac{E_{0}^{2}}{4} \chi_{3}^{(3)}(3 \omega)
\end{gathered}
$$

The indices of $\chi_{k}^{(n)}$ denote the power $n$ of the electric field associated with the susceptibility and the Fourier component $k$ of the polarization. Evidently, the nonlinear response results in two effects, which are the change of the response in the fundamental frequency $\omega$, and the generation of higher harmonic contributions. 


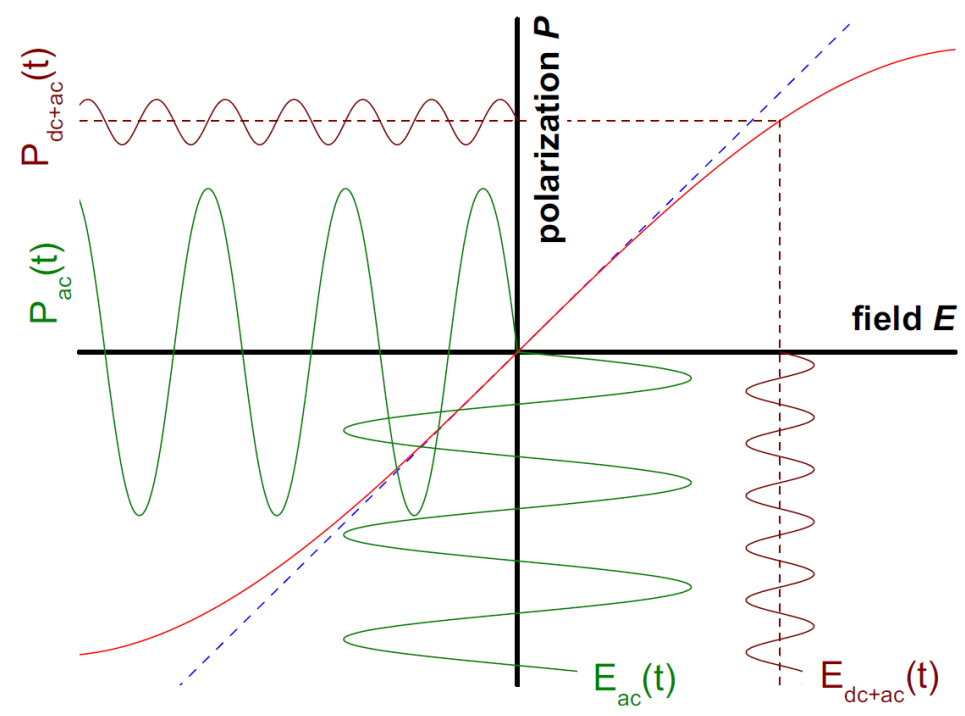

Figure 2.22 Scheme of nonlinear response as a third-order polynomial (solid line) deviating from the linear behavior (dashed line) for an exciting electric field $E$ and polarization response $P$ [113].

To approach the nonlinear response regime a sinusoidal voltage, in other means an electric field $E_{x}(t)=E_{0} \sin (\omega t)$, is applied to a dielectric sample at a certain angular frequency $\omega$. The field amplitude $E_{0}$ is switched from a typically low value to a high value, and back to the initial low value, each for a fixed number of cycles. The cycling is chosen to be sufficiently long to measure the time-dependent response, but short enough to avoid Joule heating effects [147]. Voltage and resulting current are measured, analyzed using period-byperiod Fourier analysis to extract amplitude and phase difference $\Delta \phi$ at desired frequencies $\omega, 2 \omega, 3 \omega$, or higher harmonic orders. The storage and loss dielectric susceptibility $\varepsilon^{\prime}(\omega)$ and $\varepsilon^{\prime \prime}(\omega)$, as well as the loss $\tan \delta=\tan (\pi / 2-\Delta \phi)$ are analyzed. This is performed for several frequencies $\nu=\omega(2 \pi)^{-1}$ in a range covering several orders of magnitude to measure the frequency- and time-dependent nonlinear response signal. The resulting data in dielectric loss for low-field and high-field amplitudes are plotted in fig. 2.23 for glycerol.

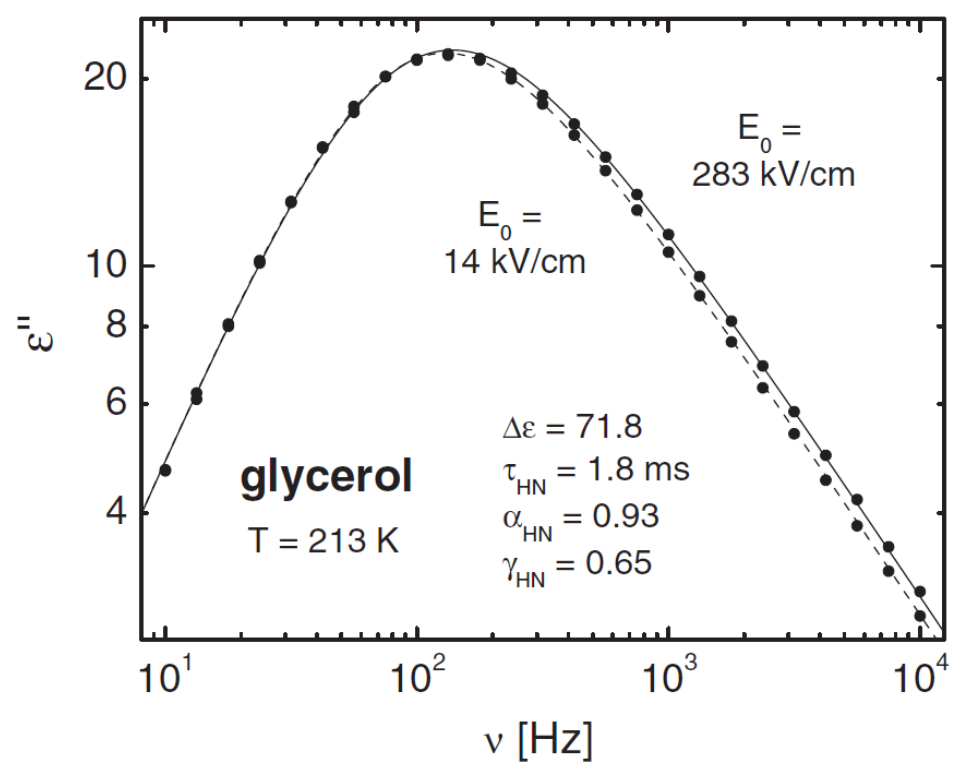

Figure 2.23 Dielectric loss $\varepsilon^{\prime \prime}(\nu)$ (symbols) against frequency $\nu$ for glycerol at $T=213 \mathrm{~K}$ at a low-field amplitude of $E_{0}=14 \mathrm{kV} \mathrm{cm}^{-1}$ (lower data) and a high-field amplitude of $E_{0}=283 \mathrm{kV} \mathrm{cm}^{-1}$ (upper data). The line is based on assumptions due to the box-model [148]. 
On the high-frequency flank of the spectrum, the high-field loss data shows clearly values, which are shifted towards higher frequencies, with the shifting effect well captured by the so called box-model [117]. It aims on describing the nonlinear effects occurring due to energy absorption from a high time-dependent external field under following assumptions. The overall non-exponential relaxation spectrum is assumed to consist of distinct domains of Debye-type, i.e. exponential character, consistent with heterogeneous dynamics (cf. section 2.3), as depicted in fig. 2.24 .

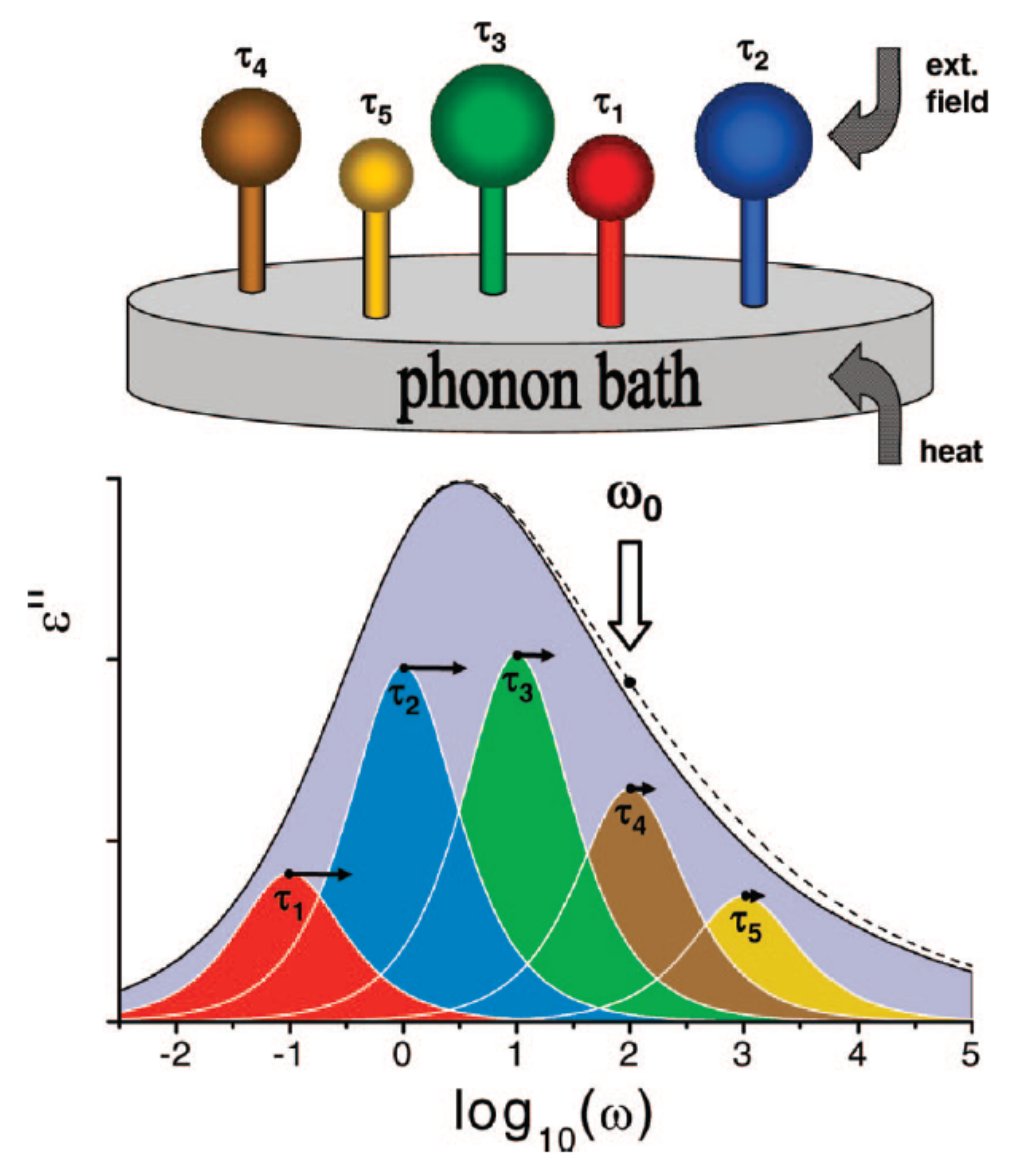

Figure 2.24 Scheme of independent slow modes, weakly coupled to the phonon bath and thus exhibiting slow relaxation of the energy absorbed from an external field. Thus, an increase in effective temperature and a shift in the high-frequency flank of the dielectric loss spectrum are expected [57].

These distinct modes are assumed not to couple with another and to relax independently. If these modes are subject to selective heating, as due to coupling to an electric field of frequency $\nu \approx\left(2 \pi \tau_{i}\right)^{-1}$, they recover the absorbed energy via their coupling to a common phonon bath. It is reflected by the vibrational modes of the system, which inherit half the specific heat $C_{p}$ of the system, while the other half is due to the slow degrees of freedom, i.e. the distinct exponential relaxations. Vibrational modes and the in comparison very slow relaxations exhibit a very weak coupling for the observed temperature range, i.e. relaxations are very weakly coupled to the phonon bath.

The energy, which is transferred irreversibly to the sample, can be expressed as average power $p_{x}$. It depends on the frequency $\nu$ of the external field, its amplitude squared $E_{0}^{2}$, the sample volume $V$, and the dielectric loss susceptibility $\varepsilon(\nu)$. As the energy relaxation to the phonon bath is weak compared to the energy absorption from the external field, the thermal relaxation time $\tau_{T}$ is large and an excess fictive temperature $T_{e}\left(T_{f}=T_{\text {bath }}+T_{e}\right)$ evolves for the selected mode. 
The increase in fictive temperature is related to $\tau_{T}$ via the specific heat $C_{p}$ as

$$
T_{e}=\frac{p_{x}}{\kappa}=\frac{\tau_{T} \cdot p_{x}}{C_{p}},
$$

with the heat conductance $\kappa=C_{p} / \tau_{T}$.

The effect of increased fictive temperature can be quantified as a change of the relaxation time $\tau(T)$ of selected modes by the effective activation energy derived from low fieldmeasurements. The excess temperature can thus be determined by

$$
T_{e}(\tau)=\frac{\varepsilon_{0} E_{0}^{2} \delta \varepsilon}{2 \Delta c_{p}} \frac{\omega^{2} \tau^{2}}{1+\omega^{2} \tau^{2}}
$$

with $\Delta c_{p}$ as the total heat capacity step associated with slow modes, $\varepsilon_{0}$ as permittivity of vacuum, $\delta \varepsilon$ as the dielectric relaxation strength, assuming that the timescale of thermal relaxation is identical to the timescale of polarization relaxation. For modes $\tau>>1 / \omega$ an increase in fictive temperature is observed as a shift of the high frequency flank in fig. 2.23. For modes $\tau<<1 / \omega$ no effect is expected, as experimentally confirmed for many structural glass formers.

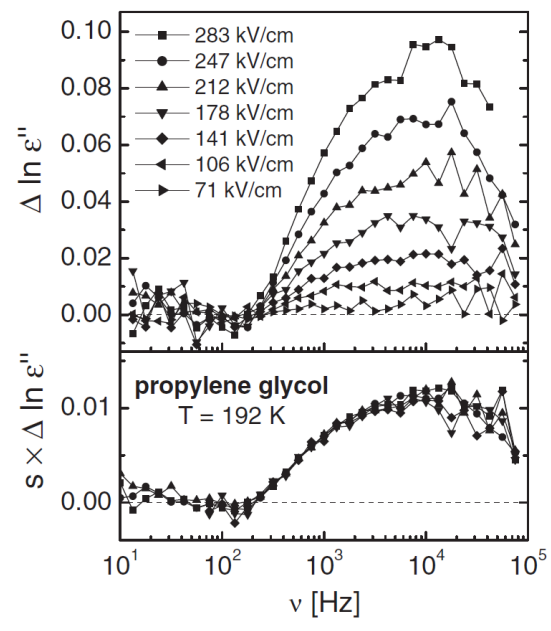

(a)

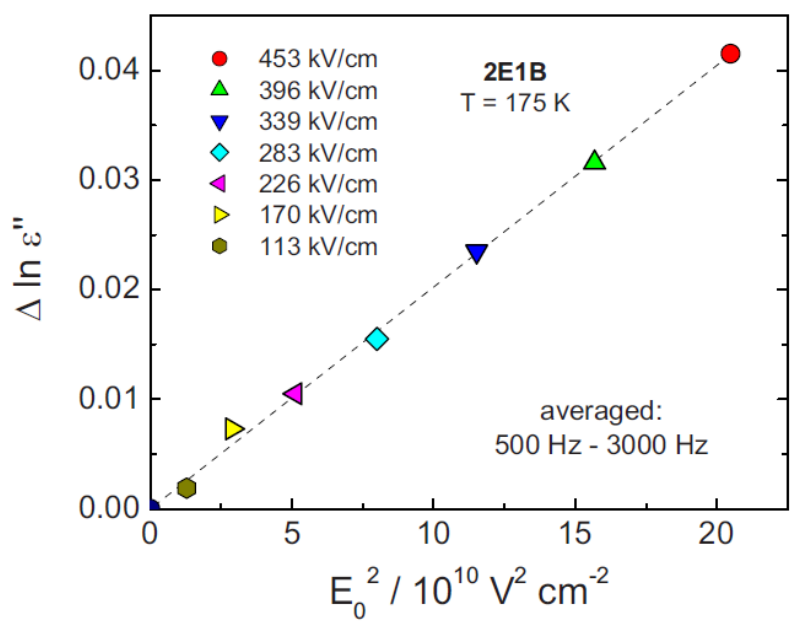

(b)

Figure 2.25 Field amplitude dependence of relative change in dielectric loss $\Delta \ln \varepsilon$ " vs. frequency. (a) for a structural glass-former [148], (b) for a mono-hydroxy alcohol [58].

As eqs. 2.22 and 2.20a state, the shift of the loss spectrum for $\tau \gg 1 / \omega$ due to the energy absorption shows a squared-dependence on the high field amplitude. In the upper part of fig. 2.25a the relative change in dielectric loss, $\Delta \ln \varepsilon^{\prime \prime}=\left(\varepsilon_{h f}^{\prime \prime}-\varepsilon_{l f}^{\prime \prime}\right) / \varepsilon_{l f}^{\prime \prime}$, is plotted against frequency $\nu$ for a structural glass-former. In the lower part, the relative change in dielectric loss is normalized by $s=E_{r e f}^{2} / E_{0}^{2}$ with a common field $E_{r e f}^{2}$, resulting in a mastercurve. This behavior, as well as the plot of $\Delta \ln \varepsilon^{\prime \prime}$ against $E_{0}^{2}$ for a mono-hydroxy alcohol (fig. 2.25b) agrees with the approach via the box-model.

The values of the relative change in dielectric loss $\Delta \ln \varepsilon$ " are deduced from the steady state behavior, after the system has saturated. The change of the loss signal with time is of special interest, as it provides insight into the dynamics of the glass. As the box-model takes the behavior of independent relaxations into account, the case of heterogeneous dynamics would result in a frequency-dependence of the rate needed to achieve steady state. Fig. 2.26 shows the time-dependent behavior of the relative change in loss $\Delta \ln (\tan \delta)$ for various frequencies, which is typical for the behavior of structural glass-formers. It shows a frequency-dependence up to frequencies $\nu$ of the order of $10^{2} \nu_{\max }$, the loss peak frequency for low field amplitude. At higher frequencies, a frequency-independent behavior is observed which is not indicated 
by the box-model. It is related to the isothermal aging of molecular glass-formers, i.e. timeaging time-superposition [103], which is not covered by the model assumptions.

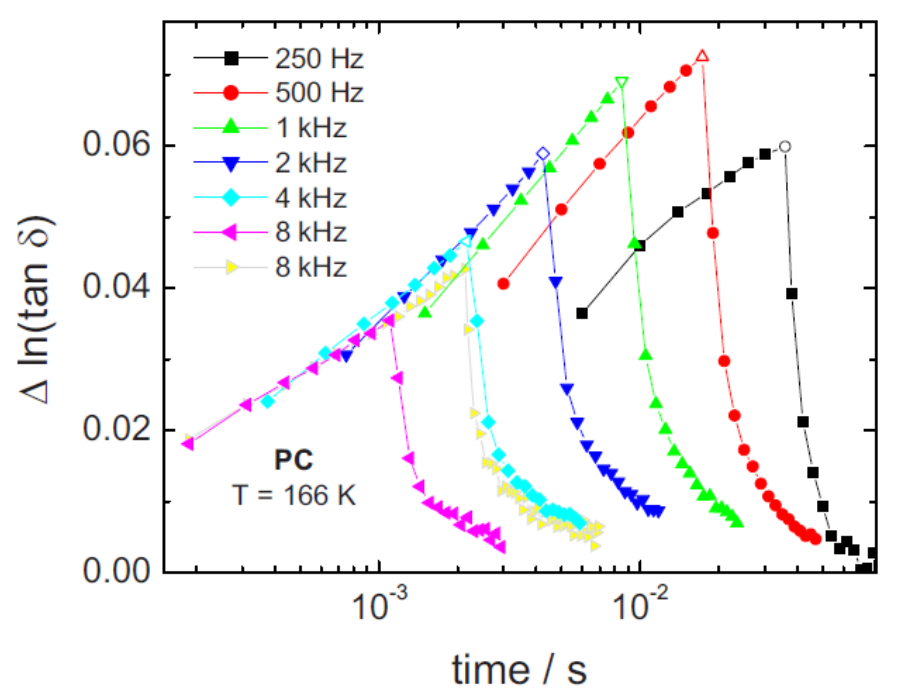

Figure 2.26 Time resolved relative changes in loss factor $\Delta \ln (\tan \delta)$ for various frequencies measured on poly carbonate [58].

Measurements of higher harmonic contributions were performed on several glass-formers, e.g. in [58]. Experimental results by Crauste-Thibierge et al. [23] were connected with a model by Bouchaud and Biroli [16], which uses the response of the third harmonic contribution to gain information on the number of correlated particles $N_{\text {corr }}$ [18]. The model points out that in slow glassy systems under equilibrium conditions the nonlinear response to an external field at the third harmonic probes how dynamics are correlated in space. Thus it is a direct measure of the cooperative length, which is expected to increase as the temperature is reduced. The predictions of the model are experimentally confirmed and extended to several glass-formers [8]. To extract the change of $N_{\text {corr }}$ with temperature, at each temperature $T$ the spectrum of the third harmonic dielectric susceptibility $\chi_{3}(\omega, T)$ is measured. The temperature-dependence of the maximum of the hump is directly correlated to the behavior of $N_{\text {corr }}$ with temperature (cf. fig. 2.27). Richert [115] points out, that the amplitude of the observed hump of the third harmonic susceptibility can also be modeled by assumptions based on a variation of activation energy not including any dynamical correlations or spatial scales.

By the model, higher harmonic contributions to the dielectric susceptibility are associated with an increase in 'amorphous order' while approaching the glass transition [2]. The existence of an underlying phase transition is implied resulting in a state which consists of molecules in 'well-defined yet disordered positions'. Results show a stronger frequency-dependence of $\chi_{5}(\omega)$ than for $\chi_{3}(\omega)$, i.e. the hump in $\chi_{5}(\omega)$ is more pronounced. Moreover $\chi_{5}$ increases stronger with decreasing temperature than $\chi_{3}$, following $\chi_{3} \propto \chi_{5}^{2}$. Thus, the experimental data confirms long-range correlations which act over a length-scale $/$. It is connected to the number of correlated particles $N_{\text {corr }}$ and a timescale $\tau_{\alpha}$. Moreover, the authors claim a confirmation of the thermodynamic signature of a phase-transition which is in contradiction with purely dynamic scenarios as assumed by the box-model.

To extend the realm of nonlinear experiments to metallic glasses, the techniques described above will be transferred to mechanical experiments performed on a Pd-based metallic glass. 


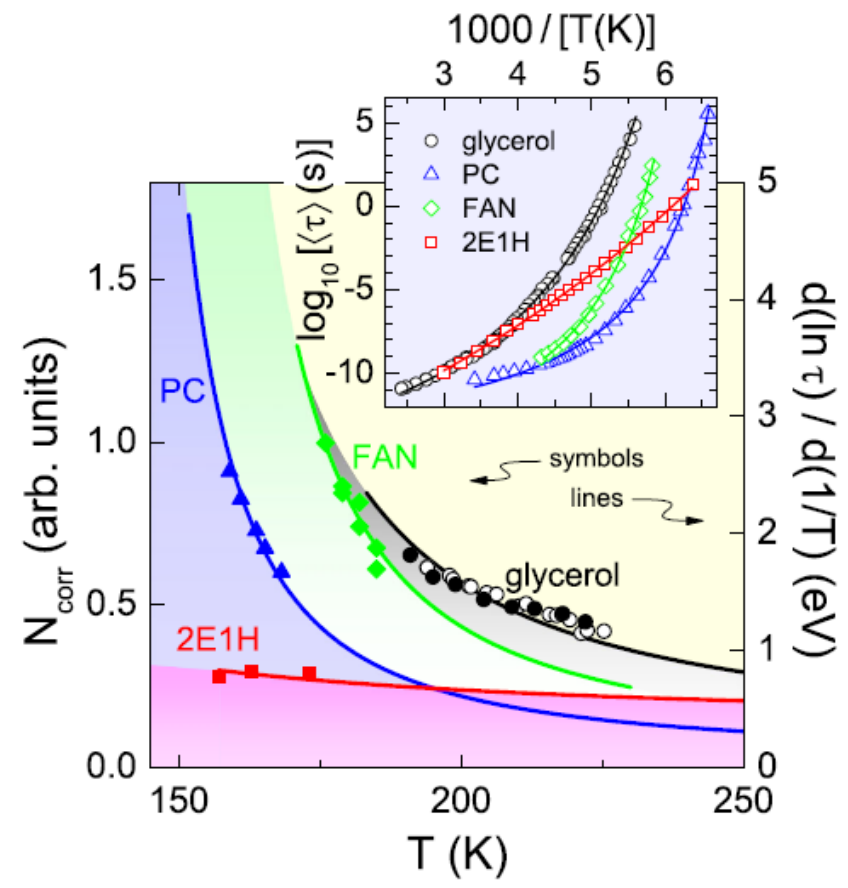

Figure 2.27 Relation between the number of correlated particles $N_{\text {corr }}$ (symbols) and the apparent activation enthalpy (lines), which is adjusted by a normalization factor, against temperature $T$ [8].

\subsubsection{Linear Response and Instantaneous Breakdowns of Elasticity}

As discussed in section 2.5, the occurrence of avalanche dynamics is connected to continuous, linearly increasing segments interrupted by instantaneous drops in the potential energy. If plotting the probability or number of counts of occurring energies dissipated during a drop, double-logarithmic scaling exhibits a linear-like behavior between the energy jumps $\delta E$ and the probability $P(\delta E)$ in accordance to avalanche dynamics theory. In fig. 2.28 the regime exhibiting this power-law behavior extends over roughly three orders of magnitude with exponents, i.e. slopes in log-log scaling, between -0.51 and -0.83 . The data is based on numerical simulations applying quasi-static athermal shear as referred to on $\mathrm{p}$. 15 .

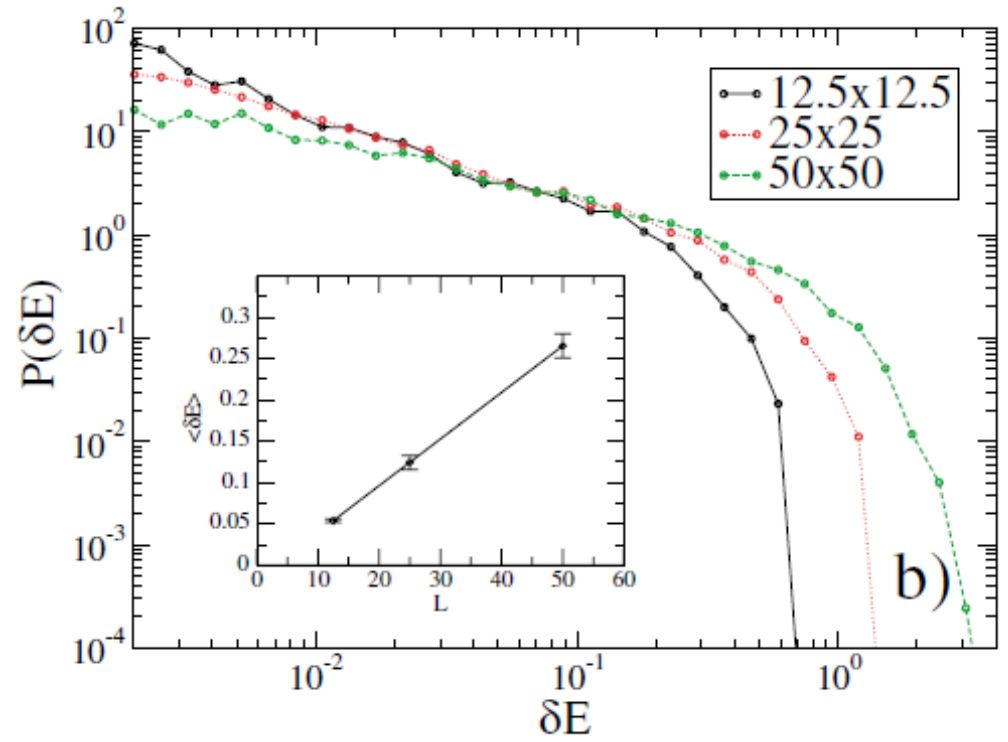

Figure 2.28 Distribution of drops in potential energy $\delta E$ due to microstructural rearrangements based on numerical simulations [88]. 
This is one example on power-law behavior upon many, as it is a widely observed phenomenon and occurs similarly for distributions on the magnitude of earthquakes [24], of Barkhausen noise [106]. The reason for the universality of power-law behavior is due to self-organized critical states and is described by avalanche dynamics theory. It occurs when a system consists of states of minimal stability and small perturbations lead to reorganizations [7]. These occur on very different length scales, leading to a broad distribution of possible values of rearrangements or clusters over some orders of magnitude. It is a state which is also observed under mechanical excitation of amorphous systems, typically connected to the plastic part of the stress-strain-curve, where serrated flow is observed due to shear bands [134]. In the elastic and anelastic regime, the occurrence of STZ $\$$ is postulated as well, and thus under current investigation [54, 77]. Power-law analysis by means of experiments, simulations, and numerical analysis for mechanically excited systems lead to a number of power-law exponents, which are well deduced from experiments in the plastic regime of serrated flow. In case of fig. 2.29, the distribution is extracted by mechanical creep experiments on metallic glass. A dependence of the power-law on the strain rate is suggested, resulting in a value of -0.8 in case of reasonably low strain rates [74, 54]. The basis of evaluation is the extraction of the waiting time, which is connected to the time that is needed to achieve the next strain step, that is defined by the resolution of the strain measurement.

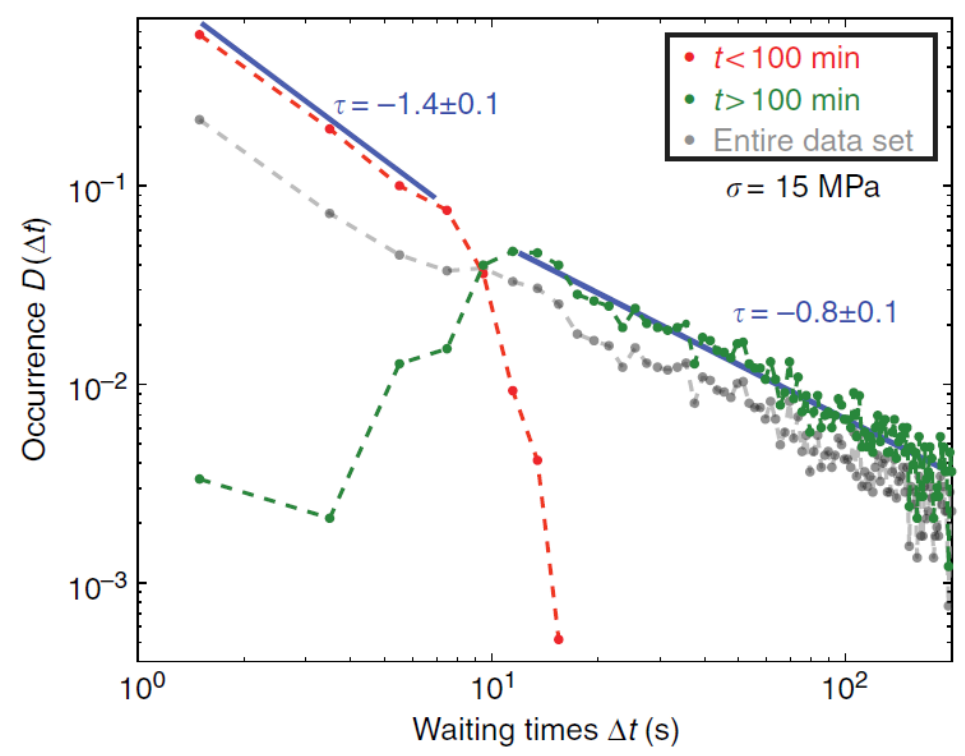

Figure 2.29 Waiting time distribution extracted by tensile creep experiments on $\mathrm{Pd}_{77.5} \mathrm{Cu}_{6.0} \mathrm{Si}_{16.5}$ metallic glass [74].

The appearance of plastic events, observed as drops in stress and potential energy, triggers the question after the exact stress-strain-relation of the continuous segments between events.

The slope of these segments was analyzed using numerical simulations at low temperature under quasi-static straining [32]. Here, the slope of continuous segments is evaluated covering the elastic, anelastic, and plastic regime of stress-strain-curves. It is defined as piecewise linear for the span of a continuous segment with $\Delta \sigma=\mu(\gamma) \Delta \gamma$ with stress $\sigma$, strain $\gamma$, and modulus $\mu$. Three different approaches are realized to gain values for the modulus, i.e. the slope of continuous segments, which are compared in fig. 2.30 .

First, quenched averages of shear modulus are defined by determining the modulus for continuous segments occurring within a certain binning window of strain for an individual stressstrain-curve. Then values of $\mu$ for the same temperature and binning window but from different realizations, i.e. stress-strain-curves, are averaged, while the number of individual values is in the order of $10^{2}$. These values of $\mu$ are defined as $\langle d \sigma / d \gamma\rangle$ and plotted against $\gamma$ in fig. 2.30 as red symbols. 


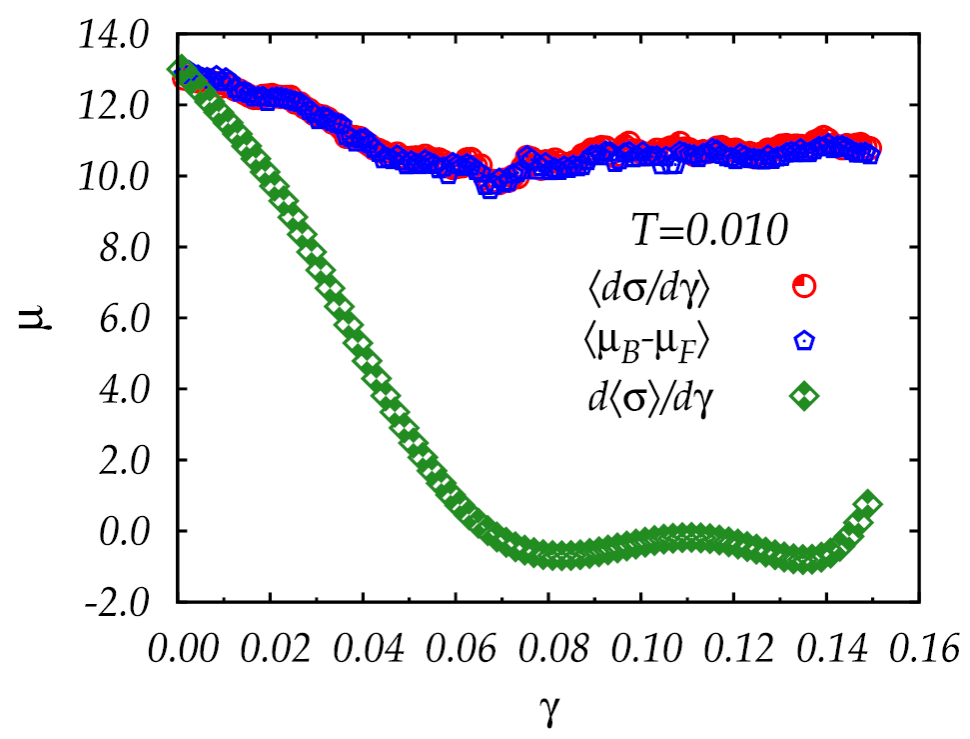

Figure 2.30 Modulus $\mu$ against strain $\gamma$, determined by three different routes as described in the text, based on numerical simulations [32].

Second, the modulus is calculated using the Born approximation $\mu_{B}$ [15] according to

$$
\mu(\gamma)=\mu_{B}(\gamma)-\frac{V}{k_{B} T}\left[\left\langle\sigma^{2}\right\rangle-\left[\langle\sigma\rangle^{2}\right]\right.
$$

with volume $V$, temperature $T$, Boltzmann constant $k_{B}$, as well as $\left\langle\sigma^{2}\right\rangle$ and $\langle\sigma\rangle^{2}$, obtained from averages of the first and second moment of stress from all individual stress-straincurves. Corresponding data is plotted in fig. 2.30 as blue symbols, denoted as $\left\langle\mu_{B}-\mu_{F}\right\rangle$. Third, to obtain annealed averages stress-strain-curves are averaged over many realizations and the local slope, equivalent to the modulus, is measured and plotted in fig. 2.30 as $d\langle\sigma\rangle / d \gamma$ using green symbols.

It is evident that the first and second evaluation approaches yield matching behavior. Thus, the response to mechanical excitation should be considered as piecewise linear, and not in form of a nonlinear expansion. In other words, even for the plastic regime of a stress-strain-

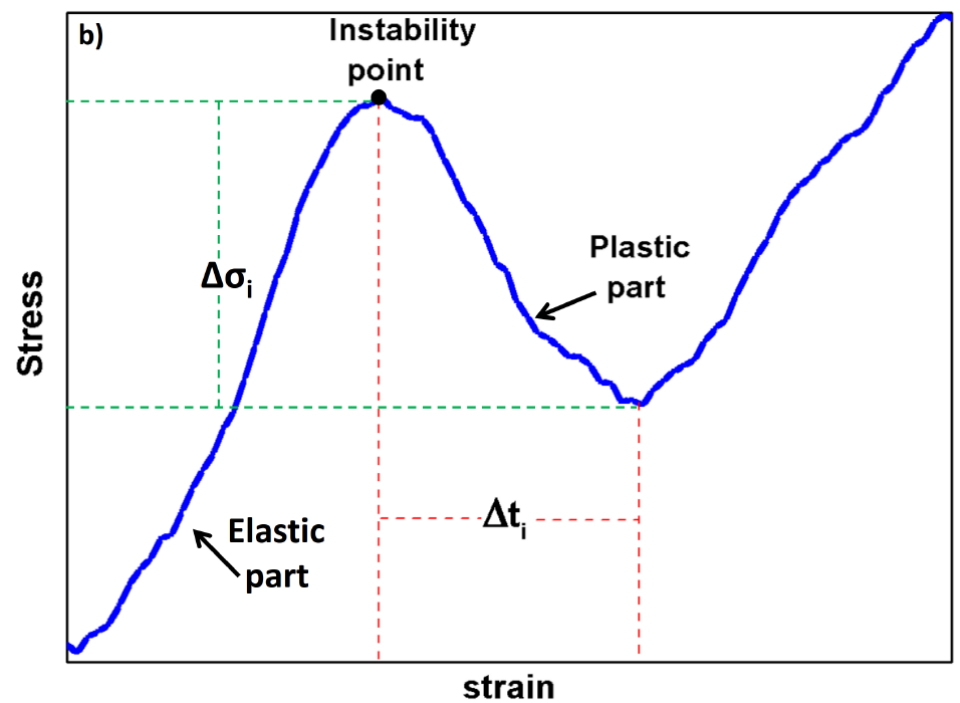

Figure 2.31 Scheme of the characteristic stress-strain-behavior during the EIP-motif, exhibiting initially elastic behavior which is interrupted at the instability point by a stress drop due to a plastic event [77]. 
curve, the elastic modulus is well defined, while the net slope of zero and thus a coarsely defined "modulus" close to zero is due to the counteracting behavior of positive elastic response segments and negative plastic response. This is depicted by the "Elastic-InstabilityPlastic" or (EIP)-motif which consists of a segment of increasing stress, which is elastically coupled to an increasing strain, and a subsequent drop due to a plastic event with inherent energy dissipation. This motif reflects characteristic behavior, which is observed throughout the whole regime of strains, even though with changing intensities regarding occurrence, size $\Delta \sigma$ and duration $\Delta t$.

At higher temperatures, plastic events become indistinguishable as they occur simultaneously in space and time, resulting in superimposed response of events and apparent slopes that might differ from the modulus calculated via the Born term. Thus, higher temperatures exhibit modulus values with similarity to the data based on annealed averages. The authors in [32] state, that a correct measurement of $\mu(\gamma)$ could be obtained by measuring stress fluctuations and an estimation of the Born term, to derive the modulus according to eq. 2.23 . As [140] reports, the rate of an experiment has a strong effect on the occurrence of avalanches. In quasi-static approaches the driving quantity, e.g. stress, strain, or magnetic field is hold at a constant value until the activated avalanches are petered out. Finite driving rates are more realistic as these occur in experiments, for which the effect of superimposed plastic response is enhanced. This temporal overlap of avalanches results in a more complicated response behavior as shown on the example of Barkhausen noise response to increasing sweep rate in fig. 2.32. An avalanche begins when the voltage depicted on the y-axis rises above the base-line voltage and ends when this base-line voltage is achieved again. For small sweep rate the Barkhausen-avalanches are very narrow (A). For higher sweep rates as depicted in (B) and (C), the avalanches exhibit longer durations and more intense behavior, i.e. higher peak voltages. At very high sweep rates as in (D), the avalanches are persistent as the base-line voltage is not approached any more.

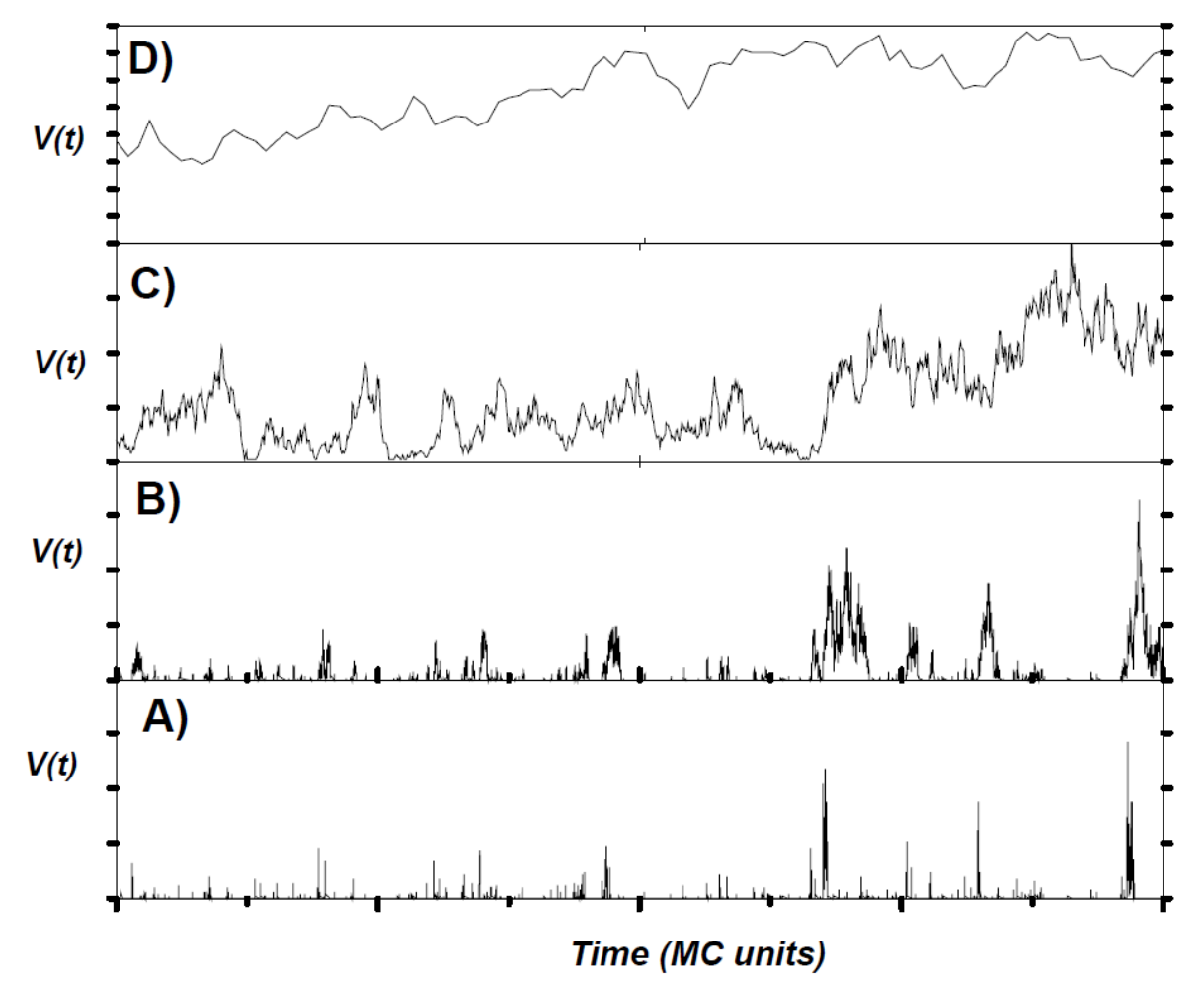

Figure 2.32 Occurrence of Barkhausen noise plotted versus time with increasing sweep rate from A) to D) [140]. 


\section{Chapter 3}

\section{Experimental and Analytical Methods}

In this chapter, experimental methods for the sample characterization and high field measurements by dynamical mechanical excitation are introduced. Further on, analytical methods for data processing are described.

The material that is used for all experiments in this thesis is the metallic glass $\mathrm{Pd}_{40} \mathrm{Ni}_{40} \mathrm{P}_{20}$. It is of high thermal stability in comparison to other glass-formers of this class, as the glass transition temperature $T_{g}$ at $569 \mathrm{~K}$ is separated from the crystallization temperature by roughly $90 \mathrm{~K}$ (based on DSC measurements at $10 \mathrm{~K} \mathrm{~min}^{-1}$ [66]). Moreover it is considered as rather stable towards oxidation under steady Ar-flow, especially in comparison to other metallic glasses. The calorimetric glass transition of $\mathrm{Pd}_{40} \mathrm{Ni}_{40} \mathrm{P}_{20}$ is pronounced and connected to a change in heat capacity $\Delta c_{p}^{s-1}$ of $3.7 \mathrm{cal} \mathrm{mol}^{-1} \mathrm{~K}^{-1}$ [56] between the glassy and the supercooled liquid state due to additional configurational relaxations. To be able to compare this value with the measured change in heat capacity $\Delta c_{p}^{s-1}$, it can be transformed via the molar volume $V_{\mathrm{mol}}$ of $7.68 \mathrm{~cm}^{3} \mathrm{~mol}^{-1}$ and the density $\rho$ of $9.4 \mathrm{~g} \mathrm{~cm}^{-3}$ [102] to SI-units, resulting in a value of $214 \mathrm{~mJ} \mathrm{~g}^{-1} \mathrm{~K}^{-1}$. This is in comparable order as the value for $\Delta c_{p}^{s-1}$ of $290 \mathrm{~m} \mathrm{~J} \mathrm{~g}^{-1} \mathrm{~K}^{-1}$ derived by Wilde et al. [151].

From a mechanical point of view $\mathrm{Pd}_{40} \mathrm{Ni}_{40} \mathrm{P}_{20}$ is considered a ductile material. At room temperature a yield stress $\sigma_{Y}$ of $1600 \mathrm{MPa}$ is reported [96]. The instantaneous shear modulus $G_{\infty}$ at room temperature is determined by means of ultrasonic measurements to 37(1) GPa [66, 102, 79]. A slightly smaller value of $21(1) \mathrm{GPa}$ is reported by Schröter et al. [125], which is determined by torsional rheometry. The connection of elastic and shear modulus via the Poisson ratio (cf. eq. 2.17) of 0.403 [102] allows for a conversion between the two types of moduli. In case of ultrasonic measurements, an elastic modulus of 107(3) GPa is gained, rheological experiments yield a value of 59(3) GPa. The elastic compliance is thus $9.3(3) \times 10^{-12} \mathrm{~Pa}^{-1}$ for the former, and $16.9(9) \times 10^{-12} \mathrm{~Pa}^{-1}$ for the later experimental method.

The fragility $m$ of $\mathrm{Pd}_{40} \mathrm{Ni}_{40} \mathrm{P}_{20}$ is determined to 41.5 measured by dynamical mechanical analysis at $1 \mathrm{~Hz}$ [125]. This gives a KWW-exponent $\beta_{K W W}$ of $0.65(9)$ according to eq. 2.10 . The value of $\beta_{K W W}=0.65$ is used for KWW-fits in the following chapters. VFT-parameters are $\eta_{0}=5 \times 10^{6} \mathrm{Pas}, B=D \cdot T_{V F T}=690 \mathrm{~K}$ and $T_{V F T}=500 \mathrm{~K}$ according to [151].

\subsection{Preparation of Metallic Glass Samples}

To prepare $\mathrm{Pd}_{40} \mathrm{Ni}_{40} \mathrm{P}_{20}$ metallic glass samples, pure Palladium (Heraeus, bar, purity $3 \mathrm{N5}$ ) and pure Nickel (Alfa Aesar, lump, purity 3N7) are weighed to an accuracy of $0.1 \%$ and melted several times in an arc melter in presence of Zirconium as an oxidant to gain a prealloy. Phosphorus (Chempur, purity 5N) is weighed under Argon-atmosphere (Air Liquide, purity $5 \mathrm{~N}$ ) to an accuracy of $0.1 \%$ with an addition of $3 \%$ in weight to the amount necessary 
to get the correct composition for the pre-alloy. Together with Boron-oxide $\mathrm{B}_{2} \mathrm{O}_{3}$ (ChemPur, purity $4 \mathrm{~N}$ ), the pre-alloy and the Phosphorus are sealed under a protective atmosphere of 750 mbar Argon into a quartz-tube. The quartz tube is positioned within an induction coil and heated until a homogeneous melt is gained, which is rapidly cooled in a bucket of water. In order to prepare the metallic glass, the alloy is chopped into pieces sufficiently small to fit into a quartz tube using a hardened steel mortar. The quartz tube, providing an injection hole of $0.8 \mathrm{~mm}$ in diameter, is mounted in the vacuum chamber of a melt spinner, centered within an induction coil. The quartz tube is connected by a valve to a pressurized gas tank of Argon (Air Liquide, purity $5 \mathrm{~N}$ ) and is located above a copper form of low oxygen content, which is cooled by a continuous flow of liquid nitrogen. Chamber and pressure tank are rinsed several times by successive evacuation and purging with Argon gas. After the final evacuation, a minimal pressure of $10^{-7} \mathrm{mbar}$ is achieved. To prepare the shot, the atmosphere in the chamber is set to $100 \mathrm{mbar}$ Argon pressure while the gas tank is adjusted to $900 \mathrm{mbar}$ Argon pressure. The generator is set to a value of 5.0 to 5.5 to heat the alloy up to temperatures of $1800 \mathrm{~K}$ to $2200 \mathrm{~K}$ via the induction coil. During the shot the molt is blown into the copper form by abruptly opening the valve to the pressure tank. As soon as the molt fills the copper-mold, it is quenched by a rate of roughly $10^{5}$ to $10^{6} \mathrm{~K} \mathrm{~s}^{-1}$ to a metallic glass [149]. After separation of the sample from the copper form, a metallic glass sample of $30 \mathrm{~mm}$ length, $10 \mathrm{~mm}$ width, and $1 \mathrm{~mm}$ thickness is obtained. As a preparation for mechanical experiments, bars of $1 \mathrm{~mm}$ width are cut from the sample using an annular diamond saw, resulting in samples of $1 \mathrm{~mm} \times 1 \mathrm{~mm} \times 30 \mathrm{~mm}$.

\subsection{X-Ray Diffraction}

The amorphous state of the samples is probed by X-Ray diffraction (XRD) using a D 8 by Bruker Analytical X-Ray Systems with a $\mathrm{Cu} \mathrm{K}_{\alpha}$-cathode with a wavelength $\lambda$ of $157.4 \mathrm{pm}$. $A \Theta-2 \Theta$-setup is used which consists of the $x$-ray source, sample, and detector. Detector and sample are moved in such a way, that only those $\mathrm{x}$-rays are detected which are scattered by twice the incident angle $\Theta$ relative to the sample-source-plane. The condition for constructive interference is fulfilled by Bragg's Law

$$
2 d \sin \theta=n \lambda,
$$

where $n$ is the order of the observed maximum. As there are no crystallographic planes in a disordered system, only atoms at a certain distance $d$ can contribute to a constructive interference phenomenon. Thus, the signal is much less intense than for a crystalline material. Moreover, there are no peaks observable, but rather widely spread maxima due to the broad distribution of atomic distances [38].

Slits behind source and sample are chosen to a width of $0.6 \mathrm{~mm}$, the slit in front of the detector has a width of $1.0 \mathrm{~mm}$. The amorphous samples are analyzed in an angular range of $\Theta$ from $20^{\circ}$ to $90^{\circ}$ in order to get an overview over the first and second amorphous maximum. Therefore, continuous scans with a rate of $20^{\circ} \mathrm{min}^{-1}$ and a step size of $0.1^{\circ}$ are applied. Furthermore, a detailed scan in the regime of the first amorphous maximum is performed as a step scan with a rate of $10 \mathrm{~s}$ per step and a step size of $0.05^{\circ}$. Samples are analyzed systematically on both the cut and the cast surface.

\subsection{Energy Dispersive X-ray Spectroscopy}

To analyze the chemical composition of the samples, a scanning electron microscope (SEM) by Carl Zeiss NTS GmbH of type LEO SUPRA 35 is used in combination with an energy dispersive X-Ray spectroscopy (EDX) system by Thermo Fisher Scientific GmbH. 
The high energy electrons of the SEM beam excite electrons from the electron shells of the sample material's atoms. Vacant electron sites in lower shells are reoccupied by electrons from shells of a higher energy ground state. This process is accompanied by a release of photons with a characteristic wavelength depending on the atomic species, which is in the regime of $\mathrm{x}$-rays. If these photons collide with the $\mathrm{Si}(\mathrm{Li})$-detector, electron-hole-pairs are generated. As their number is proportional to the energy of the absorbed photon, its wavelength can be measured by an integration over the current which flows due to the free charge carriers excited by the photon with respect to time [60].

The EDX analysis was performed on five different sites of a pristine sample of each sample cast. Each sample was attached to an Aluminum holder by a carbon-based glue-pad and inserted into the SEM. The samples were measured under $20 \mathrm{kV}$ acceleration voltage and the area contributing to the EDX spectrum corresponds to the scanned area under $1 \mathrm{k}$ magnification. EDX peaks resulting from material used for mounting the sample, as Aluminum and Carbon, are excluded from the quantification, as well as oxygen and silicon, which was found in few spectra and accounts for less than $1 \%$ at. in the quantification. The contributions of elements included in the spectra is taken into account to deduce the average quantification for all samples.

\subsection{Differential Scanning Calorimetry}

To measure the calorimetric response of $\mathrm{Pd}_{40} \mathrm{Ni}_{40} \mathrm{P}_{20}$ and the onset of the glass transition in particular, a DSC 7 by Perkin Elmer was used. It consists of two separate furnaces, one for heating an Aluminum pan holding the sample, the other for heating the empty Aluminum pan that works as a reference. Two Platinum wires are used as thermoelements, one for each pan. The heating protocol dictates a constant heating rate for both furnaces and the differential heat flow necessary to accomplish this is measured under the assumption that the Aluminum pans are identical in both furnaces. This way, ideally, the differential heat flow signal is only due to the heat necessary for increasing the temperature of the sample material. Exothermic sample behavior creates thus a low differential heat flow, endothermic behavior a rather high differential heat flow. The Aluminum pans in the furnaces are purged with Argon gas to enhance the thermal coupling between pans and furnaces. As a preparation for temperature scans, the system is calibrated by scans of Indium and Zinc. For both materials the onsets of melting are measured as well as the melting enthalpy of Indium. The results are compared to literature data, and used for rescaling heat flow and temperature.

Measurements of metallic glass samples were performed in the range of $320 \mathrm{~K}$ to $820 \mathrm{~K}$ at a heating rate of $10 \mathrm{~K} \mathrm{~min}^{-1}$. The amount of sample material is in the order of $20 \mathrm{mg}$. To obtain the contributions to the specific heat which are due to the amorphous material only, the samples are measured twice in the above stated temperature regime and the second run is subtracted from the first. As crystalline and glassy specific heat is of comparable order (cf. fig. 2.1a), the resulting difference in specific heat shows values close to zeros for temperatures below $T_{g}$, followed by a pronounced glass transition and the dip due to latent heat during crystallization. The onset of the glass transition is analyzed by using the doubletangential method [121], which is based on linear regressions $y_{g}$ in the glassy temperature regime and $y_{t}$ in the regime of the glass transition with:

$$
y_{g}=m_{g} \cdot T+b_{g} \quad \text { and } \quad y_{t}=m_{t} \cdot T+b_{t}
$$

The abscissa of the intersection point of the fitting functions $y_{g}$ and $y_{t}$ is given by

$$
S=\frac{b_{g}-b_{t}}{m_{t}-m_{g}}
$$

and is equivalent to the experimental glass transition temperature $T_{g}^{o n}$. An example for the application of the double tangential method is shown in fig. 4.3 . 


\subsection{Dynamical Mechanical Analysis}

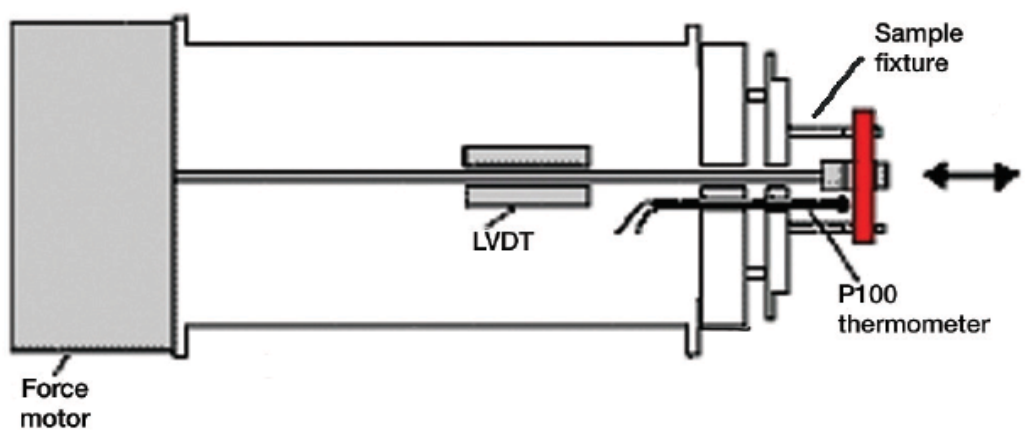

Figure 3.1 Scheme of the DMA 8000 setup [39].

With a dynamical mechanical analyzer (DMA) by Perkin Elmer of type DMA 8000, an oscillatory excitation is applied to a sample to measure the response of the sample. From this the loss and storage modulus is determined, as well as the loss factor (cf. section 3.6).

The core of the DMA is the force motor. It drives the movable axis to apply a sinusoidal or constant force to the sample (see fig. 3.1), which is clamped to its front end. The position of the movable axis and thus the sample is measured by a linear variable differential transformer (LVDT) It consists of a primary coil and two secondary coils, which are attached to the body of the DMA and enclose the primary coil. They are located close to the movable axis, to which a ferromagnetic material is attached. It is positioned in the very center of the coils if the movable axis is in the neutral position. When the movable axis is displaced, the ferromagnetic material enhances differing inductive fields in the secondary coils. This difference in induction voltage is linearly connected to the displacement of the movable axis and gives a reliable measure of the sample movement in a broad temperature and frequency regime [92, 105]. For the analysis of the temperature dependent mechanical behavior, a heating device can be installed covering the front of the DMA including the sample. It is purged by Argon gas (Air Liquide, purity $5 \mathrm{~N}$ ) to reduce the oxidation of the probed material. The range of parameters that can be inititalized by the DMA 8000 parameters are given in table 3.1] [105].

Measurements are performed in single cantilever mode. One end of the sample is stationarily fixed between two Titanium clamps, the other end is similarly clamped to the movable axis and can thus be addressed by a well defined force by the force motor (see fig. 3.2). Always, the sample is mounted with the cut surface perpendicular to the direction of displacement, even though no difference between the mechanical response of the cut and the cast surface was observed during test measurements.

\begin{tabular}{ll}
\hline & Accessible Parameter Range \\
\hline Temperature $T$ & $80 \mathrm{~K}$ to $670 \mathrm{~K}$ \\
Force $F$ & $\pm 10 \mathrm{~N}$ \\
Displacement $\delta$ & $\pm 1 \mathrm{~mm}$ \\
Frequency $f$ & $10^{-2} \mathrm{~Hz}$ to $3 \times 10^{2} \mathrm{~Hz}$ \\
\hline
\end{tabular}

Table 3.1 Accessible range of parameters for DMA 8000 according to page 111 in [105].

To be able to calculate strain $\epsilon$ and stress $\sigma$ for each sample, the sample dimensions are measured using a micrometer caliper with an accuracy of $1 \mu \mathrm{m}$. Therefore, five values for each the width (perpendicular to cast surfaces) and the thickness (perpendicular to cut surfaces, i.e. parallel to the movable axis) are measured. The minimum value is used for stress 
and strain calculations, as the sample is addressed most effectively in its most narrow crosssectional regime by the applied force. The length of the sample is set in the order of $10 \mathrm{~mm}$. Its accurate value is measured after mounting the sample and is determined as the mean of three values, which are taken from the middle position between clamps, where the sample is ideally located, and slightly below and above the sample. To ensure that the clamps are parallel towards another, the top and bottom distance between clamps is checked and adjusted if differing by more than $0.02 \mathrm{~mm}$.

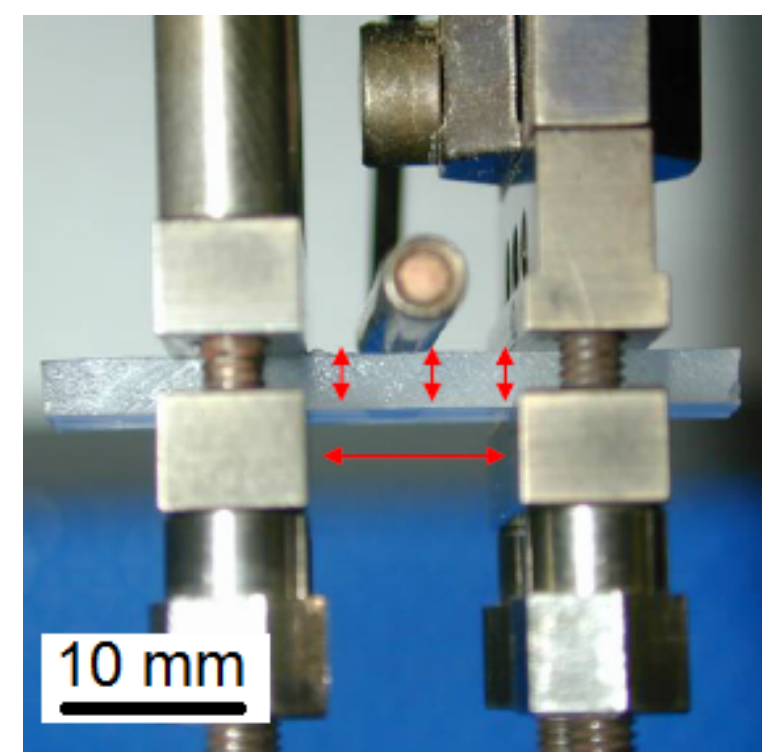

Figure 3.2 Scheme of the sample mount in single cantilever mode in the DMA 8000 [40]. The three short arrows indicate the sample thickness, while the long arrow depicts the sample length.

Depending on the intended measurement protocol, two different programs can be used to address the DMA 8000 to apply a force. One of them is the commercial program provided by Perkin Elmer, which allows for setting up standard measurement protocols, e.g. temperature scans. Heating rate, time intervals at constant elevated temperatures, constant and sinusoidal forces can be adjusted according to the aims of the experiment.

To be able to deviate from these standard protocols, a C-Sharp program is used, further on referred to as large amplitude oscillatory spectroscopy (LAOS) -program [44], which addresses the DMA 8000 directly and transfers a force protocol with $1 \mathrm{~ms}$ resolution in time via a waveform generator to the external input of the analyzer. In this case, the hardware connection between the computer with the DMA-program and the DMA 8000 is severed and the commercial program does neither control nor influence force or displacement. Only the temperature protocol can be operated using the commercial program. The separation of force and strain control from the DMA-program is necessary as otherwise additional frequencies add to the signal, which evolve due to the analyzer's circuitry, and which interfere with the evaluation by Fourier-analysis. The output is directly picked up from the LVDT via an analog-digital-transformer. One data point per millisecond is saved and stored on an external computer. By calibration of the LVDT-signal, a distinct displacement can be calculated. This way, the commercial program can be used for adjustments in temperature, while the LAOS program dictates the amplitude and form of the applied force.

To keep the DMA in an operational state, several calibrations are performed on a regular basis. The most frequent one is the 'Zero Force' calibration, which restores the movable axis to its true zero position and stores the value necessary to perform the re-balance (cf. [105]), and is performed before every measurement. This calibration is active when measured with the DMA-program, but is not applied during measurements with the LAOS-program. More detailed concepts of the calibration of offset and temperature are given in the appendix. 


\subsubsection{Temperature Scans and Static Stress-Strain-Curves}

Temperature scans are performed using the Perkin Elmer program in a range from $300 \mathrm{~K}$ to ca. $590 \mathrm{~K}$ at a frequency of $1 \mathrm{~Hz}$. Small displacement amplitudes between $1 \mu \mathrm{m}$ and $5 \mu \mathrm{m}$ are applied to characterize the different sample casts. Properties as the modulus $E$, the phase shift between force and displacement $\delta$, and the onset of the glass transition $T_{g}^{o n}$ were determined by these scans.

At a constant temperature the relation between static stress and static strain is measured using the Perkin Elmer program. The maximum value of stress is defined as the upper limits in force at $10 \mathrm{~N}$. The size of stress steps is defined by the force rate, which is $0.5 \mathrm{~N} \mathrm{~min}^{-1}$. The experiment is terminated if either the maximum force is established or if the maximum displacement of $1 \mathrm{~mm}$ is achieved.

\subsubsection{Large Amplitude Oscillatory Spectroscopy}

The protocol of LAOSexcitation is schematically shown in fig. 3.3. It aims on the measurement of the time-dependent influence of high sinusoidal stress fields on mechanical properties of metallic glasses. The excitation is due to a sinusoidal force $F(t)=F_{0} \cdot \sin (2 \pi f t)$. The experiment starts with a relatively small force amplitude $F_{0}$ which is applied for 3600 periods. It is schematically depicted in fig. 3.3 as the blue sine waves during the first part of the experiment which is referred to as 'initial low field'. It is followed by the high field excitation for 3600 periods, which is indicated by an increase in force amplitude by a factor of five(red lines, 'high field'). Finally the excitation amplitude is decreased again to the initial low field value and persists for another 3600 periods. It is depicted as blue lines again, and referred to as 'second low field'. This way, each experiment lasts for $3 \cdot 3600$ periods at a frequency $f$ of $1 \mathrm{~Hz}$, resulting in $3 \mathrm{~h}$ duration under a data acquisition rate of $1 \mathrm{~ms}^{-1}$.

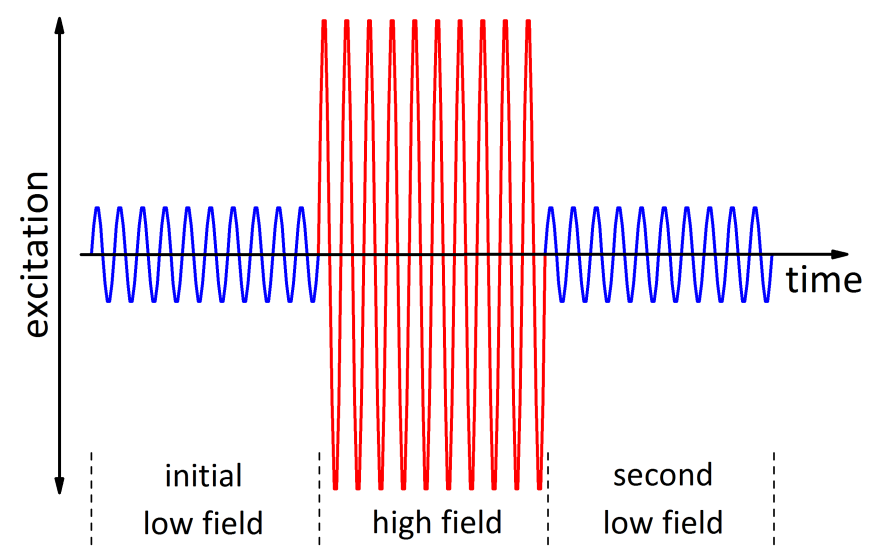

Figure 3.3 Scheme of the LAOS excitation protocol.

To equilibrate the sample thermally to a steady state before the LAOS protocol is performed, the commercial program is used to control the thermal conditions. Therefor the sample is heated to the temperature at which the experiment will be executed. At this temperature, the sample is annealed for $60 \mathrm{~min}$ before the LAOS-program is started. Throughout the protocol described above the temperature is kept constant and the sample chamber is purged with Argon gas to avoid sample oxidation. 


\subsection{Nonlinear Analysis}

In order to quantify the nonlinear contributions, which are expected to occur in mechanical experiments under high field excitation, the response is analyzed regarding its nonlinear components. This is accomplished by a period-by-period Fourier-analysis, giving quantitative information on the response at the fundamental and higher harmonic frequencies. These frequencies are individually connected to a nonlinear coefficient, which gives a measure for the extent of nonlinearity at a given stress. The equations for the linear and nonlinear relation between stress $\sigma$ and strain $\varepsilon$ are explicitly stated in the following section. The procedure to determine this global degree of nonlinearity from the experimental data is described in detail regarding the Fourier-analysis and the fitting of the data by a squared KWW-function.

\subsubsection{Nonlinear Response to Mechanical Excitation}

In the case of an exciting sinusoidal strain of angular frequency $\omega$, the strain and the responding stress can be described as:

$$
\begin{aligned}
& \widehat{\varepsilon}=\varepsilon_{0} \cdot e^{i\left(\omega t-\delta_{\varepsilon}\right)} \\
& \hat{\sigma}=\sigma_{0} \cdot e^{i\left(\omega t-\delta_{\sigma}\right)}
\end{aligned}
$$

In the case of a linear excitation, the complex modulus $\hat{E}$ can be determined from stress and strain according to Hook's Law as follows:

$$
\hat{E}=\frac{\hat{\sigma}}{\widehat{\varepsilon}}=\frac{\sigma_{0}}{\varepsilon_{0}} \cdot \frac{e^{i\left(\omega t-\delta_{\sigma}\right)}}{e^{i\left(\omega t-\delta_{\varepsilon}\right)}}=\frac{\sigma_{0}}{\varepsilon_{0}} \cdot e^{i\left(\delta_{\varepsilon}-\delta_{\sigma}\right)}=|\hat{E}| \cdot e^{i \delta}
$$

Via the phase $\operatorname{lag} \delta$ between stress $\sigma$ and strain $\varepsilon$, the storage modulus $E^{\prime}$ and the loss modulus $E^{\prime \prime}$ are related at a given frequency in accordance to the Kramers-Kronig-relations [26, 71, 109] and are connected by:

$$
\begin{gathered}
\hat{E}=E^{\prime}+i E^{\prime \prime} \\
E^{\prime}=|\hat{E}| \cdot \cos (\delta) \\
E^{\prime \prime}=|\hat{E}| \cdot \sin (\delta)
\end{gathered}
$$

The storage modulus describes to which extent the energy absorbed by the sample is stored reversibly in the system. The loss modulus quantifies how much energy is irreversibly lost or dissipated to the sample. The ratio between storage and loss modulus is defined as the loss, also loss factor, $\tan \delta$.

$$
\tan \delta=E^{\prime \prime} / E^{\prime}
$$

The relation between stress $\sigma$ and strain $\varepsilon$ to force $F$ and deformation $k$ depends on the geometry of the sample and the setup of the measurement, but can be expressed generally by the geometry factor $b$.

$$
\frac{\sigma_{0}}{\varepsilon_{0}}=\frac{F_{0}}{k_{0}} \cdot \frac{1}{b}
$$

In case of a tension experiment the geometry factor is set to $\frac{x \cdot y}{z}$, with width $x$, thickness $y$, and length $z$ of the sample. In a single cantilever experiment, as performed in this study, the geometry factor is set to $\frac{x(y / z)^{3}}{1+2.9(y / z)^{2}}$ [105]. 
Thus, for single cantilever experiments, the stress amplitude $\sigma_{0}$ can be calculated from the force amplitude $F_{0}$ via the stress factor $b_{\sigma}$ :

$$
\sigma_{0}=b_{\sigma} \cdot F_{0}=\frac{\left(x y^{2} / z\right)}{3\left(1+2.9(y / z)^{2}\right)} \cdot F_{0}
$$

The strain amplitude $\varepsilon_{0}$ can similarly be calculated from the displacement amplitude $k_{0}$ via the strain factor $b_{\varepsilon}$ :

$$
\varepsilon_{0}=b_{\varepsilon} \cdot k_{0}=3 \frac{y}{z^{2}} \cdot k_{0}
$$

In case of a stress-driven experiment it is convenient to define the compliance $\hat{\chi}^{(1)}$ as the inverse modulus of the system:

$$
\widehat{\varepsilon}=\frac{1}{\hat{E}} \cdot \hat{\sigma}=\hat{\chi}^{(1)} \cdot \hat{\sigma}
$$

Thus:

$$
\hat{\chi}^{(1)}=\hat{E}^{-1}=\frac{\varepsilon_{0}}{\sigma_{0}} \cdot e^{-i \delta}=\chi^{\prime(1)}-i \chi^{\prime \prime(1)}
$$

Storage and loss compliances $\chi^{\prime}$ and $\chi$ " can be written as:

$$
\begin{gathered}
\chi^{\prime(1)}=\frac{\varepsilon_{0}}{\sigma_{0}} \cdot \cos (-\delta)=\frac{\varepsilon_{0}}{\sigma_{0}} \cdot \cos (\delta) \\
\chi^{\prime \prime(1)}=\left|\frac{\varepsilon_{0}}{\sigma_{0}} \cdot \sin (-\delta)\right|=\left|-\frac{\varepsilon_{0}}{\sigma_{0}} \cdot \sin (\delta)\right|=\frac{\varepsilon_{0}}{\sigma_{0}} \cdot \sin (\delta)
\end{gathered}
$$

With increasing stress, the stress-strain-curve can be described by a nonlinear expansion. Due to the point-symmetry around the origin, it consists of terms of odd order, similar to approaches in dielectric spectroscopy as in eq. 2.19 based on [113]:

$$
\varepsilon=\chi^{(1)} \sigma+\chi^{(3)} \sigma^{3}+\chi^{(5)} \sigma^{5}+\ldots
$$

with a nonlinear compliance $\chi^{(i)}$ for odd orders $(i)$. The stress applied during the experiment is of a sinusoidal form with peak amplitude $\sigma_{0}$.

$$
\sigma=\sigma_{0} \cdot \sin (\omega t)
$$

Substitution of this equation into eq. 3.14 yields

$$
\varepsilon / \sigma_{0}=\chi^{(1)} \cdot \sin (\omega t)+\chi^{(3)} \cdot \sigma_{0}^{2}(\sin (\omega t))^{3}+\chi^{(5)} \cdot \sigma_{0}^{4}(\sin (\omega t))^{5}+\ldots
$$

With the trigonometric relations

$$
(\sin (x))^{3}=\frac{3 \sin (x)-\sin (3 x)}{4}
$$

and

$$
(\sin (x))^{5}=\frac{10 \sin (x)-5 \sin (3 x)+\sin (5 x)}{16}
$$

and under consideration of individual nonlinear coefficients, the nonlinear expansion can be written as [138]:

$$
\begin{array}{r}
\varepsilon / \sigma_{0}=\chi^{(1)} \cdot \sin (\omega t)+\frac{3}{4} \chi_{1}^{(3)} \sigma_{0}^{2} \sin (\omega t)+\frac{5}{8} \chi_{1}^{(5)} \sigma_{0}^{4} \sin (\omega t) \\
-\frac{1}{4} \chi_{3}^{(3)} \sigma_{0}^{2} \sin (3 \omega t)-\frac{5}{16} \chi_{3}^{(5)} \sigma_{0}^{4} \sin (3 \omega t) \\
+\frac{1}{16} \chi_{5}^{(5)} \sigma_{0}^{4} \sin (5 \omega t)
\end{array}
$$

Now, the nonlinear contributions can be expressed relatively to the linear compliance $\chi^{(1)}$ for the first harmonic $\omega$ as well as higher harmonic contributions. These quantities will be extracted from the experimental data to describe the nonlinear behavior of the metallic glass $\mathrm{PdNiP}$ in single cantilever bending experiments under high stress amplitude. 
For the first harmonic, i.e. at the fundamental frequency $\omega$, the total compliance reads:

$$
\left(\varepsilon / \sigma_{0}\right)_{\omega}=\chi^{(1)}+\frac{3}{4} \chi_{1}^{(3)} \sigma_{0}^{2}+\frac{5}{8} \chi_{1}^{(5)} \sigma_{0}^{4}
$$

By subtraction of and division by the linear compliance $\chi^{(1)}$, the purely nonlinear part of the first harmonic can be extracted. It reads including terms up to the third order in the expansion from eq. 3.14

$$
\Delta \ln \left(\varepsilon / \sigma_{0}\right)_{\omega}=\frac{3}{4} \frac{\chi_{1}^{(3)}}{\chi^{(1)}} \sigma_{0}^{2}=\frac{\left(\varepsilon / \sigma_{0}\right)_{\omega}-\chi^{(1)}}{\chi^{(1)}}
$$

Division of eq. 3.21 by $\sigma_{0}^{2}$ yields:

$$
\frac{\Delta \ln \left(\varepsilon / \sigma_{0}\right)_{\omega}}{\sigma_{0}^{2}}=\frac{3}{4} \frac{\chi_{1}^{(3)}}{\chi^{(1)}}
$$

For the third harmonic contribution follows:

$$
\frac{\left(\varepsilon / \sigma_{0}\right)_{3 \omega}}{\chi^{(1)}}=-\frac{1}{4} \frac{\chi_{3}^{(3)}}{\chi^{(1)}} \sigma_{0}^{2}
$$

\subsubsection{Fourier Analysis of LAOS Measurements}

To extract the nonlinear components from the experimental data, a Fourier-analysis is performed. It gives the basis to establish a time-dependent analysis of phase and amplitude signals for both force and displacement. Therefore, a program written by Prof. Ranko Richert is used which analyzes the signal period-by-period at the fundamental frequency $\omega$ and higher harmonic frequencies $2 \omega, 3 \omega, \ldots$ as desired up to the ninth harmonic, similar as in [57]. The data enters the program in form of a text-file containing three columns in total. The first column contains the time step, the second one the exciting force, and the third column enters with the measured displacement. The data output file contains $4 n+2$ values per analyzed period, with $n$ being the number of evaluated frequencies. For each frequency, the amplitude and phase shift for both excitation and response signal is given, another two values add for the offsets in force and displacement. From the results of the Fourier-analysis the period-wise stress, strain, modulus, and compliance data is calculated for the fundamental and higher order frequencies. The calibration is based on the calibration file used by the commercial Perkin Elmer program combined with adjustments to adapt to the Fourieranalyzed data. According to the derivation in the previous section, nonlinear contributions are extracted as shown in chapter 5 .

\subsubsection{Fit of Time-Dependent Behavior}

To evaluate the time dependence of the response to high field excitation, a KWW function is used based on the description in section 2.9. Due to the squared dependence of the nonlinear contribution to the fundamental frequency on the stress amplitude $\sigma_{0}$ according to eq. 3.21 the time-dependent behavior can be described by a KWW function with a squared body function [155, 10], as in fig. 3.4. To fit the time-dependent behavior of a quantity $X$ in the case of mechanical retardation under high field, the KWW-function is written as:

$$
X=A_{h f}+B_{h f}\left(1-\exp ^{-(t / \tau)^{\beta_{K} w w}}\right)^{2}
$$




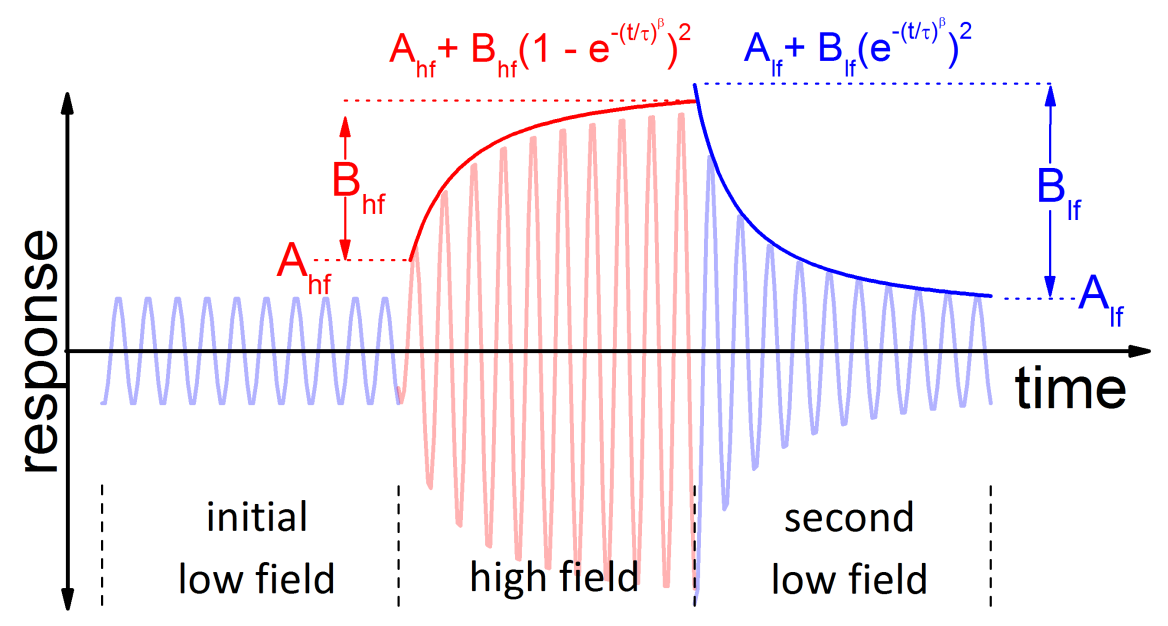

Figure 3.4 Scheme of oscillating response with squared KWW-fits for high field and second low field.

The relaxation during the second low field is assumed by:

$$
X=A_{l f}+B_{l f}\left(\exp ^{-(t / \tau)^{\beta_{K} w w}}\right)^{2}
$$

The squared dependence on $\sigma_{0}$ is characteristically observed as an apparently longer timescale during high field, and a comparably shorter decay during second low field. This is due to the difference in the KWW-function during rise and decay, and can be attributed to the same timescale $\tau$. Further fitting parameters, apart from the timescale $\tau$, are the instantaneous response $A$ and the continuous response $B$, which are related to the response data as shown schematically in fig. 3.4. For all fits the value of the Kohlrausch-William-Watts-exponent is set to a constant value of $\beta_{K W W}=0.65$ according to the derivation from literature data for $\mathrm{Pd}_{40} \mathrm{Ni}_{40} \mathrm{P}_{20}$ metallic glass (cf. page 33).

\subsection{Incremental Analysis}

The incremental analysis connects the experimental data to avalanche dynamics theory. It aims on the identification of piece-wise linear behavior interrupted by breakdowns of the elasticity according to the EIP-motif or avalanches resulting from cooperative behavior of STZ $\beta$ as discussed in theory section 2.7.2. The definition of intervals of strain, and the connection to interval stress, interval duration, and apparent interval compliance is given in detail. A description of the extraction of average values and distributions on the interval strain concludes this section.

The excitation signal imposed on the DMA is assumed to result in a sinusoidal force working on the sample. However, the true force acting on the sample is not measured, stress data used during the analysis is due to the 'ideal' sinusoidal signal applied to the DMA. Thus, the strain signal is expected to carry the information on avalanche dynamics. The strain data provides 1000 data points per period and 3600 periods for a given stress amplitude in either initial low field or high field. The data collected during second low field are not evaluated by the incremental approach. Due to the excess of data, the incremental analysis is confined to the positive ascending part of the sinusoidal signal for each period. The data for the remaining three quarters of each period is still open for evaluation. The incremental analysis was performed by several MatLab routines, which are presented in detail in the appendix, section E. 


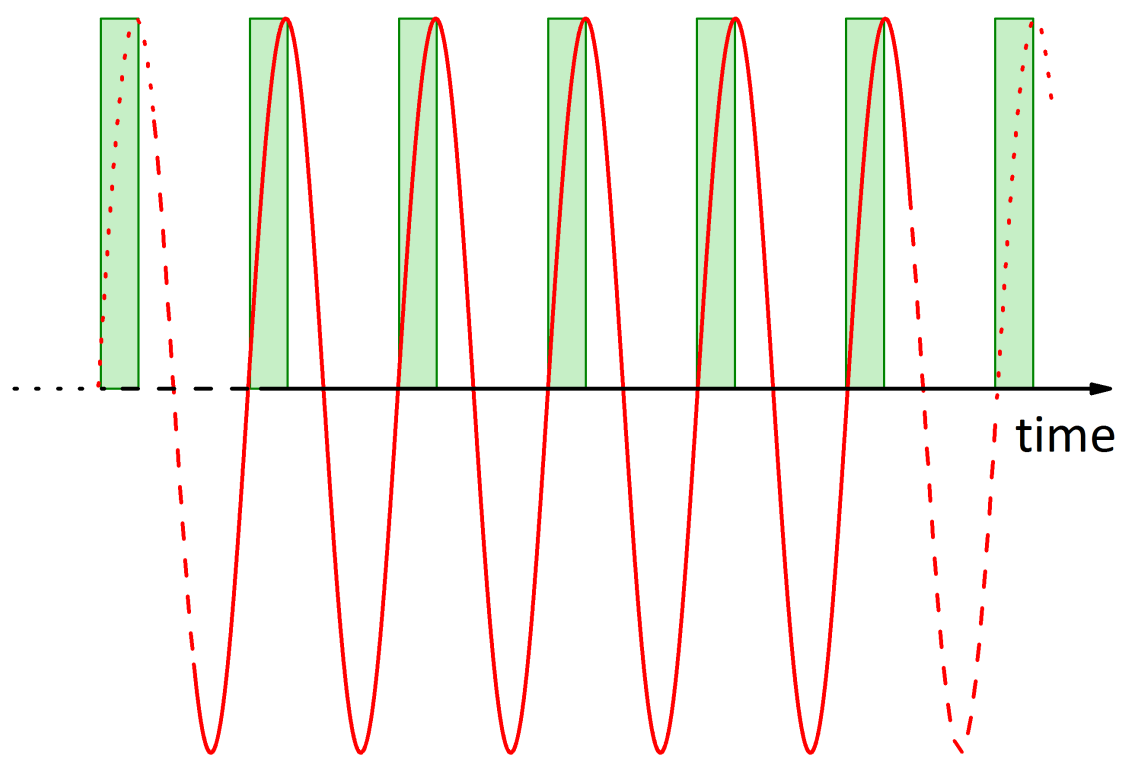

Figure 3.5 Scheme of oscillating excitation or response with positive ascending parts of each period marked by green rectangles.

\subsubsection{Extraction of Positive Ascending Part of each Period}

To identify the positive ascending parts of the response signal, the strain data is fitted by a sine over two successive periods, shifted period by period. From each fit the zero transitions, as well as the minimum and maximum of the first period are deduced and used to separate each period into four branches. These are the positive ascending, the positive descending, the negative descending and the negative ascending branch. Each branch consists of 250 data points. Zero crossings and extrema are deduced from the strain data. The stress data is period-wise categorized based on the strain branches, even though a phase shift occurs connected to the arising loss in the experiments. Thus, the positive ascending branch extracted for the strain will always start at the zero crossing and end in the maximum strain of that period due to the definition of the protocol of evaluation. The according stress branch might start at stresses larger than zero and end at a stress lower than the maximum stress, as the maximum position was already passed. An example of this correlation and the resulting strain-stress-curve is plotted in fig. 3.6. The data shown is the positive ascending part of an arbitrary period of the initial low field. As both strain and stress are of sinusoidal form, the data points in the strain-stress-curve are not equally distanced, but show a higher density of data points towards higher strain and stress values. The green dotted line separates the data into two halfs containing equal numbers of data points.

\subsubsection{Definition of Increments and Intervals}

The basis of the incremental analysis is the incremental stress and strain data, $\Delta \sigma_{\text {incr }}$ and $\Delta \epsilon_{i n c r}$, which are defined as the difference between two successive data points $m$ and $m+1$ :

$$
\begin{gathered}
\Delta \sigma_{i n c r}=\sigma_{m+1}-\sigma_{m} \quad \text { with } m \in[1: 249] \\
\Delta \epsilon_{i n c r}=\epsilon_{m+1}-\epsilon_{m} \quad \text { with } m \in[1: 249]
\end{gathered}
$$

As described in the theory section (see page 28), the material's response is assumed to be related to avalanche dynamics based on the EIP-motif in fig. 2.31. Thus, the incremental 


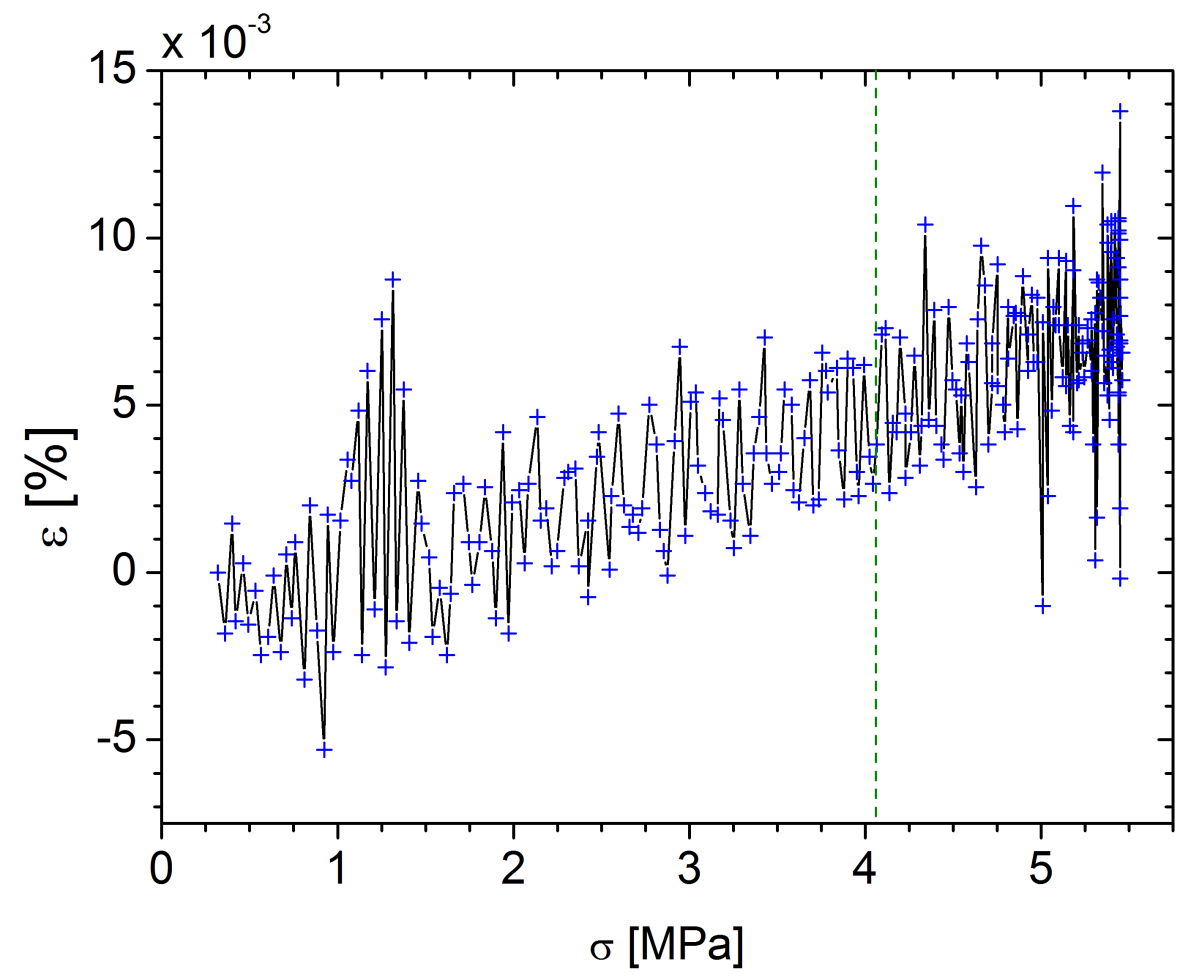

Figure 3.6 An example for a positive ascending branch of strain data, and the resulting plot of strain $\varepsilon$ against intra-periodic stress $\sigma_{c}$. The green dotted line separates the number of data points into two halfs of same number of data points.

stress and strain data are used to define intervals of stress and strain. An interval of strain $\Delta \epsilon_{i t v l}$ is defined as the sum of consecutive incremental strain steps $\Delta \epsilon_{i n c r}$ of same sign:

$$
\begin{gathered}
\Delta \epsilon_{i t v l}=\sum_{i n c r=A}^{B} \Delta \epsilon_{i n c r} \\
\Delta \sigma_{i t v l}=\sum_{i n c r=A}^{B} \Delta \sigma_{i n c r} \\
\Delta t_{i t v l}=B-A \\
\xi_{i t v l}=\Delta \epsilon_{i t v l} / \Delta \sigma_{i t v l}
\end{gathered}
$$

The interval stress $\Delta \sigma_{i t v l}$ is defined on the basis of initial and final data points $A$ and $B$ of correspondent strain intervals, and denotes the width of the interval in units of stress. This width of an interval can similarly be described in units of time by $\Delta t_{i t v l}$, which is referred to as the interval duration. To account for the dependence of the strain intervals on the exciting stress, a quantity in the units of a compliance can be defined for each interval according to equation $3.27 \mathrm{~d}$.

To investigate the dependence of the interval data on the experimental parameters, which are temperature $T$, stress amplitude $\sigma_{0}$, and intra-periodic stress $\sigma_{c}$, averages and according standard deviations of the interval data are determined. In this approach the incremental analysis is restricted to the evaluation of periods regarded as being in steady state. Generally, a sufficient number of periods is necessary for the calculation of interval averages and distributions. For a time-sensitive evaluation only few periods should attribute to the averaging process. This is in contradiction and explains why the steady state evaluation is more reliable. The average is extracted from the number of periods regarded as being in steady state, which is $N_{s s}=3600$ for initial low field data and refers to the last 2400 periods of high field 


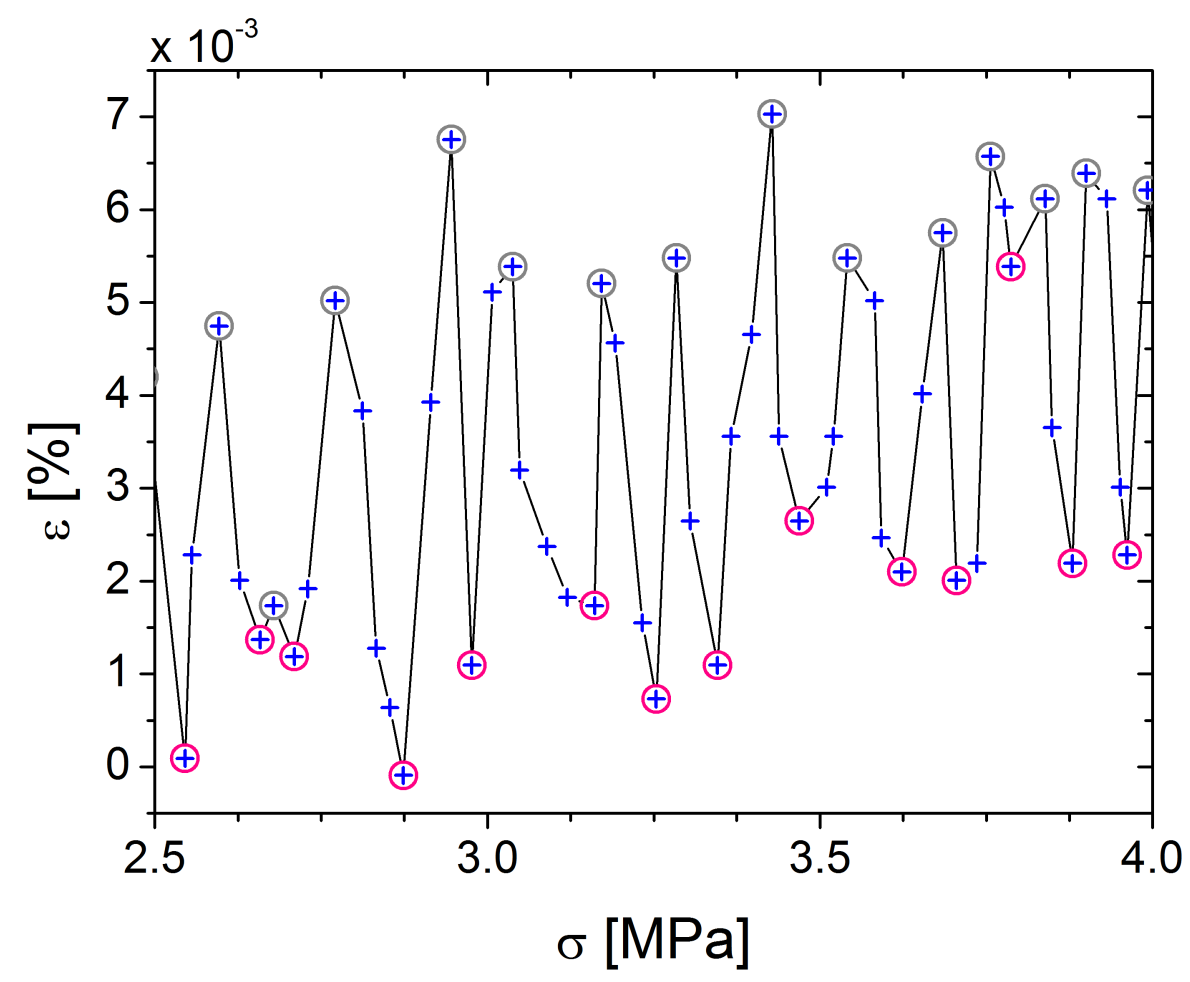

Figure 3.7 Zoom on data shown in fig. 3.6 Magenta circles depict the first data point belonging to an interval of increasing slope (further on called a "positive" interval), equal to the end of an interval with negative slope (referred to as a "negative" interval). Grey circles thus depict the first point of a negative and the final data point of a positive interval.

data. To distinguish between different levels of stress within the trajectory of a period, stress windows of $2 \mathrm{MPa}$ width are defined. These give a subset for averaging, corresponding to a binning with fixed window size. The stress of a binning window is termed as $\sigma_{c}$ and reflects the scale of the applied stress within a period. Thus it is referred to as the intra-periodic stress $\sigma_{c}$. The index declaring the stress window of interest is $c$, the number of intervals within a stress window $c$ of period $j$ is thus given by $n_{c}(j)$. This yields steady state mean values in dependence of $c$, e.g. in case of interval strain $\left\langle\left(\Delta \epsilon_{i t v l}\right)_{i j}^{L}\right\rangle_{c}$. These are extracted separately for low field and high field and either on intervals with positive strain $(\iota=P)$ or negative strain $(\iota=N)$ :

$$
<\left(\Delta \epsilon_{i t v l}\right)_{i j}^{L}>_{c}=\frac{\sum_{j=1}^{N_{s s}}\left(\left(\sum_{i=1}^{n_{c}(j)}\left(\Delta \epsilon_{i t v l}\right)_{i j}^{L}\right) / n_{c}(j)\right)}{N_{s s}}
$$

This equation describes the determination of averages on interval stress, interval duration, and interval apparent compliance as well, if the interval strain $\Delta \epsilon_{i t v l}$ in eq. 3.28 is replaced by $\Delta \sigma_{i t v l}, \Delta t_{i t v l}$, or $\xi_{i t v l}$ respectively.

The number of intervals contributing in the averaging process is in the order of $10^{3}$ in case of the high field data, and in the order of $10^{4}$ regarding low field data. The reasons for the difference in the number of intervals are simple. The average for high field data is based on steady state periods, i.e. 2400 periods, while the low field excitation is assumed as being in steady state over the full number of periods. Thus for low field averages, 3600 periods contribute to the averaging procedure. Moreover, the width of the stress window is set to $2 \mathrm{MPa}$, i.e. a fixed value, and the low field stress amplitude is $20 \%$ of the high field excitation. Thus the number of stress windows is reduced by a factor of five compared to the high field data, and therefore the number of intervals per stress window is generally larger for low fields. 
As the strain rate $\dot{\varepsilon}$ plays an important role for the interpretation of the data, its dependence on temperature $T$, stress amplitude $\sigma_{0}$, and intra-periodic stress $\sigma_{c}$ is extracted. The sum of interval strains $\Delta \varepsilon_{i t v l}$ for positive and negative intervals over stress windows including 50 data points each is taken. This sum is divided by the related duration, i.e. $\Delta t=50 \mathrm{~ms}$.

$$
\dot{\varepsilon}_{x}=\frac{\sum_{j=1}^{N_{s s}}\left(\sum_{k=X}^{X+50}\left(\Delta \epsilon_{i t v l}\right)_{k}\right)}{N_{s s} \cdot \Delta t} \text { for } X=50 x, x \in[0 ; 1 ; 2 ; 3 ; 4]
$$

\subsubsection{Generation of Double-Logarithmic Distributions}

To generate distributions on a quantity $X$, as for example the strain intervals $\Delta \varepsilon_{i t v l}$, the probability of a value to occur is determined based on the number of counts of this value. In the case of a non-discrete quantity as $\Delta \varepsilon_{i t v}$, a binning is defined, which separates the range of occurring values into boxes. For each box the number of values belonging into the box is connected to the mean value of the parameter range of the box. As an example, if a quantity $X$ would yield values between 0 and 10 , with a binning of $N_{\text {bins }}=20$ a box would have a width of 0.5 . In the box ranging from 1.5 to 2.0 , all $X$ contribute which exhibit values between these two numbers, and the number of these elements is counted. The mean of the box range is 1.75 , which is the $x$-value of the box in the plot of the distribution, while the number of elements in the box is plotted on the y-axis.

To investigate power-law behavior, the distributions are plotted in double-logarithmic scaling to extract the slopes of linear regimes in this scaling by linear regression. These slopes $\lambda$ correspond to the exponents of the power-law behavior as:

$$
\begin{aligned}
\log (P(X)) & =\lambda \cdot \log (X) \\
& =\log \left(X^{\lambda}\right)
\end{aligned}
$$

This corresponds to a power-law behavior in linear scaling, which contains $\lambda$ as the exponent of the quantity of interest $X$.

$$
P(X)=X^{\lambda}
$$

To extract the slope by linear regression, an equidistant binning is required. Otherwise those regions of the fitting-range would be weighted which obtain a higher density of bins. Therefore, it is necessary to apply a logarithmic binning, which is equidistant on the log-scale, and which is accordingly not acquired on the linear scale. It is achieved by determination of the maximum and minimum of the data set, giving the full range of the linear $x$-scale of the distribution. On these values the logarithmic function is applied, yielding the full range of the logarithmic x-scale. A number of bins $N_{b i n s}$ is defined, which partitions this range into boxes of equal width in log-scale. To generate the distribution, the width of the boxes is transformed into linear scale and the data of interest is categorized into the boxes and the elements are counted. This way, a distribution in linear scaling is deduced based on boxes which are equally wide in log-space, but not in linear space. To account for the difference in width between the boxes, the number of counts of each box is normalized by division with the box's width. Finally, the data is transformed into logarithmic scaling by taking the logarithm to base 10. To plot the distribution, the normalized number of counts, i.e. the probability $P(X)$, is plotted against the mean value of the range of the boxes, which corresponds to a value of the quantity $X$, in double-logarithmic scaling. 


\section{Chapter 4}

\section{Sample Characterization}

Several methods aiming on sample characterization were executed to ensure that the prepared metallic glass samples meet the required properties. Therefore, their characteristics regarding the amorphous state, as well as the mechanical and calorimetric behavior were analyzed. The results are presented in form of XRD-spectra, the composition measured by EDX, the calorimetric and mechanically probed glass transition temperature $T_{g}$, the temperature of crystallization $T_{x}$, the storage and loss modulus, $E^{\prime}$ and $E^{\prime \prime}$, as well as the storage and loss compliance $\chi^{\prime}$ and $\chi^{\prime \prime}$.

\subsection{X-ray Diffractometry}

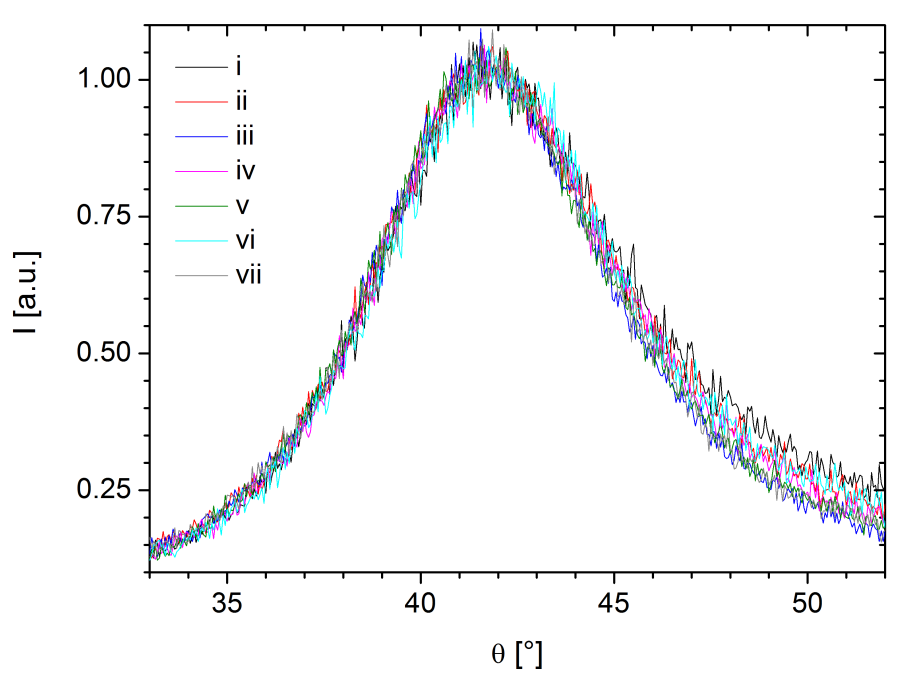

Figure 4.1 Normalized intensity I plotted against angle $\Theta$ for various samples. Samples $\mathrm{i}$ to v measured before, samples vi and vii measured after mechanical high-field experiments.

To ensure the amorphous state of the prepared metallic glass samples, each sample is measured by X-ray Diffractometry (XRD). As expected for glassy materials, the samples do not show crystalline peaks, but an amorphous halo which exhibits a maximum at $42.2^{\circ}$. In fig. 4.1 detailed XRD-scans of several samples both before and after mechanical treatment are shown. All scans presented here are performed on the surfaces which were cut using the diamond saw as described before. As-cast and mechanically treated samples show perfectly amorphous spectra, giving evidence that the preparation technique yields samples in the amorphous state, which is not affected by LAOS-measurements in the sense of a promoted crystallization. 


\subsection{Energy Dispersive X-ray Spectroscopy}

Samples of different casts were analysed by EDX and the average quantification is determined to 39.7(4) \%at. for Palladium (Pd), 39.4(7) \% at. for Nickel (Ni), and 20.9(9) \%at. for Phosphorous (P). Other elements are not detected with certainty, or could be excluded from the analysis as elaborated in section 3.3.

\subsection{Differential Scanning Calorimetry}

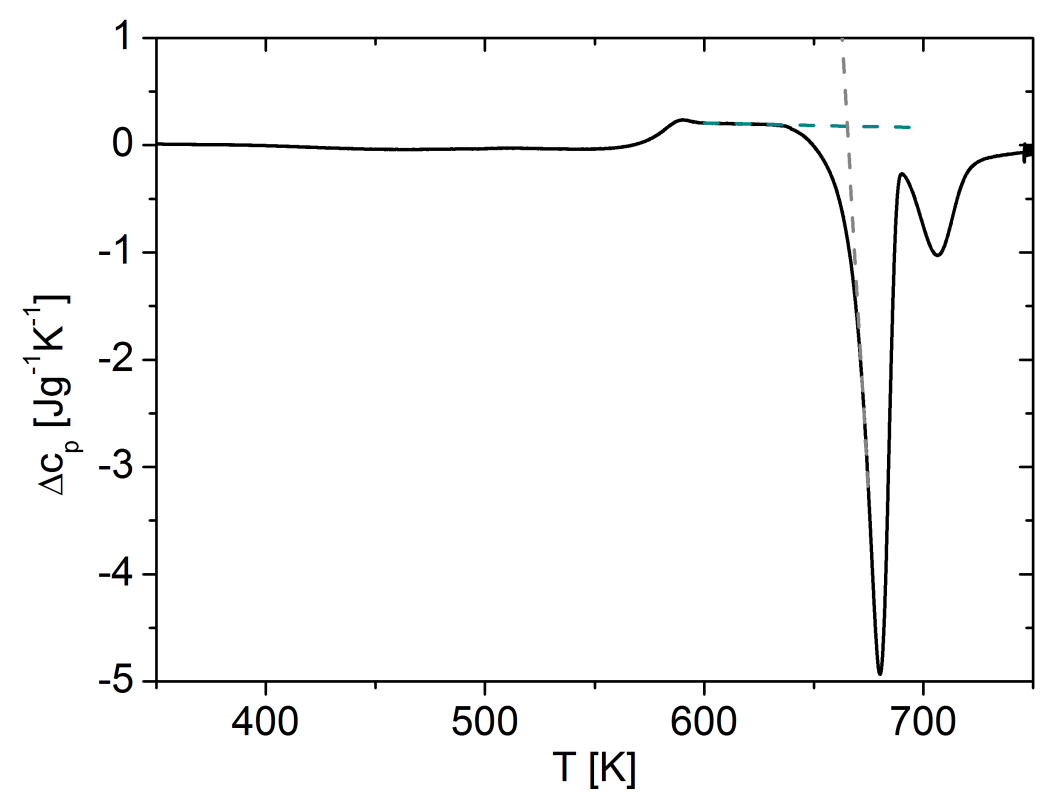

Figure 4.2 Plot of the specific heat contribution $\Delta c_{p}$ of the amorphous material against temperature $T$ derived from a DSC-measurement at a heating rate of $10 \mathrm{~K} \mathrm{~min}^{-1}$. Magenta and orange lines indicate linear fits to determine the onset of crystallization $T_{x}$ by the double-tangential method.

To determine the glass transition temperature, the onset of crystallization, and the specific heat of the material, differential scanning calorimetry (DSC)-scans are measured at a heating rate of $10 \mathrm{~K} \mathrm{~min}^{-1}$. In fig. 4.2 the change in specific heat $\Delta c_{p}$ is plotted against temperature $T$, and a calorimetric response typical for an amorphous material is observed. The values of $\Delta c_{p}$ correspond to the difference in specific heat between the glassy and the crystalline material (cf. sec. 3.4).

In the temperature regime of the glass up to $550 \mathrm{~K}, \Delta c_{p}$ shows a plateau of negligible difference between crystalline and amorphous specific heat. Fig. 4.3 shows a more detailed plot of the glass transition regime and the crystallization of the material. It becomes evident, that the plateau just before the glass transition regime shows a slight dip. At a temperature of roughly $570 \mathrm{~K}$ the glass transition sets fully in, the specific heat increases rapidly. It is terminated by an overshoot, which is heating rate dependent in general. It is followed by the regime of the supercooled liquid between $600 \mathrm{~K}$ and $630 \mathrm{~K}$. At a temperature of $665 \mathrm{~K}$ the onset of first crystallization is marked by an apparent decrease in specific heat which is technically due to the release of latent heat $\Delta H_{x}$ during crystallization. It is followed by a second stage crystallization at a temperature of $690 \mathrm{~K}$. After crystallization, the specific heat is at a level with the value observed in the glassy state of the sample, which is lower than in the supercooled liquid as shown in fig. 4.3. To give an average for the glass transition temperature, the mean value from six different DSC-measurements is calculated to $T_{g, D S C}^{\text {on }}=570(1) \mathrm{K}$. On a similar basis a mean value for the onset of first crystallization is determined to $T_{x 1}=665(4) \mathrm{K}$. 


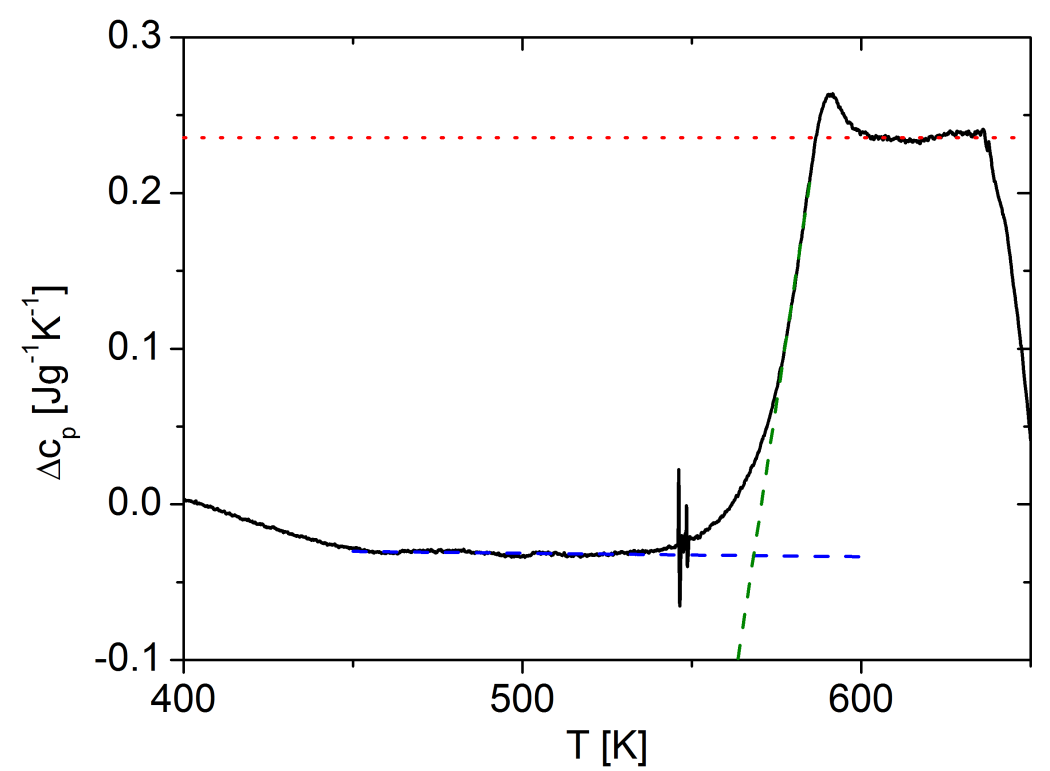

Figure 4.3 Plot of specific heat contribution $\Delta c_{p}$ of the amorphous material against temperature $T$ in the regime of the glass transition derived from a DSC-measurement with a heating rate of $10 \mathrm{~K} \mathrm{~min}^{-1}$. Dolid lines correspond to linear fits to determine the glass transition temperature of the sample by the double tangential method. The red dotted line indicates the level of specific heat of the supercooled liquid, from which $\Delta c_{p}^{s-1}$ is determined to $236(2) \mathrm{mJ} \mathrm{g}^{-1} \mathrm{~K}^{-1}$.

\subsection{Static Stress-Strain-Curves}

In order to gain a qualitative estimate of the nonlinearity of the mechanical sample response the relation of static stress to static strain is measured at $553 \mathrm{~K}$ and $563 \mathrm{~K}$ and plotted in fig. 4.4. The black data corresponds to the lower temperature of $553 \mathrm{~K}$ and exhibits an increasing behavior for stresses up to $170 \mathrm{MPa}$ corresponding to strains up to $1.3 \%$. The higher the strain, the less steep is the evolution of the stress-strain-curve until it reaches its maximum stress at $170 \mathrm{MPa}$ and a static strain of $1.3 \%$. Then the stress decreases with further increasing strain, initially rather shallow but then more abrupt down to a value of $155 \mathrm{MPa}$ when the experiment is terminated at $1.5 \%$.

The stress-strain-curve measured at $563 \mathrm{~K}$ shows a more compliant behavior compared to the data set measured at $553 \mathrm{~K}$, as the resulting strain is gained by a much lower stress than at lower temperature. Here, the stress increases in a more and more shallow fashion until it achieves a maximum value of $70 \mathrm{MPa}$ at a strain of $0.9 \%$. Then the stress decreases towards a value of $40 \mathrm{MPa}$ at a maximum strain of $1.5 \%$, while the slope gets steeper with increasing strain.

At both temperatures a regime is absent which is clearly identified as linear-like. The data curves are rather bend, i.e. exhibiting nonlinear behavior. If a linear regression of the initial part of the stress-strain-curve is fitted to the data, the slope yields a value of 41(1) GPa in case of the measurement at $553 \mathrm{~K}$, and a slope of 27(1) GPa in case of the measurement at $563 \mathrm{~K}$. In detail, the stress-strain-curves have their origin at stress and strain values, which clearly deviate from zero, leading to an offset stress loading the sample in addition to the excitation due to the measurement protocol. The offset stress is about $10 \mathrm{MPa}$ for the lower temperature and at $14 \mathrm{MPa}$ for the measurement at $563 \mathrm{~K}$. 


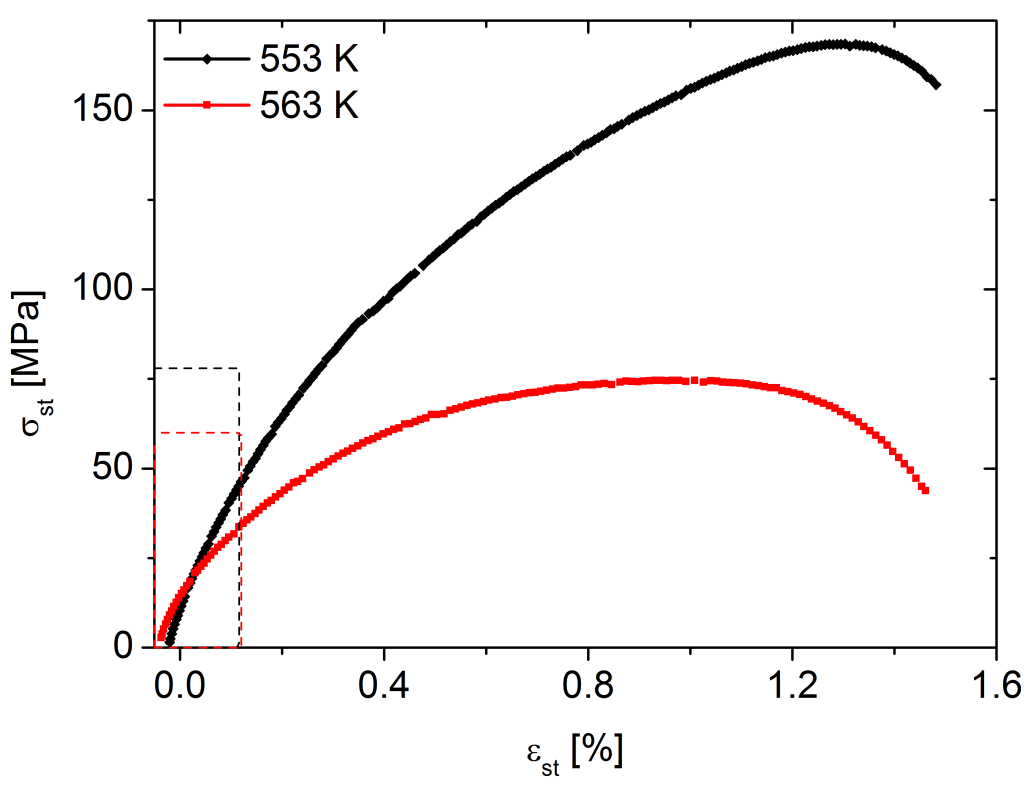

Figure 4.4 Static stress $\sigma_{s t}$ plotted against the static strain $\varepsilon_{s t}$ measured at two different temperatures. Dashed boxes indicate stress and strain regimes of LAOS-measurements.

\subsection{Dynamical Mechanical Temperature Scans}

In fig. 4.5 the storage and loss moduli $E^{\prime}$ and $E^{\prime \prime}$ are plotted against temperature for three arbitrary samples.

The storage modulus $E^{\prime}$ shows a linearly decreasing behavior for low temperatures. Absolute values for different samples exhibit values ranging between $60 \mathrm{GPa}$ and $70 \mathrm{GPa}$, resulting in a mean elastic modulus of $66(7) \mathrm{GPa}$ with an uncertainty of $15 \%$ relatively to the mean value, which is based on values extracted at a temperature of $320 \mathrm{~K}$. With eq. 2.17 this yields a shear modulus $G$ of 24 (3) GPa.

In the regime from $550 \mathrm{~K}$ to $625 \mathrm{~K}$ the glass transition region is clearly observable due to the sudden drop in storage modulus $E^{\prime}$ as expected due to Kramers-Kronig-relations. By evaluation with the double-tangential method the onset of the glass transition can be determined as an average over several measurements to $T_{g}^{\text {on }}=575(2) \mathrm{K}$.

The loss modulus $E^{\prime \prime}$ shows coherent behavior to $E^{\prime}$. While $E^{\prime \prime}$ is below the limit of resolution at low temperatures, measurable contributions evolve around $500 \mathrm{~K}$. At around $525 \mathrm{~K}$ a shoulder or excess wing is visible, which is typical for the $\beta$-relaxation. It is followed at $590 \mathrm{~K}$ by a maximum corresponding to primary or $\alpha$-relaxations. The position of the $\alpha$-relaxation coincides with the inflection point of the storage modulus $E^{\prime}$ at $T_{g}^{P}=598(2) \mathrm{K}$.

At temperatures above $630 \mathrm{~K}$ the increase in storage and loss modulus indicates the onset of crystallization. The affected temperature regime is marked by a red background.

Figure 4.6 shows the data of the modulus measurement transformed into mechanical storage and loss compliance, $\chi^{\prime}$ and $\chi^{\prime \prime}$. As the mechanical excitation during the temperature scan is very low, Hookean behavior is assumed to determine the compliance according to eq. 3.12 Even though the data contains identical information about the relation between storage and loss, the glass transition temperature, and the onset of crystallization, it gives insight into the compliance behavior, as measured in case of the LAOS-measurements. It becomes obvious that both the storage and the loss compliance increase with increasing temperature in agreement to literature data [125]. In the loss compliance, Newtonian flow dominates at temperatures above $T_{g}$ as Maxwell predicts (cf. eq. 2.13), which hides the loss peak in compliance. 


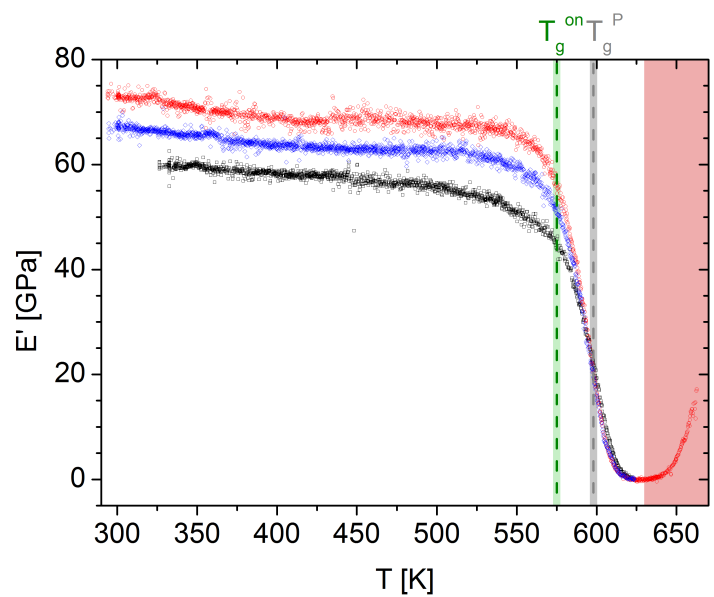

(a) Storage modulus $E^{\prime}$

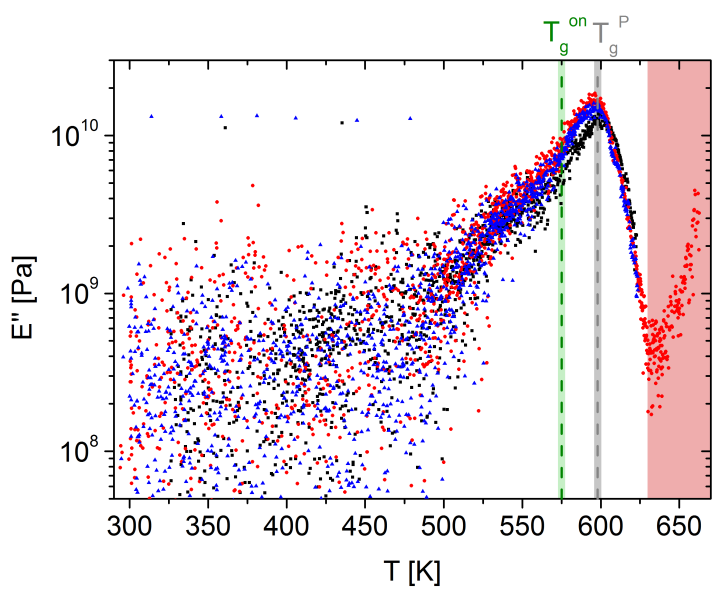

(b) Loss modulus $E^{\prime \prime}$

Figure 4.5 Storage modulus (a) and loss modulus (b), $E^{\prime}$ and $E^{\prime \prime}$, plotted versus temperature $T$ in a strain-controlled measurement at a displacement amplitude of $1 \mu \mathrm{m}$ and a scanning rate of $2 \mathrm{~K} \mathrm{~min}^{-1}$. The green and grey dashed lines depict the onset of the glass transition $T_{g}^{o n}$ and the loss-peak temperature $T_{g}^{P}$ with shaded areas in green and grey according to the standard deviation. The red-colored background corresponds to the section of the temperature scan in which the sample response is compromised by crystallization.

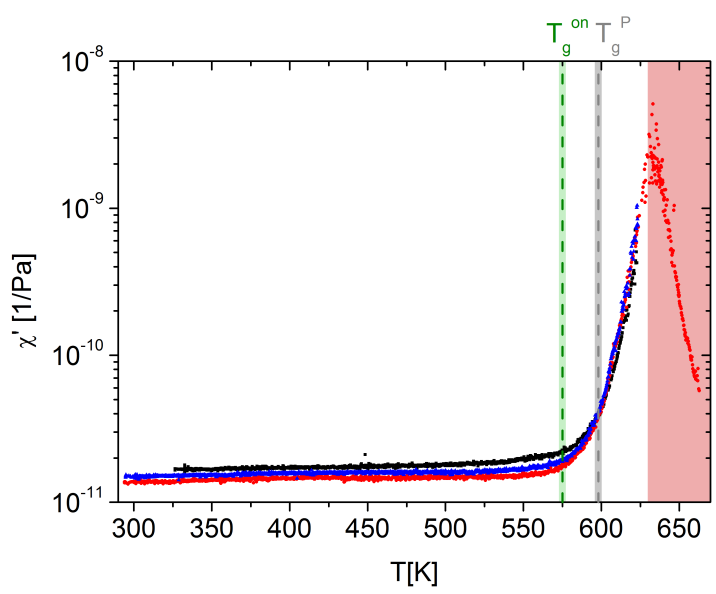

(a) Storage compliance $\chi^{\prime}$

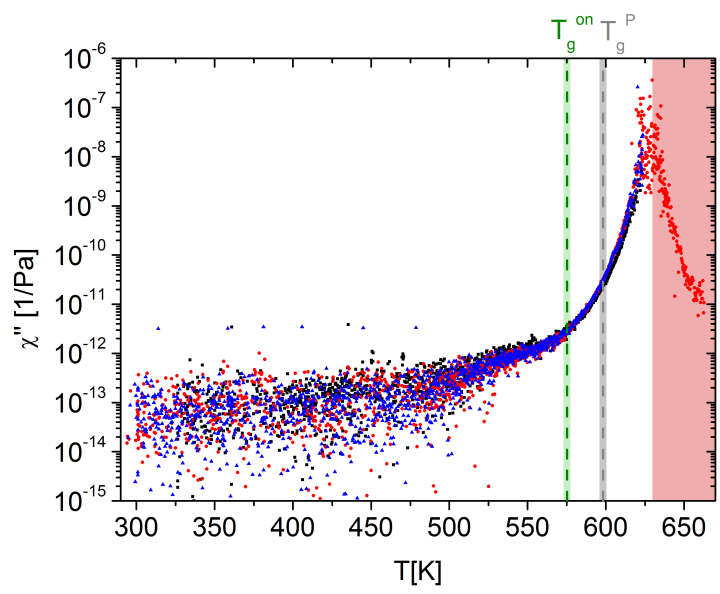

(b) Loss compliance $\chi^{\prime \prime}$

Figure 4.6 Storage compliance (a) and loss compliance (b), $\chi^{\prime}$ and $\chi^{\prime \prime}$, plotted versus temperature $T$, determined according to eq. 3.12 from the modulus due to low field temperature scans (cf. fig.4.5). The green and grey dashed lines depict the onset of the glass transition $T_{g}^{o n}$ and the loss-peak temperature $T_{g}^{P}$ with shaded areas in green and grey according to the standard deviation. The redcolored background corresponds to the section of the temperature scan in which the sample response is compromised by crystallization. 


\section{Chapter 5}

\section{Results on LAOS Measurements}

As described in section 3.5.2, a LAOS stress-protocol is applied to study the behavior of the metallic glass $\mathrm{Pd}_{40} \mathrm{Ni}_{40} \mathrm{P}_{20}$ in single cantilever bending geometry under high stress.

In the following section, qualitative results are presented in form of Lissajous-plots, containing the trajectories of stress and strain similar to the information given in a stress-strain-curve. The influence of stress amplitude $\sigma_{0}$ and temperature $T$ on the form of the Lissajous-plots is described.

Further on, the Fourier-analysis of the data is presented, based on the assumption of a globally nonlinear description of the compliance 3.6. The evolution of nonlinear contributions evolving with high stress amplitudes $\sigma_{0}$ is presented at the fundamental and third harmonic frequency. Finally, the incremental analysis is introduced which is based on the assumption of avalanche dynamics as elaborated in chapter 2.7.2. Averages and distributions of strain intervals are analyzed and the power-law behavior is evaluated in case of double-logarithmic distributions. 


\subsection{Lissajous-Plots}

To characterize the mechanical behavior of a material, stress-strain-curves are measured giving information on the response behavior to a mechanical excitation. Similar to stressstrain-curves, Lissajous-plots characterize the mechanical behavior in case of dynamical experiments. Therefore, the strain response $\hat{\varepsilon}$ is plotted against the exciting stress $\hat{\sigma}$ for one period, i.e. one sine wave of the LAOS experiments described in chapter 3.5.2. If not stated differently, the depicted period corresponds to the next to the last period measured at the specified stress amplitude and is thus assumed to be in steady state.

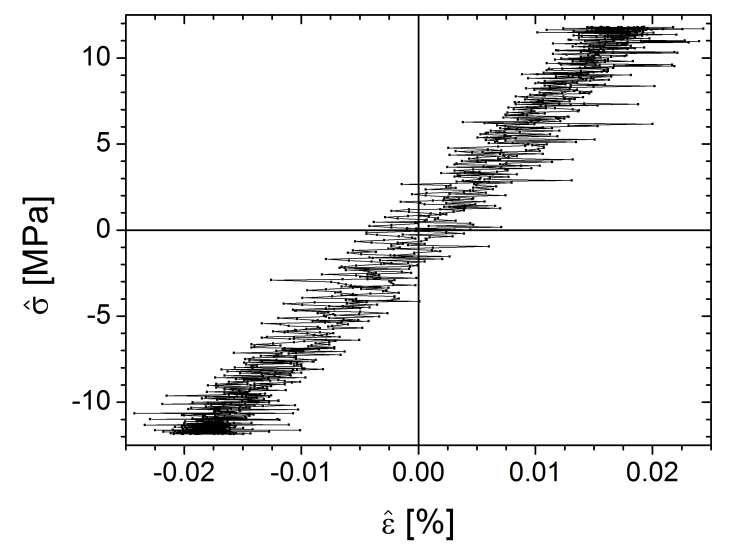

(a) $\sigma_{0}=12 \mathrm{MPa}$

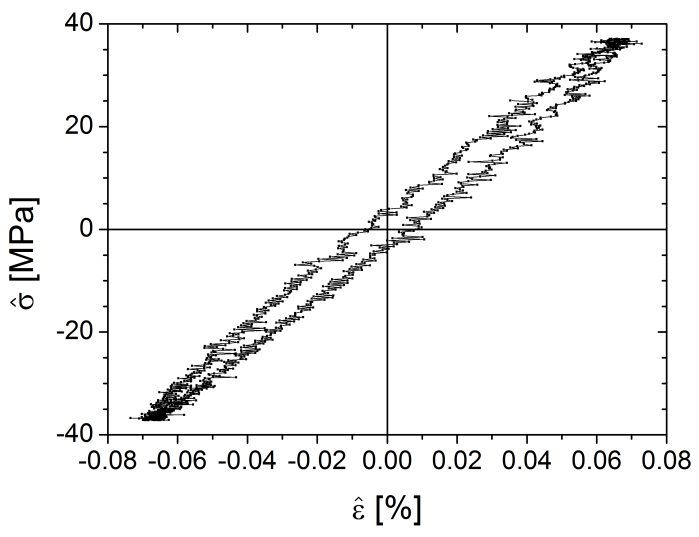

(c) $\sigma_{0}=37 \mathrm{MPa}$

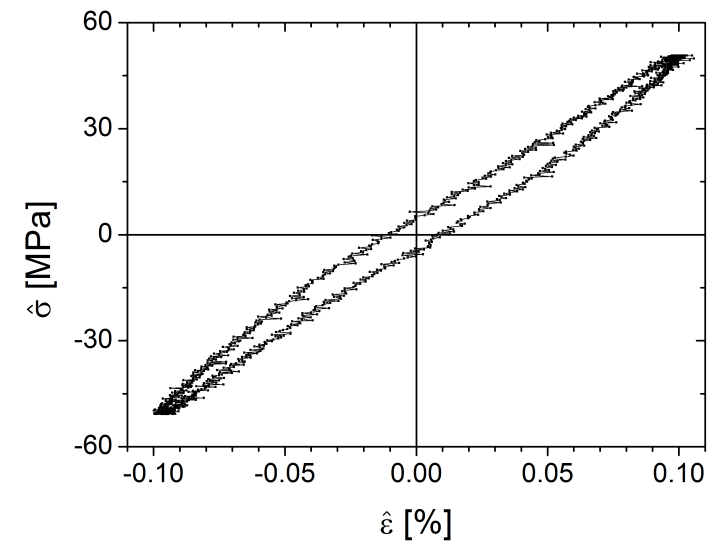

(e) $\sigma_{0}=51 \mathrm{MPa}$

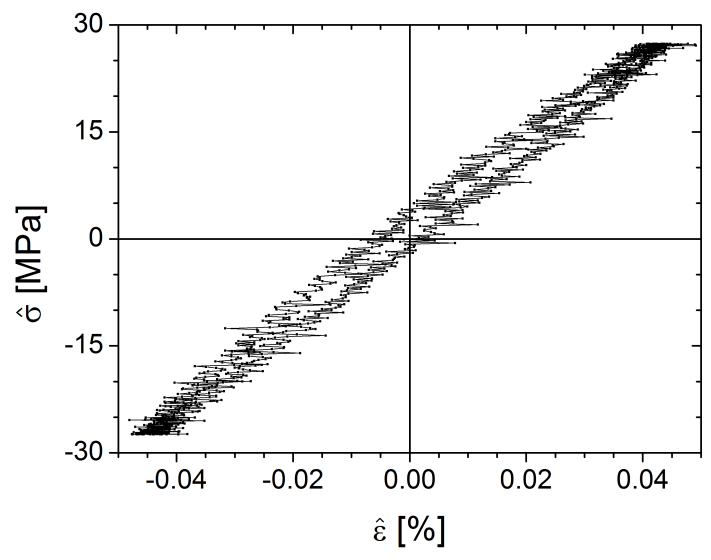

(b) $\sigma_{0}=26 \mathrm{MPa}$

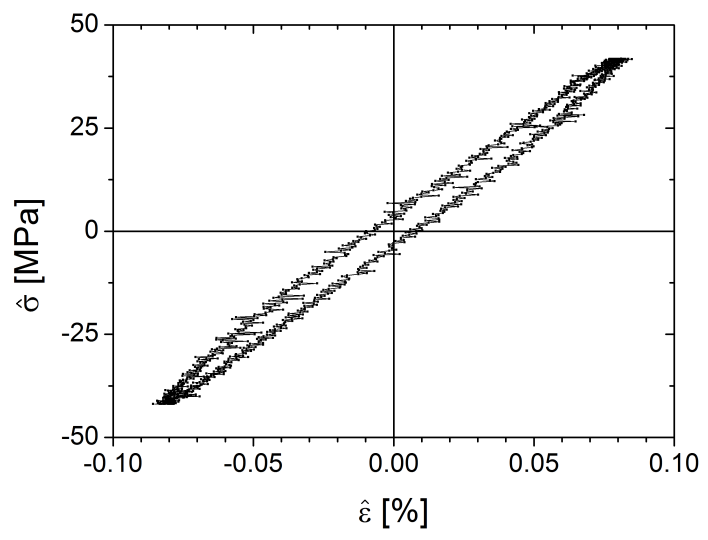

(d) $\sigma_{0}=42 \mathrm{MPa}$

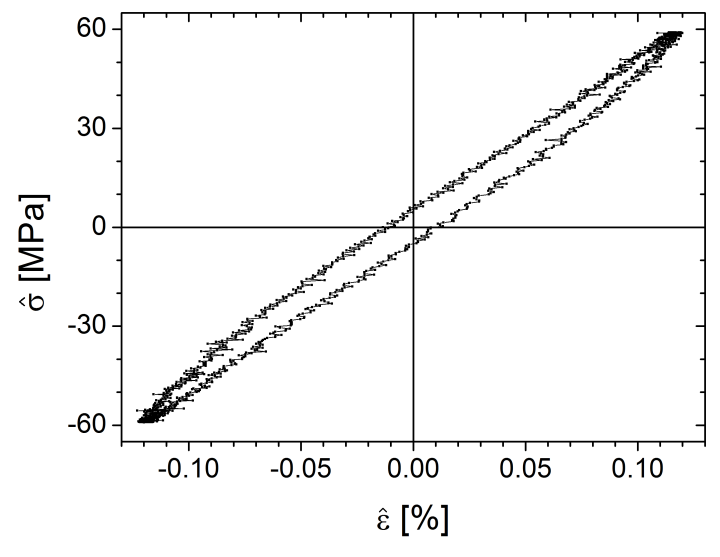

(f) $\sigma_{0}=59 \mathrm{MPa}$

Figure 5.1 Lissajous-plots of stress $\hat{\sigma}$ and strain $\hat{\epsilon}$ for increasing stress amplitude $\sigma_{0}$ at a temperature of $563 \mathrm{~K}$. 


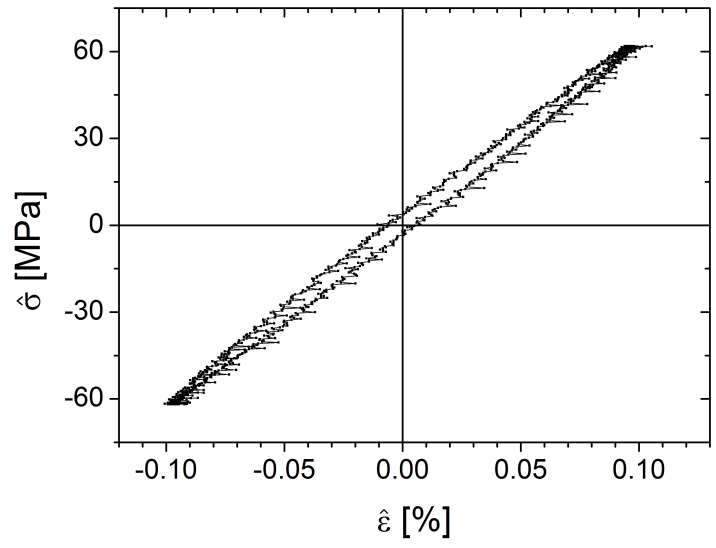

(a) $T=553 \mathrm{~K}$

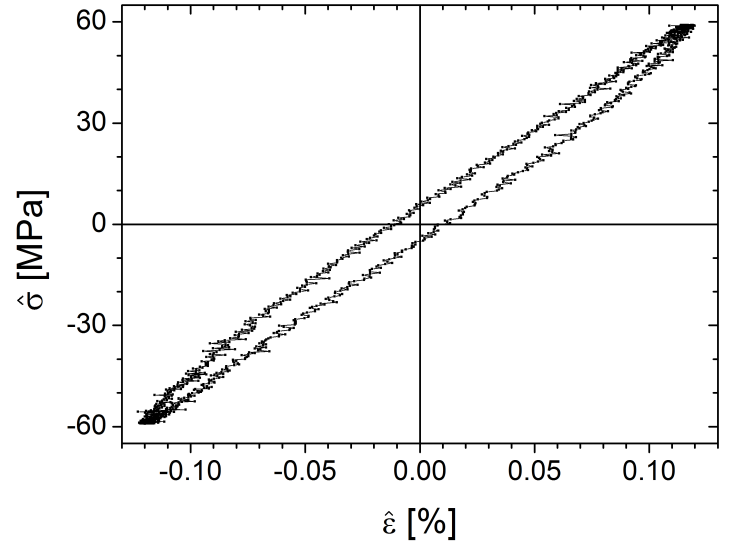

(b) $T=563 \mathrm{~K}$

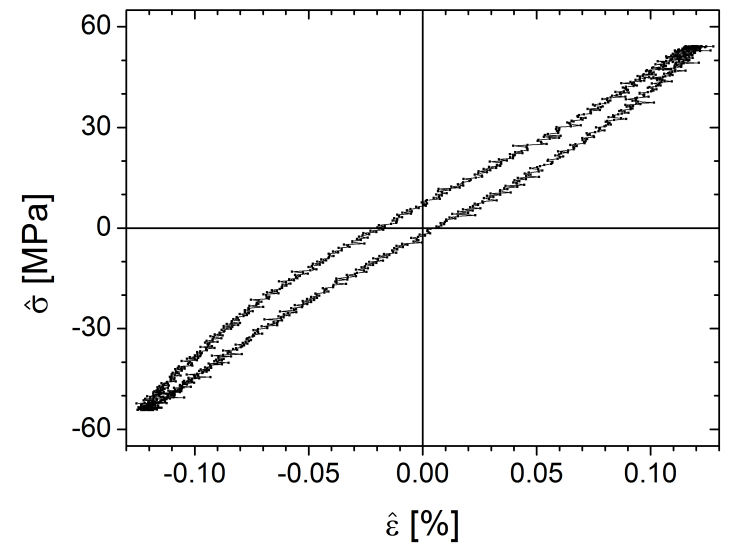

(c) $T=565 \mathrm{~K}$

Figure 5.2 Lissajous-plots of stress $\hat{\sigma}$ and strain $\hat{\epsilon}$ for increasing temperature at a stress amplitude $\sigma_{0}$ of about $60 \mathrm{MPa}$.

Fig. 5.1 shows Lissajous-plots of measurements at a temperature of $563 \mathrm{~K}$ at six different stress amplitudes. Qualitative changes in the form of the plot under increasing stress amplitude are observed. In fig. 5.1a the response behavior in strain to a stress amplitude of $12 \mathrm{MPa}$ resembles a straight line, as there is no significant difference between the branch of increasing and the branch of decreasing stress values. The maximum strain is rather small with a value of $0.02 \%$. Plots in fig. $5.1 \mathrm{~b}$ and fig. $5.1 \mathrm{c}$ show a more elliptic shape. The area within the ellipsis tends to increase with increasing sress amplitude $\sigma_{0}$. At a stress amplitude of $26 \mathrm{MPa}$ the maximum strain is $0.045 \%$, and it further increases with increasing stress amplitude. In fig. 5.1d to fig. 5.1f the form of the data resembles a distorted ellipsis, as the slope in the regime of small stress values is tilting towards lower numbers. The slope at high stresses in contrast remains at the value observed for the data at low stress amplitude, e.g. as in fig. 5.1a. Stress amplitudes range from $42 \mathrm{MPa}$ to $59 \mathrm{MPa}$ with a maximum response in strain of $0.10 \%$ to $0.12 \%$.

To give an impression how the form of the stress-strain-plots depends on the temperature $T$, fig. 5.2 gives an overview of measurements of comparable stress amplitude $\sigma_{0}$ at different temperatures. The temperature influences the slope of the stress-strain-curves as data at $553 \mathrm{~K}$ show a steeper stress-strain relation than data at $563 \mathrm{~K}$ and $565 \mathrm{~K}$. Moreover, fig. 5.2a shows an elliptical shape, whereas fig. $5.2 \mathrm{~b}$ indicates a tendency to a superimposed tilt in the regime of small stresses, which is even stronger in fig. [5.2C. A general observation is that the higher the temperature is, the more pronounced the difference in the slope in the regime of small stresses becomes relative to the slope at high stresses. 
The six plots in fig. 5.3 represent six different sine waves as six different points in time during high field excitation, namely periods number 1, 51, 101, 1201, 2401, and 3598.

Fig. 5.3a depicts the stress-strain-relation during the very first period of high field excitation, and it exhibits a rather elliptical shape. Fig. 5.3b shows the stress-strain-relation during the 51st period of high field excitation, fig. 5.3c the 101st period. Both plots show a steeper slope at high stresses than at low stresses. This feature evolves more and more with time, as can be seen in figures 5.3d to 5.3f. which represent data from periods number 1201,2401 , and 3598. The steepness of slope at high stresses is constant with time, while the value of the slope at low stresses decreases.

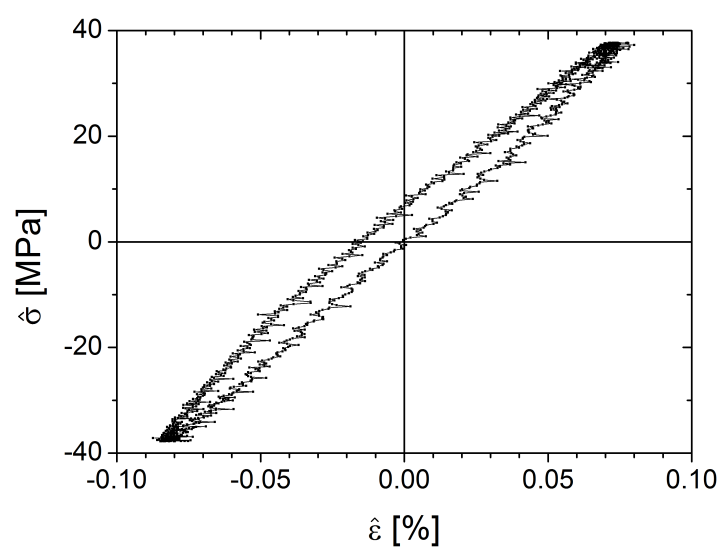

(a) 1 st period

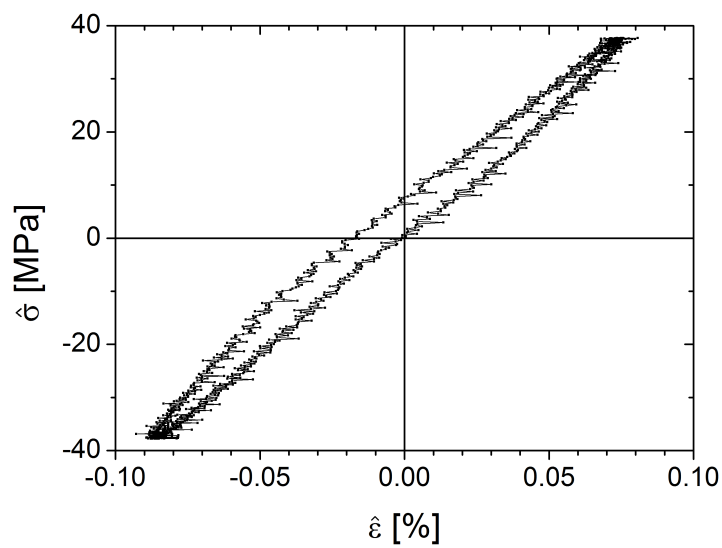

(c) $101 \mathrm{st}$ period

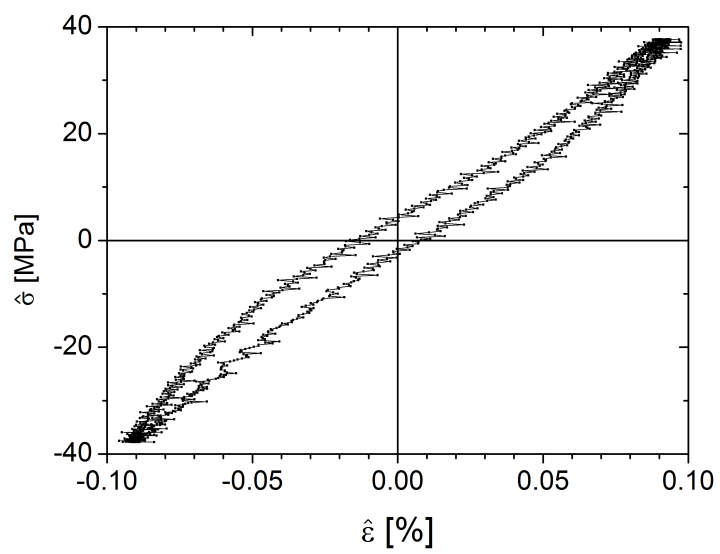

(e) 2401st period

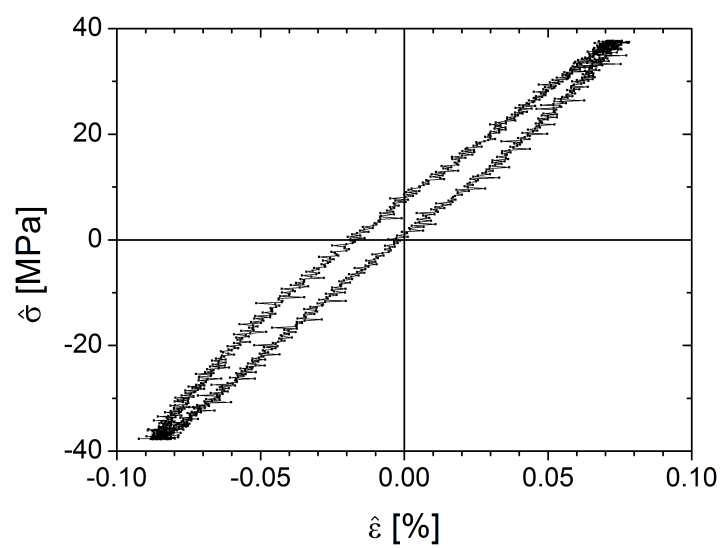

(b) 51 st period

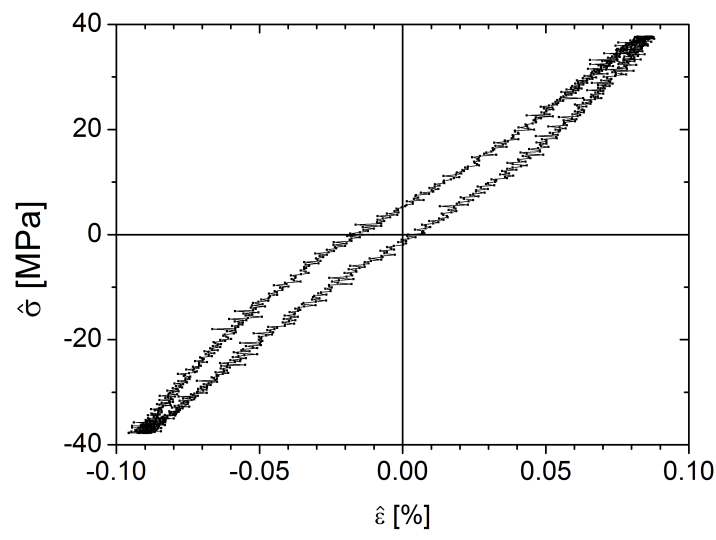

(d) 1201st period

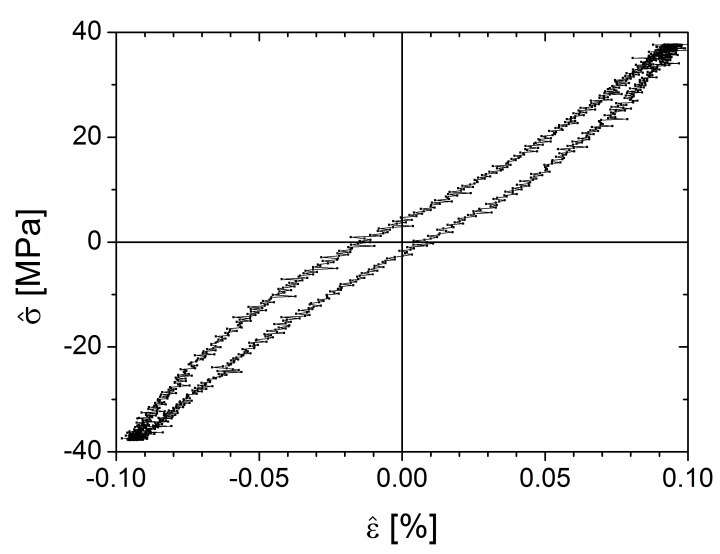

(f) Next to the last (3598th) period

Figure 5.3 Lissajous-plots of stress $\hat{\sigma}$ and strain $\hat{\epsilon}$ for periods with increasing experimental time during high field excitation at a temperature of $565 \mathrm{~K}$ and a stress amplitude of $37 \mathrm{MPa}$. 


\subsection{Comparison of LAOS-Measurements to Temperature Scans}

As the LAOS-experiments are stress controlled, the compliance characterizes the material's response. In contrast, the performed temperature scans measure the loss and storage modulus under very low strain amplitude. As the strain excitation is very small, these modulus values are transformed into compliance data according to equation 3.12. In fig. 5.4 a section of the temperature scans is shown and compared to LAOS-experiments. The two measurement-protocols, i.e. the temperature scans and the LAOS excitation yield compliance data in good agreement in case of LAOS-data from the initial low field (green symbols). Both storage and loss data extracted from LAOS high field (red symbols) yield higher values for the compliance, i.e. the material response exhibits super-linear behavior under high-field excitation.

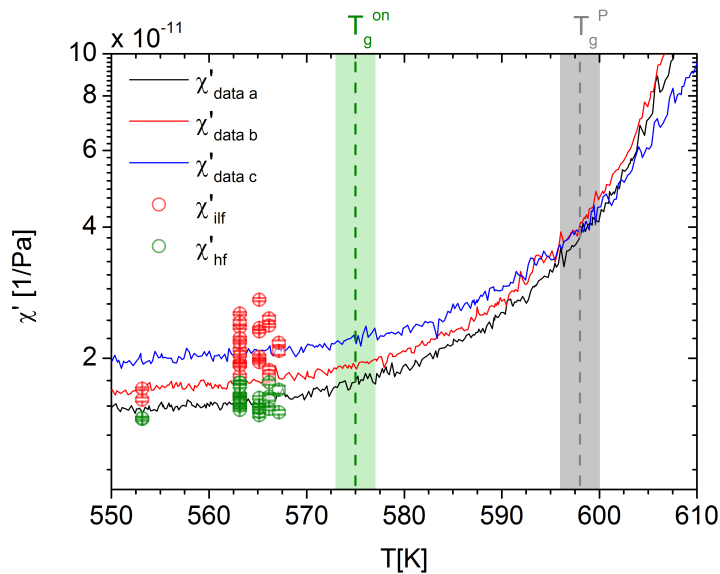

(a) Storage compliance $\chi^{\prime}$

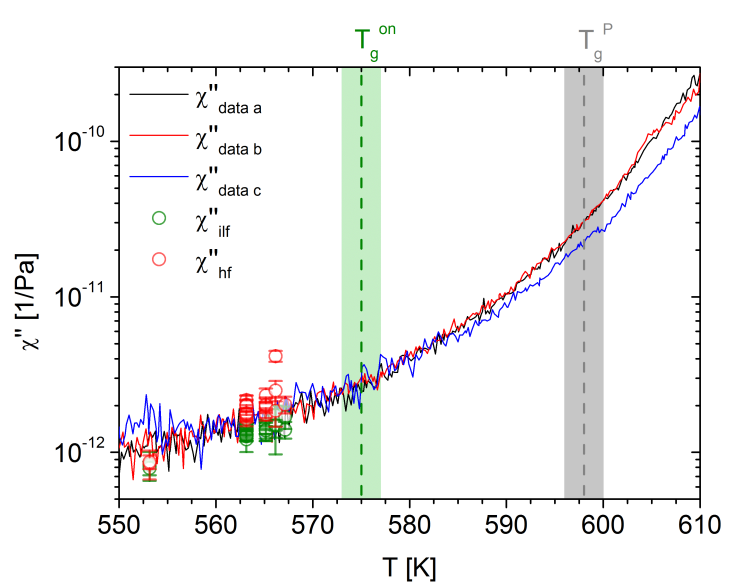

(b) Loss compliance $\chi^{\prime \prime}$

Figure 5.4 Comparison of temperature scans at low displacement amplitude (lines) and initial low field values from LAOS experiments (green symbols) for storage (a) and loss (b) compliance, $\chi^{\prime}$ and $\chi^{\prime \prime}$, plotted versus temperature $T$. Red symbols are steady state values under high field excitation extracted from LAOS experiments. Data from temperature scans corresponds to data shown in fig. 4.6 and $4.5 T_{g}^{o n}$ and $T_{g}^{P}$ correspond to the temperatures of the onset of the glass transition and of the loss peak position. 


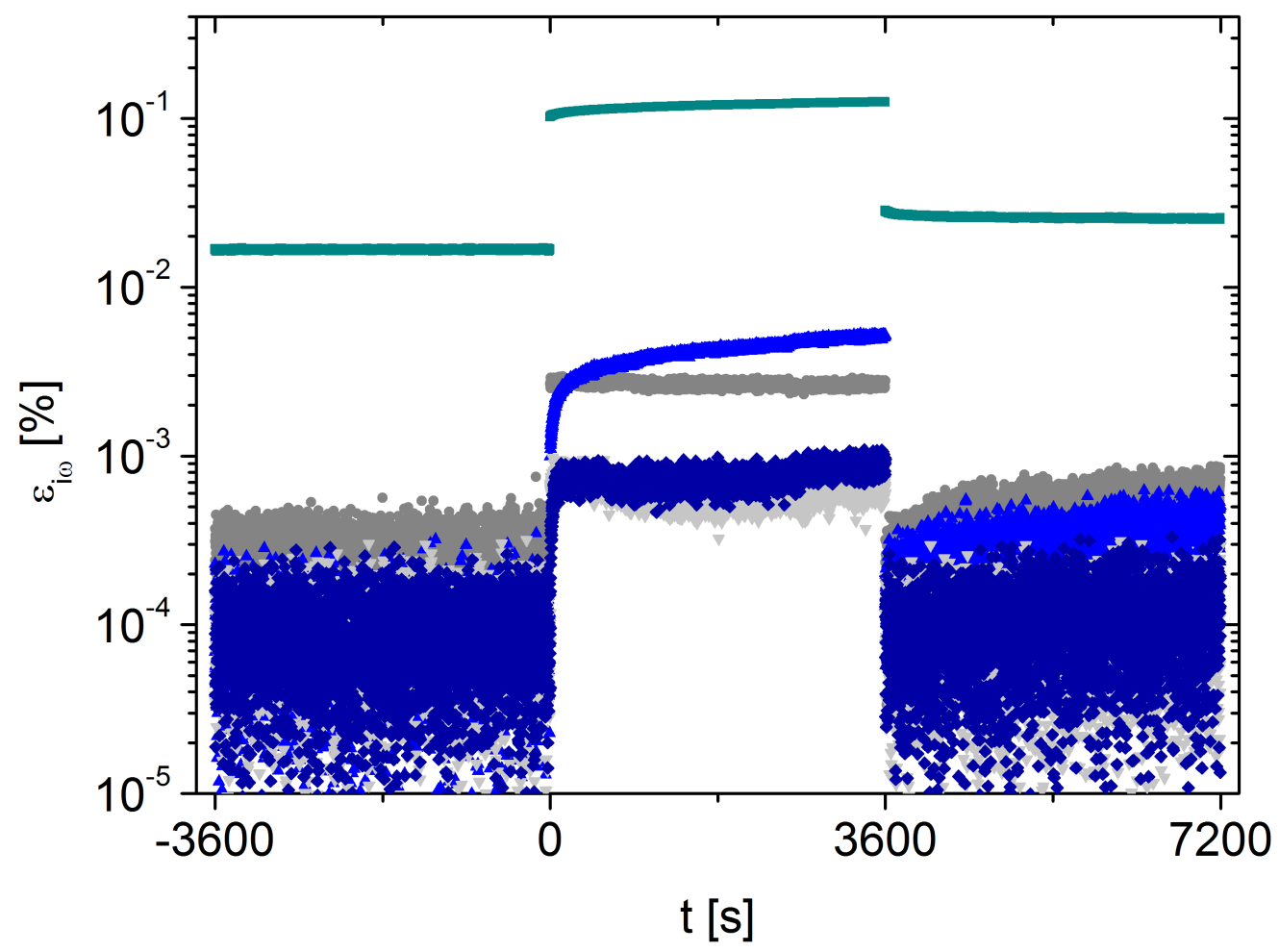

Figure 5.5 Strain amplitudes for $1^{\text {st }}(\square), 2^{\text {nd }}(\bullet), 3^{\text {rd }}(\nabla), 4^{\text {th }}(\Delta)$, and $5^{\text {th }}(\diamond)$ harmonic frequency extracted by Fourier-analysis from LAOS-measurements performed at $565 \mathrm{~K}$ and a high field stress amplitude of $54 \mathrm{MPa}$.

\subsection{Nonlinear Analysis}

To extract the nonlinear response behavior, the period data on exciting stress and resulting strain are period-by-period Fourier-analyzed to extract amplitudes and phase-shifts for fundamental and higher harmonic frequencies. The Fourier-amplitudes for the first to the fifth harmonic are plotted for a complete measurement in fig. 5.5 to give an example on the observed strain response. During initial low field excitation ( $-3600 \mathrm{~s}$ to $0 \mathrm{~s})$ only a contribution at the fundamental frequency is observed, the strain response is purely linear. During high field excitation ( $0 \mathrm{~s}$ to $3600 \mathrm{~s}$ ) nonlinear contributions appear, both at the fundamental and at higher order frequencies. First and third order contributions show a time-dependent evolution and strongest contributions, while even harmonic contributions are less intense than the signal at even harmonic frequency of higher order. During the second low field (3600 s to 7200 s) only a contribution at the fundamental frequency occurs, which ceases with time towards the value of initial low field. Further higher harmonic frequencies do not show significant contributions.

A quantitative analysis of the nonlinear contributions at fundamental and third harmonic frequency is presented. Both the storage and loss contribution of the nonlinear compliance at fundamental frequency is investigated regarding the nonlinear coefficients as well as the time-dependent behavior during high field and second low field. The complex compliance at third harmonic frequency is evaluated in detail regarding the nonlinear coefficient and the time-dependent rise during high field. 


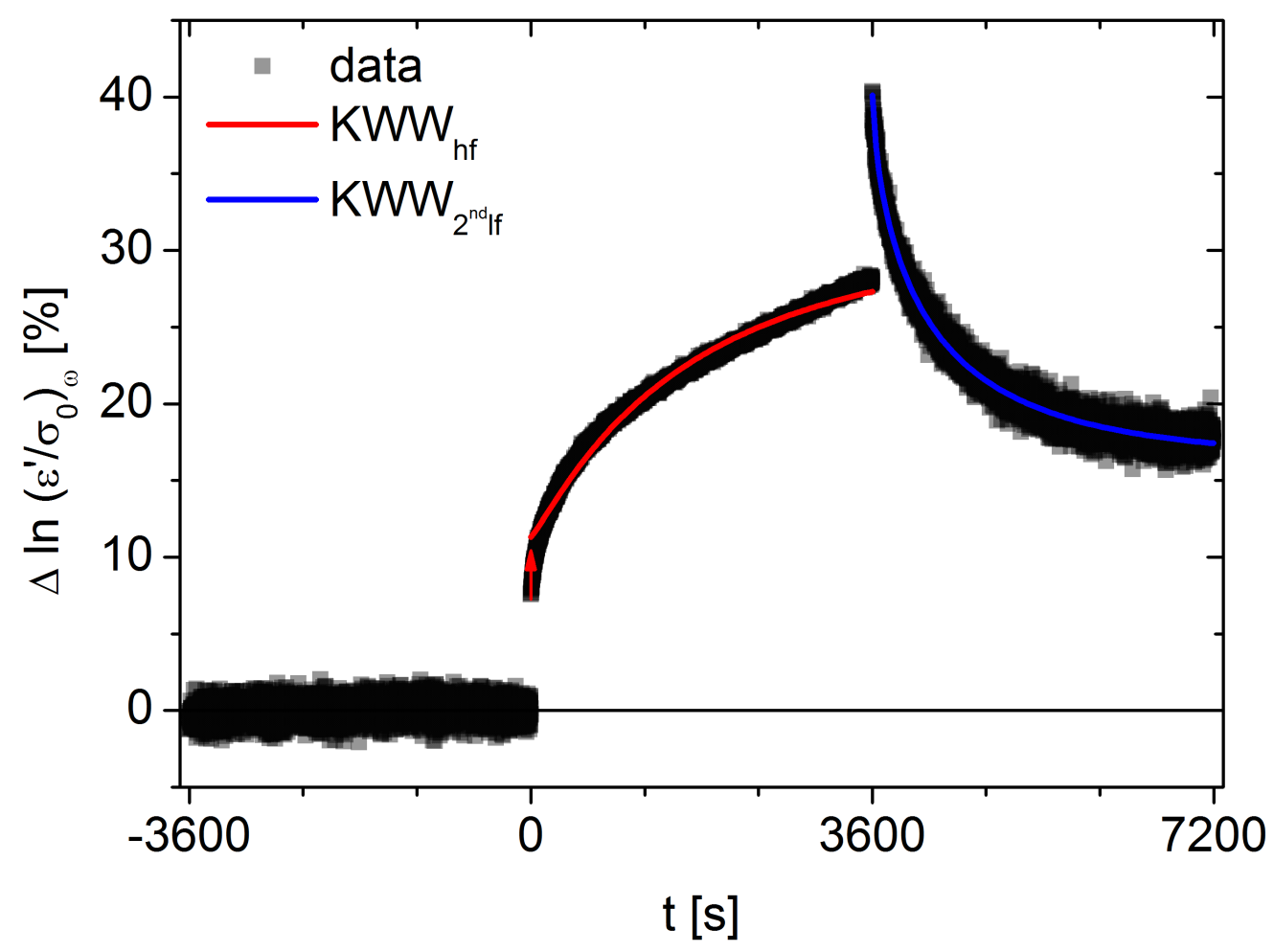

Figure 5.6 Example for time-dependent behavior of nonlinear relative change in storage compliance at fundamental frequency $\Delta \ln \left(\varepsilon^{\prime} / \sigma_{0}\right)_{\omega}$ at $T=563 \mathrm{~K}$ and $\sigma_{0}=51 \mathrm{MPa}$.

\subsubsection{Nonlinear Contribution of Storage Compliance at Fundamental Fre- quency during and after High Field Excitation}

For the analysis of nonlinear behavior due to high fields at elevated temperature, the nonlinear contribution at fundamental frequency $\Delta \ln \left(\varepsilon / \sigma_{0}\right)_{\omega}$ is of main interest. To extract this quantity, the compliance during the initial low field $\chi^{(1)}$ is subtracted from the overall compliance $\left(\varepsilon / \sigma_{0}\right)_{\omega}$ at fundamental frequency, and the resulting difference is divided by the initial low field value $\chi^{(1)}$ according to eq. 3.21 .

In this chapter, the nonlinear contribution is calculated for the storage compliance, denoted as $\Delta \ln \left(\varepsilon^{\prime} / \sigma_{0}\right)_{\omega}$. In fig 5.6 an example for the evolution of $\Delta \ln \left(\varepsilon^{\prime} / \sigma_{0}\right)_{\omega}$ with time is plotted. During the initial low field excitation a steady behavior is observed and the relative nonlinear contribution is negligible. When the excitation signal is switched to high field amplitudes, the storage compliance increases initially very rapidly, then the increase ceases. As soon as the excitation signal is switched back to low field amplitude, the signal instantaneously jumps to an initial value and decreases rapidly again, then approaches a low field plateau.

To evaluate the changes in $\Delta \ln \left(\varepsilon^{\prime} / \sigma_{0}\right)_{\omega}$ during and after high field excitation, squared KWW. functions are used for fitting as described in section 3.6.3. The quantities extracted are the timescale of the change in response signal, which is $\tau_{h f}$ or $\tau_{2^{\text {nd }} / f}$, the instantaneous change in signal $A_{K W W}$, and the continuous change in response $B_{K W W}$. The sum of instantaneous and continuous amplitude, $A_{K W W}+B_{K W W}$ yields the overall amplitude of the nonlinear behavior denoted as $\left[\Delta \ln \left(\varepsilon^{\prime} / \sigma_{0}\right)_{\omega}\right]_{h f}$ in case of evaluation of the high field behavior, and $\left[\Delta \ln \left(\varepsilon^{\prime} / \sigma_{0}\right)_{\omega}\right]_{2^{\text {nd }} / f}$ under second low field excitation. 


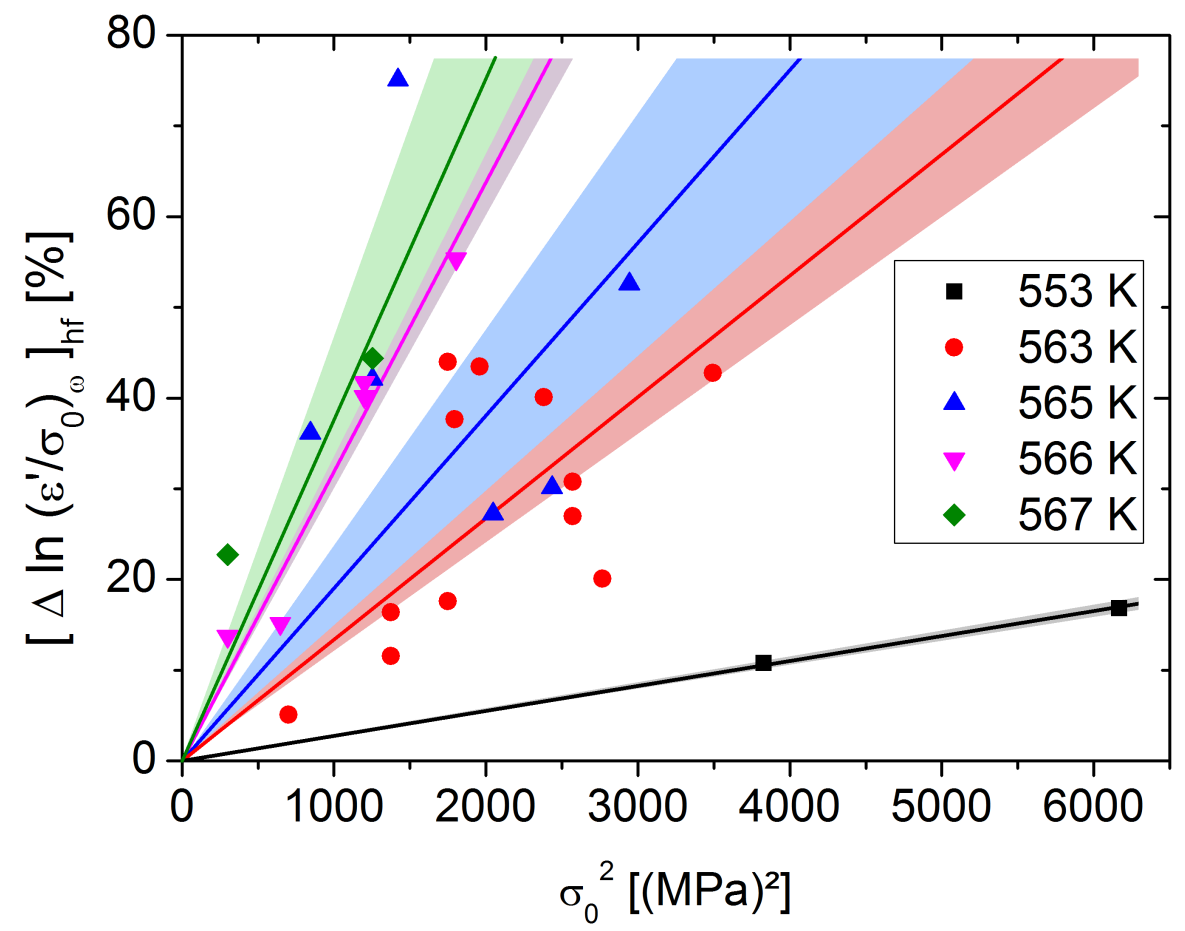

Figure 5.7 Steady state amplitude of relative nonlinear contribution to storage compliance at fundamental frequency during high field excitation $\left[\Delta \ln \left(\varepsilon^{\prime} / \sigma_{0}\right)_{\omega}\right]_{h f}$ determined by KWW-fits based on eq. 3.24 plotted vs. squared high field stress amplitude $\sigma_{0}^{2}$ for several temperatures. Lines correspond to linear fits, shaded areas to the uncertainty of the respective fit.

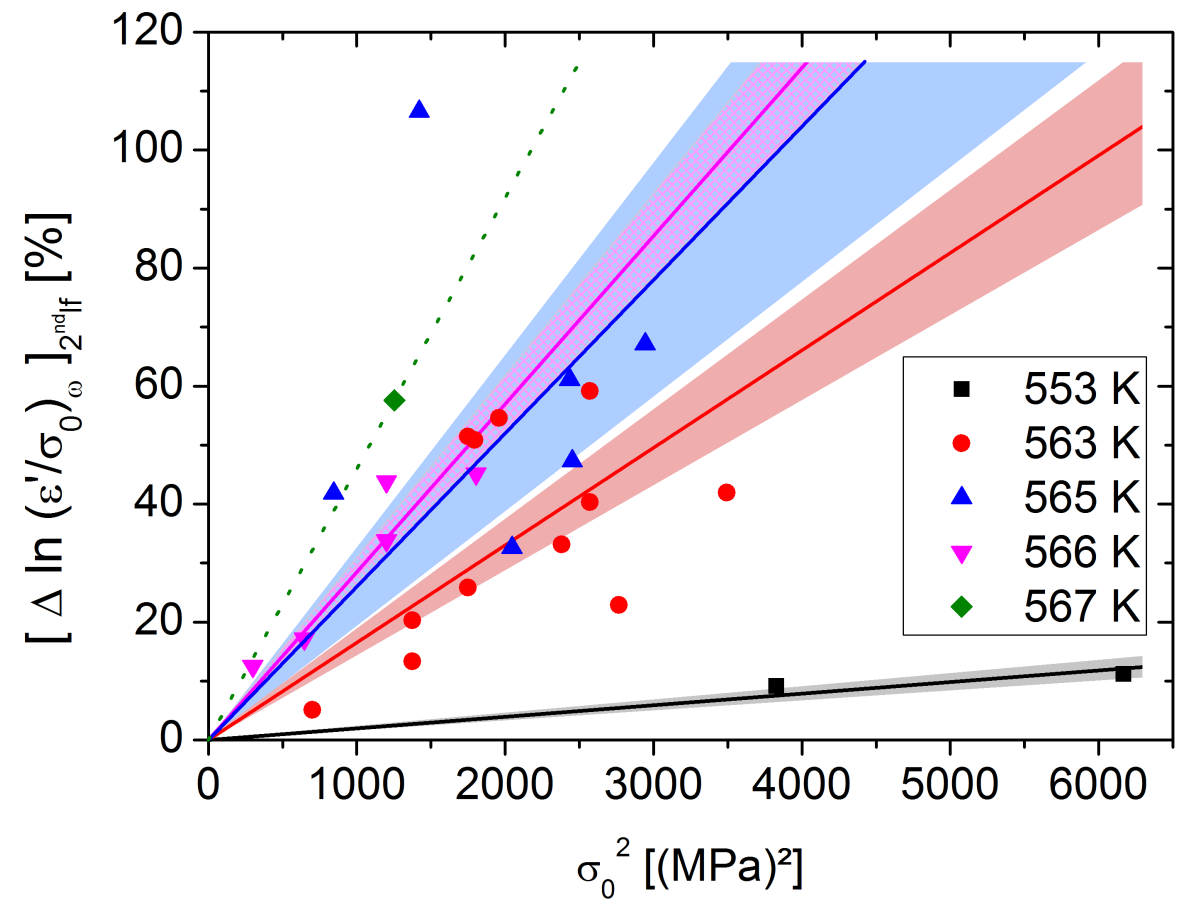

Figure 5.8 Steady state amplitude of relative nonlinear contribution to storage compliance at fundamental frequency during second low field excitation $\left[\Delta \ln \left(\varepsilon^{\prime} / \sigma_{0}\right)_{\omega}\right]_{2^{\text {nd }} \text { If }}$ determined by KWW fits based on eq. 3.25 plotted vs. squared high field stress amplitude $\sigma_{0}^{2}$ for several temperatures. Lines correspond to linear fits, shaded areas to the uncertainty of the respective fit. 


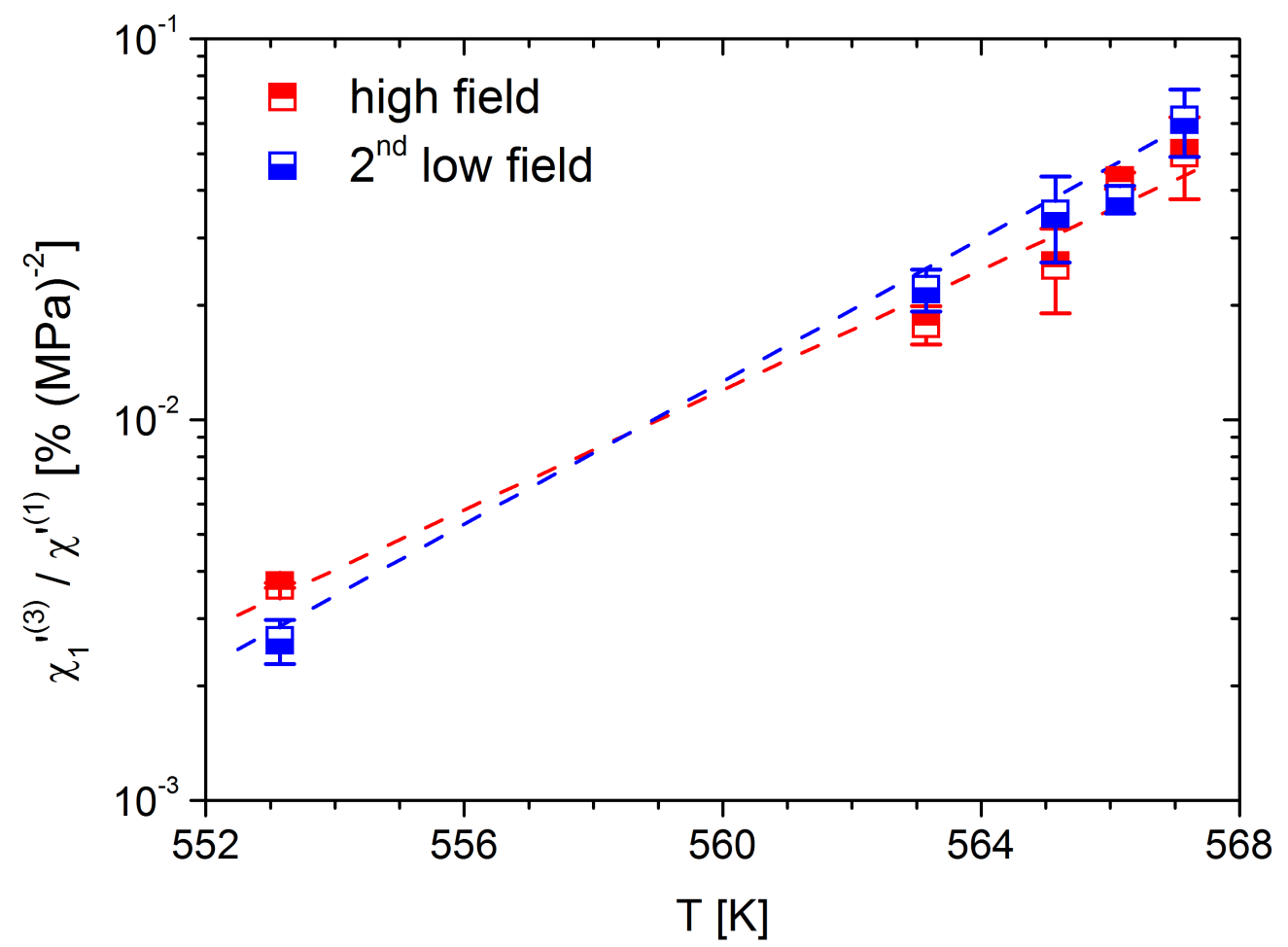

Figure 5.9 Relative nonlinear coefficients $\chi_{1}^{\prime(3)} / \chi^{\prime(1)}$ based on data shown in fig. 5.7 and fig. 5.8 plotted in logarithmic scaling against temperature $T$. High field and second low field data is fitted by linear regressions (dashed lines), see text for the resulting slopes and ordinates.

\section{Influence of Stress and Temperature on the Steady State Amplitudes of Relative Non- linear Storage Compliance}

Fig. 5.7 and fig. 5.8 show the extracted plateau-values of $\Delta \ln \left(\varepsilon^{\prime} / \sigma_{0}\right)_{\omega}$ during high field and second low field. According to eq. 3.21, this value for the relative nonlinear contribution at fundamental frequency shows a linear relation towards squared high field stress $\sigma_{0}^{2}$ with a slope equal to $\frac{3}{4} \chi_{1}^{(3)} / \chi^{(1)}$ and thus proportional to the nonlinear coefficient $\chi_{1}^{(3)}$. To determine the nonlinear coefficient $\chi_{1}^{\prime(3)} / \chi^{\prime(1)}$, the data is fitted using a linear regression through zero for each temperature.

The resulting relative nonlinear coefficients are plotted versus temperature in fig. 5.9 in semi-logarithmic scaling. A linear dependence of $\log \left(\chi_{1}^{(3)^{\prime}} / \chi^{(1)^{\prime}}\right)$ on temperature is clearly observed in the investigated temperature range. By means of linear regression the slope and ordinate values are extracted.

For high-field data the slope is determined to $0.079(8) \log \left(\%(\mathrm{MPa})^{-2}\right) \mathrm{K}^{-1}$ with an ordinate of $-46(4) \log \left(\%(\mathrm{MPa})^{-2}\right)$. For low-field data the slope is $0.094(4) \log \left(\%(\mathrm{MPa})^{-2}\right) \mathrm{K}^{-1}$ and the ordinate has a value of $-55(3) \log \left(\%(\mathrm{MPa})^{-2}\right)$. 


\section{Temperature-Dependent Timescales of Relative Nonlinear Storage Compliance}

To investigate the influence of temperature on the timescale extracted by the KWW-fits, fig. 5.10 shows mean values of timescales of high and low field data, $\tau_{h f}$ and $\tau_{2^{\text {nd }}} / f$, plotted against temperature. The average of $\tau_{h f}$ and $\tau_{2^{\text {nd }} \text { lf }}$ is depicted as $\tau_{\text {mean }}$. To allow for a comparison with literature data on the $\alpha$-relaxation timescale of $\mathrm{Pd}_{40} \mathrm{Ni}_{40} \mathrm{P}_{20}$, green symbols show values of the temperature-dependent $\tau_{\alpha}$ deduced from viscosity data [151] via eq. 2.15. The viscosity data are measured by creep-experiments after sufficient annealing of the samples to reach a metastable equilibrium. The average timescales $\tau_{h f}, \tau_{2^{\text {nd }} / f}$, and $\tau_{\text {mean }}$ show a rather constant behavior. The timescales deduced at a temperature of $553 \mathrm{~K}$, depicted at $T / T_{g}=1.08$, is close to the timescale of structural relaxation. For higher temperatures the timescales extracted from the LAOS-measurements show values which are one order of magnitude higher than the structural relaxation timescale.

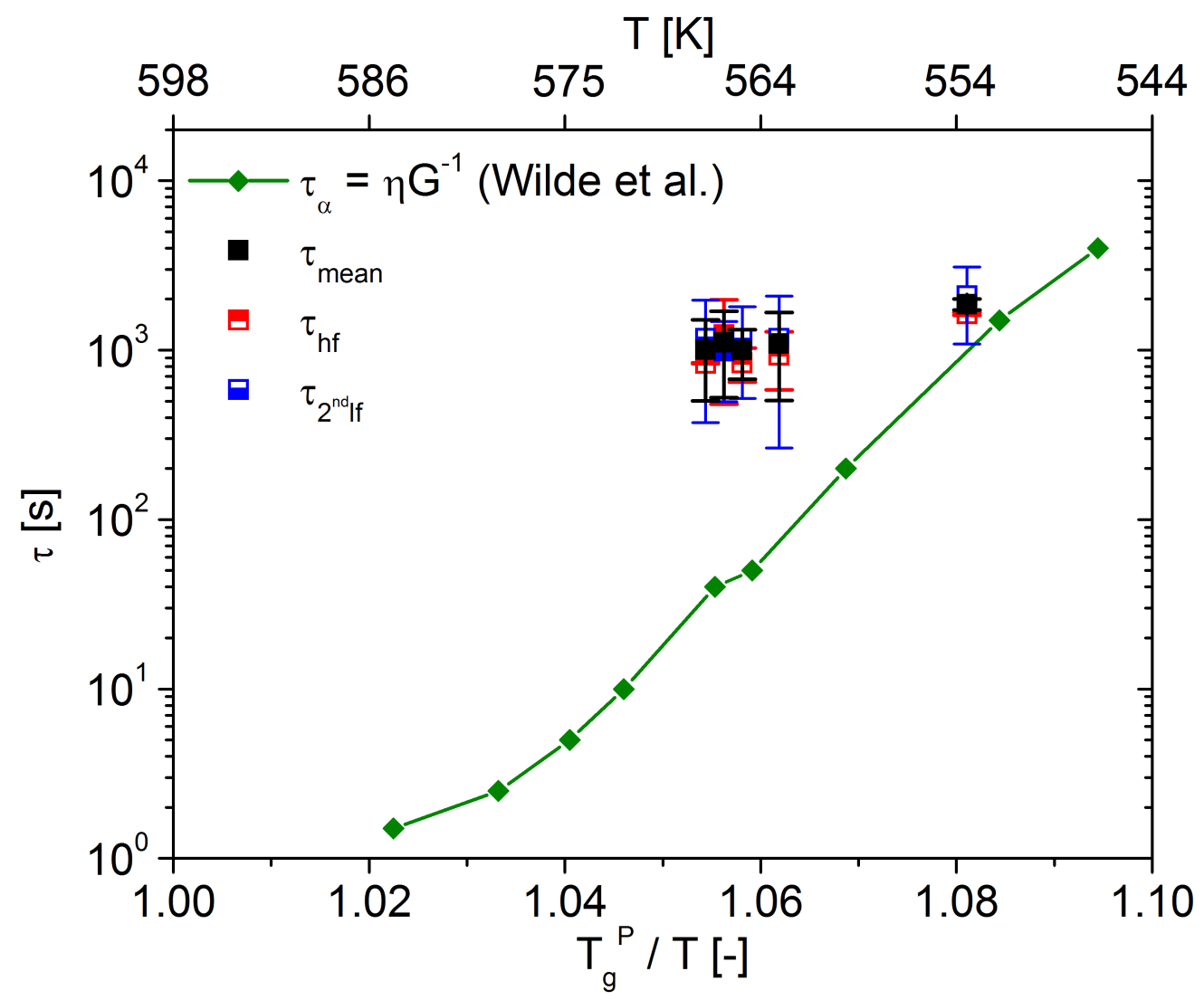

Figure 5.10 Average timescales, $\tau_{\text {mean }}, \tau_{h f}$, and $\tau_{2^{\text {nd }} / f}$, based on high field and second low field relative nonlinear storage compliance $\Delta \ln \left(\varepsilon^{\prime} / \sigma_{0}\right)_{\omega}$ plotted versus temperature $T$. Green symbols with line correspond to literature data by [151]. 


\subsubsection{Nonlinear Contribution of Loss Compliance at Fundamental Frequency during and after High Field Excitation}

As described in section 5.3.1 the nonlinear contribution to compliance under high field excitation at the fundamental frequency $\Delta \ln \left(\varepsilon / \sigma_{0}\right)_{\omega}$ gives insights into the response behavior of metallic glasses under high stress at elevated temperature. In this chapter the loss part of this quantity, $\Delta \ln \left(\varepsilon^{\prime \prime} / \sigma_{0}\right)_{\omega}$, shall be investigated. It is determined by eq. 3.13 and data typical for measurements at temperatures between $553 \mathrm{~K}$ and $567 \mathrm{~K}$ are plotted in fig. $5.11 \mathrm{a}$. After the initial low field response between $-3600 \mathrm{~s}$ and $0 \mathrm{~s}$, which shows constant steady state behavior, a sudden rise in compliance occurs with the onset of high field excitation. The compliance rises further and finally saturates at a new steady state value. Even though absolute values in loss and storage compliance differ a lot as loss compliance $\left(\varepsilon^{\prime \prime} / \sigma_{0}\right)_{\omega}$ is around $10 \%$ of $\left(\varepsilon^{\prime} / \sigma_{0}\right)_{\omega}$, the relative nonlinear loss compliance $\Delta \ln \left(\varepsilon^{\prime \prime} / \sigma_{0}\right)_{\omega}$ is of comparable order of magnitude to $\Delta \ln \left(\varepsilon^{\prime} / \sigma_{0}\right)_{\omega}$. Still, the low absolute values in loss compliance lead to rather noisy data for $\Delta \ln \left(\varepsilon^{\prime \prime} / \sigma_{0}\right)_{\omega}$ after high field excitation (3601 s to $7200 \mathrm{~s}$ ). It is observed that the relative nonlinear loss compliance $\Delta \ln \left(\varepsilon^{\prime \prime} / \sigma_{0}\right)_{\omega}$ jumps to an instantaneous value with the beginning of the second low field excitation and decreases from this initial point to a low-field steady state value.

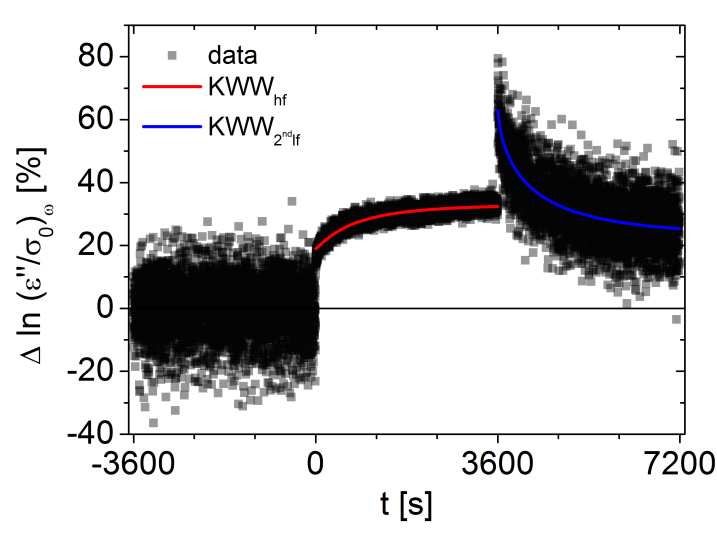

(a) $563 \mathrm{~K}$ to $567 \mathrm{~K}$

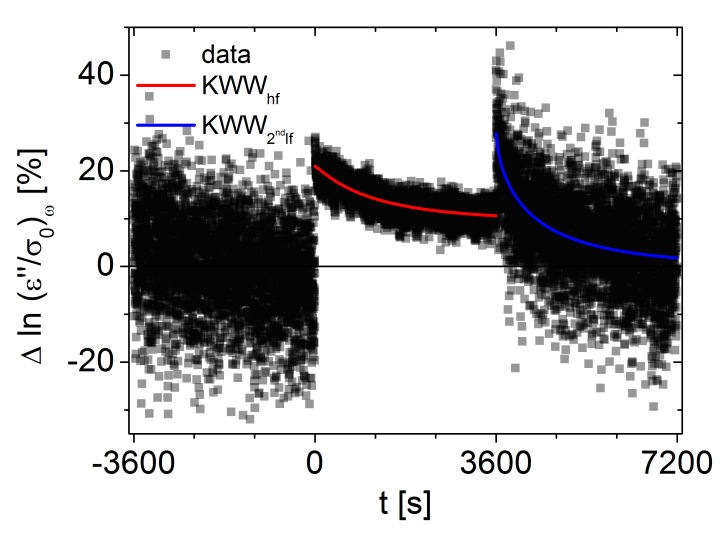

(b) $553 \mathrm{~K}$

Figure 5.11 Examples for time-dependent behavior of nonlinear relative change in loss compliance at fundamental frequency $\Delta \ln \left(\varepsilon^{\prime \prime} / \sigma_{0}\right)_{\omega}$. (a) corresponds to data measured at $T=563 \mathrm{~K}$ and $\sigma_{0}=51 \mathrm{MPa}$, typical for measurements in the temperature range of $563 \mathrm{~K}$ and $567 \mathrm{~K}$. (b) is based on a measurement at $553 \mathrm{~K}$ and $\sigma_{0}=79 \mathrm{MPa}$.

The relative nonlinear loss compliance $\Delta \ln \left(\varepsilon^{\prime \prime} / \sigma_{0}\right)_{\omega}$ at a temperature of $553 \mathrm{~K}$ shows reproducible behavior of a different kind: Under initial low field excitation the signal mildly decays where measurements at higher temperatures show a plateau. During high-field excitation the loss signal shows a decrease instead of an increase towards a new steady state (cf. fog. 5.11b). This behavior is not observed for the storage part. During second low field excitation the relative loss response shows an instantaneous jump followed by a decay as observed in measurements at higher temperatures as well. Comparable to the data analysis for the storage part the data are fitted using squared $\mathrm{KWW}$-functions as described in equations 3.24 and 3.25 . 


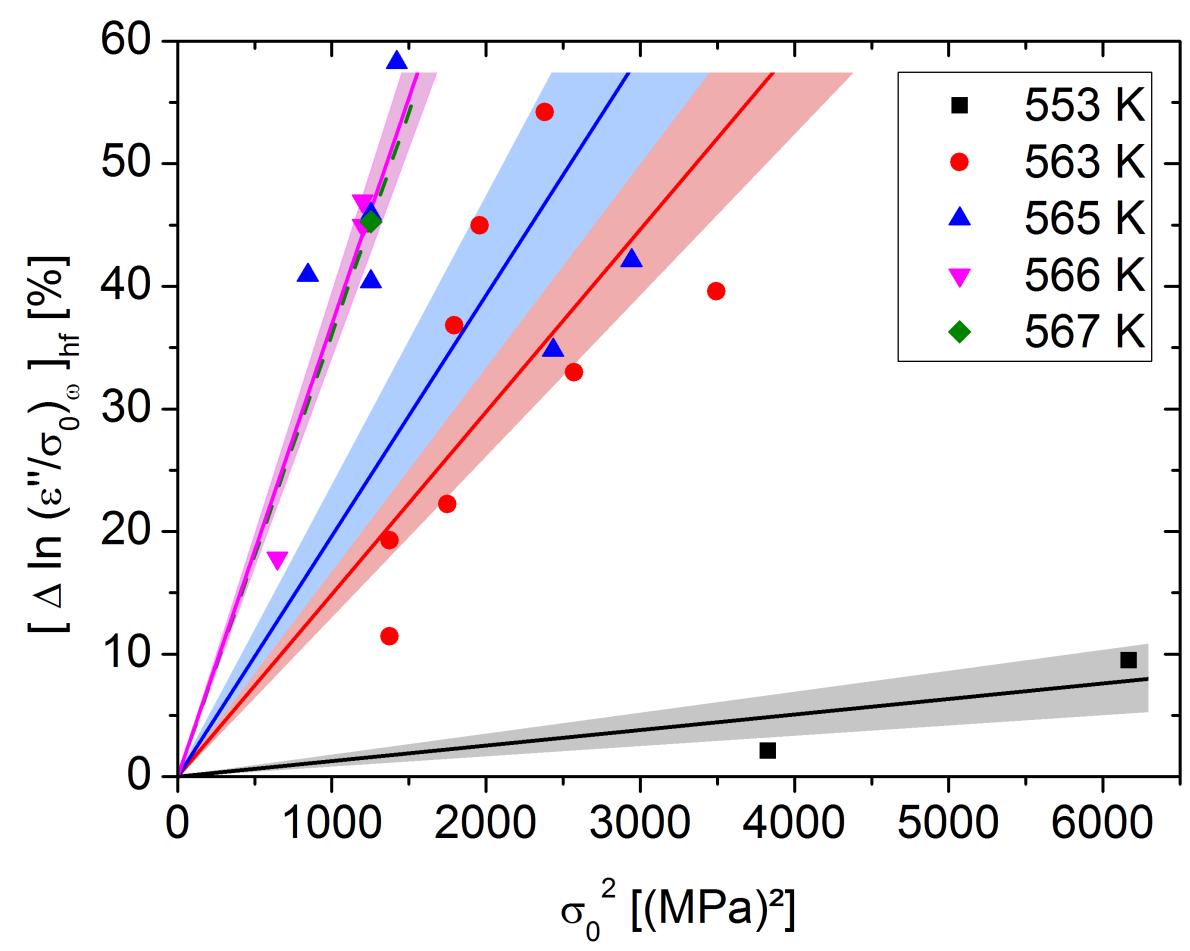

Figure 5.12 Steady state values of relative nonlinear contribution to loss compliance at fundamental frequency during high field excitation $\left[\Delta \ln \left(\varepsilon^{\prime \prime} / \sigma_{0}\right)_{\omega}\right]_{h f}$ determined by KWW-fits based on eq. 3.24 plotted vs. squared high field stress amplitude $\sigma_{0}^{2}$ for several temperatures. Lines correspond to linear fits, shaded areas to the uncertainty of the respective fit.

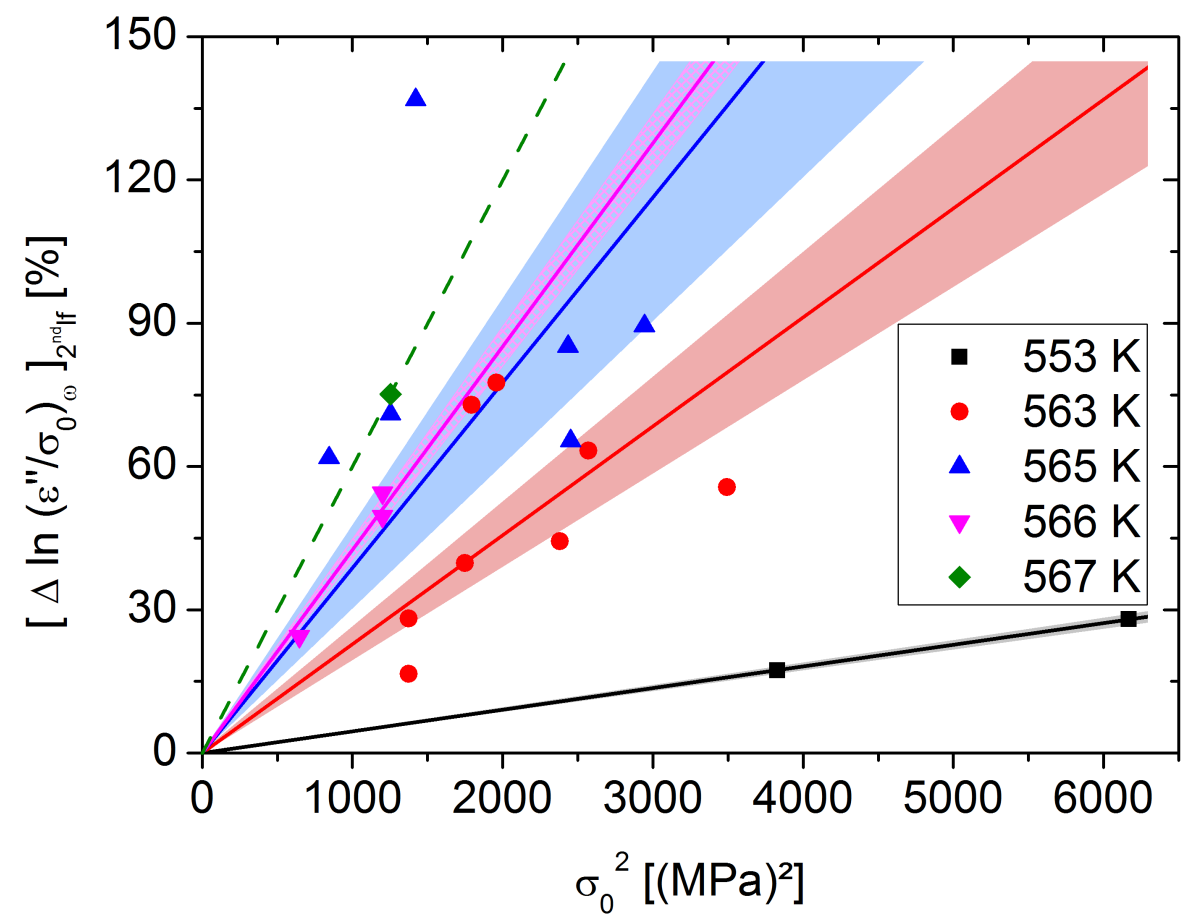

Figure 5.13 Steady state values of relative nonlinear contribution to loss compliance at fundamental frequency during second low field excitation $\left[\Delta \ln \left(\varepsilon^{\prime \prime} / \sigma_{0}\right)_{\omega}\right]_{2^{\text {nd }} / f}$ determined by KWW-fits based on eq. 3.25 plotted against squared high field stress amplitude $\sigma_{0}^{2}$ for different temperatures. Lines correspond to linear fits, shaded areas to the uncertainty of the respective fit. 
Influence of Stress and Temperature on the Steady State Amplitudes of Relative Nonlinear Loss Compliance

As in the case of relative nonlinear storage compliance, a linear relation to the squared high field stress $\sigma_{0}^{2}$ is expected for the steady state value of the loss $\left[\Delta \ln \left(\varepsilon^{\prime \prime} / \sigma_{0}\right)_{\omega}\right]$ (eq. 3.21). To evaluate this relation, steady state values of $\Delta \ln \left(\varepsilon^{\prime \prime} / \sigma_{0}\right)_{\omega}$ at high field and second low field are extracted by KWW-fits. These steady state values are shown in fig. 5.12 for high field data and in fig. 5.13 for second low field data. In both figures, the expected linearity can be seen more or less pronounced depending on the temperature evaluated. The slopes extracted in fig. 5.12 and 5.13 are proportional to the relative nonlinear coefficient $\chi_{1}^{\prime \prime(3)} / \chi^{\prime \prime(1)}$. It yields a linear behavior in a semi-logarithmic plot versus temperature (fig. 5.14). For evaluation of high-field data by a linear regression the slope can be determined to $0.106(6) \log \left(\%(\mathrm{MPa})^{-2}\right) \mathrm{K}^{-1}$ with an ordinate of $-61(4) \log \left(\%(\mathrm{MPa})^{-2}\right)$. For evaluation of second low-field data the slope is $0.078(4) \log \left(\%(\mathrm{MPa})^{-2}\right) \mathrm{K}^{-1}$ and the ordinate has a value of $-45(3) \log \left(\%(\mathrm{MPa})^{-2}\right)$.

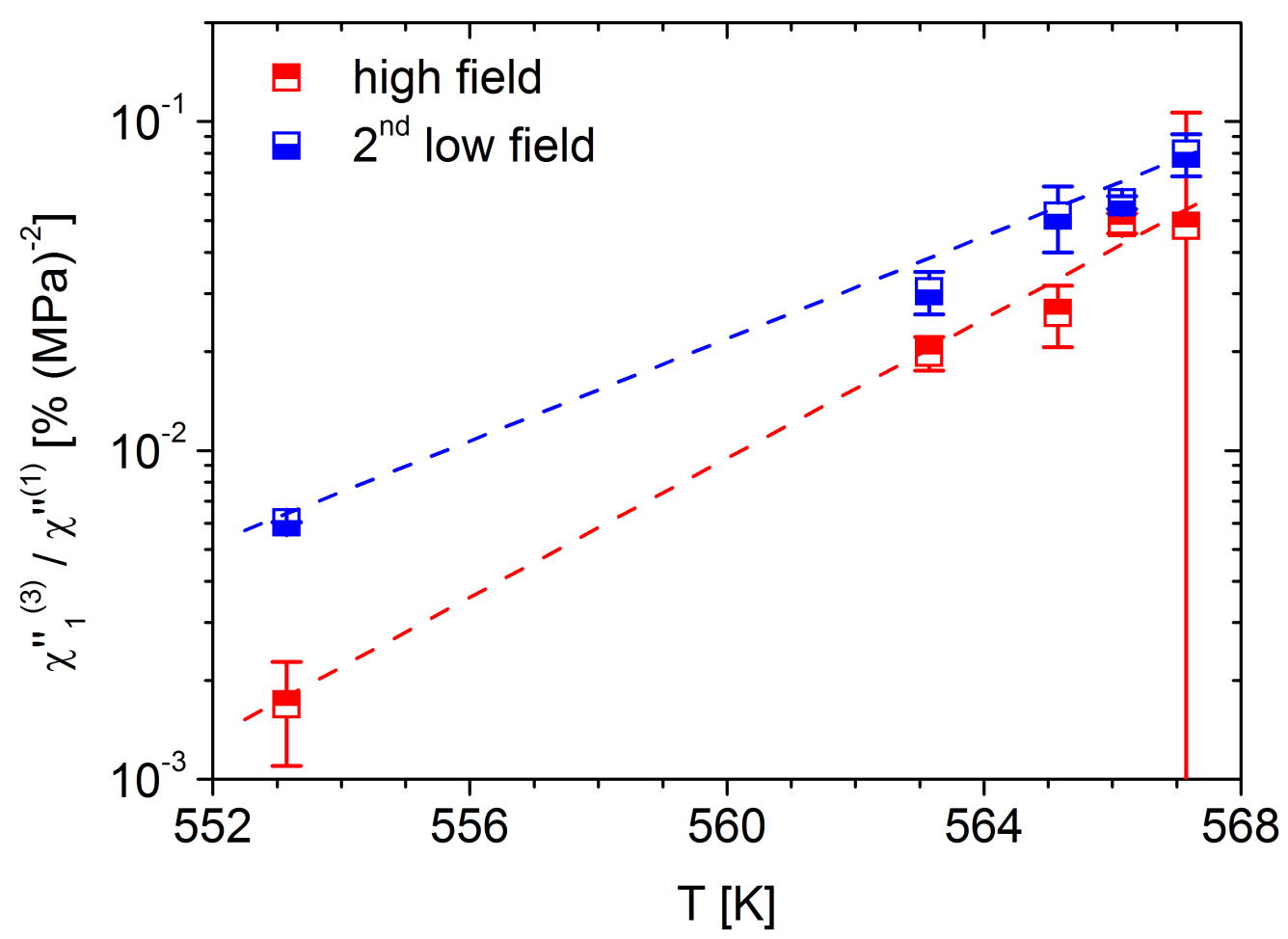

Figure 5.14 Relative nonlinear coefficients $\chi_{1}^{\prime \prime(3)} / \chi^{\prime \prime(1)}$ extracted from data shown in fig. 5.12 and fig. 5.13 plotted in logarithmic scaling against temperature $T$. High field and second low field data is fitted by linear regressions (lines), see text for slopes and ordinates. 


\section{Temperature-Dependent Timescales of Relative Nonlinear Loss Compliance}

Timescales extracted by KWW-fits at high field $\left(\tau_{h f}\right)$ and second low field $\left(\tau_{2^{\text {nd }} / f}\right)$ are averaged over all measurements of the same temperature and plotted in fig. 5.15. The mean of both high field and second low field timescales is denoted as $\tau_{\text {mean. }}$. Again, to allow for a comparison with literature data on the $\alpha$-relaxation timescale, green symbols show values of the temperature-dependent $\tau_{\alpha}$ deduced from viscosity data [151] via eq. 2.15, comparable to the data shown in fig. 5.10

At temperatures between $563 \mathrm{~K}$ and $567 \mathrm{~K}$, i.e. between $T / T_{g}$ between 1.05 and $1.07, \tau_{h f}$ and $\tau_{2^{\text {nd }}}$ /f are mildly temperature-dependent and decreasing with increasing temperature. Timescales measured under high field $\tau_{h f}$ are about one order of magnitude below corresponding second low field data. Thus, the mean timescale $\tau_{\text {mean }}$ is between one and two orders of magnitude above the literature data, which represents Vogel-Flucher-Tammann behavior.

$\tau_{h f}$ and $\tau_{2^{\text {nd }} / f}$ at $553 \mathrm{~K}$, i.e. $T / T_{g}=1.08$ are very close and of the same order of magnitude as mean timescales measured for higher temperatures. The timescales extracted from the experiments are of the same order as the literature data for $\tau_{\alpha}$ measured by Wilde et al. [151].

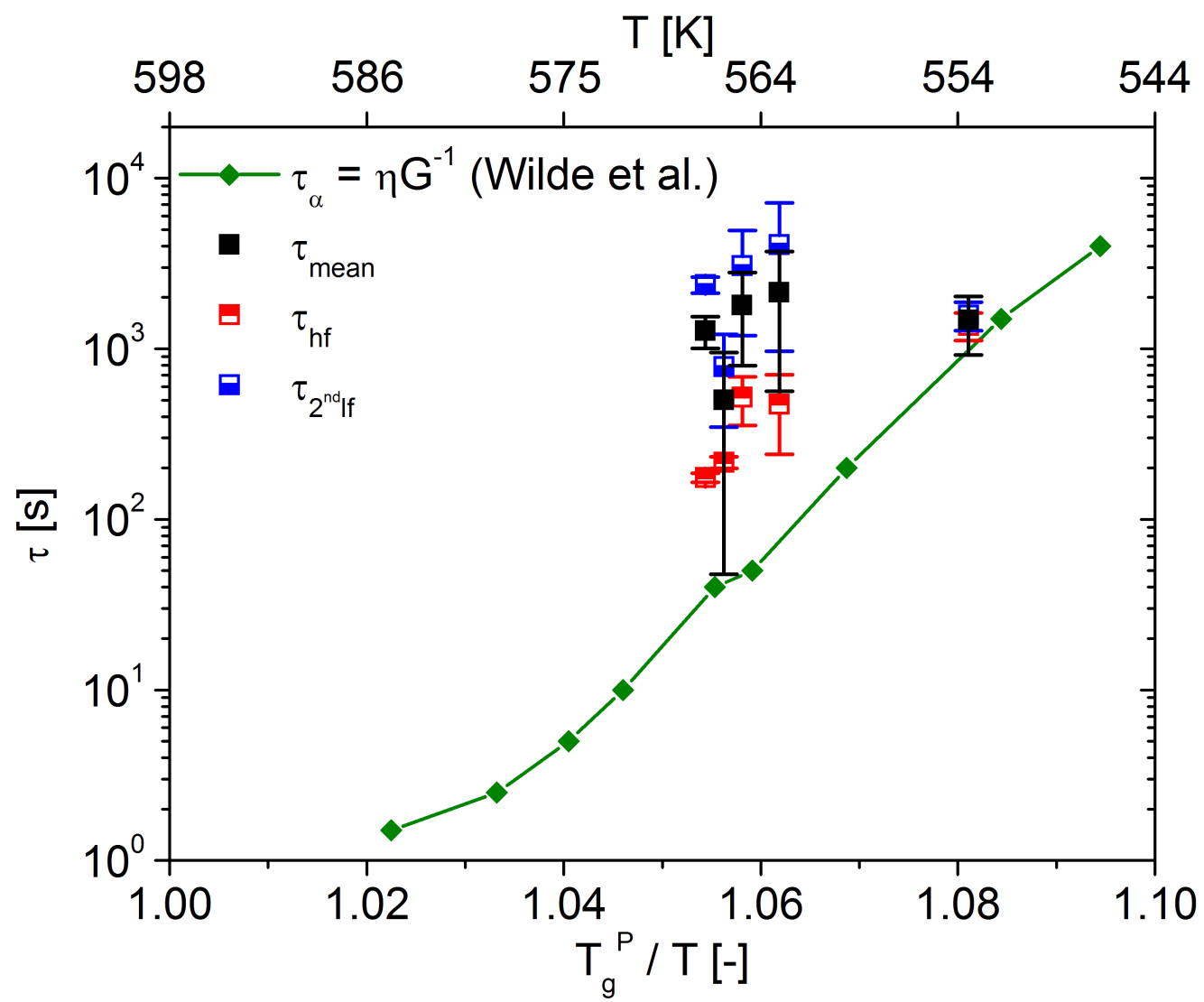

Figure 5.15 Average timescales, $\tau_{h f}, \tau_{2^{\text {nd }} / f}$, and $\tau_{\text {mean }}$, based on high field and second low field relative nonlinear loss compliance $\Delta \ln \left(\varepsilon^{\prime \prime} / \sigma_{0}\right)_{\omega}$ plotted versus temperature $T$. Green symbols with line correspond to literature data by [151]. 


\subsubsection{Nonlinear Contributions of Compliance at Higher Harmonic Frequency}

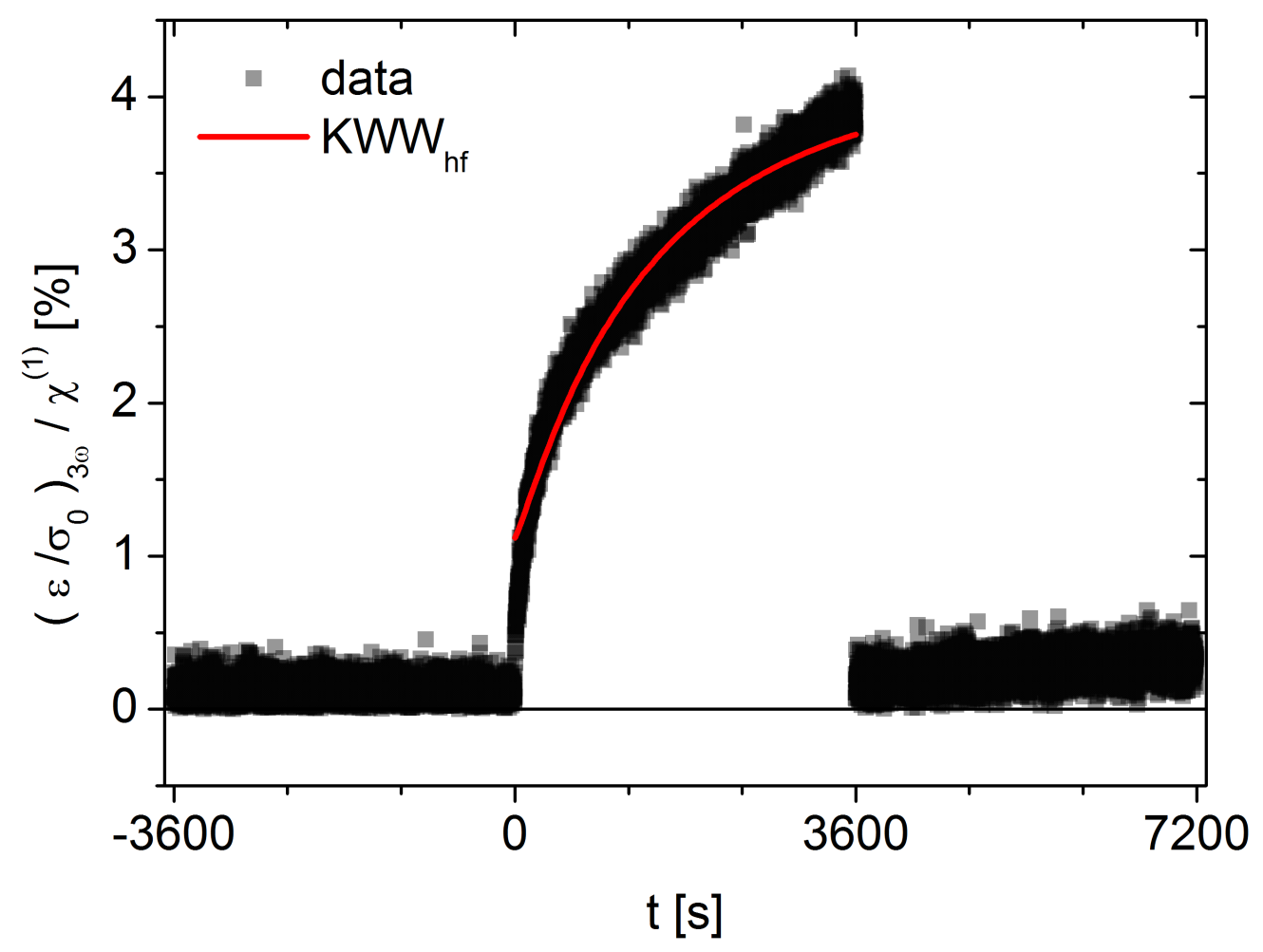

Figure 5.16 Example for time-dependent behavior of $\frac{\left(\epsilon / \sigma_{0}\right)_{3 \omega}}{\chi^{(1)}}$ at $T=563 \mathrm{~K}$ and $\sigma_{0}=10 \mathrm{MPa}$.

A typical feature of nonlinear response behavior due to sinusoidal excitation at high excitation amplitude are odd higher-harmonic contributions. To evaluate the impact of mechanical stress on their appearance, the relative third harmonic compliance $\left(\epsilon / \sigma_{0}\right)_{3 \omega} / \chi^{(1)}$ is determined according to eq. 3.23 . The compliance $\chi$ is equal to the absolute value of the complex compliance $\hat{\chi}$. If the relative third harmonic compliance quantity is plotted versus time as in fig. 5.16, parameters as the timescale and the steady state amplitude can be extracted by fitting with a squared KWW-function.

\section{Influence of Stress and Temperature on Steady State Amplitudes of Relative Nonlinear Third Harmonic Compliance}

As for the compliance at fundamental frequency, the sum of instantaneous and continuous amplitude yields the steady state value for high field. The quantity $\left(\epsilon / \sigma_{0}\right)_{3 \omega} / \chi^{(1)}$ is linearly dependent on the square of applied high field stress (cf. eq. 3.23). Thus linear regressions are calculated by the least square method with a fixed ordinate of zero for individual temperatures as shown in fig. 5.17. The linear dependence of the steady state amplitudes reveals an increase in the relative nonlinear coefficient with temperature and an exponential behavior of $\log \left(\chi_{3}^{(3)} / \chi^{(1)}\right)$ versus temperature $T$ is observed. An exponential growth function $y=y_{0}+$ $y_{1} \cdot \exp \left(\frac{\left(x-x_{0}\right)}{\gamma}\right)$ was used to fit the data. Therefore, the amplitude was set to $y_{1}=8.910^{-18}$, free fit parameters are the offset $y_{0}=0.0079(2)$, the shift parameter of $x_{0}=546.4$ (9) and the exponent of $\gamma=0.58(2)$. In comparison to relative nonlinear coefficients at fundamental frequency, the exponent $\gamma$ deviates clearly from a linear relation between logarithmic relative coefficient and temperature. Technically, this is due to the offset $y_{0}$, which does not fulfill the approximation $y_{0}<<y$ in the case of the fit for third harmonic frequency data. 


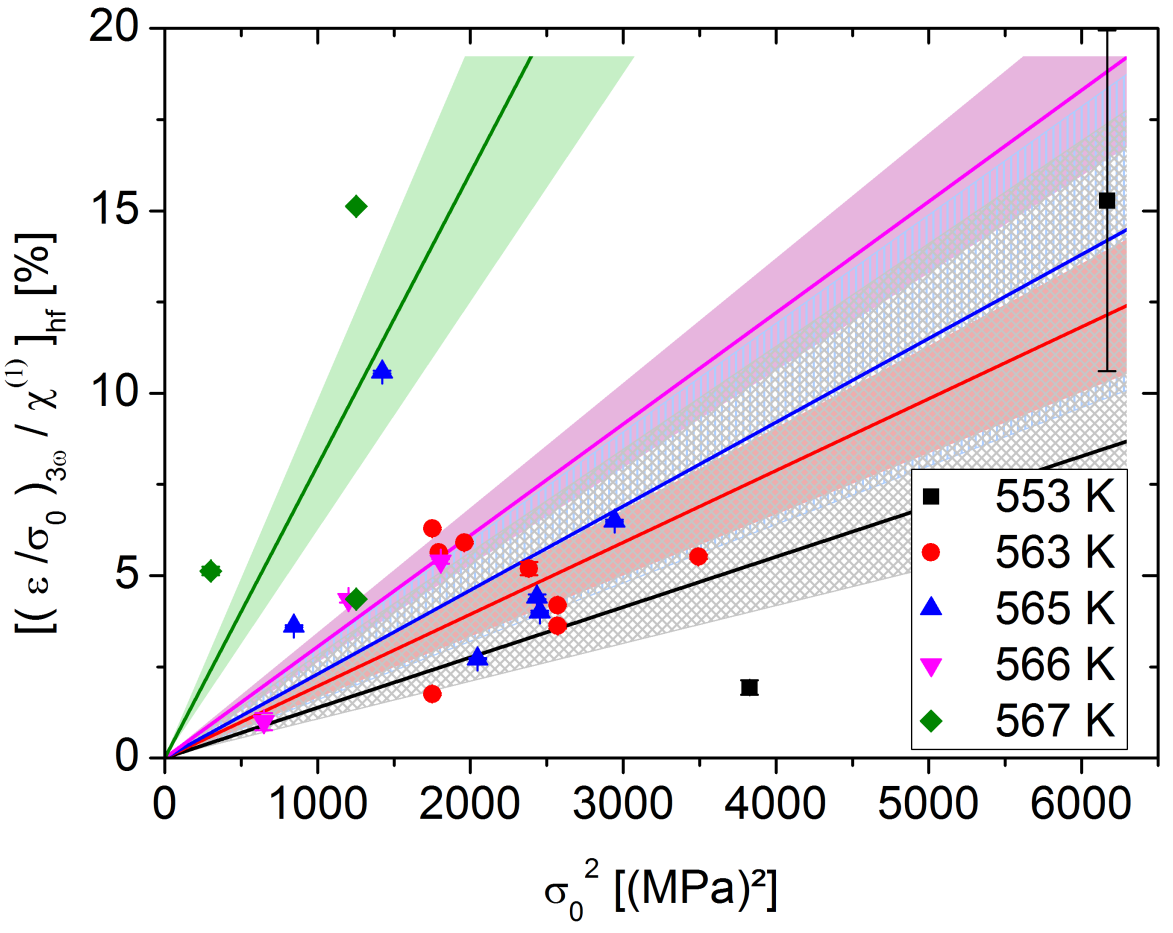

Figure 5.17 Steady state values of relative complex compliance at third harmonic frequency during high field excitation $\left[\left(\epsilon / \sigma_{0}\right)_{3 \omega} / \chi^{(1)}\right]_{h f}$ determined by KWW-fits plotted against squared high field stress amplitude $\sigma_{0}^{2}$ for several temperatures. Lines correspond to linear fits, shaded areas to the uncertainty of the respective fit.

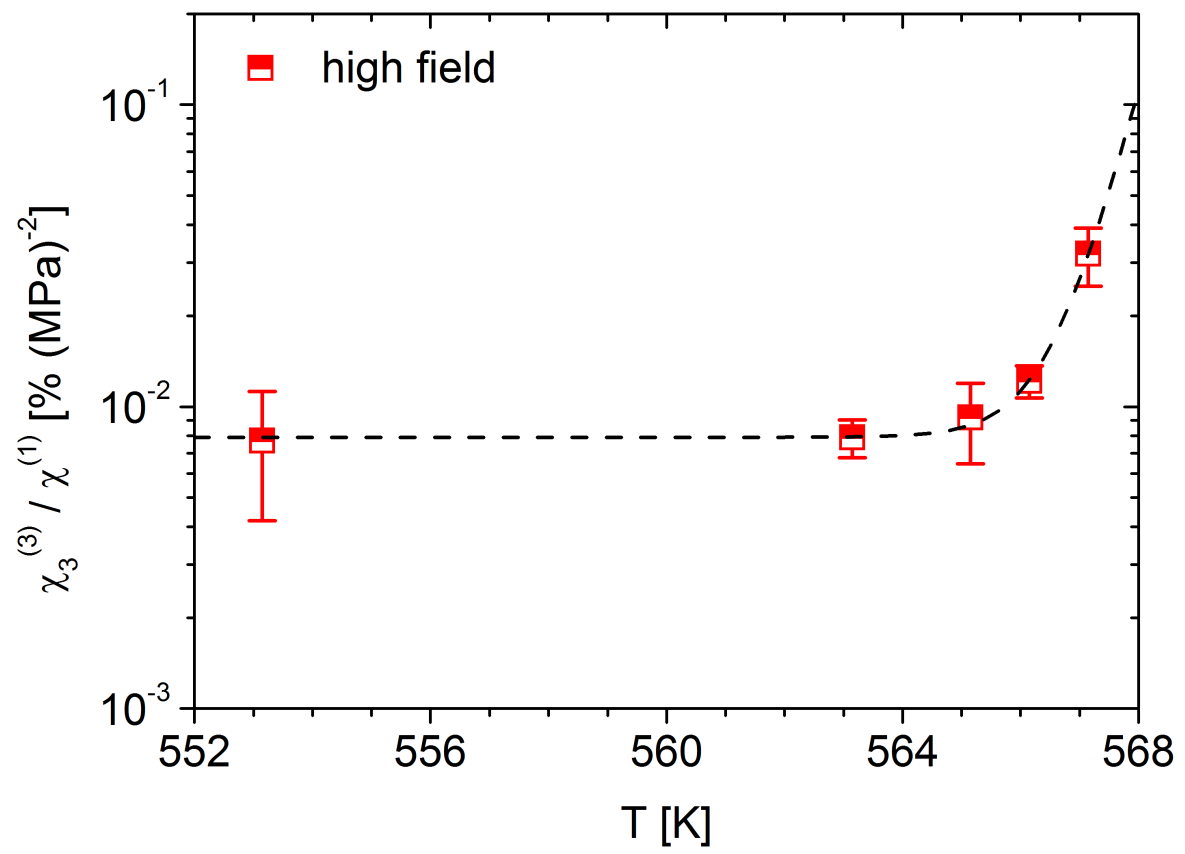

Figure 5.18 Relative nonlinear coefficient $\chi_{3}^{(3)} / \chi^{(1)}$ based on data shown in fig. 5.17 plotted against temperature $T$. The black dashed line resembles an exponential fit, see text for details on the fit function and for fit parameters. 
Temperature-Dependent Timescales of Relative Nonlinear Third Harmonic Compliance

The time-dependence of the response signal at the third harmonic frequency is represented by the timescale $\tau_{h f}$. It is extracted by KWW-fits and is plotted against inverse temperature in fig. 5.19. The timescale shows a temperature-dependence similar to the VFT behavior of the literature data (green symbols with line), but are in general one order of magnitude larger than the data on the structural relaxation timescale. However, the standard deviations of the averages are high compared to the difference between average values at different temperatures.

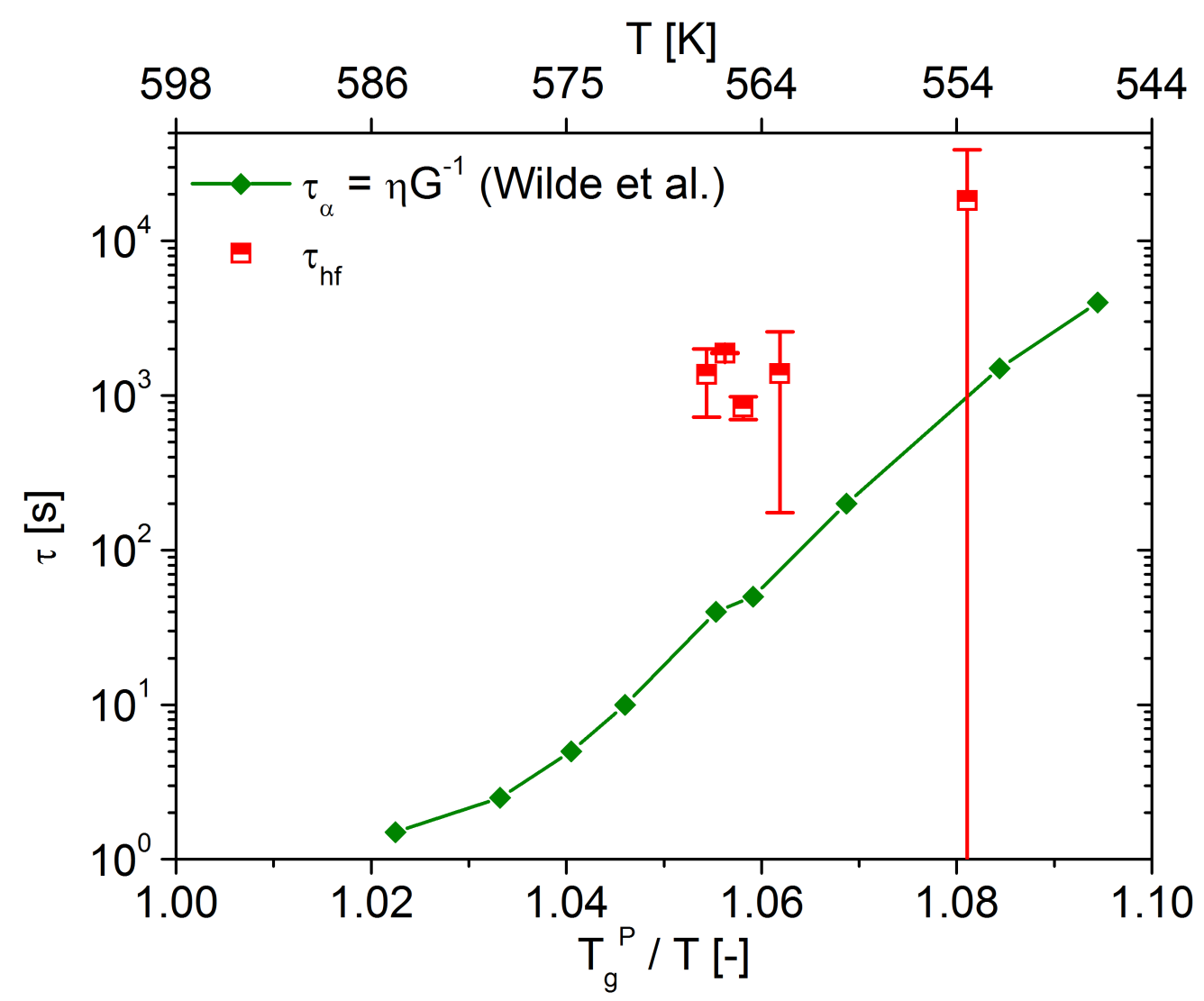

Figure 5.19 Average timescales $\tau_{h f}$ of $\chi_{3 \omega} / \chi^{(1)}$ plotted versus temperature $T$. The green symbols with line correspond to literature data by [151]. 


\subsection{Incremental Analysis}

As studied numerically by Procaccia et al. [32], and observed in various other simulations [88, 89] and experiments on colloidal systems [122, 20, 19], the modulus is defined for a linear response behavior which is interrupted by breakdowns of elasticity as described by the EIP-motif [77]. Thus, from the view-point of avalanche dynamics and microscopic rearrangements, modulus and compliance are not expected to exhibit intrinsic higher order terms as described by a nonlinear expansion of the response behavior [110, 25]. Thus, the strain response obtained by LAOS-experiments is evaluated in regard to avalanche dynamics by means of an incremental analysis.

In the following chapter, the data accumulated in the LAOS-experiments is studied on the level of intervals, which are defined by the strain as explained in section 3.7.2. An interval is defined as the range of successive data points in strain which do either increase monotonously (positive intervals) or decrease monotonously (negative intervals). The strain values connected to such an interval are denoted as $\Delta \varepsilon_{i t v l}$. Moreover, the duration of strain intervals $\Delta t_{i t v l}$ in terms of time are analyzed. These interval-based quantities are discussed regarding their average values. These are extracted as described in section 3.7.2, and the dependence on temperature $T$, stress amplitude $\sigma_{0}$, and intra-periodic stress $\sigma_{c}$ is discussed.

In addition, the distributions of strain intervals in dependence on the afore mentioned parameters are investigated in regard to power law behavior. 


\subsubsection{Strain Rate}

As described in section 3.7 .1 the average strain rate $\dot{\varepsilon}$ is extracted as a mean value over 50 data points and plotted for a temperature of $563 \mathrm{~K}$ in fig. $5.20 \mathrm{a}$. The strain rate shows a decrease on the intra-periodic scale. With increasing stress amplitude $\sigma_{0}$ the strain rate increases, as is observed very clearly at low intra-periodic stresses. By normalization with the maximum value of strain rate, all measurements collapse onto a mastercurve (fig. 5.20a). The maximum strain rate which serves as the normalization factor is plotted in fig. 5.21 against the stress amplitude $\sigma_{0}$ for various temperatures. For the lowest temperature of $553 \mathrm{~K}$ a linear dependence of $\dot{\varepsilon}$ on $\sigma_{0}$ is observed. Linear regression yields a slope of $9.5(1) \times 10^{-5} \mathrm{MPa}^{-1} \mathrm{~s}^{-1}$ and an ordinate of $-1.5(4) \times 10^{-4} \mathrm{~s}^{-1}$. For higher temperatures a linear dependence of the strain rate is observed for low stress amplitudes, but a deviation towards higher strain rates is evident with increasing stress amplitude $\sigma_{0}$.

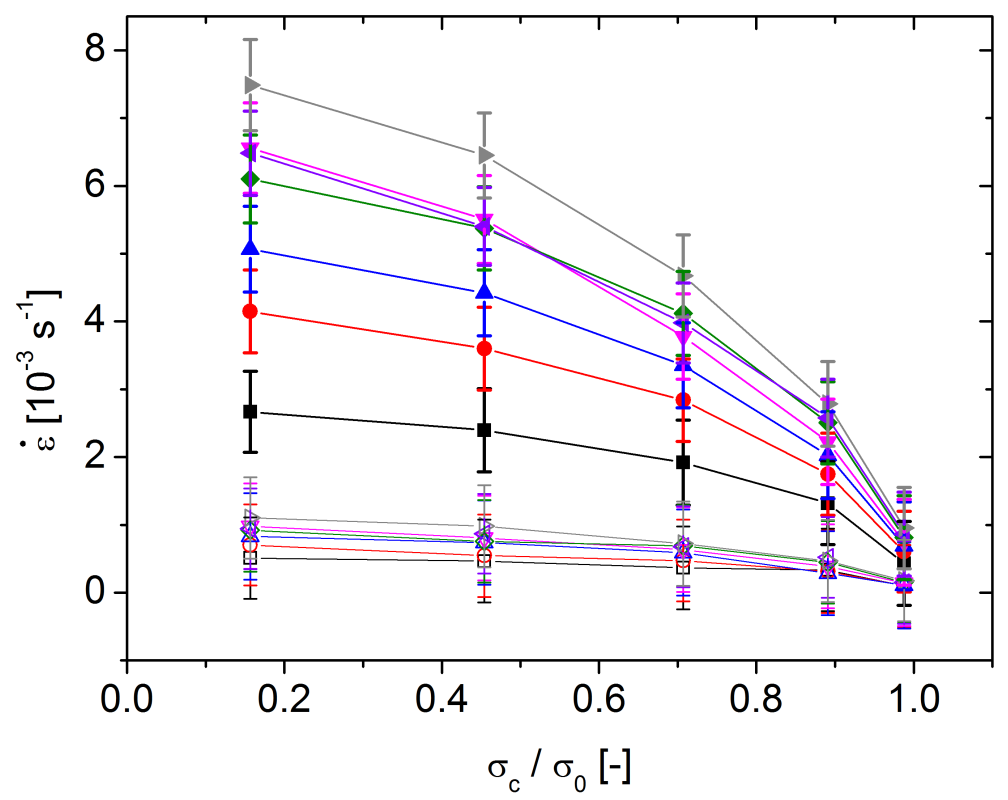

(a)

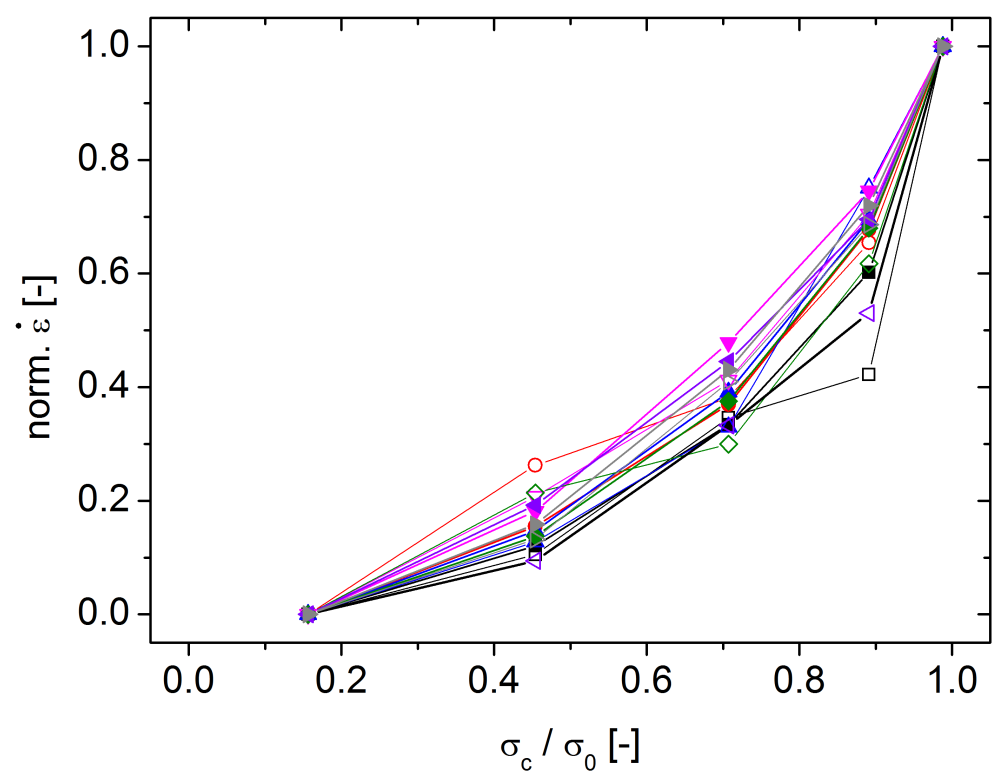

\begin{tabular}{|cc|}
\hline$\square$ & $5.29 \mathrm{MPa}$ \\
$\circ$ & $7.42 \mathrm{MPa}$ \\
$\triangle$ & $8.37 \mathrm{MPa}$ \\
$\nabla$ & $8.85 \mathrm{MPa}$ \\
$\diamond$ & $9.76 \mathrm{MPa}$ \\
$\triangleleft$ & $10.14 \mathrm{MPa}$ \\
$\triangleright$ & $11.82 \mathrm{MPa}$ \\
- & $26.47 \mathrm{MPa}$ \\
$\bullet$ & $37.10 \mathrm{MPa}$ \\
$\triangle$ & $41.85 \mathrm{MPa}$ \\
$\nabla$ & $44.27 \mathrm{MPa}$ \\
$\bullet$ & $48.82 \mathrm{MPa}$ \\
$\triangleleft$ & $50.71 \mathrm{MPa}$ \\
$\triangleright$ & $59.09 \mathrm{MPa}$ \\
\hline
\end{tabular}

(b)

Figure 5.20 Strain rate $\dot{\varepsilon}$ (a) and normalized strain rate $\dot{\varepsilon}$ (b) for various stress amplitudes $\sigma_{0}$ at constant temperature $T=563 \mathrm{~K}$. 


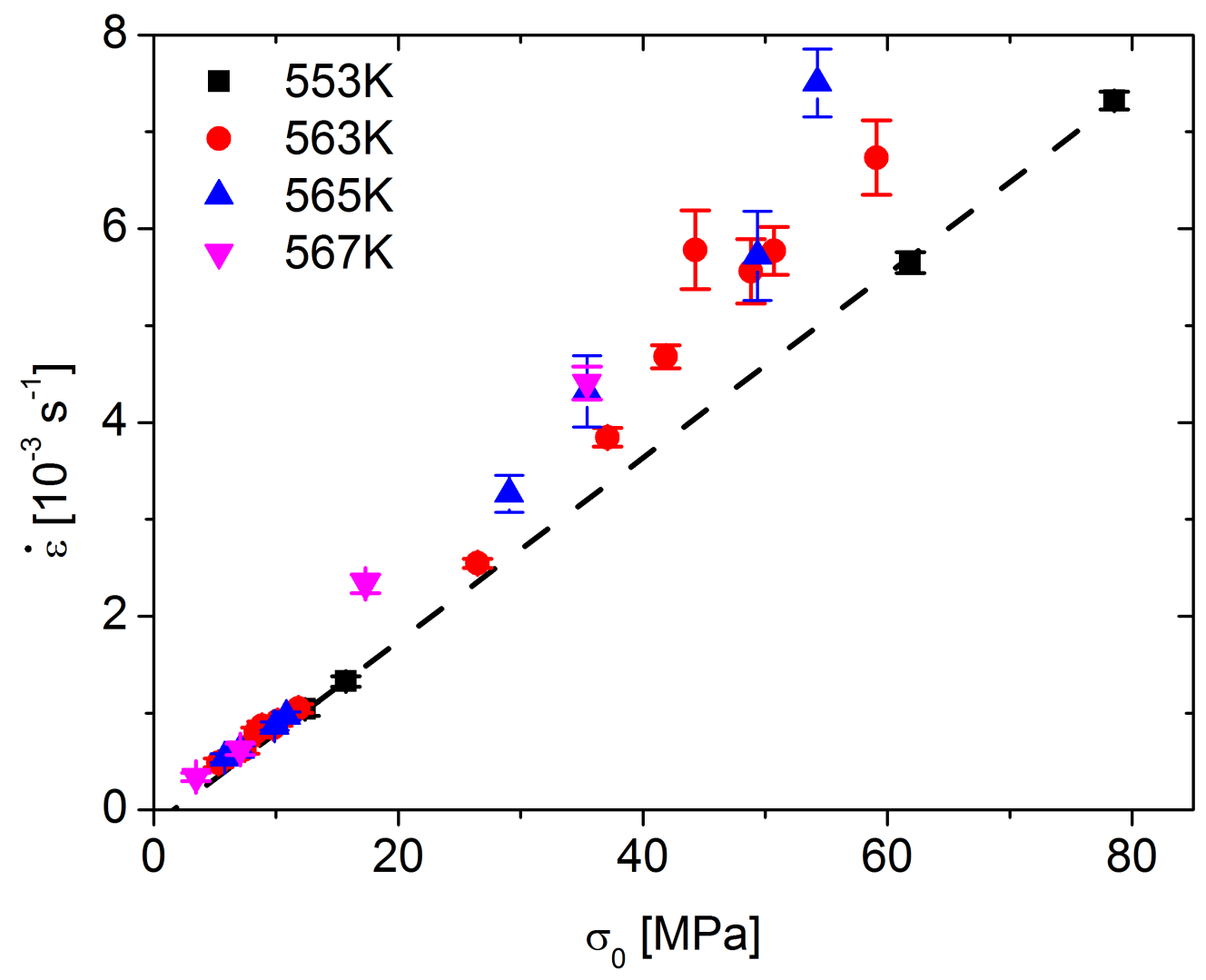

(a)

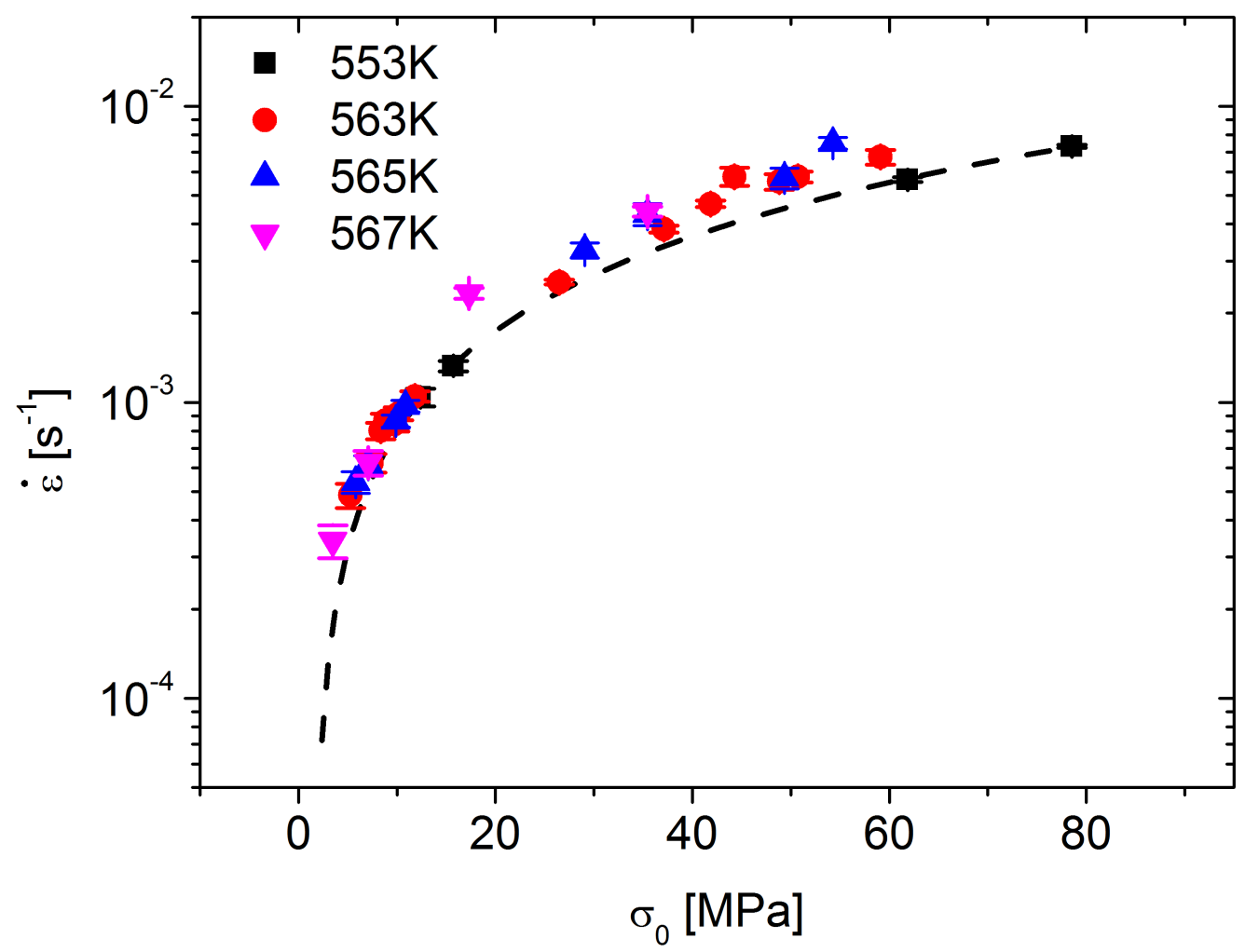

(b)

Figure 5.21 Linearly plotted strain rate $\dot{\varepsilon}$ (a) and logarithmically plotted strain rate $\dot{\varepsilon}$ (b) against stress amplitude $\sigma_{0}$ for various temperatures. The black dashed line represents a fit based on data plotted as in (a) measured at $553 \mathrm{~K}$ extracted by linear regression. 


\subsubsection{Averaged Values for Strain Intervals}

The averages of interval strain and interval duration are determined for strain intervals over stress windows of $2 \mathrm{MPa}$ width according to eq. 3.28 . The result is plotted against the mean of the stress window, that is denoted as the intra-periodic stress $\sigma_{c}$.

\section{Averages of Interval Strain}

The averaged interval strain for positive intervals is shown in fig. 5.22a for several stress amplitudes $\sigma_{0}$ measured at $563 \mathrm{~K}$. Open symbols represent strain data extracted from initial low field response, while solid symbols show averages of interval strain based on high field steady state data.

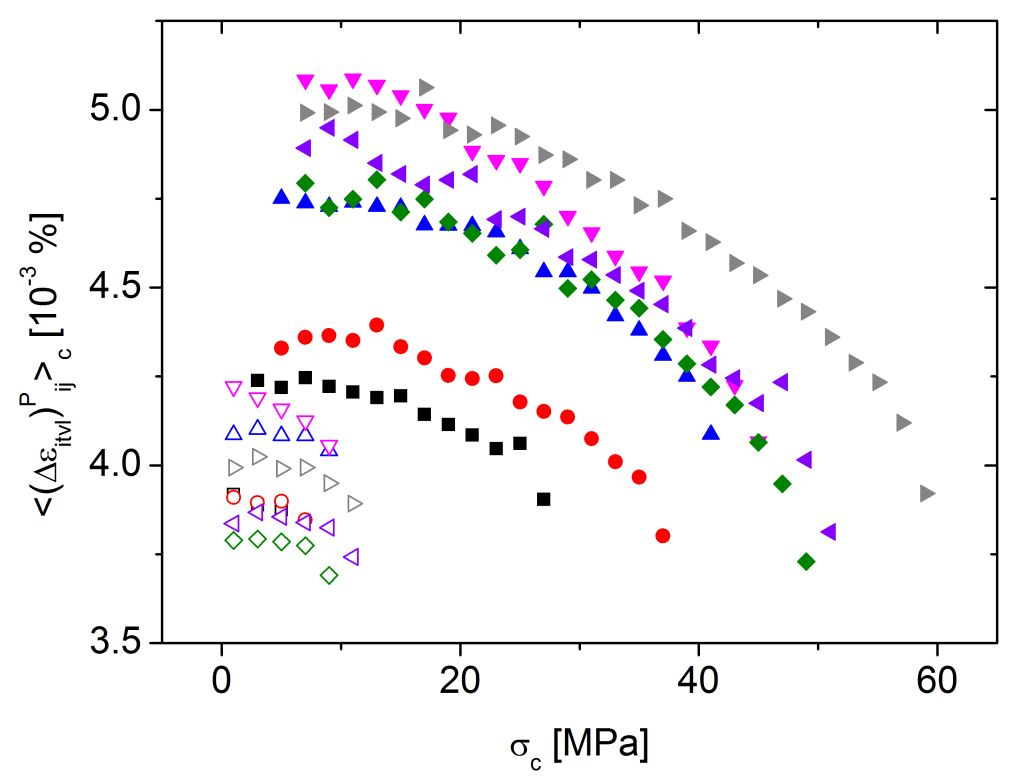

(a) For $\Delta \epsilon_{i t v l}>0$

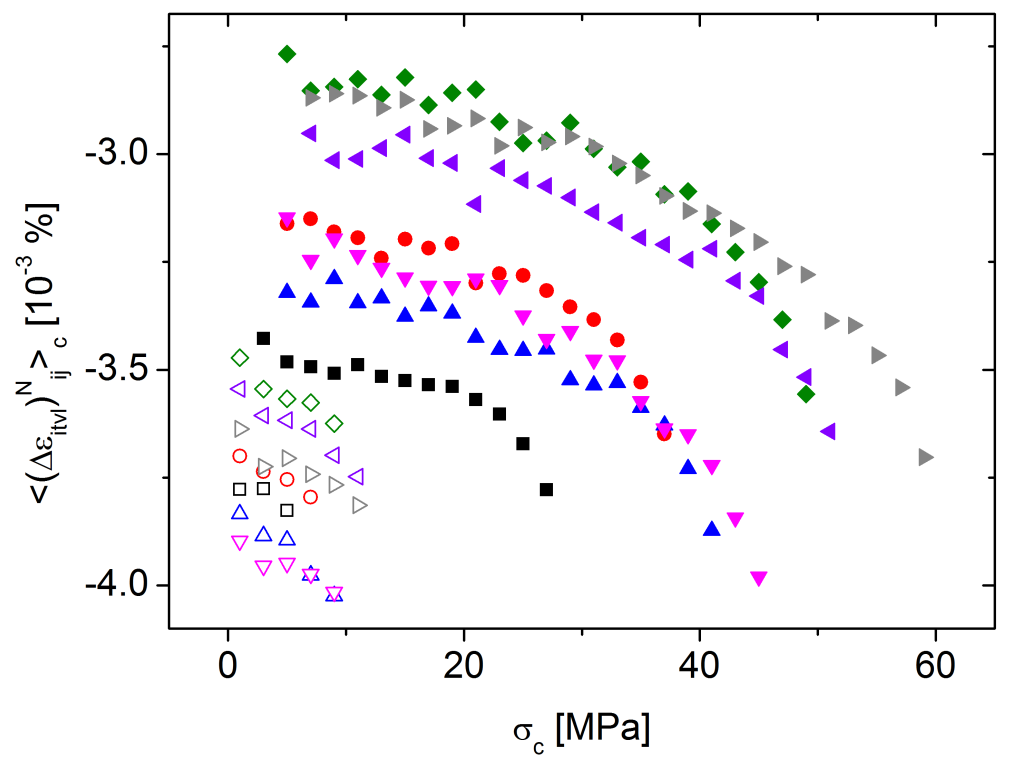

\begin{tabular}{|rr|}
\hline$\square$ & $5.29 \mathrm{MPa}$ \\
$\circ$ & $7.42 \mathrm{MPa}$ \\
$\triangle$ & $8.37 \mathrm{MPa}$ \\
$\nabla$ & $8.85 \mathrm{MPa}$ \\
$\diamond$ & $9.76 \mathrm{MPa}$ \\
$\triangleleft$ & $10.14 \mathrm{MPa}$ \\
$\triangleright$ & $11.82 \mathrm{MPa}$ \\
- & $26.47 \mathrm{MPa}$ \\
$\bullet$ & $37.10 \mathrm{MPa}$ \\
$\triangle$ & $41.85 \mathrm{MPa}$ \\
$\nabla$ & $44.27 \mathrm{MPa}$ \\
$\bullet$ & $48.82 \mathrm{MPa}$ \\
$\triangleleft$ & $50.71 \mathrm{MPa}$ \\
$\triangleright$ & $59.09 \mathrm{MPa}$ \\
\hline
\end{tabular}

(b) For $\Delta \epsilon_{i t v l}<0$

Figure 5.22 Average of interval strain $\left\langle\left(\Delta \epsilon_{i t v l}\right)_{i j}^{L}\right\rangle_{c}$ in steady state for various stress amplitudes $\sigma_{0}$ at constant temperature $T=563 \mathrm{~K}$. (a) for positive and (b) for negative intervals. 
Within a data set, the average strain intervals $\left\langle\left(\Delta \epsilon_{i t v l}\right)_{i j}^{P}\right\rangle_{c}$ yield maximum values very early on the intra-periodic timescale. These maximum values $\left\langle\left(\Delta \epsilon_{i t v l}\right)_{i j}^{P}\right\rangle^{\text {max }}$ show a dominant dependence on the stress amplitude $\sigma_{0}$, as their value increases with increasing $\sigma_{0}$. With increasing values on the $x$-axis, the averaged strain data decreases and is connected to the sinusoidal form of the excitation signal. This becomes evident under normalization of the $x$ - and $y$-axis as the data collapses to a mastercurve (cf.fig. 5.23a). The trajectory of the mastercurve coincides for different temperatures as well. The normalization of the y-axis is accomplished by subtraction of and division by the maximum average strain of the data set, concluding with the division by the minimum value of the data set according to equation 5.1 for $\iota=P$.

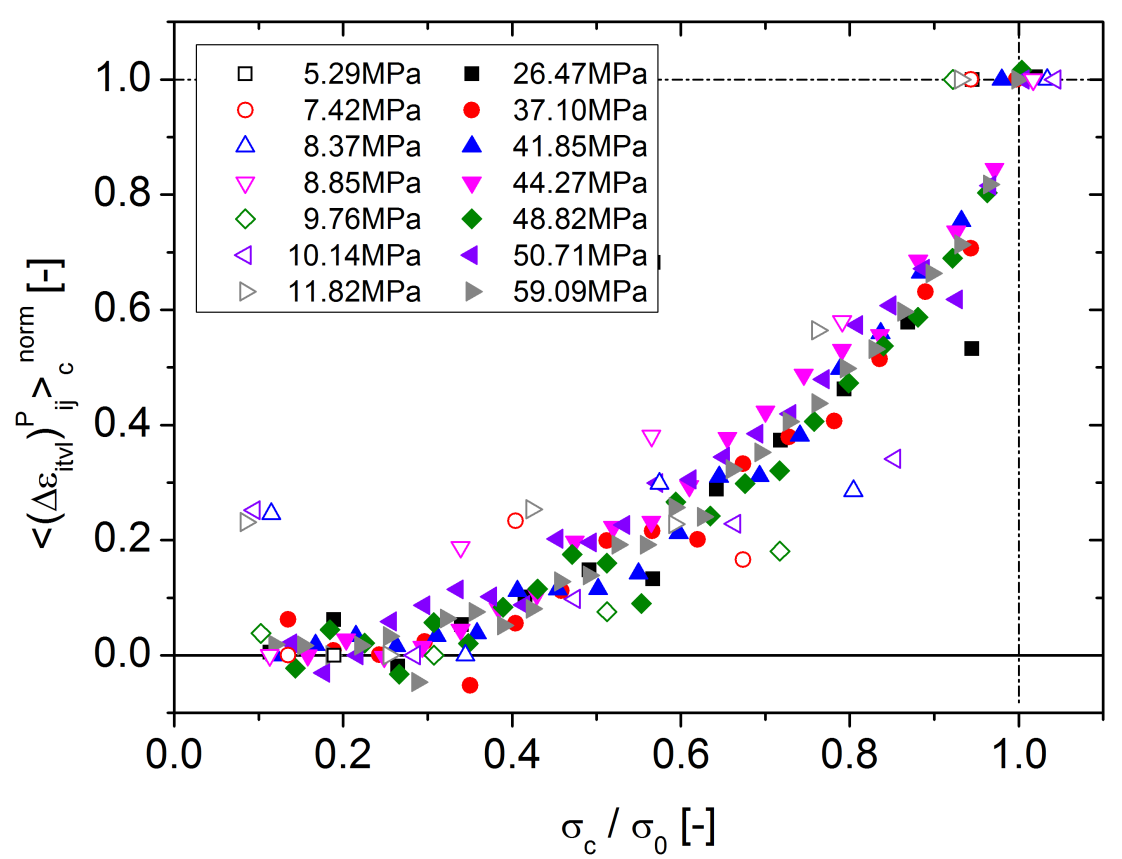

(a) For $\Delta \epsilon_{i t v l}>0$

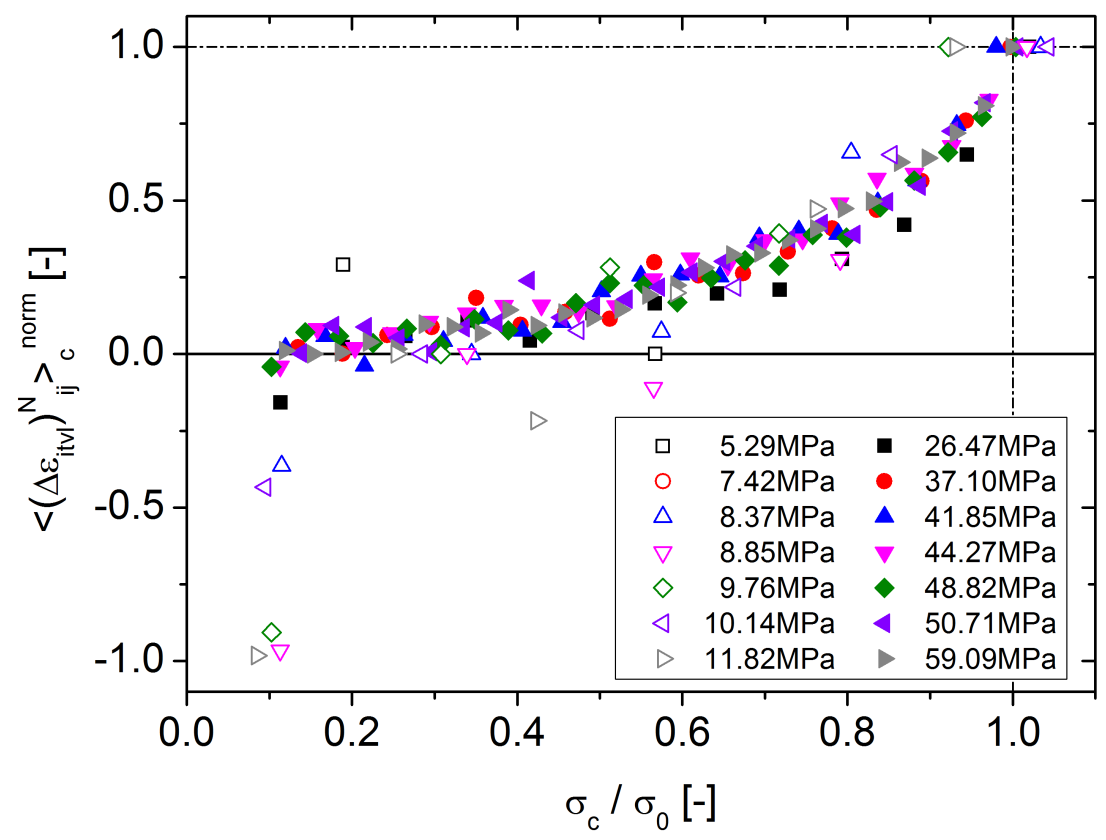

(b) For $\Delta \epsilon_{i t v l}<0$

Figure 5.23 Normalized average of interval strain $\left\langle\left(\Delta \epsilon_{i t v l}\right)_{i j}^{\iota}\right\rangle_{c}^{\text {norm }}$ for various stress amplitudes $\sigma_{0}$ at constant temperature $T=563 \mathrm{~K}$. (a) for positive and (b) for negative intervals. 


$$
<\left(\Delta \epsilon_{i t v l}\right)_{i j}^{L}>_{c}^{\text {norm }}=\left(\frac{\left.\left\langle\left(\Delta \epsilon_{i t v l}\right)_{i j}^{L}\right\rangle_{c}-<\left(\Delta \epsilon_{i t v l}\right)_{i j}^{L}\right\rangle^{\max }}{<\left(\Delta \epsilon_{i t v l}\right)_{i j}^{L}>^{\max }}\right) /<\left(\Delta \epsilon_{i t v l}\right)_{i j}^{L}>^{\min }
$$

The dependence on stress for the average interval strain $\left\langle\left(\Delta \epsilon_{i t v l}\right)_{i j}^{N}\right\rangle_{c}$ in case of negative intervals is depicted in fig. 5.22b. The data sets are similarly related to the stress amplitude as in case of positive intervals. The higher the stress amplitude $\sigma_{0}$ is set, the less negative the average strain values become. The data can be normalized to collapse to a mastercurve as described in the case of positive interval averages. Also for negative intervals, the trajectories of data sets measured at different temperatures and stresses fall upon another in respect to the accuracy of the measurements.

Moreover, both the mastercurves for positive and negative intervals of averaged interval strain coincide with the trajectory of the mastercurve for the strain rate (cf. fig. 5.20b).

If $\left\langle\left(\Delta \epsilon_{i t v l}\right)_{i j}^{L}\right\rangle^{\max }$ and $\left\langle\left(\Delta \epsilon_{i t v l}\right)_{i j}^{L}\right\rangle^{\min }$, which are used for the normalization of averaged strain values, are plotted against the stress amplitude as in fig. 5.24. it becomes obvious that the maximum average strain depends linearly on $\sigma_{0}$. A linear regression on $\left\langle\left(\Delta \epsilon_{i t v l}\right)_{i j}^{P}\right\rangle^{\max }$ yields a slope of $2.4(2) \times 10^{-5} \% \mathrm{MPa}^{-1}$ (dashed line in fig. 5.24a) and an ordinate value of $3.7(1) \times 10^{-3} \%$.

For $\left\langle\left(\Delta \epsilon_{i t v l}\right)_{i j}^{N}\right\rangle^{\max }$ the linear regression gives a slope of $1.5(2) \times 10^{-5} \% \mathrm{MPa}^{-1}$ and an ordinate value of $-3.8(1) \times 10^{-3} \%$.

The minimum $<\left(\Delta \epsilon_{i t v l}\right)_{i j}^{L}>^{\min }$ shows constant behavior with a mean value of $3.8(3) \times 10^{-3} \%$ in case of positive strain and $-3.8(2) \times 10^{-3} \%$ for negative strain averages. These mean values coincide with the ordinates determined from linear regressions on $\left\langle\left(\Delta \epsilon_{i t v l}\right)_{i j}^{L}>^{\max }\right.$ within the limits of errors.

This reflects, that the stress amplitude influences the averaged strain mainly on the initial part of the intra-periodic timescale, where the averaged strain shows a maximum at $<\left(\Delta \epsilon_{i t v l}\right)_{i j}^{\iota}>^{\max }$ both for positive $(\iota=P)$ and negative $(\iota=N)$ intervals. 


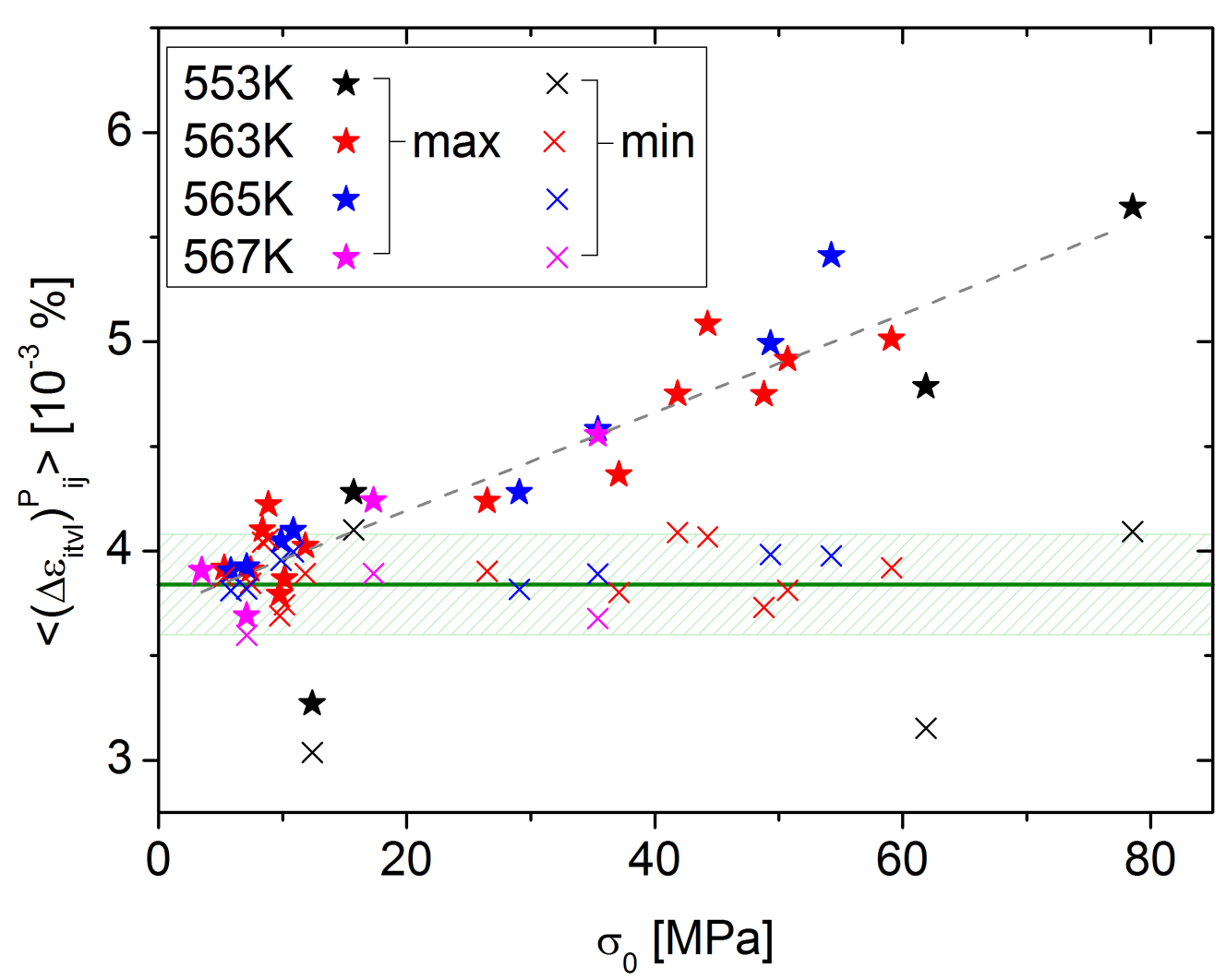

(a) For $\Delta \epsilon_{i t v l}>0$

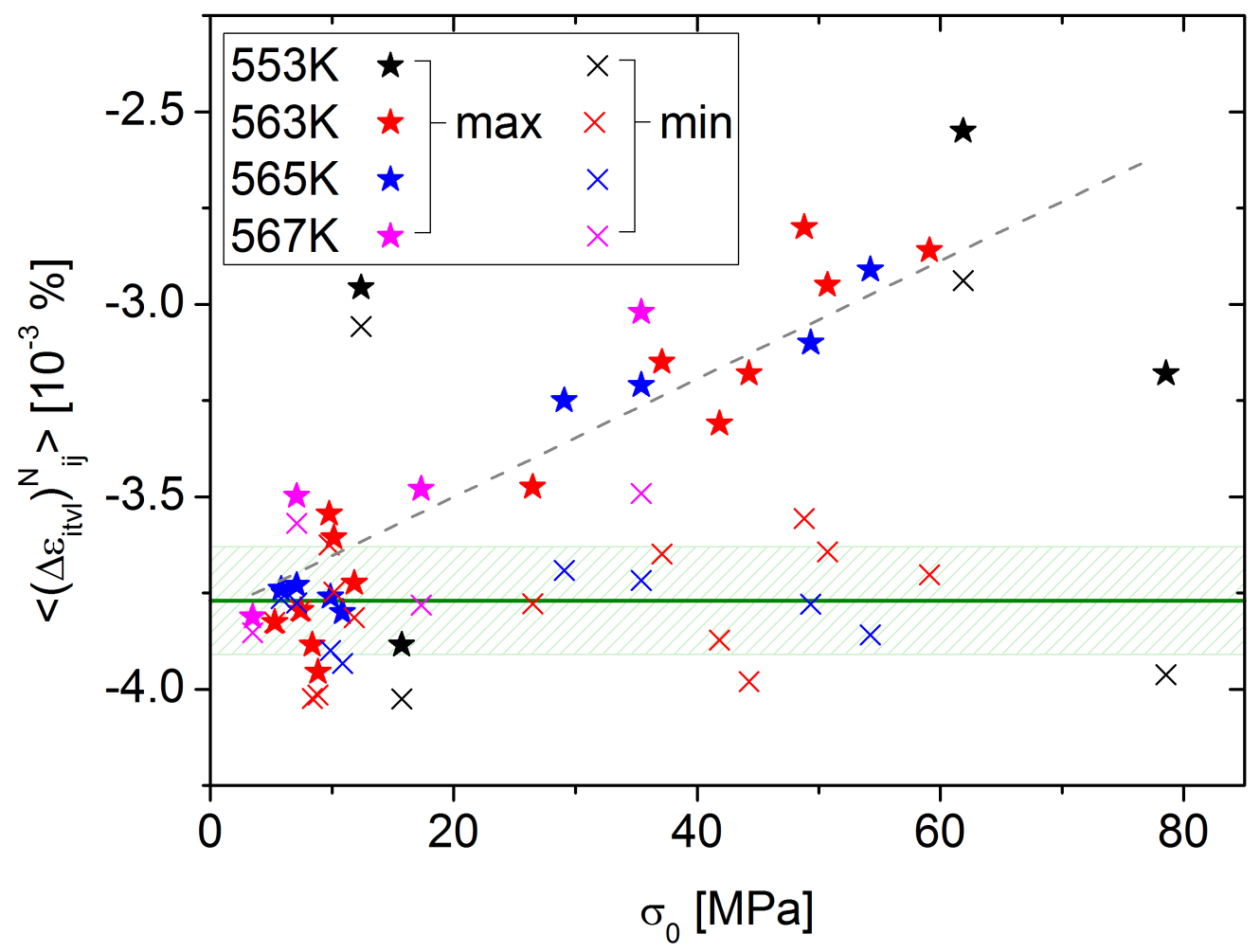

(b) For $\Delta \epsilon_{i t v l}<0$

Figure 5.24 Minimum (min) and maximum (max) values of interval strain, $\left\langle\left(\Delta \epsilon_{i t v l}\right)_{i j}^{L}>^{\min }\right.$ and $<\left(\Delta \epsilon_{i t v l}\right)_{i j}^{L}>^{\max }$,for various stress amplitudes $\sigma_{0}$ and temperatures $T$. (a) for positive and (b) for negative intervals. Green lines correspond to the mean value of $\left\langle\left(\Delta \epsilon_{i t v l}\right)_{i j}^{\iota}\right\rangle^{\min }$, while the shaded area reflects the standard deviation. 


\section{Averages of Interval Duration}

Averaged data on interval duration for positive intervals are plotted against intra-periodic stress $\sigma_{c}$ in fig. 5.25a. Fig. 5.25b depicts this quantity for negative intervals. Shown are in either case the mean values for low field (open symbols) and high field (solid symbols) and the according standard deviations (error bars) measured at a temperature of $563 \mathrm{~K}$. Intervals of both signs, positive and negative, show very similar behavior. Even though the error bars exceed in all cases the difference between the mean values, an increase of the averaged durations with $\sigma_{c}$ is generally observed. As the case for interval strain, the interval duration does not seem to depend on the absolute value of stress, as it decreases with rising stress amplitude and shows the opposite with increasing intra-periodic stress.

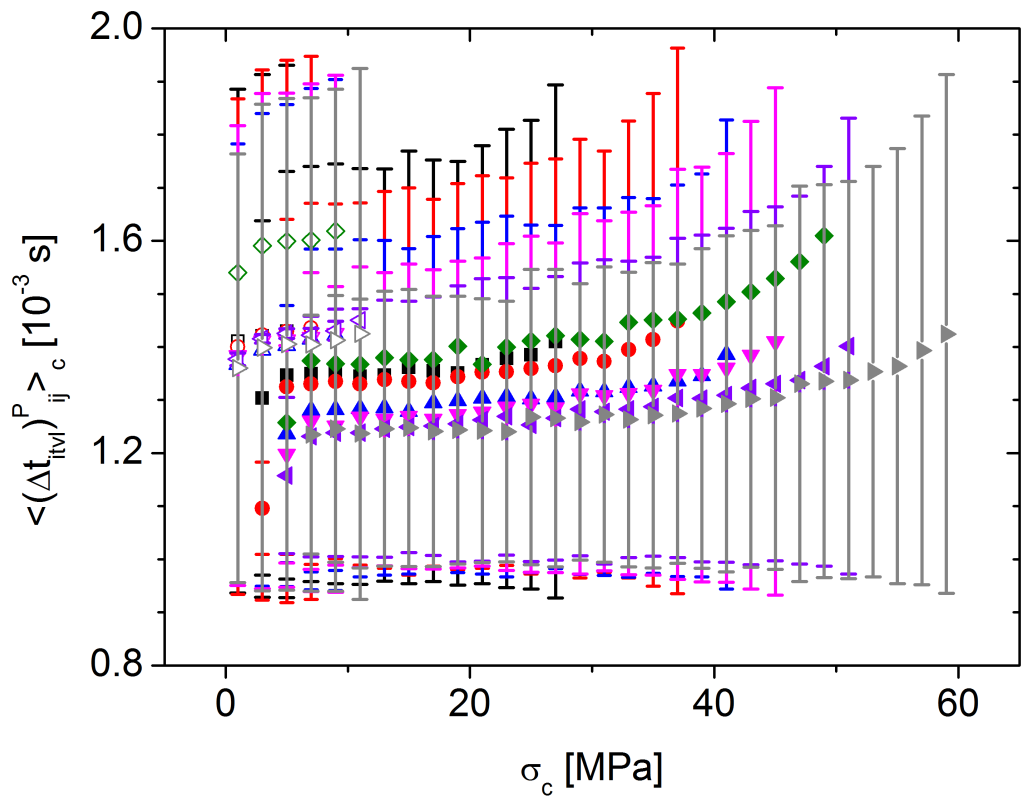

(a) For $\Delta \epsilon_{i t v l}>0$

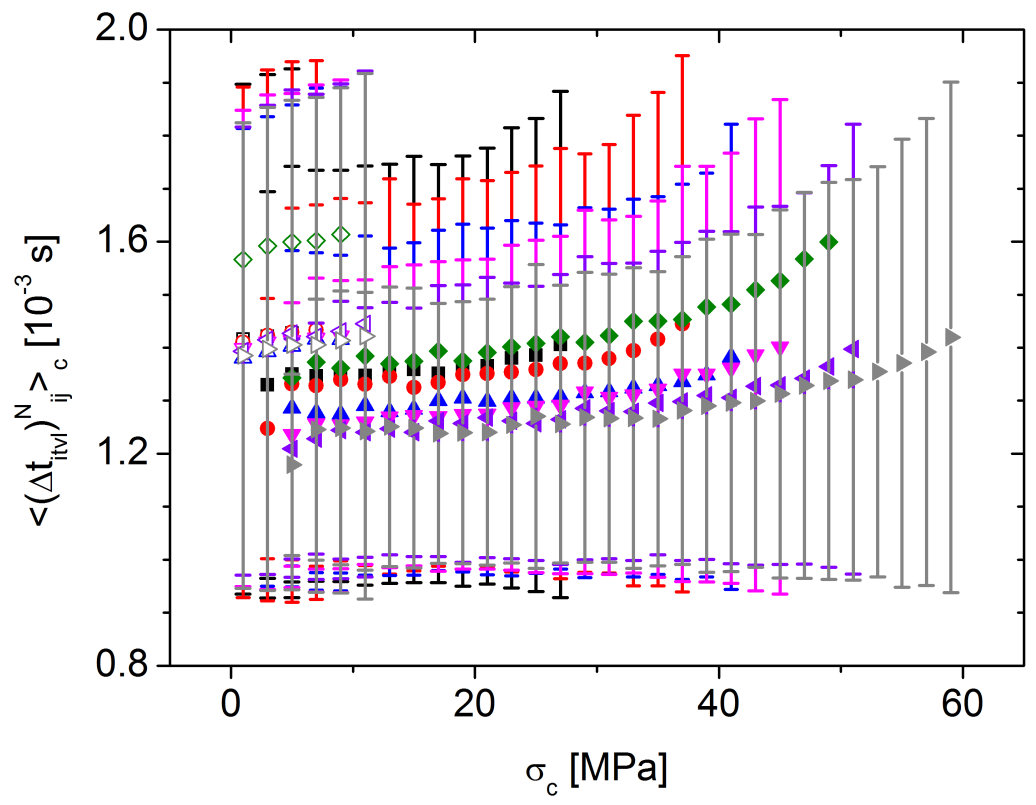

\begin{tabular}{|rr|}
\hline$\square$ & $5.29 \mathrm{MPa}$ \\
$\circ$ & $7.42 \mathrm{MPa}$ \\
$\triangle$ & $8.37 \mathrm{MPa}$ \\
$\nabla$ & $8.85 \mathrm{MPa}$ \\
$\diamond$ & $9.76 \mathrm{MPa}$ \\
$\triangleleft$ & $10.14 \mathrm{MPa}$ \\
$\triangleright$ & $11.82 \mathrm{MPa}$ \\
- & $26.47 \mathrm{MPa}$ \\
$\bullet$ & $37.10 \mathrm{MPa}$ \\
$\triangle$ & $41.85 \mathrm{MPa}$ \\
$\nabla$ & $44.27 \mathrm{MPa}$ \\
$\bullet$ & $48.82 \mathrm{MPa}$ \\
$\triangleleft$ & $50.71 \mathrm{MPa}$ \\
$\triangleright$ & $59.09 \mathrm{MPa}$ \\
\hline
\end{tabular}

(b) For $\Delta \epsilon_{i t v l}<0$

Figure 5.25 Averages of interval duration $\left\langle\left(\Delta t_{i t v l}\right)_{i j}\right\rangle_{c}$ in steady state for various stress amplitudes $\sigma_{0}$ at constant temperature $T=563 \mathrm{~K}$. (a) for positive and (b) for negative intervals. 
A normalization of $\sigma_{c}$ by $\sigma_{0}$ on the x-axis in connection to a normalization of the $y$-axis according to eq. 5.2 results in a collapse of the different data sets onto a mastercurve as in fig. 5.26 .

$$
<\left(\Delta t_{i t v l}\right)_{i j}^{\iota}>_{c}^{n_{c}}=\left(\frac{\left\langle\left(\Delta t_{i t v l}\right)_{i j}^{L}>_{c}-<\left(\Delta t_{i t v l}\right)_{i j}^{L}>^{\max }\right.}{<\left(\Delta t_{i t v l}\right)_{i j}^{L}>^{\max }}\right) /<\left(\Delta t_{i t v l}\right)_{i j}^{L}>^{\min } .
$$

Here, $\left\langle\left(\Delta t_{i t v l}\right)_{i j}^{\iota}\right\rangle^{\max }$ denotes the maximum average value of a data set, which is located at the highest intra-periodic stress. $\left\langle\left(\Delta t_{i t v l}\right)_{i j}^{L}>^{\text {min }}\right.$ reflects the plateau-like regime at low, even though not generally lowest $\sigma_{c}$.

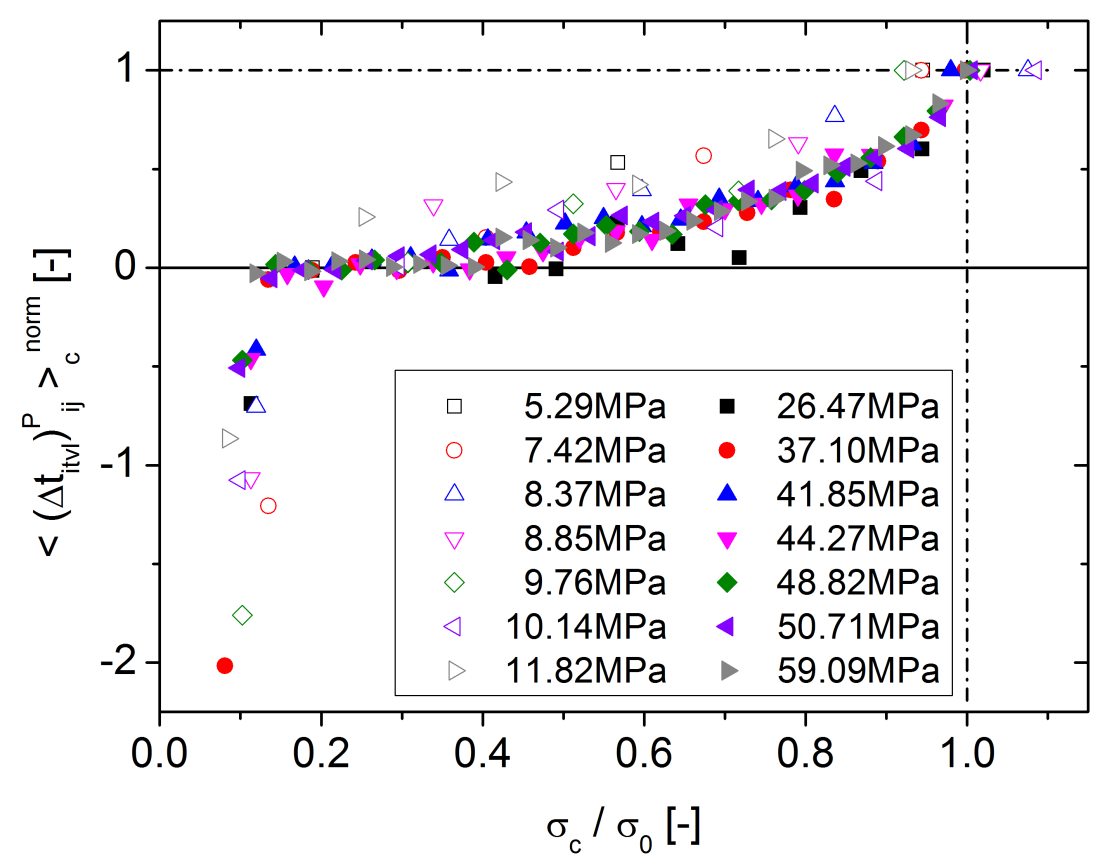

(a) For $\Delta \epsilon_{i t v l}>0$

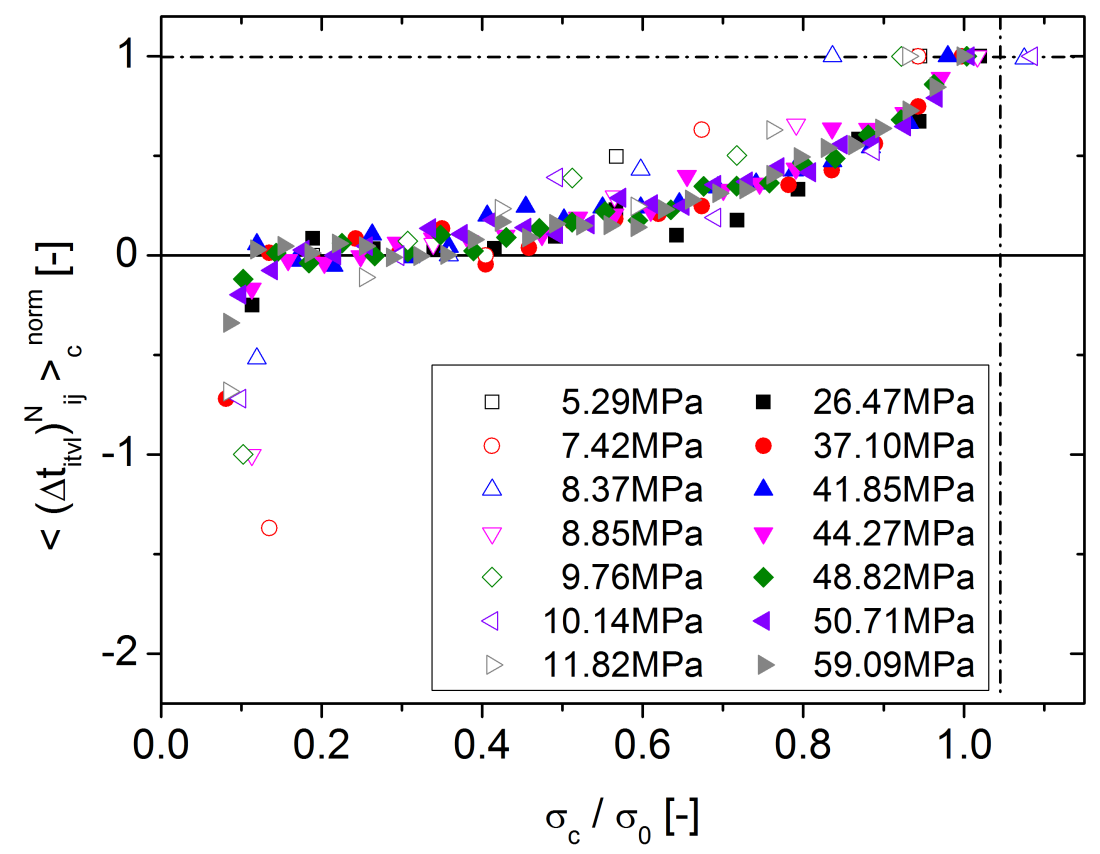

(b) For $\Delta \epsilon_{i t v l}<0$

Figure 5.26 Normalized average interval duration $\left\langle\left(\Delta t_{i t v 1}\right)_{i j}^{\iota}>_{c}^{\text {norm }}\right.$ for various stress amplitudes $\sigma_{0}$ at constant temperature $T=563 \mathrm{~K}$. (a) for positive and (b) for negative intervals. 


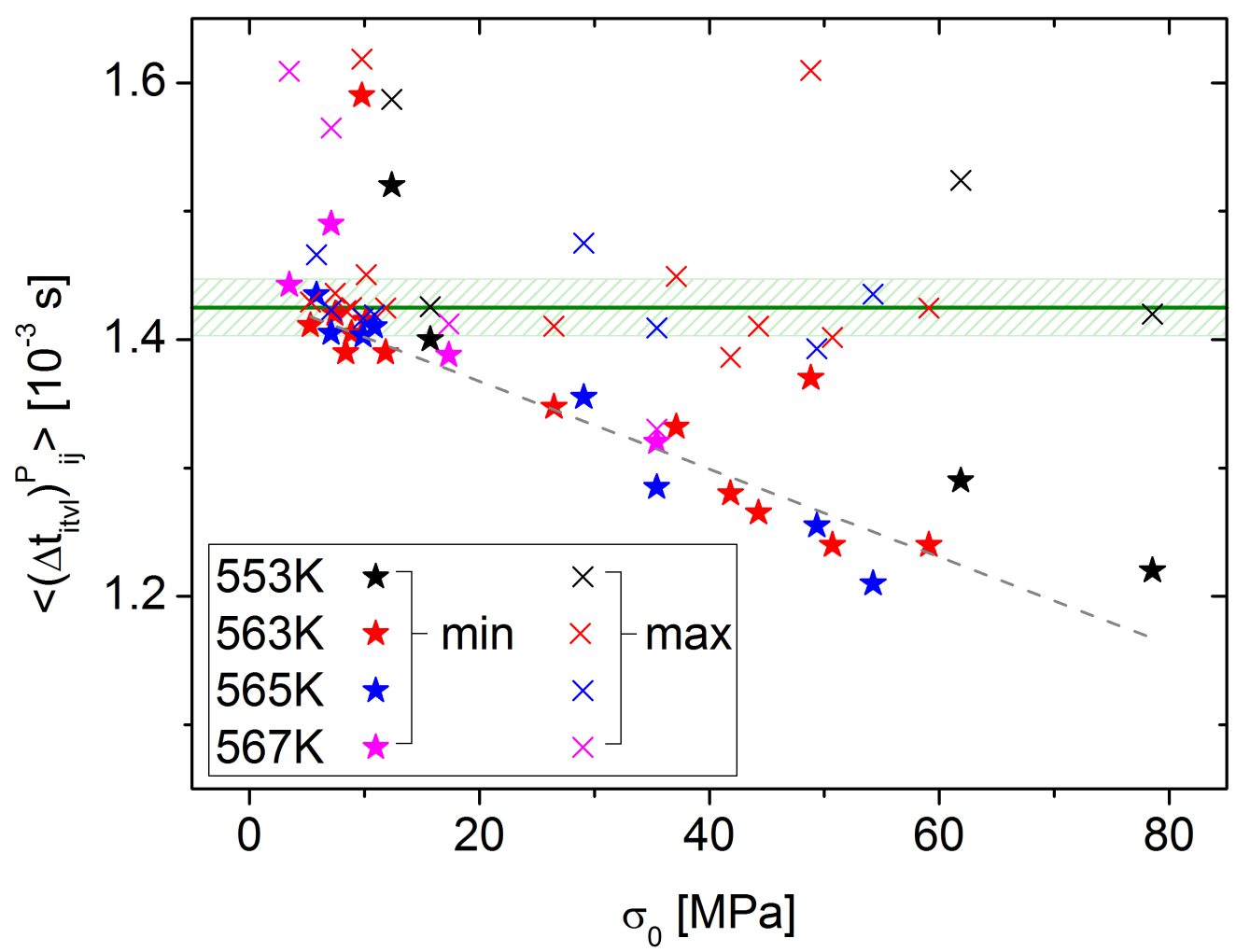

(a) For $\Delta \epsilon_{i t v l}>0$

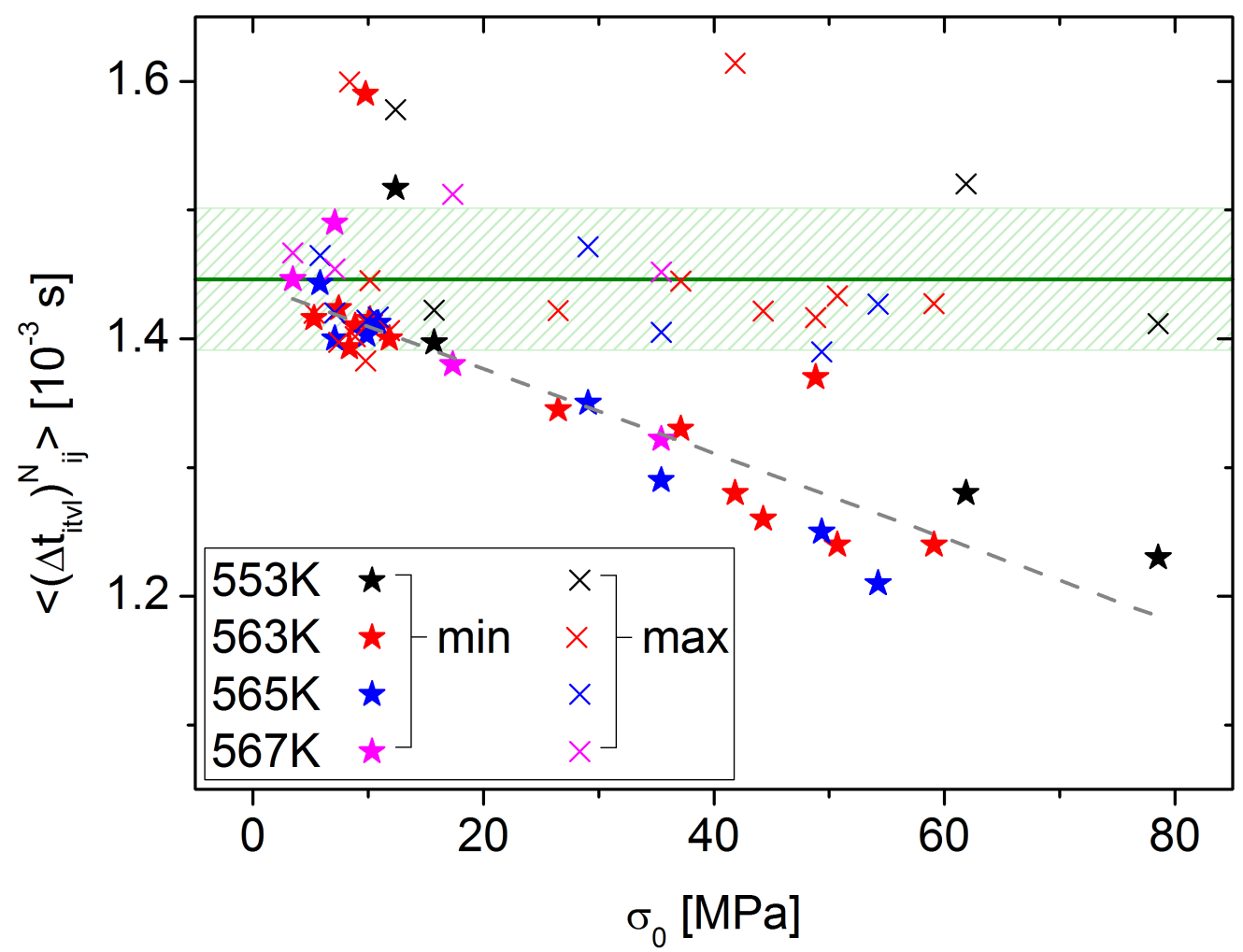

(b) For $\Delta \epsilon_{i t v l}<0$

Figure 5.27 Minimum and maximum values of interval duration, $<\left(\Delta t_{i t v l}\right)_{i j}^{\iota}>^{\min }$ and $<$ $\left(\Delta t_{i t v l}\right)_{i j}^{l}>^{\max }$, for various stress amplitudes $\sigma_{0}$ and temperatures $T$. (a) for positive and (b) for negative intervals. Green lines correspond to the mean value of $\left\langle\left(\Delta \epsilon_{i t v l}\right)_{i j}^{L}\right\rangle^{\min }$, while the shaded area reflects the standard deviation. 
The values at low $\sigma_{c},\left\langle\left(\Delta t_{i t v l}\right)_{i j}^{L}\right\rangle^{\text {min }}$, extracted from fig 5.25 show a clear dependence on $\sigma_{0}$ as a decrease is observed with increasing stress amplitude. This behavior was extrapolated by a linear regression, yielding a slope of $-3.4(3) \times 10^{-3} \mathrm{~ms} \mathrm{MPa}^{-1}$ and an ordinate value of $1.4(1) \mathrm{ms}$ in case of positive intervals. For negative intervals, the slope and ordinate yield identical values in the range of the error. The data on the maxima of interval durations show a constant behavior and are thus independent of $\sigma_{0}$. Both for positive and negative intervals a mean value of $1.4(1) \mathrm{ms}$ is measured including data of different temperatures. The values extracted for the mean and the ordinate are equal within the regime of the errors, also if data for positive and negative intervals are compared. Thus, the dependence of $\left\langle\Delta t_{i t v l}\right\rangle$ on the stress amplitude $\sigma_{0}$ and the intra-periodic stress $\sigma_{c}$ is on average the same for positive and negative intervals. 


\subsubsection{Distributions of Strain Intervals}

The influence of temperature $T$ and stress amplitude $\sigma_{0}$ on the distribution of positive and negative strain intervals $\left(\Delta \epsilon_{i t v l}\right)^{l}$ is analyzed. Therefore the value of strain intervals and their occurrence are plotted in a double-logarithmic scaling. The values included in the distributions correspond to the initial 2/3 of the amplitude of the positive ascending parts of steady state periods, i.e. all data points at intra-period stresses which are below the green dotted line as exemplified in fig. 3.6. Data sets include steady state periods which are either based on the initial low field or the high field response as defined before.

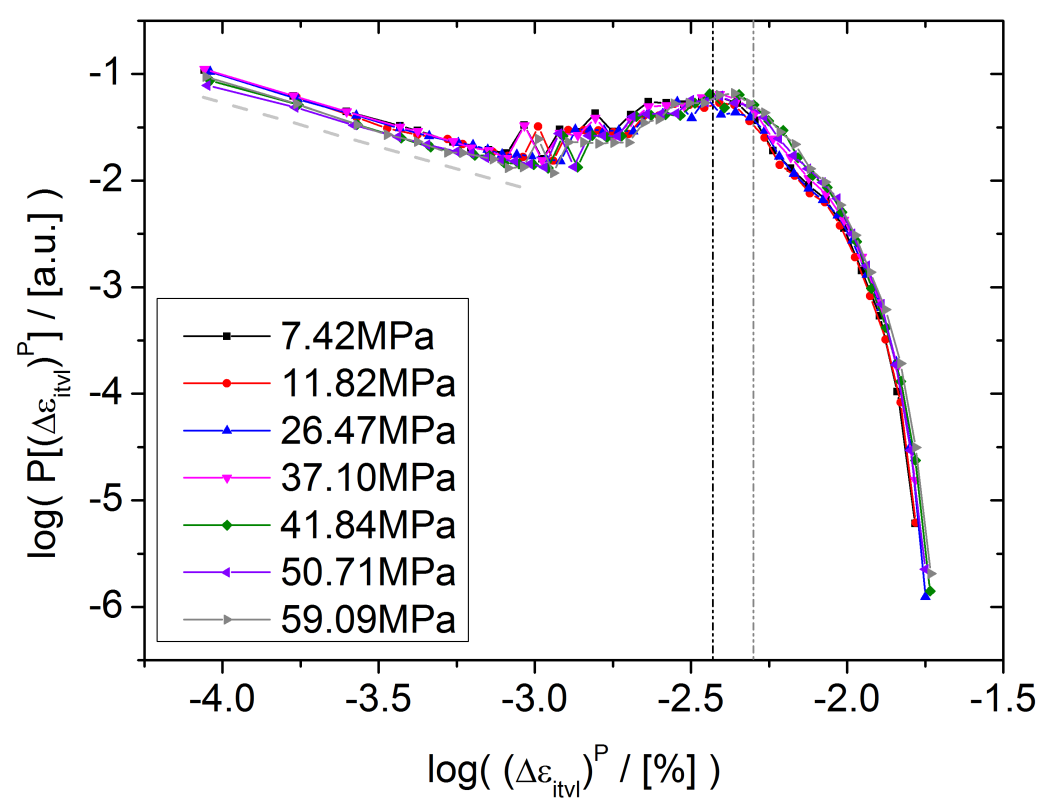

(a) For $\Delta \epsilon_{i t v l}>0$

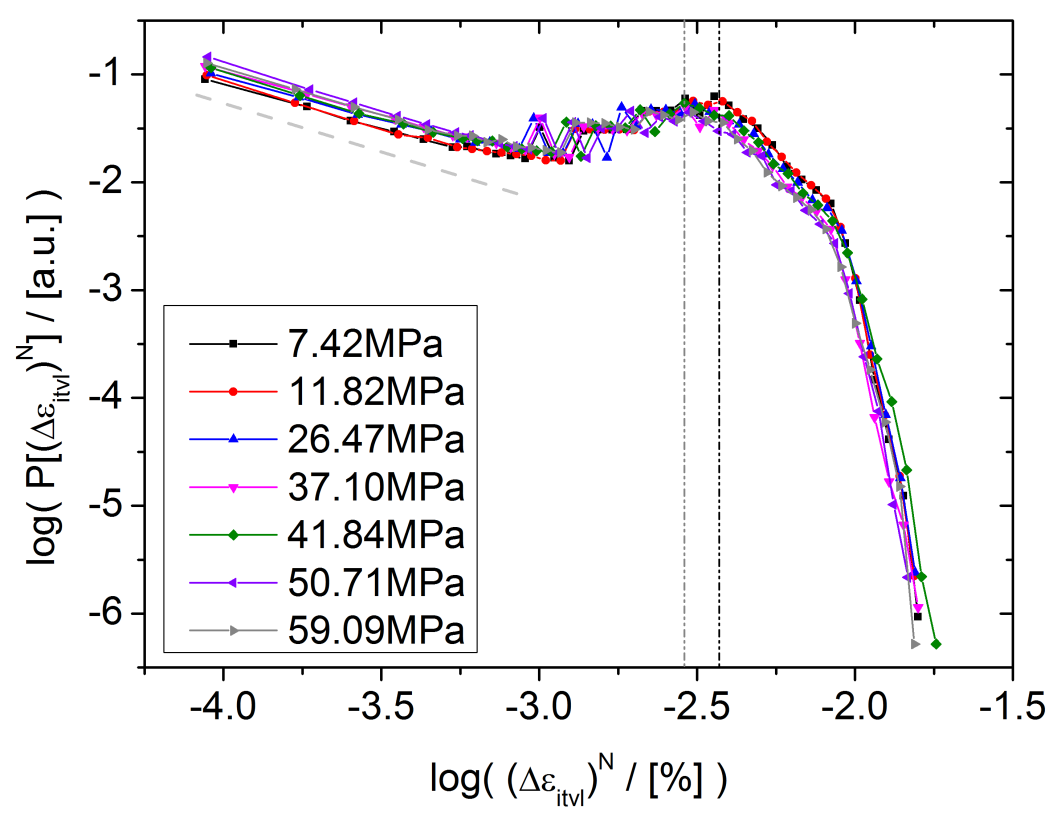

(b) For $\Delta \epsilon_{i t v l}<0$

Figure 5.28 Double-logarithmic distribution of interval strain $\left(\Delta \epsilon_{i t v l}\right)^{\iota}$ for various stress amplitudes $\sigma_{0}$ at a constant temperature $T$ of $563 \mathrm{~K}$. Dashed-dotted lines correspond to maximum average strain values for measurements with a stress amplitude of $7 \mathrm{MPa}$ (black) and of $59 \mathrm{MPa}$ (gray). The dashed light gray line indicates the power-law regime. (a) for positive and (b) for negative intervals. 
Fig. 5.28a and 5.28b show distributions for positive and negative strain intervals at $563 \mathrm{~K}$ for several stress amplitudes. The distributions have the following features in common, which are qualitatively comparable for positive and negative interval data, and independent of temperature $T$ or stress amplitude $\sigma_{0}$.

The distributions show a decreasing slope at low strain values, that passes into a hump and is followed by a cut-off. The range of the initial decreasing linear part and the onset of the hump are strongly influenced by the number of bins, while the slope itself is not influenced by the binning. From the linearly decreasing part of the distribution at very small values, the slope is extracted by linear regression. It is evaluated in regard to its dependence on temperature and stress amplitude.

Distributions of positive and negative strain intervals at low stress amplitude exhibit very similar profiles. Compared to the high field steady state distributions, minor changes can be spotted, which are in accordance with the analysis of the averaged strain interval data. In the regime of the hump, the distribution of values for positive strain intervals $\left(\Delta \epsilon_{i t v l}\right)^{P}$ shifts towards higher strain values with increasing stress amplitude. The hump of the distribution of negative strain intervals $\left(\Delta \epsilon_{i t v l}\right)^{N}$ on the contrary shifts towards smaller values with increasing stress amplitude.

The analysis of power-law behavior is conducted in the range of small strain interval data corresponding to the initial part of the distribution. The range of the linear behavior in the log-log-representation extends over roughly one order of magnitude. The exponent denoted as $\kappa$ is extracted for each data set by linear regression over the regime of interest, what is indicated by the dashed light gray line in fig. 5.29 and 5.30 . The values of $\kappa$ are plotted against the stress amplitude $\sigma_{0}$ for various temperatures, for positive strain intervals in fig. 5.31a and for negative strain values in fig. 5.31b. The error bar on the data points is due to the error of the slope $\kappa$ extracted from the linear fits. In both figures, the exponent $\kappa$ does not show a dependence on stress amplitude or temperature and yields a mean value and standard deviation of $-0.75(8)$ in case of positive and $-0.75(15)$ in case of negative strain intervals. In the plot, the mean is depicted as a dashed line, the shaded area reflects the standard deviation.

If the distributions of fig. 5.29 are taken into account, the temperature seems to have a suppressing behavior on the hump. In comparison, the behavior at higher stress amplitudes as in fig. 5.30 does not show a clear influence of temperature. However, the distribution on negative intervals based on data measured at $553 \mathrm{~K}$ and a stress amplitude of $60 \mathrm{MPa}$ shows a power-law behavior over the full regime of measured interval strain (cf. fig. 5.30b). The hump is not observed, and the exponent extracted over interval strain values spanning two decades yields a value of $-0.78(4)$. It is not evident which parameters trigger the suppression of the hump. This behavior is only observed in case of the evaluation of the negative intervals occurring during the high-field excitation of the LAOS-measurement. The distributions on the according low field excitation (fig. 5.29b) and the distribution on the positive intervals (fig. 5.30a) exhibit a hump. 


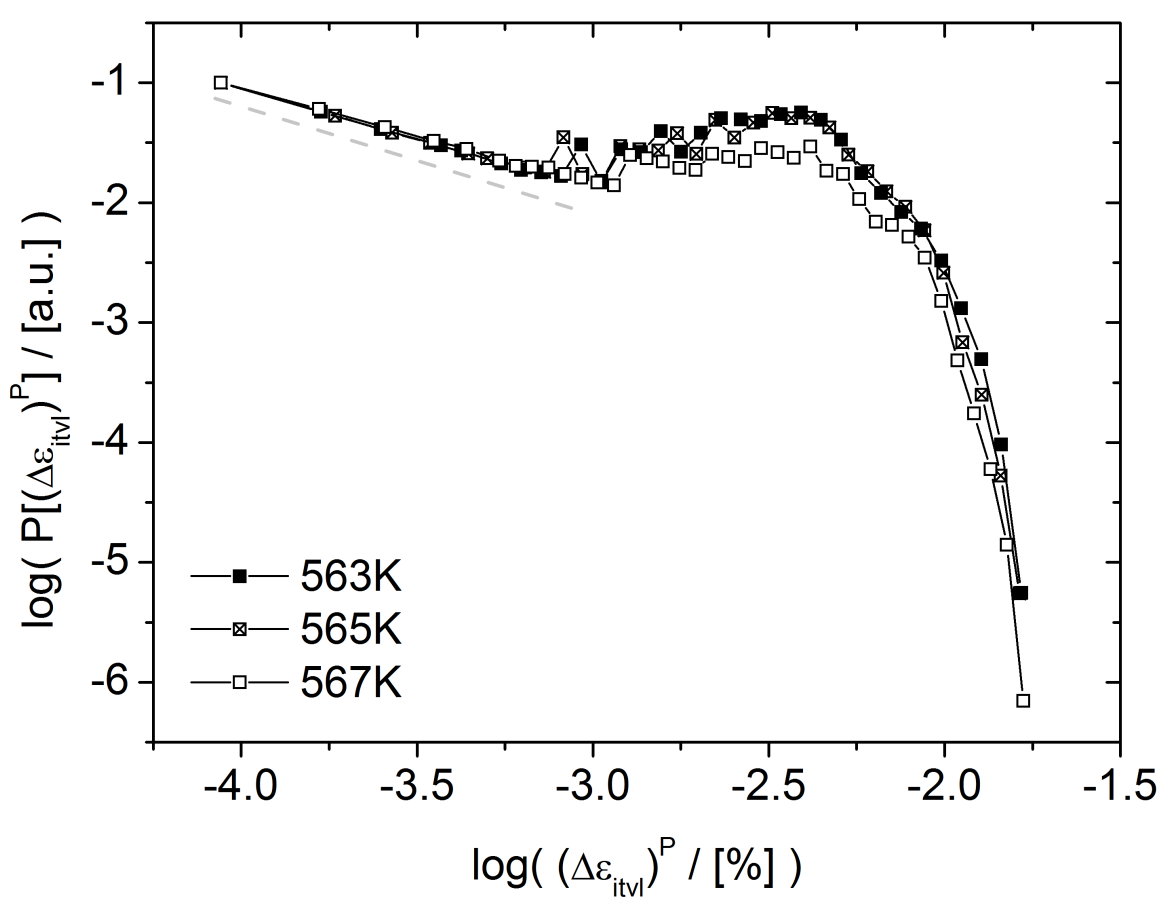

(a) For $\Delta \epsilon_{i t v l}>0$

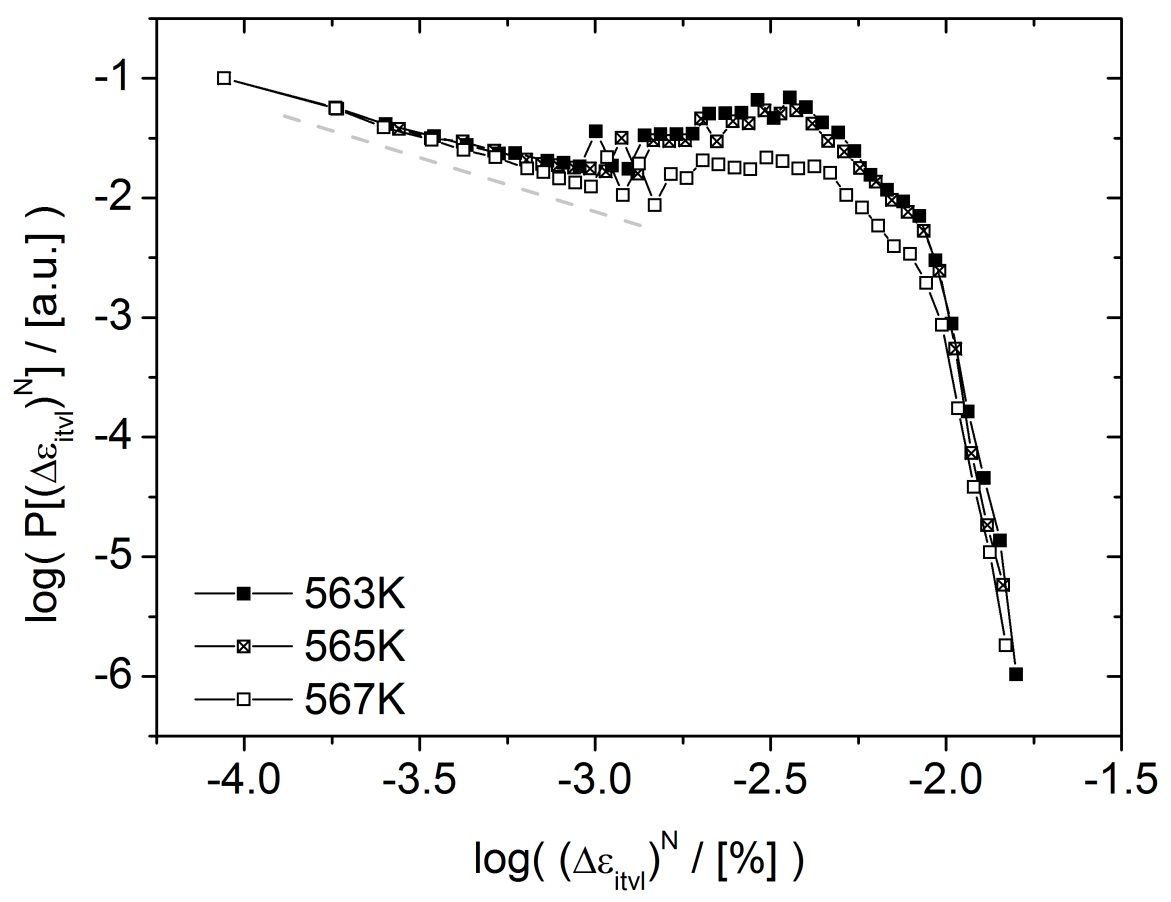

(b) For $\Delta \epsilon_{i t v l}<0$

Figure 5.29 Double-logarithmic distributions of interval strain $\left(\Delta \epsilon_{i t v /}\right)^{\iota}$ at a stress amplitude $\sigma_{0}$ of $7 \mathrm{MPa}$ comparing various temperatures $T$. The dashed light gray line indicates the power-law regime. (a) for positive and (b) for negative intervals. 


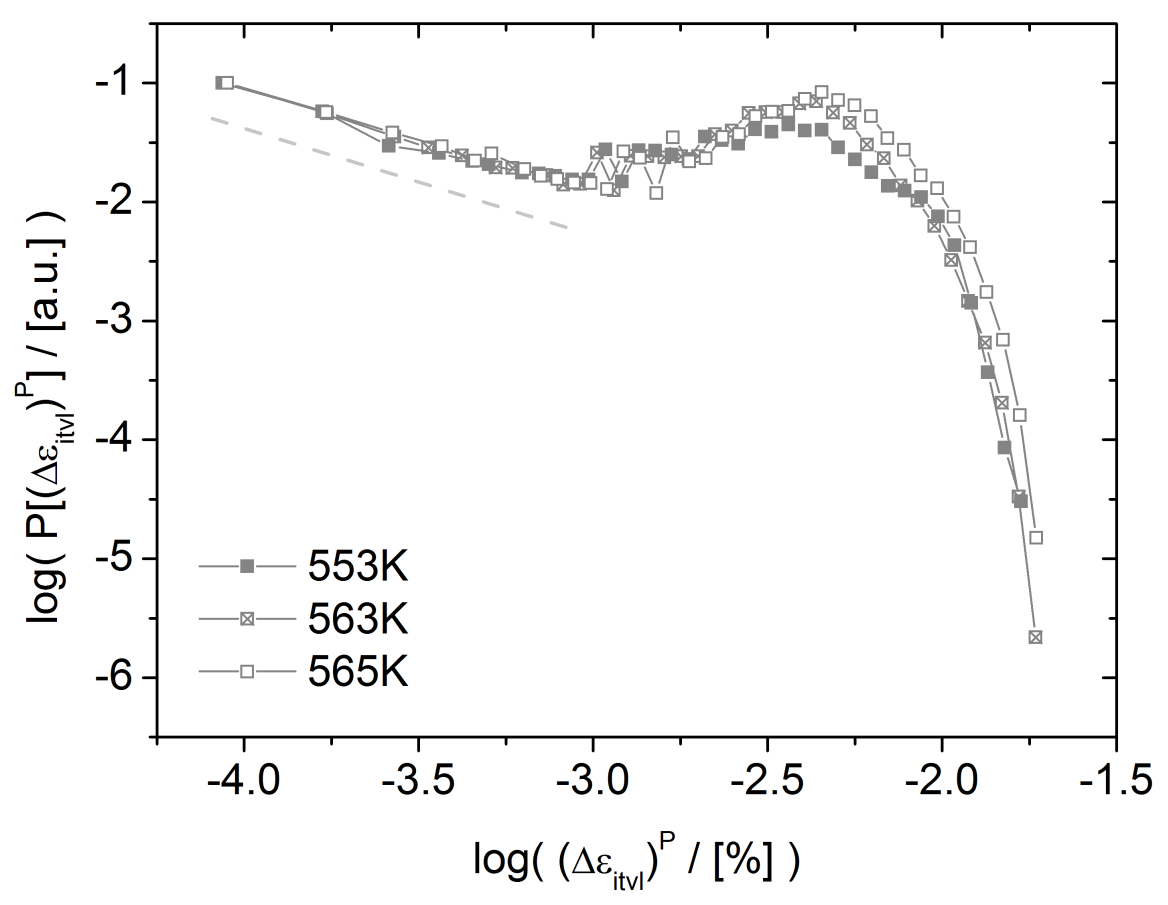

(a) For $\Delta \epsilon_{i t v l}>0$

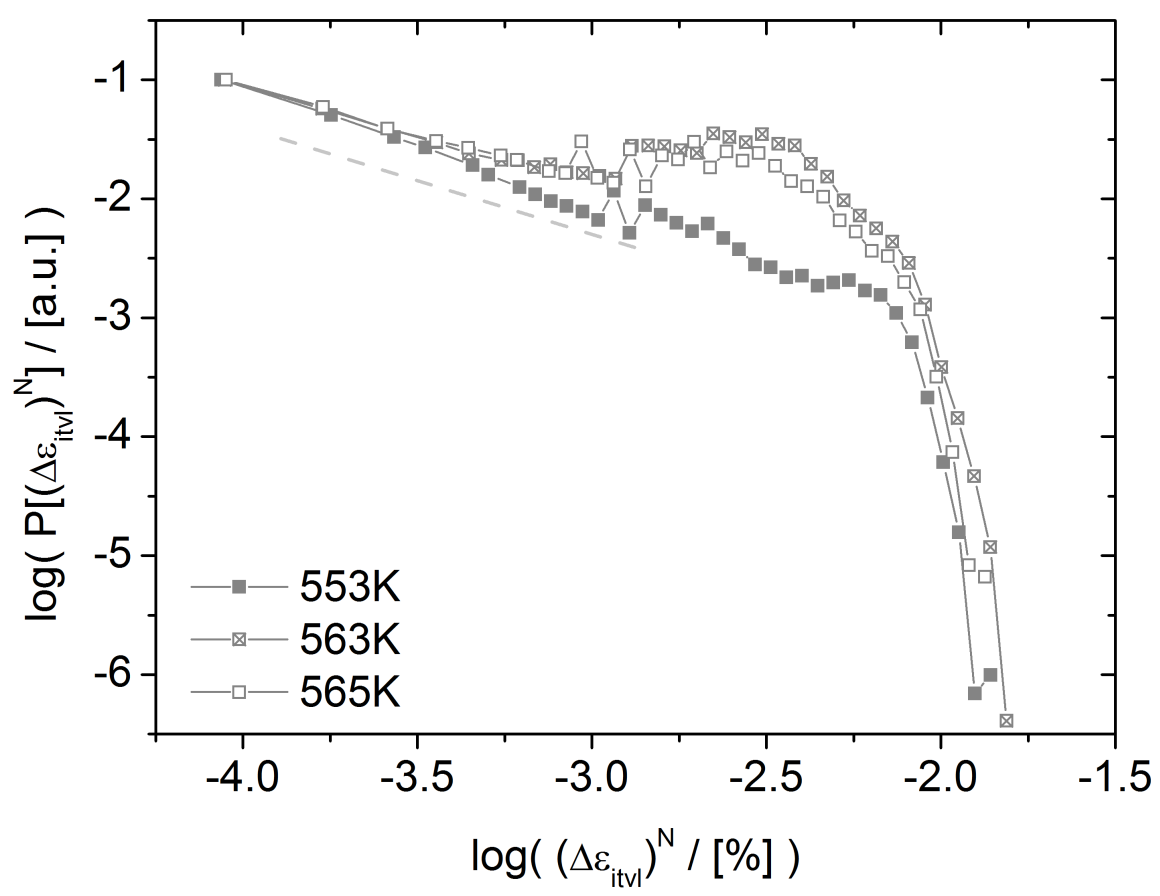

(b) For $\Delta \epsilon_{i t v l}<0$

Figure 5.30 Double-logarithmic distributions of interval strain $\left(\Delta \epsilon_{i t v l}\right)^{\iota}$ at a stress amplitude $\sigma_{0}$ of $60 \mathrm{MPa}$ comparing various temperatures $T$. The dashed light gray line indicates the power-law regime. (a) for positive and (b) for negative intervals. 


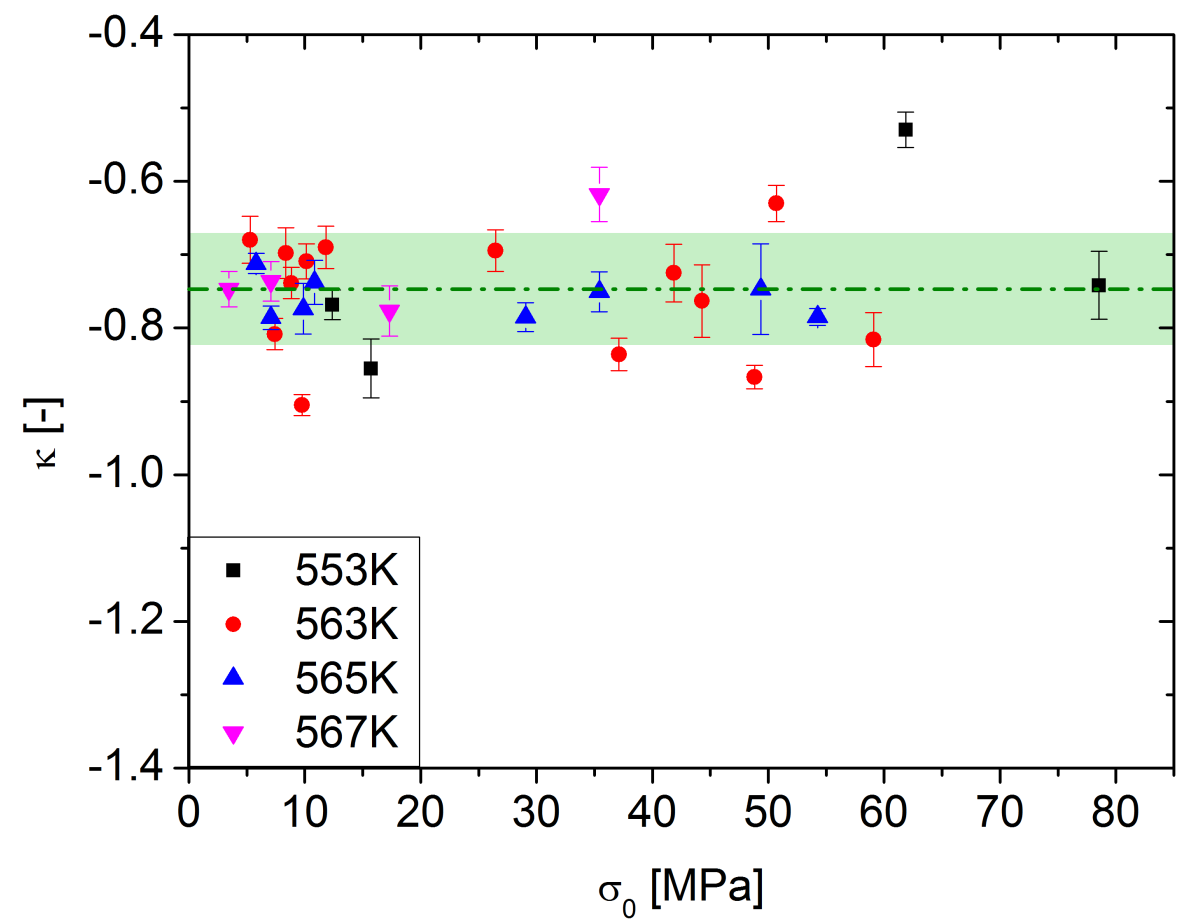

(a) For $\Delta \epsilon_{i t v l}>0$

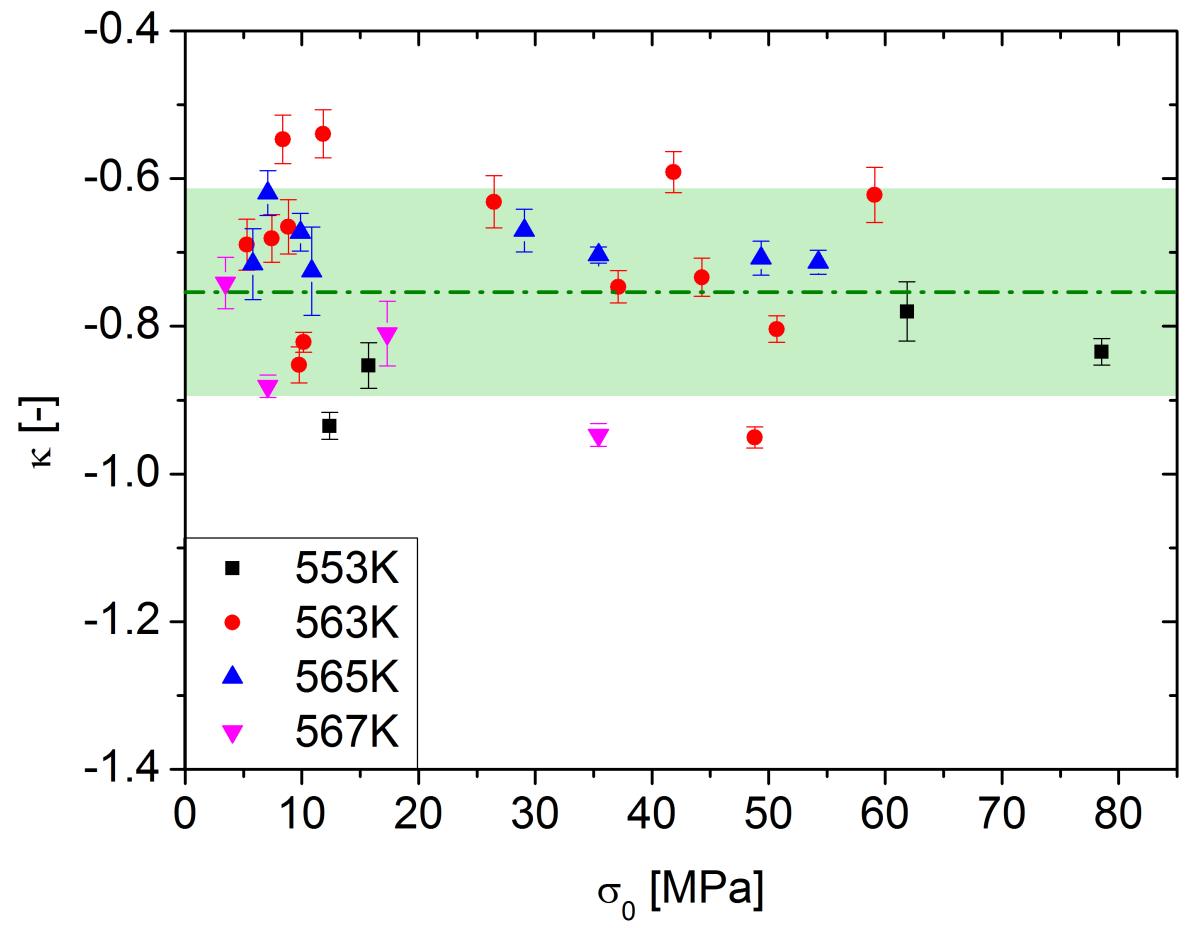

(b) For $\Delta \epsilon_{i t v l}<0$

Figure 5.31 Exponent $\kappa$ based on the linear regime of double-logarithmic distribution on interval strain $\left\langle\left(\Delta \epsilon_{i t v l}\right)_{i j}^{L}\right\rangle$ (cf. e.g. fig. 5.29 and fig. 5.30). (a) for positive and (b) for negative intervals. 


\section{Chapter 6}

\section{Discussion}

\subsection{Characterization of Sample Properties}

The amorphous state of the as-cast and the mechanically loaded samples is confirmed by XRD-scans. The broad halo observed in the spectra in fig. 4.1 arises from the long-range disorder, which is not influenced by LAOS-measurements as illustrated by the comparability in the behavior of samples $i$ to $v$ and samples vi to vii.

The quantification of the contributing elements of the cast metallic glass is confirmed by EDX-measurements on a broad range of samples and yields within the limits of the accuracy of the method the expected composition of $\mathrm{Pd}_{40} \mathrm{Ni}_{40} \mathrm{P}_{20}$.

The calorimetric analysis confirms these results, as DSC-scans show a distinct glass transition. The onset of the glass transition $T_{g}^{D S C}$, the onset of first crystallization $T_{x 1}$, and the width of the regime of the supercooled liquid $\Delta T$ are in perfect agreement to results by $\mathrm{Kahl}$ et al. [66]. The difference in the specific heat for the glass and the supercooled liquid $\Delta c_{p}^{s-1}$ is $81 \%$ of the value measured by Wilde et al. [151] and $10 \%$ higher than the value measured by $\mathrm{Hu}$ et al [56] and thus reflects the expected calorimetric behavior for metallic glass $\mathrm{Pd}_{40} \mathrm{Ni}_{40} \mathrm{P}_{20}$ samples with respect to literature data.

Temperature scans according to DMA-measurements yield a glass transition temperature $T_{g}^{P}$ at $598(2) \mathrm{K}$. It is determined by the position of the loss peak of the modulus, which coincides within the limits of accuracy with the value for $T_{g}^{P}$ measured by Schröter et al. [125] using torsional rheology. A comparison of the shear modulus measured at $320 \mathrm{~K}$ with values extracted at high frequencies by the rheological measurements show the expected agreement within the limits of data accuracy. Values reported on the basis of ultrasonic measurements [66. 79] yield a shear modulus which is higher by a factor of about 1.6 in comparison to the values extracted by mechanical excitation for both torsional and bending geometry.

The good agreement between the results of the experimental characterization of the metallic glass $\mathrm{Pd}_{40} \mathrm{Ni}_{40} \mathrm{P}_{20}$ samples used for LAOS-experiments in this thesis and comparable data reported in literature confirms the achievement of the required quality and properties of the samples.

\subsection{Qualitative Response Behavior based on Lissajous-Figures}

In order to give a qualitative evaluation of the characteristic mechanical behavior in dependence on the stress amplitude $\sigma_{0}$, the temperature $T$, and the evolution with time $t$, Lissajous-plots are described in section 5.1. They provide the relation of complex stress $\sigma^{*}$ and complex strain $\varepsilon^{*}$ for one period, thus the amplitude of the stress $\left|\sigma^{*}\right|$ is also referred to as the intra-periodic stress, which is equivalent to $\sigma_{c}$ as declared in section 3.7 . 
Fig. 5.1 depicts the influence of the stress amplitude $\sigma_{0}$ on the sample response at constant temperature.

For the smallest stress amplitude, the Lissajous-figure shows a linear form which is typically connected to a purely elastic response behavior within the limits of resolution.

With increasing stress amplitude a loss contribution evolves, which is clearly identified by the elliptical shape of the stress-strain-data. The area within the ellipsis is equal to the energy dissipated during the period, which increases with increasing stress and reflects the intensifying viscoelastic character of the material's response [92]. Losses can be attributed to the activation of relaxation processes, which are obviously induced by the applied stress as reported in literature [92, 157].

For even higher stress amplitudes the shape becomes nonlinear. At small intra-periodic stresses the slope of the stress-strain-loop changes towards lower values. In the region of the minima and maxima of the sine wave, the value of the intra-periodic stress is close to the stress amplitude of the experiment $\left(\sigma_{c} \approx \sigma_{0}\right)$. Here, the slope of the stress-strain-data is equal to the value observed under lowest excitations, and is thus regarded as independent of the stress amplitude.

Fig. 5.2 gives an example for the effect of the temperature $T$ on the shape of the Lissajousfigure. It demonstrates that the elliptic form and thus the loss in the material intensifies, corresponding to an increasing activation of relaxation phenomena with temperature. The nonlinearity becomes more prominent as the temperature increases, similar to the effect of increasing stress amplitude on the Lissajous-figures.

The evolution of the Lissajous figures with time under excitation at constant temperature and stress amplitude is represented by fig. 5.3. It becomes evident that the stress-strain-signal changes significantly with time, while the Lissajous-figures seem to become more nonlinear. The impression of a more intense nonlinearity for chronologically following Lissajous-figures is due to the evolving change of the slope in the region of small intra-periodic stresses, where $\sigma_{c}<<\sigma_{0}$. The slope at higher intra-periodic stresses remains constant.

\subsection{Nonlinear Response Behavior based on Fourier-Analysis}

As presented in section 5.3, quantitative results on the nonlinearity of the sample response and thus of the compliance $\chi$ are gained by the analysis of the Fourier-amplitudes and phase shifts at fundamental and higher harmonic frequencies.

In figure 5.5 an overview on the harmonic contributions in strain amplitude according to the Fourier-analysis is given. These results confirm the qualitative picture gained from the Lissajous-plots as nonlinear contributions of the Fourier-amplitudes are observed at sufficiently high stress amplitudes, while the signal remains linear for small stresses during initial low field.

During initial low field excitation, the higher harmonic contributions do not exceed the noise level, which is assumed as $3 \times 10^{-4} \%$ for the strain response. The fundamental frequency fully dominates the response behavior in a linear way. This is in accordance to results obtained by dielectric spectroscopy (DES)-measurements [147, 116, 118, 84].

In the case of emerging nonlinearity under high field excitation, the highest response amplitudes are observed at first and third harmonic frequency. Even order contributions do occur with small absolute values and are attributed to a misalignment of the neutral position (see appendix for further information).

Higher order contributions as the fifth harmonic exhibit amplitude values above the noise level for a number of measurements. It allows for a qualitative analysis which is presented in the appendix, as the data does not provide a sound range of stresses and temperatures. Still, 
as expected, the absolute value of the nonlinear coefficient of the fifth harmonic increases with increasing temperature [2].

The second low field excitation exhibits a highly time-dependent nonlinear contribution at the first harmonic frequency. Higher harmonic contributions do not occur, as these are still dependent on the stress amplitude $\sigma_{0}$. This is exemplified by $\left(\varepsilon / \sigma_{0}\right)_{3 \omega}$, which is equal to $\chi_{3} \sigma_{0}^{2}$ in eq. 3.14 and does not exceed the noise level for small stress amplitudes.

As depicted in fig. 5.6 and 5.11a, the nonlinear storage and loss response of the first harmonic frequency typically shows a KWW-type increase during high field and a decreasing KWW-behavior under second low field. KWW-fits in both regimes exhibit good agreement with the experimental data, if a KWW-exponent of 0.65 is applied in the fitting process. Three parameters are extracted from the KWW-fits, which are the instantaneous amplitude $A_{K W W}$, the continuous amplitude $B_{K W W}$, and the timescale of the response $\tau_{K W W}$ (cf. section 3.6.3).

The sum of instantaneous and continuous KWW-amplitude, $A_{K W W}+B_{K W W}$, yields the steady state value under a given stress excitation. In fig. 5.6 and 5.11 it is evident that this ideal plateau is not achieved by the experimental data. Instead of an ideal saturation to steady state, the amplitude continuously increases. This behavior reflects the flow-contribution of the material, based on its viscoelastic character, which is enhanced by the application of high stress at elevated temperature. The flow contribution mildly affects the extracted timescale $\tau_{\text {rec }}$ under high field, resulting in a tendency towards longer timescales.

The influence of the irreversible deformation also becomes evident by the steady state plateau at second low field, which is above the initial low field value for many measurements. This is reasonable, if plastic events are triggered during high field excitation. Plastic response behavior is connected to irreversible rearrangements within the sample (cf. section 2.5) which influence the sample geometry and thereby per definition the modulus. Even for very small changes in sample geometry a correction of the geometry factor is necessary to achieve the material's intrinsic modulus. Thus it has to be taken into account that the change in modulus is to a certain degree due to the change of the sample's geometry. It is important to ensure that the geometry-dependent change in modulus does not dominate the change induced by the LAOS experiment during high field excitation. Thus, only those measurements are included in the Fourier-analysis for which the difference between initial low field and second low field modulus is smaller than the sample-to-sample fluctuation of the low-field modulus, which is about $10 \%$ as measured by temperature-scans (cf. fig. 5.4.).

The difference in the behavior observed for high field and second low field response regarding the achievement of a steady state gives information on the degree of activation of plastic events. The occurrence of irreversible, i.e. plastic events under high-field excitation is evident as described above. This is not the case during second low field, as the plateau is for most cases indeed reflected by a constant value of the nonlinear response contribution. The compared stress amplitudes belong to the same measurement and the applied temperature is the same. Thus, it is the higher stress amplitude that induces plastic deformation. The response under low stress amplitude both for initial and second low field seems thus to be connected to elastic response behavior, while for higher stress amplitude it shows a behavior that is characteristic for an anelastic sample response.

In case of measurements at low temperature (fig. 5.11b), the loss contribution shows a decreasing evolution of the KWW-amplitude. It reflects the persistent aging of the sample during both initial low field and high field. That the origin of the decreasing response is due to a change of sample geometry is excluded, as the corresponding storage signal does not show the decrease in the response signal. The annealing prior to the mechanical experiment and also the duration of the initial low field part itself is not sufficiently long to bring the sample to steady state. This behavior stresses the fact that the sample response observed 
for measurements clearly below the glass transition temperature must be regarded as not fully reflecting quasi-equilibrium behavior, as the sample is still within the aging process.

The change in compliance within the stress-strain-loop is reflected by the interplay of first and third order contributions in the strain response from a geometrical point of view, as depicted in fig. 6.1. Here, the linear and nonlinear contributions occurring under high field excitation are scaled to another as they are measured during a stress amplitude of $54 \mathrm{MPa}$ at a temperature of $565 \mathrm{~K}$ (cf. fig.5.5). The blue line corresponds to the sine that would be expected if the sample were responding perfectly linear. The dashed red line corresponds to the contribution at fundamental frequency, thus its amplitude is higher than in the linear case due to the additional nonlinear contribution $\Delta \ln \varepsilon_{1 \omega}$. The red dotted line represents the response at third harmonic frequency $\varepsilon_{3 \omega}$. The overall strain response, which is the sum of the dotted and the dashed lines, exhibits a distorted sine as depicted by the full red line. In comparison to a pure sine wave it displays much steeper flanks in the vicinity of the inflection points, but also a much shallower progress around minima and maxima. Thus technically, the first harmonic nonlinear contribution gives rise to the super-linear strain response, while the third harmonic contribution reflects the reduction of the nonlinear contribution to the strain response at high intra-periodic stress, i.e. at $\pi / 2$ and $3 \pi / 2$ in fig. 6.1

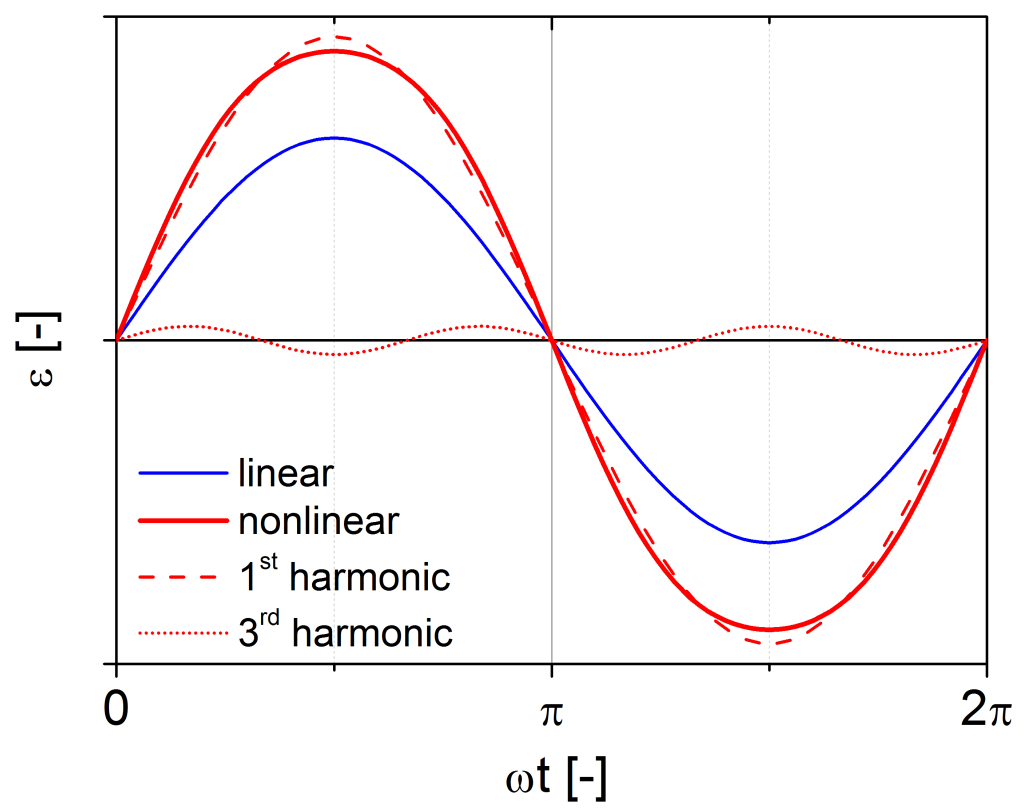

Figure 6.1 Scheme of the contributions resulting in the overall nonlinear strain response (solid red line) for one period. The solid blue line depicts the linear contribution of strain response, the red dashed line is due to both linear and nonlinear contribution at the first harmonic frequency, the red dotted line is based on the contribution at the third harmonic. Amplitudes and phase-shifts reflect the relations of the measurement shown in fig. 5.5

At the fundamental frequency, the nonlinear response amplitude increases with increasing temperature and increasing stress amplitude (cf. figures 5.7, 5.8, 5.12, and 5.13). This is characterized by the first harmonic nonlinear coefficients, which are plotted in fig. 5.9 and 5.14. They are deduced from the proportionality between nonlinear response amplitude and squared stress amplitude (cf. eq. 3.22 and 3.23). In semi-logarithmic scaling the nonlinear coefficients show a linear increase with increasing temperature for storage and loss contributions at the fundamental frequency for both high field and second low field. The values differ within the limits of the errors extracted by linear fits and express an increase of the nonlinear contributions by one order of magnitude within a temperature window of 11(2) K. The values for the ordinates are small for all coefficients and are thus regarded as zero. This part 
of the analysis reflects that the underlying process leading to nonlinear changes is identical for storage and loss compliance. Moreover, this process occurs both under load during high field and under second low field excitation when the system recovers.

While the nonlinear contribution at the first harmonic frequency leads to an additional accumulation of strain and thus an increase in compliance, the third harmonic nonlinear coefficient yields a decreasing effect. The absolute value of the third harmonic nonlinear coefficient shows an increase with temperature as well, even though it is not of the same nature as for the fundamental frequency (cf. fig. 6.2). In case of the relative change in third harmonic strain of Polymethylmethacrylate (PMMA) [44] a non-zero offset $y_{0}$ is observed as well, which is in a comparable order of magnitude compared to the absolute values involved in the fit on the metallic glass data.

Thus, the temperature dependence as well as the reducing effect on the compliance of the third harmonic contribution is of comparable nature in case of LAOS-experiments. In dielectric spectroscopy the third harmonic coefficient has an opposed effect on the susceptibility, as it enhances the response signal in the same way as the first harmonic contribution [84]. For this reason, the origin of the third harmonic might also be of a different kind for DES and DMA-experiments.

For DES-measurements, a connection of the third harmonic nonlinear coefficient to the intensity of particle-correlation is reported [16, 23, 84]. It results in the number of correlated particles $N_{\text {corr }}$ which is extracted from the third harmonic nonlinear coefficient $\chi_{3}^{(3)}$, which disposes a hump in dependence on measurement frequency. The knowledge necessary to obtain the number of correlated particles is the amplitude of the nonlinear coefficient at the maximum of the hump, or at a constant spectral distance to it. The extraction of the number of correlated particles and a comparison to literature data on correlation length scales measured by other methods [104, 127, 120] would give an estimate on the applicability of the model by Biroli and Bouchaud [16] on LAOS-data. The LAOS-experiments are performed at fixed frequency, thus no further spectral information is available than the knowledge of $\chi_{3}^{(3)}$ at a frequency of $1 \mathrm{~Hz}$. Thus, a quantitative value for $N_{c o r r}$ is not extracted by the LAOS-measurements. However, comparison to the spectral evolution of the third harmonic nonlinear coefficient for glycerol from DES-measurements yields the same trend [8], i.e. an increase in $\chi_{3}^{(3)}$ with increasing temperature. The occurring resonance of the DMA-machine at frequencies slightly higher than $1 \mathrm{~Hz}$ restricts the frequency domain significantly. To do so a dynamical mechanical analyzer is required, which generates reliable stress and strain data over a frequency regime of 2 to 3 order of magnitudes, even for materials with a relatively high modulus in the GPa regime as the case for metallic glasses. The DMA 8000 is designed for the analysis of polymers, ideally covering materials of a modulus between $106 \mathrm{~Pa}$ and $1016 \mathrm{~Pa}$ [105].

A comparison of nonlinear coefficients extracted for first and third harmonic frequency is shown in fig. 6.2. Even though the effect of first and third harmonic coefficient is of opposite effect, the nonlinear coefficients exhibit absolute values, which are roughly in a comparable order of magnitude. This observation is similar to the relation between nonlinear coefficients extracted from DES-experiments [8, 148], e.g. for glycerol. The increase of the nonlinear coefficients at fundamental frequency reflects the dominating dependence of the compliance on the temperature $T$ and the stress amplitude $\sigma_{0}$.

The similarity between the effect induced by an increase in stress amplitude and an increase in temperature is in some cases discussed in terms of a change in fictive temperature [38]. The change $\Delta T_{f}$ for the stressed case is induced by the external field as assumed by the Box-model [117] and applied for results from dielectric spectroscopy, for which the thermal energy is much larger than the energies induced by the electric field [117, 148, 113, 57]. If an amorphous system is excited by an external field at a given frequency $f$, certain con- 


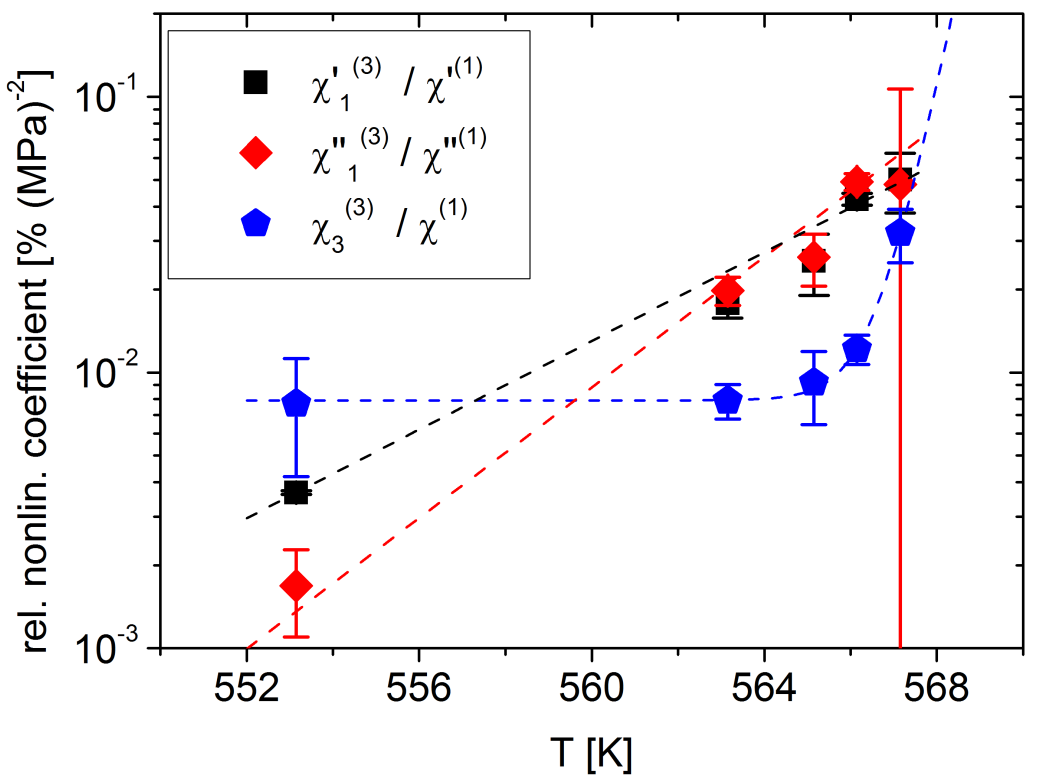

Figure 6.2 Relative nonlinear coefficients for first harmonic storage and loss, and third harmonic complex contribution plotted against temperature $T$.

figurational modes couple to the exciting signal and gain energy. If these configurational modes couple only weakly to modes in its vicinity (spectrally or locally), and show a rather weak link to vibrational modes, energy will be accumulated by this mode. It takes a longer time to relax the energy to the phonon bath or to neighboring modes than to absorb energy from the external field. The accumulated energy affects the excited mode in the sense of an extra energy, described by an increase in fictive temperature. This increase leads to a change in the characteristic sample response, which changes towards the response which is typically expected at the temperature $T+\Delta T_{f}$. The time-dependent behavior is reflected by the timescale $\tau_{\text {rec }}$ that is needed to achieve a steady state between energy absorption and recovery. However, this interpretation should be regarded with care if applied on metallic glasses, as model calculations for $\mathrm{Pd}_{40} \mathrm{Ni}_{40} \mathrm{P}_{20}$ do not yield satisfying agreement between experimental and modeled data [114]. As the energies transferred in the mechanically excited system are large compared to $k_{B} T$, the assumptions made by the box-model might not hold.

In the $\mathbf{P E L}$ the difference in the effect of temperature and stress becomes evident. An increase in temperature leads to more intense fluctuations as indicated in fig. 6.3 from [101] by the green arrow. The excitation by a mechanical field yields a directional tilt of the PEL as depicted by the red line and arrows. It is very different from the effect of temperature, which acts on a random basis, i.e. non-directional. This becomes even more intense, if the mechanically induced energy becomes large compared to the thermal energy of the system. In the MD-simulation performed by Yu et al. [157] the effects of temperature and mechanical excitation amplitude are investigated, which are also reported by [126, 50].

This description can be used for the interpretation of the response behavior. Due to the applied field, barriers in the PEL can be overcome, which are too high to be surmounted by thermally induced hopping in the unstrained or only mildly strained case. If the stress amplitude is sufficiently high, it is connected to such an intense tilt of the PEL that the system is not only able to change between inherent states but also passes into other metabasins [157, [126, 50]. These transitions are identified with an irreversible change within the microstructure, which is assumed to occur due to the activation of STZ $\$$ (cf. section 2.5. fig. 2.13). The quadrupolar elastic matrices surrounding the plastic core of STZ $\$$ can trigger further $[\mathrm{STZ} \beta$ resulting in a cooperative rearrangement of irreversible nature. This process is connected to a breakdown of elasticity as described by the EIP-motif [77]. 


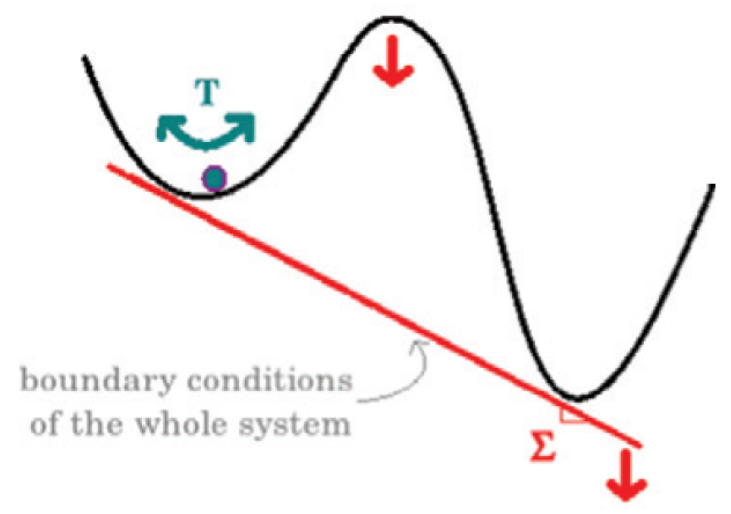

Figure 6.3 Scheme of the different effects of temperature $T$ and mechanical excitation (here referred to as $\Sigma$ ) from [101].

The intense tilting of the $\mathrm{PEL}$ also shows an effect on the modulus of the material. By the tilting of the potential the basins are changed to a less intense curvature, resulting in a lower modulus [161, 52, 63]. The effect of the applied stress on the modulus and thus on the compliance is quantified by the evolving nonlinearity at fundamental frequency.

The $\mathrm{PEL}$ provides a possible explanation for the different effects of first and third harmonic contribution on the compliance. It is connected to the influence of the sinusoidal form of the excitation signal and the connected strain rate. A reduction in nonlinear strain contribution corresponds to a lower compliance, i.e. a higher modulus. The modulus is connected to the curvature of the minimum in the $\mathrm{PEL}$ that the system inherits. As the strain rate ceases around $\sigma_{c} \approx \sigma_{0}$, also the tilt of the $\mathrm{PEL}$ comes to rest and the system has time to relax within the metabasin the system is currently trapped in. The curvature at deeper regions of a metabasin is assumed to be higher, thus the modulus of these states is higher, too.

Both temperature and stress are capable of driving the system towards its less rigid state [157, 126, 50]. If the temperature is increased sufficiently, the accompanied transition is called the glass transition [38]. If the excitation to this transition is of mechanical nature, it is referred to as the mechanical yielding transition [108, 52, 61]. Stress and temperature accompany each other, thus at elevated temperatures less stress is needed to drive the system to the yielding transition than the case at low temperature [50, 157].

In the experimental realization of the LAOS approach, the material is mechanically excited at temperatures slightly below $T_{g}$ and thereby driven towards yielding. The maximum strain of the experiments are within $0.02 \%$ and $0.13 \%$. Thus even the strain response to the highest stress amplitudes applied is by an order of magnitude lower than the universal yield strain according to the $\mathrm{CSM}$, which is $\gamma_{c}^{C S M}=2 \%$ [64]. On the basis of static stressstrain-curves (cf. section 4.4) which are extracted at temperatures typically applied in the LAOS-measurements, a similar conclusion can be drawn, as depicted in fig. 4.4. Still, the viscoelastic behavior, which is connected to irreversible rearrangements, is one of the indicators for the approach of yielding. A comparison of the nonlinear response at high and low field excitation clearly shows a difference in the response behavior. While the response is highly influenced by irreversible response behavior under high field excitation, the low field response shows characteristics of elastic behavior. This approach is directly depicted in fig. 5.4 which shows a comparison of the storage compliance deduced from LAOS-measurements and the storage compliance determined by low-field temperature scans. The low field compliance of the LAOS-experiments is in comparable order to the results from temperature scans. The high field compliance in contrast shows clearly rather enhanced values, that are rather comparable to compliance data at temperatures above the onset of the glass transition $T_{g}^{\text {on }}$. A connection of the increase in fictive temperature by $\Delta T_{f}$ due to a mechanical excitation lacks on the quantitative scale as described before. Still, a connection between the compliance un- 
der high-field excitation and the compliance at elevated temperature is clearly demonstrated by the data. Thus, the response to high stress amplitudes is connected to a shift towards the anelastic response regime on a qualitative basis.

Another indication for the connection of the high-field response to irreversible relaxation phenomena and thus the approach of the yielding transition is found in the recovery timescale $\tau_{r e c}$, which reflects the evolution of nonlinearity. The timescale obtained by KWW-analysis describes how quick the steady state is approached during high field excitation and second low field excitation [58, 118]. It is a measure for the timescale describing the evolution of the nonlinearity. In DES experiments this timescale is termed the timescale of structural recovery $\tau_{r e c}$ [118]. The timescales extracted from the storage compliance show very similar values for high field and second low field as expected (cf. fig. 5.10).

The analysis performed on the loss compliance shows larger KWW-timescales under second low field than under high field excitation (fig. 5.15). For both storage and loss contribution matching timescales for high field and second low field are expected [155, 10]. The reason for the deviation reflects the uncertainties due to the measurement and analysis technique. To facilitate the comparison of the time-dependent behavior at both first and third harmonic frequency, the timescales extracted from data measured during high field excitation are plotted against temperature in fig. 6.4. The timescale extracted from storage compliance at the fundamental frequency $\tau_{\chi^{\prime}, 1 \omega}^{h f}$ (solid squares) is rather constant with temperature. Both the timescale of loss compliance $\tau_{\chi^{\prime \prime}, 1 \omega}^{h f}$ (open squares) and of the compliance at third harmonic frequency $\tau_{\chi, 3 \omega}^{h f}$ (solid stars) show an increase with decreasing temperature. The accuracy of the measurement does not allow for a reliable differentiation between the trends of the three different recovery timescales. Thus the overall behavior of the recovery timescale is assumed to be reflected by the blue dashed line in fig. 6.4, which is a guide to the eye.

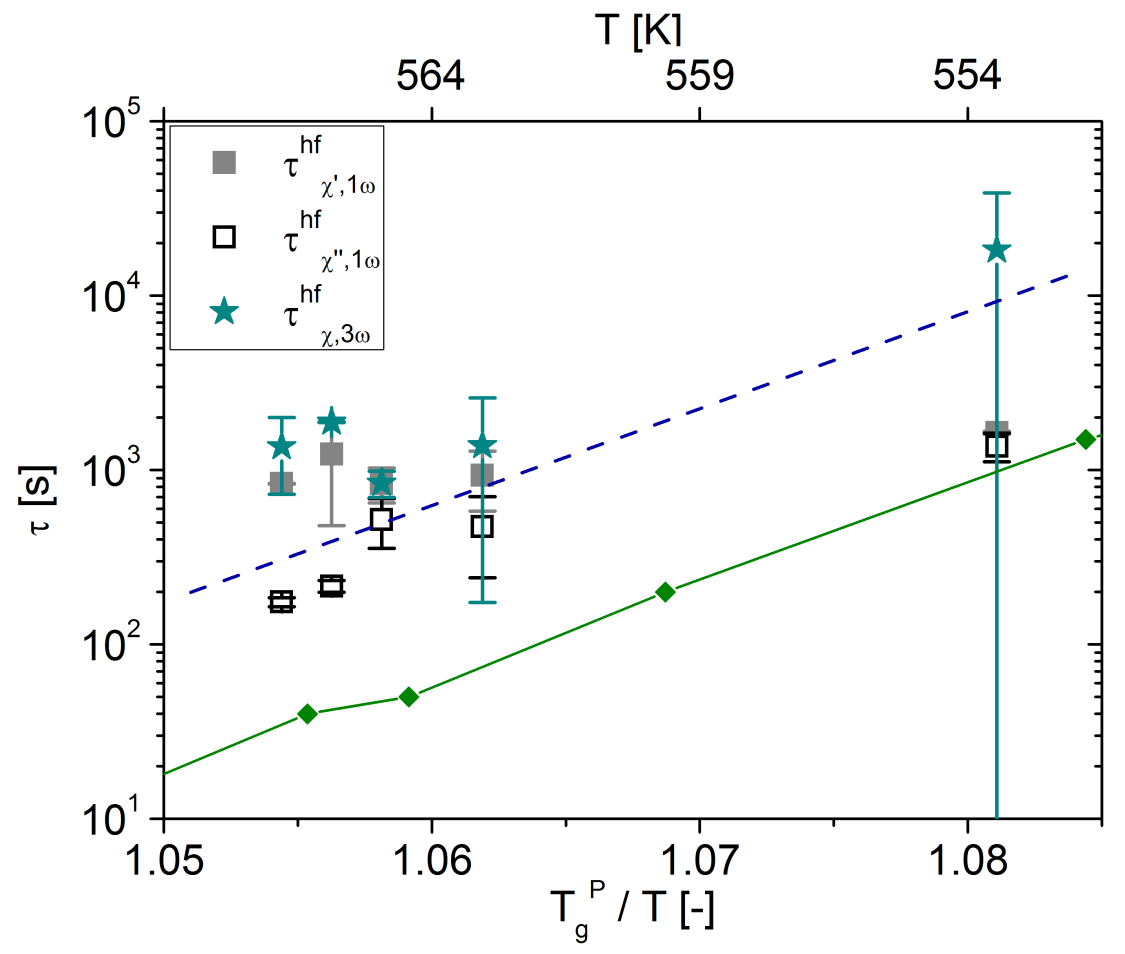

Figure 6.4 Temperature-dependent timescales for first harmonic storage and loss compliance, $\tau_{\chi^{\prime}, 1 \omega}^{h f}$ and $\tau_{\chi^{\prime \prime}, 1 \omega}^{h f}$, and third harmonic complex compliance $\tau_{\chi, 3 \omega}^{h f}$ under high field excitation as in figures 5.10 5.15 and 5.19 depicted by symbols. Literature data on the structural relaxation timescale $\tau_{\alpha}$ [151] correspond to green symbols connected by lines. The blue dashed line serves as a guide to the eye for the general trend of the measured recovery timescales with temperature. 
The overall trend of the extracted timescales for first harmonic loss and third harmonic complex compliance show an increase in $\tau$ with decreasing temperature. A comparison to literature data on the timescale of structural relaxation $\tau_{\alpha}$ [151] shows, that the change with temperature is comparable, while the data shows on average an offset of one order of magnitude towards higher timescales for all investigated temperatures. Even if the trend of the recovery timescale does not exhibit a perfect match with the structural relaxation timescale, the data shows that the evolution of the nonlinearity is closely connected to $\tau_{\alpha}$ and thus to the occurrence of irreversible rearrangements. It does not reflect the timescale of the applied frequency $\tau_{f} \approx(2 \pi f)^{-1}$ of the measurement, which is about $160 \mathrm{~ms}$ [58, 118]. The fact that the extracted timescale is connected to the timescale of structural relaxation of the material rather than to the timescale of the excitation frequency stands in contradiction to the assumption of the box-model (cf. section 2.7.1). As the model assumes a negligible exchange between different relaxation modes, the only timescale that should play a role in the recovery of energy is the intrinsic timescale of the excited mode with $\tau_{i} \approx(2 \pi f)^{-1}$ under the assumption that the calorimetric coupling is corresponding to $\tau_{i}$. The overall relaxation time characterizes the recovery of the accumulated energy and reflects the average relaxation timescale of an ensemble of configurational relaxations, i.e. the matrix response or at least the average response of a large number of individual relaxations. This gives clear evidence, that either spectrally or spatially long-range interactions between relaxations play a dominant role. As the metallic glass $\mathrm{Pd}_{40} \mathrm{Ni}_{40} \mathrm{P}_{20}$ is regarded as a structural glass former, which is directly exposed to a mechanical field in the LAOS-experiment, the assumption of the excitation of structural rearrangements is the logical consequence. The long-range interaction is thus to be assumed on the spatial scale, e.g. via the long-range Eshelby stress field of occurring STZ $\$$ [42, 41]. This is different for experiments by means of DES, as the electric field couples to the dipolar moment of molecules, which oscillates in a damped mode with the exciting field. To which extent the dipole couples to the backbone of the molecule is not always clear even though a close coupling is observed for a number of materials [119]. Neither is known if it is possible to trigger rearrangements in terms of avalanche dynamics in DES texperiments.

\subsection{Response Behavior based on the Incremental-Analysis}

The definition of intervals as described in section 3 allows for a thorough analysis of the dependence of the response on the applied parameters of the measurement. The incremental analysis is an analytical method individually developed for the evaluation of the LAOS. experiments. It is based on the concept of avalanche dynamics theory according to the EIP-motif [77] and similar findings [88, 32, 142]. If avalanche dynamics dominate the response behavior, the incremental analysis will result in power-law behavior for the distributions of the interval response. If for example noise were the dominating factor in the response data, the extracted distributions would exhibit a Gaussian form, and thus the absence of power-law behavior is the expected result.

According to [140] the size of avalanches increases with the applied stress. Thus, the closer the system is pushed towards yielding, the more intense the plastic contribution to the sample response becomes. This finally leads to system spanning events in form of permanent shear bands, which occur in the plastic regime of the stress-strain-curve at stresses clearly above the yielding transition [49]. The events dominating the response of the LAOS-experiments are assumed to be due to avalanches as observed strain values are far below the critical yield strain [64]. If these events would occur temporally and spatially separated, the signature is assumed to be comparable to the EIP-motif [77, 32]. It consists of a segment of linear response exhibiting the modulus or compliance observed at low temperatures and low fields, and a segment due to the plastic event. In a strain-controlled experiment, which is the basis of the depicted EIP-motif in fig. 2.31, the plastic event is measurable as a drop in stress. The drop intensity and duration are highly dependent on the strain rate and show infinitesimal 
small durations in the athermal limit [142]. In case of a stress-controlled tensile experiment the instability is expected to occur as a jump in strain. In case of a single cantilever bending experiment, simulation data depicting the EIP-motif are not at hand, thus it is speculative if the instability is definitively of increasing strain, or if a drop in strain is similarly possible, as the bending geometry results in a dilated and a compressed zone simultaneously [154]. The sample response regarding the EIP-motif might not be as intuitive as the case for a tensile experiment. Moreover, it assumes that only one avalanche occurs at a time. Due to the elevated temperature of the performed LAOS-measurements and the sample dimensions, which include a huge number of particles, such a temporal separation of avalanches is not a realistic assumption.

The incremental analysis of the data results in positive and negative intervals which are analyzed regarding averaged values of interval strain and interval duration as shown in section 5.4.2. On the basis of interval strain data, distributions are discussed regarding similarities to critical behavior [106, 150, 131], which is typical for avalanche-dominated material response. The dependence on temperature $T$, stress amplitude $\sigma_{0}$, and intra-periodic stress $\sigma_{c}$ is investigated, as well as the influence of the strain rate $\dot{\varepsilon}$.

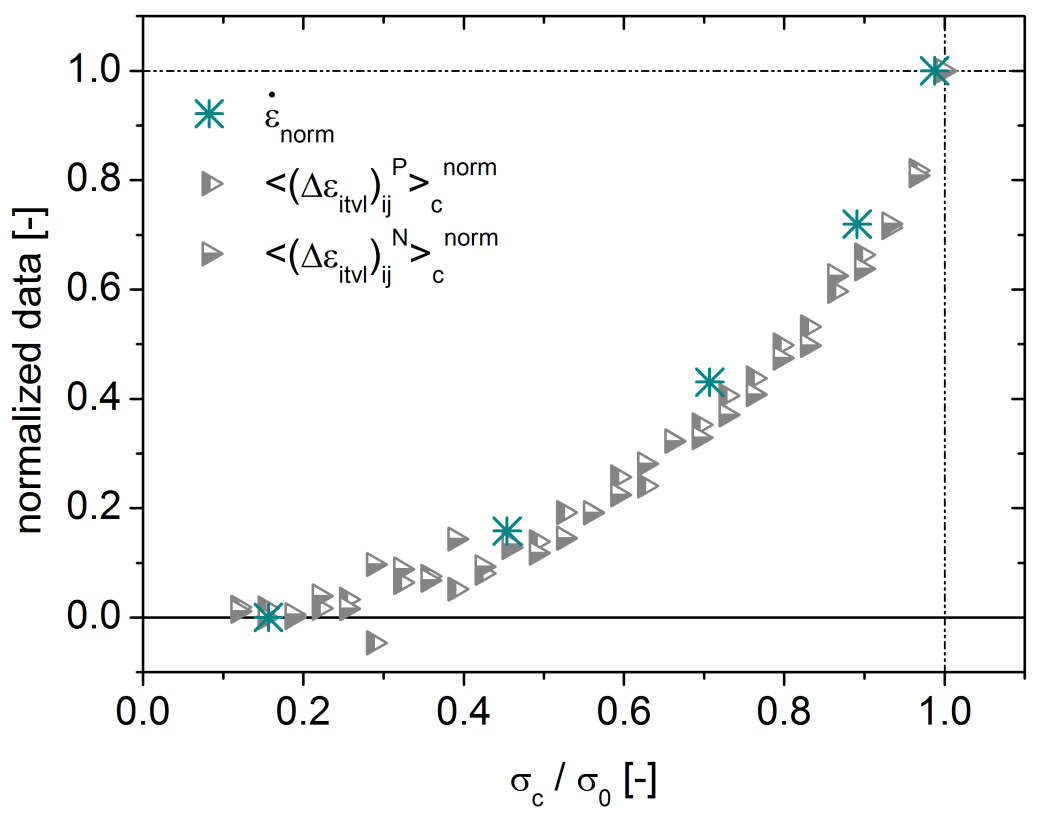

Figure 6.5 Normalized strain rate $\dot{\varepsilon}_{n o r m}$ and normalized averaged interval strain $\left\langle\left(\Delta \epsilon_{i t v l}\right)_{i j}^{L}\right\rangle_{c}^{\text {norm }}$ plotted against the normalized intra-periodic stress $\sigma_{c}$ for a stress amplitude of $\sigma_{0}=60 \mathrm{MPa}$ at constant temperature $T=563 \mathrm{~K}$.

The averaged interval strain $\left\langle\left(\Delta \epsilon_{i t v l}\right)_{i j}^{L}>\right.$ strongly varies with the applied stress. On the scale of the intra-periodic stress $\sigma_{c}$ the averaged interval strain decreases, while it increases with increasing stress amplitude $\sigma_{0}$. Normalization of the averaged strain data performed according to eq. 5.1 and a rescaling of the $x$-axis by division by $\sigma_{0}$ leads to the collapse of the data onto a mastercurve (cf. fig. 5.23). If the normalized strain rate as described in section 5.4 .1 and plotted in fig. $5.20 \mathrm{~b}$ is compared with the mastercurve of the averaged interval strain, a strong similarity in the evolution on the intra-periodic scale is observed as depicted in fig. 6.5. Triangles correspond to the normalized averaged interval strain for positive and negative intervals, while the asterisk symbols depict the evolution of normalized strain rate. All three normalized quantities show a matching evolution with $\sigma_{c} / \sigma_{0}$. Furthermore, plotting the normalization factors of the averaged interval strain for positive and negative intervals against the normalization factor of the strain rate, a linear relation is obtained as shown in fig. 6.6. Thereby, both the evolution with intra-periodic stress $\sigma_{c}$ and the increase with increasing stress amplitude $\sigma_{0}$ can be attributed to a dominating dependence on the strain rate $\dot{\varepsilon}$. This observation is in accordance to data reported in literature [54]. It results in an 
increase of the averaged interval strains with growing strain rate. The amount of change with increasing strain rate is of the same intensity for positive and negative intervals. Thus, with increasing strain rate the absolute values of averaged interval strain for positive intervals increase, while the absolute values for negative intervals decrease.

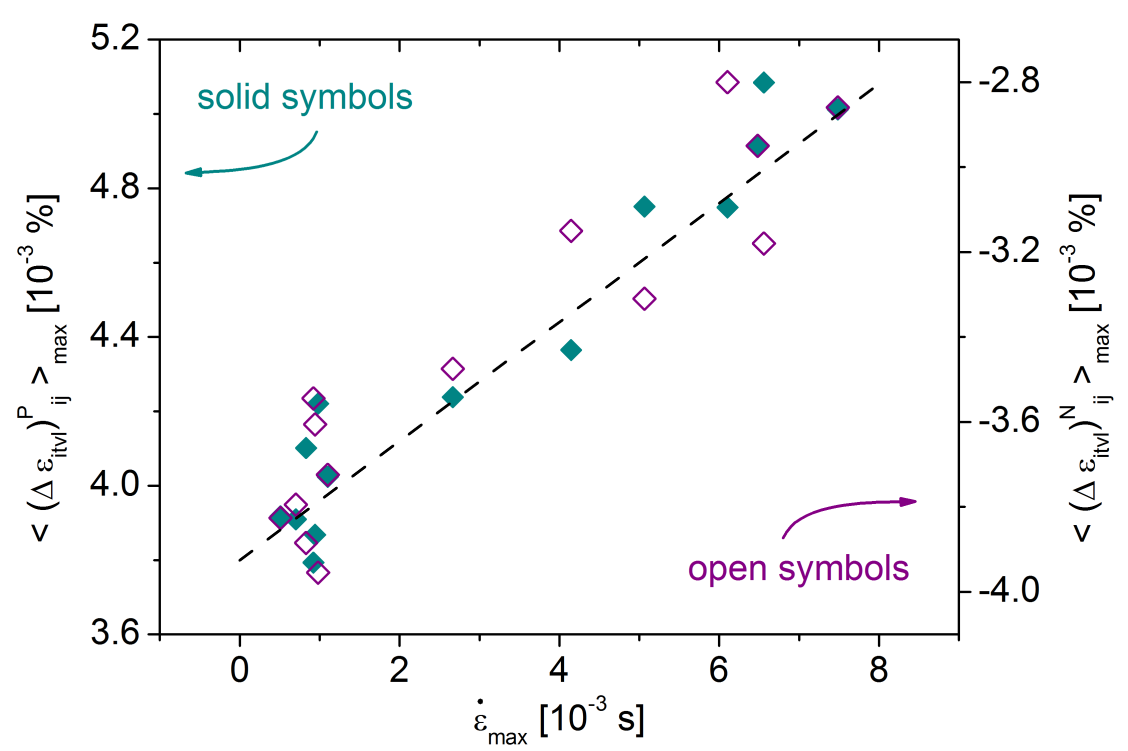

Figure 6.6 Normalization factor of the averaged interval strain plotted against the normalization factor of the strain rate for a stress amplitude of $\sigma_{0}=60 \mathrm{MPa}$ at constant temperature $T=563 \mathrm{~K}$.

The normalized average interval duration $\Delta t_{i t v /}$ shows a comparable evolution on the intraperiodic scale as the case for averaged interval strain values. This behavior is depicted in fig. 6.7a, for both positive and negative intervals. The dependence on the strain rate is dominant for this quantity as well, both for the evolution within a period and with changing stress amplitude. In case of interval duration, the averaged values decrease with increasing strain rate for both positive and negative intervals. This relation is plotted in fig. 6.7b which shows the normalizing factors for averaged interval durations against the strain rate.

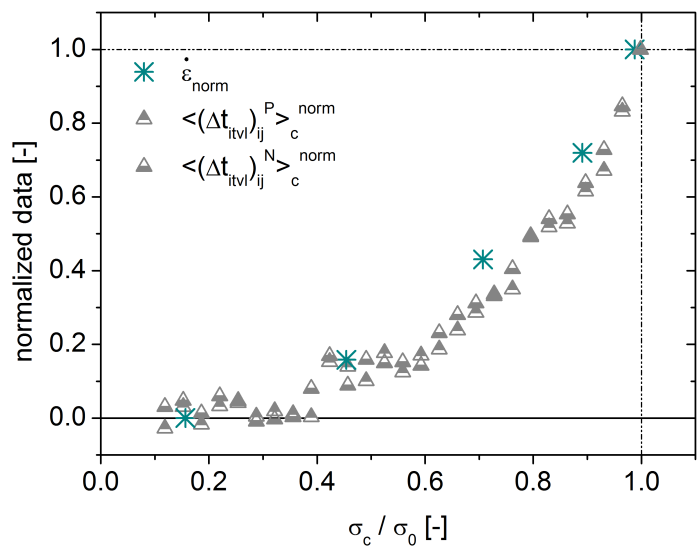

(a)

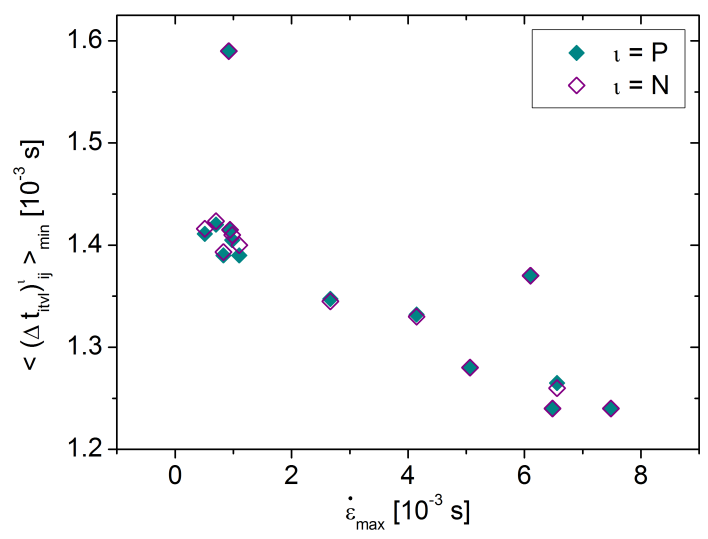

(b)

Figure 6.7 (a) normalized strain rate $\dot{\varepsilon}_{\text {norm }}$ and normalized averaged interval duration < $\left(\Delta t_{i t v l}\right)_{i j}^{\iota}>_{c}^{\text {norm }}$ plotted against normalized intra-periodic stress $\sigma_{c}$. (b) normalization factor of the averaged interval duration plotted against the normalization factor of the strain rate. Both plots are shown for a stress amplitude of $\sigma_{0}=60 \mathrm{MPa}$ at constant temperature $T=563 \mathrm{~K}$. 
The distributions of strain intervals occurring at $\sigma_{c} \leq 2 / 3 \sigma_{0}$ show qualitatively the same form for both positive and negative intervals (cf fig. 5.28). Three characteristics are observed for all distributions based on interval strain data. First, the power-law behavior that is prominent for low interval strains. Second, a hump occurring in the second half of the distributions. Third, the cut off which concludes the distributions.

The cut-off terminates the distributions at an absolute strain value of $17.8(8) \times 10^{-3} \%$, independent of the stress amplitude for both positive and negative intervals. In the plastic regime, the value of the cut-off is identified to be of the size of a system-spanning event [74]. In the elastic and anelastic response regime system-spanning events are not expected to occur, thus it is reasonable that the cut-off occurs at strain values smaller than the system's smallest dimension, which corresponds to about $10^{-2} \%$ strain.

The hump is a temperature- and stress-dependent feature of the distribution. Its maximum at low stress amplitudes coincides with the temperature-specific average interval strain (cf. fig. 5.28). The hump is reported in literature as well, connected to strain rates sufficiently high to overdrive weaker, i.e. smaller events, resulting in a triggered occurrence of larger events [150, 33, 135]. If this explanation is applicable to the LAOS-data has to be tested, for example by measurements over a broad range of strain rates. With increasing stress amplitude, i.e. increasing strain rate (see fig. 5.21a) the hump exhibits a certain shift. It is observed as a shifting towards higher strain values for positive intervals as depicted in fig. 6.8 , while it shifts towards lower strain values for negative intervals. This reflects qualitatively the behavior of the averaged interval strain, which is strongly influenced by the strain rate as well.

For the smallest observed values of interval strain between $10^{-4} \%$ and $10^{-3} \%$ a linear dependence between logarithmic probability and logarithmic interval strain is observed. This range corresponds to displacements between $40 \mathrm{~nm}$ and $400 \mathrm{~nm}$. According to the manufacturer's handbook [105] the spatial resolution of the dynamical mechanical analyzer is of $1 \mathrm{~nm}$ and thus well below the acquired interval strain data. The power-law regime exhibits an exponent of about -0.8 for both positive and negative intervals, which is independent of temperature, applied stress, and the type of intervals. Many simulations focusing on the extraction of power-law exponents in amorphous systems reflect strain-driven systems in the plastic regime [77, 32, 88]. The analysis of a stress-driven system under rheological or sinusoidal excitation within the elastic regime is not reported as far as the literature research reveals that was conducted in connection to this thesis. Thus, a literature value based on simulations is not at hand for comparison, even though a variety of different power-laws for stress drop statistics, energy distributions, and other parameters are published [88, 81, 142, 134, 74, 54]. Results extracted from molecular dynamics simulation [77, 81] indicate, that the exponents observed for elastic and plastic response behavior are comparable or even identical. This observation is also reported in case of Barkhausen noise experiments [140]. The findings regarding the exponent extracted from the interval strain distributions are in accordance with the exponent extracted by mechanical analysis on metallic glasses by Krisponeit et al. [74] from waiting time distributions. An analysis of the exponent's dependence on the strain rate confirms this comparability, as the strain rates connected to an exponent of -0.8 are low and thus suitable to the rates reported in this work [54].

As observed for the measurement at $553 \mathrm{~K}$ at a stress amplitude of about $60 \mathrm{MPa}$, in the distribution of negative intervals (cf. fig. 5.30b) the much less pronounced hump yields a distribution that exhibits power-law behavior over the complete range of observed interval strain, while the distribution of positive intervals is of characteristic shape (cf. fig 5.30a). As this temperature is connected to persistent aging as observed in the nonlinear contribution at first harmonic frequency of the loss compliance (cf. fig. 5.11b), it is possible that the more pronounced power-law behavior is in connection to the aging procedure. However, the low field data and data of other measurements reject a simple connection to aging, as these do not show the absence of the hump in the distribution. 


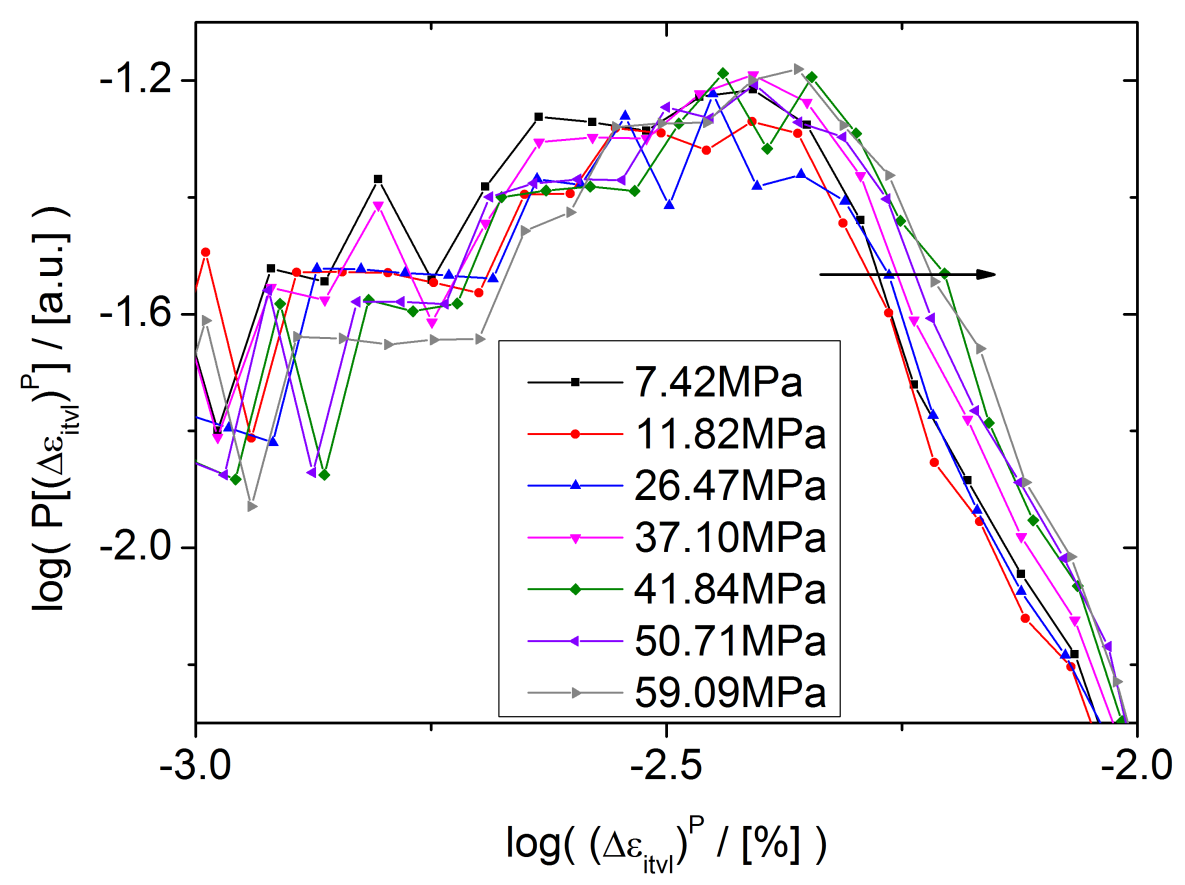

Figure 6.8 Zoom of the hump of the interval strain distributions for positive intervals as shown in fig. 5.28 for various stress amplitude $\sigma_{0}$ at constant temperature $T=563 \mathrm{~K}$. The arrow depicts schematically the observed shift of the hump with increasing stress amplitude $\sigma_{0}$, i.e. increasing strain rate $\dot{\varepsilon}$.

The position of the hump is temperature and stress dependent, but it is not suppressed by these parameters in the investigated parameter regimes. Thus the origin for the hump is not identified, even though it is obvious that some parameter, that is triggered in the LAOS. experiment or the preparation procedure, must be highly connected to the suppression of the hump in the interval strain distribution.

Two possible routes of interpretation of the interval data are described and discussed in the following paragraphs. As only one of these routes is logically favorable, arguments are given that reflect the conclusion why other options of data interpretation are discarded.

\section{Individual Events}

A possible interpretation of the data is the direct comparison of intervals to the EIP-motif. Positive intervals correspond to the linear, i.e. elastic part of the motif, while negative intervals are due to plastic events in the material [32. Then every maximum in fig. 3.6 corresponds to an instability point as depicted in the EIP-motif, and the minima mark the end of a plastic event, after which the elastic response dominates again [77].

Several points mark this description as a misleading interpretation of the data. First of all, the EIP-motif as depicted in fig. 2.31 is extracted under the condition of strain control. Thus, the stress is the only free parameter, which naturally shows a strong decrease if the sample undergoes plastic deformation and thereby releases a certain amount of energy. The LAOS experiment on the contrary is a stress-controlled experiment. The strain is the free variable in the system which is expected to undergo a certain change in case of a plastic response event. Whether this change is positive or negative is not necessarily self-evident due to the simultaneous existence of a dilated and a compressed zone in a sample under single cantilever bending conditions.

If one type of intervals were assumed as linear and the other as corresponding in ways of a plastic response, the one representing the elastic behavior is expected to have a positive value. Moreover, it should exhibit a constant interval strain at least on a small intra-periodic stress segment, or at least a more narrow distribution of interval strain. This behavior is not 
observed in case of the interval data, as it shows very similar behavior for both positive and negative intervals in case of averaged interval strain and distributions of interval strain. Both show a very similar distribution including the power-law regime.

The interval slope itself should show a distinct value throughout the whole stress regime for one type of intervals as it should reflect the inverse elastic modulus of the material in terms of a constant value in the order of the low field compliance (cf. fig. 2.30). However, both types of intervals have a similar type of compliance behavior as shown in the appendix.

For these reasons the direct interpretation of the data in terms of a pure EIP-motif is not applicable. The deviation between the response behavior and the characteristic EIP-motif is reasonable, as the LAOS-experiments are performed at elevated temperatures close to the glass transition. Irreversible relaxations play a dominant role during high field excitation, which are induced both due to the elevated temperature and stress. Due to the sample size, the response behavior is reasonably a superposition of both elastic and plastic response behavior. This suggests a more realistic interpretation of the data in terms of temporally and spatially overlapping rearranging events.

\section{Overlapping Events}

This route of interpretation assumes a temporal and spatial overlap of the occurring plastic events. This overlap leads to a response behavior that is generally more compliant than the elastic response, as the plastic response is superimposed on it. This scenario is very similar to the situation depicted in fig. 2.32, in which the elastic response is assumed as a base-line voltage [140]. The plastic response is due to small peaks in voltage in case of single events as observed in A), which evolve to avalanches in the response behavior due to increasing rate as depicted in B) and C).

The connection to the interval-based analysis performed and presented in section 5.4 is found in the sub-events of such an avalanche of plastic events as observed in fig. 2.32 C). Due to the fact that these sub-events are based on superposition of individual events of EIPmotif type, they are directly coupled to the intensity of plastic response. The power-law response in avalanche-dominated systems is assumed to be scale invariant, or at least close to scale-invariance [131]. The distribution of these sub-events or intervals should yield the characteristic form of a power law, as the case if the strain intensities of the cascades of plastic events are analyzed [140]. As the distributions on positive and negative events show, this is indeed the case, confirming this interpretation.

However, the behavior of the interval duration is somewhat unexpected in comparison to the reported behavior, which shows under increasing rate an increase of the duration for both individual plastic events like the EIP-motif [141, 77] and avalanches [140]. The fact that the duration is closely coupled to the strain rate as depicted in fig. 6.5. gives evidence that it is connected to the intrinsic plastic response of the material. Thus, it gives an indication that the intervals as defined for the incremental analysis in section 3.7 are not to be identified with individual plastic events, as the EIP-motif suggests. Neither do they reflect the full length of avalanches, which arise from the linear baseline as in fig. 2.32 B) and C). They refer to subsets of plastic processes, which reflect the scale invariant behavior typical for avalanche dominated systems [131]. As the number of individual processes contributing to these subsets is not known, a comparison on the duration of single events can not be given. 


\subsection{Comparison and Classification of Results from Nonlinear and Incremental Analysis}

In comparison, the nonlinear approach and the incremental approach for data analysis in case of LAOS-experiments yield very different results on the onset of plastic processes.

The nonlinear analysis provides evidence for a promoted nonlinearity only under high fields, while the sample response shows only a linear contribution under low field excitation. The proposed interpretation of linear and nonlinear behavior as the absence of rearranging events in the linear case and an activation of plastic behavior in the nonlinear case, however, is challenged by evidence by the incremental analysis that reveals an activation of plastic events for both high and low fields. This becomes evident by the power-law behavior in strain distributions extracted by incremental analysis, which is observed for all stress amplitudes investigated. This power-law observation indicates a connection of the measured strain to avalanches due to STZ $\$$ as observed in simulations [88, 32, 89, 137], colloidal experiments [122, 20, 19], as well as mechanical analysis [74, 54]. Thus, the regime of the stress-straincurve which is often referred to as linear or elastic in the Hookean sense is not purely elastic at all. It is connected to the appearance of $S T Z \$$. These are attributed to local rearrangements of plastic character, exhibiting a broad variation in the size of the triggered events due to the correlation of STZ $\beta$ via their elastic Eshelby field.

The enhanced strain response is reflected by the nonlinear contribution at fundamental frequency in case of the nonlinear analysis. This contribution yields a super-linear behavior and is depicted by the nonlinear analysis as an additional strain response to the linear response observed under low field excitation. The nonlinear contribution at the third harmonic frequency in contrast has a sub-linear effect on the compliance, i.e. reducing the compliance (e.g. observed under high intra-periodic stresses in fig. 5.1). The opposing effect of the nonlinear contribution at first and third harmonic frequency are both connected to the strain rate. In case of the first harmonic nonlinear contribution the effect is connected to an increase in strain rate, pushing the system further towards yielding. The other effect can be attributed to a dominating influence of a decrease in strain rate on the intra-periodic timescale, reflected in the nonlinear contributions of the third harmonic frequency. The connection of strain intervals to the strain rate is similarly observed in case of interval averages (fig. 6.5), confirming the interpretation regarding the third harmonic frequency. The strain rate has a strong influence on the averaged interval data at high intra-periodic stresses, exactly where the third harmonic frequency shows the strongest effect on the intra-periodic stress scale. An important question is, how the difference in low and high field response for the nonlinear analysis can be interpreted in connection to the results of the incremental analysis. The latter one shows a difference in the average interval strain which is connected to the strain rate via the stress amplitude. The magnitude of the averaged values is directly comparable to the linear and nonlinear response behavior of the nonlinear approach. However, the distributions of both low field and high field excitation show a power-law behavior, that indicates that for both excitation amplitudes the same response process is occurring. Even though the global response behavior does not directly reflect the connection to microstructural processes, the incremental approach clearly does as shown by the interval strain distributions. The response behavior is a result of the microscopic rearrangements termed as STZ $\$$, which even occur in case of low field excitation, i.e. low strain rate. It is connected to a response that appears to be elastic on the global scale, but is not on the local scale.

The comparison of the nonlinear and the incremental analysis and their results indicates a stronger sensitivity of the incremental analysis regarding the detection of plastic events. It becomes clear, that the extracted nonlinearity of the system is rather due to the global evaluation technique and not due to an intrinsic nonlinear character of compliance or modulus itself. It evolves from the averaging of the sample response over the range of one period 
by the period-by-period Fourier-analysis. It smoothens the response over the whole period neglecting its erratic character. Still, one of the advantages of the nonlinear analysis is the determination of the recovery timescale, which shows a connection to the structural relaxation time of the metallic glass. This connection of the overall sample response to the VFT-relation is a clear indicator for activated primary relaxation. This observation indicates that the applied high field excitation is sufficient to push the system towards the so-called anelastic part of the stress-strain-curve.

Even though the nonlinear analysis does not resolve the irreversible rearrangements occurring in the metallic glass samples to the same extent as the incremental analysis, it is a powerful tool at temperatures above the glass transition temperature in case of dielectric measurements. Here, the interval response is assumed to become less pronounced due to the enhanced superposition of avalanches. This intense avalanche-behavior eventually results in a more or less smooth data evolution, resulting in homogeneous deformation. It should highly depend on the data acquisition rate, to which extent intervals or avalanches can be resolved in this temperature regime. A smooth data evolution at these elevated temperatures is measured in case of dielectric experiments, avalanche-dominated behavior is not observed. The parallels of the results of nonlinear and incremental analysis reflect the general applicability of both methods. These are for example the enhanced strain response with increasing stress amplitude and increasing temperature, and the strain-rate dependence at high intraperiodic stresses. Still, the incremental approach disposes a higher sensitivity towards the occurrence of STZ $\beta$, even in the so-called elastic part of the stress-strain-curve. 


\subsection{Outlook}

A method for the incremental analysis of mechanical response data yielded by single cantilever bending experiments is successfully developed, which reveals the plastic response behavior with high sensitivity. However, this method could be extended regarding the evolution of distributions with intra-period stress and with time. The distributions shown in this thesis contain all intervals occurring in steady state periods. As long as the distributions include a sufficient amount of intervals to guarantee a sound statistical basis, the data can be subdivided. This way distributions can be extracted that depict the evolution with experimental time or which extract the change of the distribution with intra-periodic stress. Possibly, a better understanding of the hump could be gained, which might show a different appearance for data sub-sets connected to high strain rates.

Furthermore the interplay between stress amplitude and frequency has a strong influence on the values of strain rate. Its dominating influence on the sample response can be analyzed on a wider range by an extension of the applied frequency regime.

To learn about the occurrence of relaxation processes in other materials, further metallic glasses and polymers would be interesting candidates for evaluation. Data acquired for the nonlinear analysis of PMMA from the PhD-thesis by S. Finkhäuser is available for incremental analysis [44]. The master thesis by $C$. Garve even includes a comparison of the nonlinear and incremental analysis on metallic glass $\mathrm{Pd}_{77.5} \mathrm{Cu}_{6.0} \mathrm{Si}_{16.5}$ samples [47].

Also, an extension of the regime of the experimental parameters is desired. These are the temperature $T$, stress amplitude $\sigma_{0}$, and frequency $f$. At very low temperatures a change of the response behavior is expected, as the intensity of avalanche-behavior should subside. This is possibly resulting in the observation of avalanches and sequences of linear behavior, as depicted in fig. 2.32 B) and also by the EIP-motif.

Apart from an extended parameter range, the type of excitation could be changed from sinusoidal to a saw-tooth excitation. The influence of strain rate on the intra-periodic stress scale would be avoided as the strain rate is constant, resulting in a simpler experimental situation in case of the incremental analysis. The nonlinear analysis could be performed still, as the saw-tooth excitation corresponds to a superposition of odd-harmonic frequencies. Still, the data evaluation becomes more complicated, as the response signal regarding the expected response frequencies overlaps with the spectrum of excitation frequencies.

In case of the nonlinear analysis, measurements at further frequencies might reveal the evolution of the number of correlated particles. Materials with lower modulus might be more applicable than metallic glasses as these generate a strong resonance at higher frequencies due to their relatively high modulus in experiments with the DMA 8000. An adjustment of the sample geometry by further reduction of the sample width and thickness might improve the resonance issues as well. This would also suit the idea of an excitation with higher stress amplitudes without implications by nonlinear contributions of the machinery.

In conclusion, the mechanical analysis on the basis of LAOS-measurements contributes to the understanding of the response behavior of metallic glasses as it reveals the existence of avalanche dynamics in the so-called elastic or linear response regime of the stress-strain-curve. Still, open questions especially in connection to the origin of the hump in the distribution of interval strain remain. By further development of the experimental approach as elaborated above, the investigation of this phenomenon could be pursued by the proposed incremental analysis. 



\section{Acknowledgment}

First of all I want to thank Prof. Konrad Samwer, who gave me the opportunity to develop my thesis under his supervision. He fully supported me from the very first moment, when I entered his office to apply for a PhD-position in his group. He offered his knowledge and experience to learn about the field of amorphous matter, and to explore own ideas and interpretations. He often discussed with me and gave his opinion on numerous questions and ways of interpretation. I am very grateful that he accepted me as his student, as his scientific guidance influenced me and helps me not only in regard to my thesis, but also for my future scientific development.

During my thesis I was allowed to gain experience on several national and international conferences and got the freedom to grow on the challenges that were offered to me. Especially the projects at Arizona State University in the United States of America with Prof. Ranko Richert, and the project at the Weizmann Institute of Science in Israel with Prof. Itamar Procaccia learned me a lot, both from the physics point of view, and in terms of personal experiences. Thus, I want to say a special thanks to Ranko and Itamar, who impressed me a lot personally and scientifically, each in their very individual ways.

Furthermore, I want to thank Prof. Hans-Ulrich Krebs who agreed on being the second supervisor of my thesis, and to Prof. Cynthia Volkert, who willingly accepted on being the second referee for the evaluation of my thesis.

Prof. Dr. Vasile Mosneaga, Prof. Dr. Michael Seibt, Dr. Richard Vink, and Dr. Claus Heussinger are appreciated members of my thesis committee and I want to thank all of them for their straightforward attitude when it came to the question if they would evaluate my thesis.

Another important acknowledgment goes to the financial support by the DFG by the Research Unit 1394 on 'Nonlinear Response and Probe Vitrification'. Prof. Matthias Fuchs and Marianne Griesser are acknowledged as they supported my application and helped me with many questions regarding the funding for the projects in the US and Israel by the MINprogram. I am very lucky that I can say that I have never been in danger of running out of financial support, and that I got so extensive support for traveling expanses.

I really appreciate the amiable atmosphere at the I. Physics Department and the very pleasant interactions with its members. In the glass group we always had a good time at the institute, and I will keep many precious memories on the times we spent on conferences together. Long discussions on avalanche theory and the important things in life with Alexandra will be especially missed, when our ways will finally split. At this point I also want to say thank you to my office mates. Büchse, who always had some good advise when I was struggling with my data. Steffi, who has a similarly giggly way as I have when it comes to the point of mental depletion. Clemens, who is reliable and helpful when help is really needed. I am very happy that he was insistent to start his master thesis in the group of Prof. Samwer.

When it came to the handling of experimental setups and the preparation of samples, especially Carsten Mahn, Uta Filippich, Katrin Gehrke, and Dennis Kohl gave their intense support. Due to their comforting and cooperative way, I was never in doubt about the fulfill- 
ment of experimental tasks. At this point I also want to mention the appreciated sharing of a bedroom in Klosters with Uta, despite all the threats on nightly background noises. Who knows if I would have had that much fun on my snowboard in the very first year, if she hadn't pulled me along all those cat tracks.

I also want to thank the workshop of the I. Physics department, and the central workshop for their help, which was always given when needed.

I also want to say special thanks to the girls of my Volleyball team who always provided me with excellent distraction when work was stressful and didn't want to get out of my head by itself. And for sure, I will stay tuned on the latest unicorn trends. I also enjoyed the days I went for running with Volker and Johanna, that gave me a good balance and which eventually changed to coffee-pepp-talks. My friend Babsa is gratefully mentioned for her support by squash sessions, wine, and encouraging words.

Very special thanks go to my family, who supported me and strengthened me throughout my entire studies. Their conviction that I would accomplish, whatever aim I set to myself, made me believing it too. The loving words by my mother, the logical approach of problems by my father, the reflections and sympathy by my sister, paintings with cats in capes by my nieces, and the pride and fondness by my grandparents.

I want to express my deepest gratitude towards Tobi, who gave me incredible support over the past years. He has to endure all the struggles I go through and makes me laugh when I really don't feel like it, but need it the most. You always give me the help and support I ask for, and I owe you for allowing me to focus on my work and to realize myself. Ich danke Dir. 


\section{Appendix A}

\section{Calibration of the DMA 8000}

In this chapter the principles of temperature, force, and spring stiffness calibration are described. Moreover the influence of the thermal expansion of the movable axis of the DMA is quantitatively discussed regarding its influence on LAOS-measurements.

The temperature calibration of the DMA 8000 is performed by temperature scans on Indium and Lead, separately, by determination of the onset of melting. Therefore, small amounts of the material are placed into a material's pocket and the overall modulus of pocket and calibration material is measured. This calibration should be renewed every quarter of a year or at least every year.

The calibration of force amplitude is necessary to determine the voltage that is needed to apply a specific force to the sample. For calibration, a balance weight of known mass is used in vertical head orientation, the position of the drive shaft measured by the [DDT is used to determine the force necessary to re-balance the movable axis with the additional weight. This calibration should be performed every six month or at least once a year.

Another important quantity for determination of correct force values is the spring stiffness and damping of the force motor. According to a Perkin Elmer the force motor contains a system of eight springs. These springs and the force motor itself dispose a certain stiffness, which becomes larger with increasing displacement of the movable axis from its zero position. This calibration is useful to determine the acting forces due to spring stiffness to estimate the displacement value for which an impact of the spring stiffness on the experiment becomes significant. This calibration should be performed on similar intervals as the force amplitude calibration.

For the acquisition of data presented in this thesis the same temperature calibration was applied to all experiments. Force amplitude and spring stiffness calibration was used on temperature scans, a correction during LAOS-experiments is not applicable. Therefore, assumptions on the impact of external forces, e.g. due to the spring stiffness and damping of the machine are made. Force and displacement amplitudes during LAOS-experiments are held within a range of negligible impact based on these calculations.

The calibration for spring stiffness and damping gives a relation between displacement amplitude $k$ and counter-acting forces $F$ by the force motor, see fig. A.1. The exact relation is given by a fit with a polynomial function

$$
F_{\text {tot }}=S_{0} \cdot k-S_{1} \cdot k^{2}+S_{2} \cdot k^{3}+S_{3} \cdot k^{4}-S_{4} \cdot k^{5}-S_{5} \cdot k^{6}+S_{6} \cdot k^{7},
$$

with the linear part of the force $F_{l i n}=S_{0} \cdot k$.

The absolute values of the counter-acting force due to spring stiffness and damping are given in fig. A.1a both for the total force $F_{\text {tot }}$ and the linear part $F_{\text {lin. }}$. For displacements achieved in the LAOS-experiments, which are at $45 \mu \mathrm{m}$ at maximum, the counter acting forces of the machine are in the order of maximal $0.097 \mathrm{~N}$ corresponding to $3.9 \%$ to $6.5 \%$ of the force applied during the experiment depending on temperature. In fig. A.1b the nonlinear part of the counter-acting force relative to its linear part $F_{l i n}$ is plotted, which is of interest as the 
nonlinearity of the counter-acting force might result in a nonlinear excitation signal, even though the technically applied force is linear. The impact of the nonlinearity of the counteracting force $F_{\text {tot }}$ depends on the displacements achieved during experiment, thus the amount of nonlinearity is in the order of $0.15 \%$. This results in a relative nonlinearity in force of $9.75 \times 10^{-5}$ at maximum relative to the applied force, which is two orders of magnitude lower than the relative value of the highest harmonic contribution of the Fourier-transformed LAOS-data. Thus it should not show a significant impact on the results of the analysis.

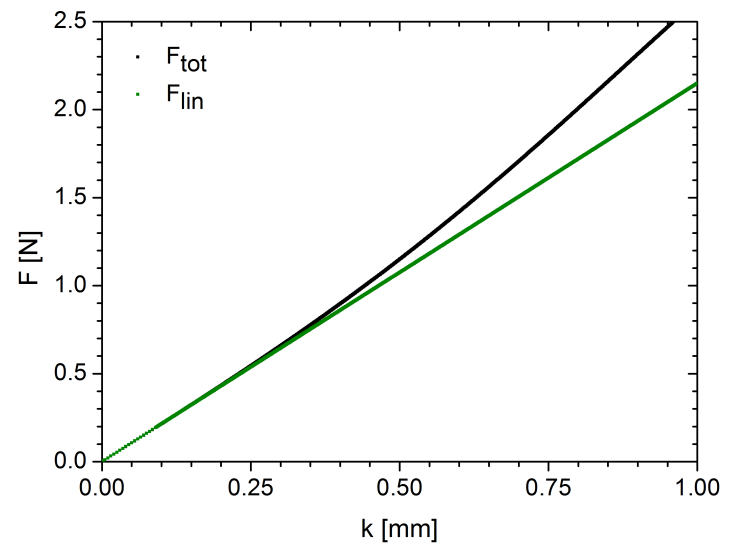

(a) Total (black) and linear (green) part of counter-acting force $F$ plotted against displacement $k$.

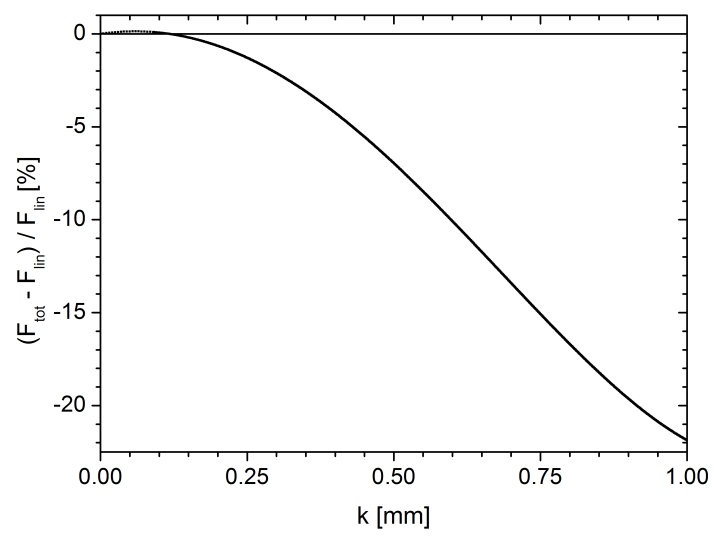

(b) Relative nonlinear part of counter-acting force $F$ plotted against displacement $k$.

Figure A.1 Counter-acting force due to spring stiffness and damping $F$

An aspect which shows an influence on the displacement offset is the thermal expansion of the movable axis. As the zero-force calibration is performed at ambient temperature, it does not take into account the change in length due to the heating of the sample chamber. Under the assumption that the temperature of the movable axis equilibrates at a comparable temperature as the sample, a temperature difference of at least $250 \mathrm{~K}$ has to be taken into account. For a movable axis with a length of roughly $10 \mathrm{~cm}$ made of Titanium with a linear expansion coefficient of $8.6 \times 10^{-6} \mathrm{~K}^{-1}$ [53] the change in axis length can be calculated by [30]

$$
\Delta L=\alpha L \Delta T,
$$

which yields a change of roughly $215 \mu \mathrm{m}$ or even more if the temperature dependence of the linear expansion coefficient $\alpha$ is taken into account. Experimentally, a change in the static displacement of 65 to $85 \mu \mathrm{m}$ is found, which is lower than the value estimated due to thermal expansion. This result implies that the effect of thermal expansion plays a significant role for the offset position of the movable axis. Even if a more accurate positioning of the movable axis during the mounting of the sample were applied, the expansion with temperature would still affect the accuracy of LAOS-measurements in terms of a changing offset position. A change in the offset during the experiment could result in an artificial phase shift between different harmonics, and the forces implied by the offset might even affect the nonlinear behavior of the material. By sufficient equilibration at elevated temperature, a change in the offset due to thermal expansion of the movable axis is negligible, however, the absolute value of the offset remains. 


\section{Appendix B}

\section{Geometry Factor}

An important point is the estimation of the geometry factor $b$, which is the relation between force $F$ and displacement $k$. As the sample is clamped on both sides, both bending and stretching contribution have to be taken into account, if large deflections are assumed [128]. This leads to a relation between force and displacement which reads:

$$
F=\left(\frac{\pi}{2}\right)^{4} \frac{E W H}{L_{0}^{3}} \cdot k\left(\frac{H^{2}}{3}+\frac{k^{2}}{4}\right)
$$

with Young's modulus $E$, width $W$, thickness $H$, and initial length $L_{0}$. If the bending term is much larger than the stretching term, $\frac{H^{2}}{3}>>\frac{k^{2}}{4}$, the relation between force and displacement can be assumed as linear. For maximum values in displacement $k$ of $45 \mu \mathrm{m}$ and a typical sample thickness of $H=1 \mathrm{~mm}$ the relation holds with:

$$
\frac{H^{2}}{k^{2}} \frac{4}{3}=\frac{(1 \mathrm{~mm})^{2}}{(45 \mu \mathrm{m})^{2}} \frac{4}{3}=660>>1
$$

Thus, a linear relation can be assumed and the validity of the description used for calculation of stress and strain values is given, as the nonlinear stretching term is by more than two orders of magnitude smaller than the linear bending term in case of the highest resulting strain amplitude. 


\section{Appendix C}

\section{Sinusoidal Excitation Including Offset}

In case an offset due to clamping, thermal expansion of the movable axis, or misalignment of the system occurs, the offset itself might inflict nonlinear contributions at odd as well as even frequencies. To test for influences of the always existing offset, the dependence of the displacement signal on the these contributions needs to be evaluated. These contributions evolve as follows due to an additional offset $\sigma_{s t}$ in stress:

$$
\hat{\sigma}=\sigma_{0} \cdot \sin (\omega t)+\sigma_{s t}
$$

With eq. 3.16 this yields:

$$
\begin{array}{r}
\hat{\epsilon} / \sigma_{0}=\hat{\chi}_{0}^{(1)} \frac{\sigma_{s t}}{\sigma_{0}}+\hat{\chi}_{0, a}^{(3)} \frac{\sigma_{s t}^{3}}{\sigma_{0}}+\frac{3}{2} \hat{\chi}_{0, b}^{(3)} \sigma_{s t} \sigma_{0}+\hat{\chi}_{0, a}^{(5)} \frac{\sigma_{s t}^{5}}{\sigma_{0}}+5 \hat{\chi}_{0, b}^{(5)} \sigma_{s t}^{3} \sigma_{0}+\frac{3}{8} \hat{\chi}_{0, c}^{(5)} \sigma_{s t} \sigma_{0}^{3} \\
+\left(\hat{\chi}^{(1)}+\frac{3}{4} \hat{\chi}_{1}^{(3)} \sigma_{0}^{2}+3 \hat{\chi}_{1, a}^{(3)} \sigma_{s t}^{2}+\frac{5}{8} \hat{\chi}_{1}^{(5)} \sigma_{0}^{4}+\frac{15}{2} \hat{\chi}_{1, a}^{(5)} \sigma_{0}^{2} \sigma_{s t}^{2}+5 \hat{\chi}_{1, b}^{(5)} \sigma_{s t}^{4}\right) \cdot \sin (\omega t) \\
+\left(\frac{3}{2} \hat{\chi}_{2}^{(3)} \sigma_{0} \sigma_{s t}+5 \hat{\chi}_{2, a}^{(5)} \sigma_{0} \sigma_{s t}^{3}-\frac{1}{2} \hat{\chi}_{2, b}^{(5)} \sigma_{0}^{3} \sigma_{s t}\right) \cdot \cos (2 \omega t) \\
+\left(-\frac{1}{4} \hat{\chi}_{3}^{(3)} \sigma_{0}^{2}-\frac{5}{16} \hat{\chi}_{3}^{(5)} \sigma_{0}^{4}-\frac{5}{2} \hat{\chi}_{3, a}^{(5)} \sigma_{0}^{2} \sigma_{s t}^{2}\right) \cdot \sin (3 \omega t) \\
+\frac{1}{8} \hat{\chi}_{4}^{(5)} \sigma_{s t} \cos (4 \omega t) \\
+\frac{1}{16} \hat{\chi}_{5}^{(5)} \sigma_{0}^{4} \sin (5 \omega t)
\end{array}
$$

If terms of orders higher than three are neglected the expression is reduced to:

$$
\begin{array}{r}
\hat{\epsilon} / \sigma_{0}=\hat{\chi}_{0}^{(1)} \frac{\sigma_{s t}}{\sigma_{0}}+\hat{\chi}_{0, a}^{(3)} \frac{\sigma_{s t}^{3}}{\sigma_{0}}+\frac{3}{2} \hat{\chi}_{0, b}^{(3)} \sigma_{s t} \sigma_{0} \\
+\left(\hat{\chi}^{(1)}+\frac{3}{4} \hat{\chi}_{1}^{(3)} \sigma_{0}^{2}+3 \hat{\chi}_{1, a}^{(3)} \sigma_{s t}^{2}\right) \cdot \sin (\omega t) \\
+\frac{3}{2} \hat{\chi}_{2}^{(3)} \sigma_{0} \sigma_{s t} \cos (2 \omega t) \\
-\frac{1}{4} \hat{\chi}_{3}^{(3)} \sigma_{0}^{2} \sin (3 \omega t)
\end{array}
$$

If it is possible to show that terms of 5th order don't have measurable impact on the interpretation of the results, the impact of the offset might be tested by plotting of the second harmonic amplitude divided by the maximum stress amplitude $\sigma_{0}$ against the stress offset $\sigma_{s t}$. If a linear dependence on $\sigma_{s t}$ can be excluded, the stress offset should not have an impact on the analysis of the results of the experiment. 
Fig. C.1 depicts the linear dependence of the second harmonic frequency contribution on the static stress $\sigma_{s t}$ as stated in eq. C.3. It is negligible for temperatures of $566 \mathrm{~K}$ or less, while for the data measured at a temperature of $567 \mathrm{~K}$ a linear dependence is observed in fig. C.1. Thus, measurements at temperatures closer to the glass transition should be carefully analyzed towards the influence of the static offset, which also results in a contribution at fundamental frequency. Only if this contribution is comparably small, the nonlinear analysis yields reliable nonlinear coefficients at the fundamental frequency.

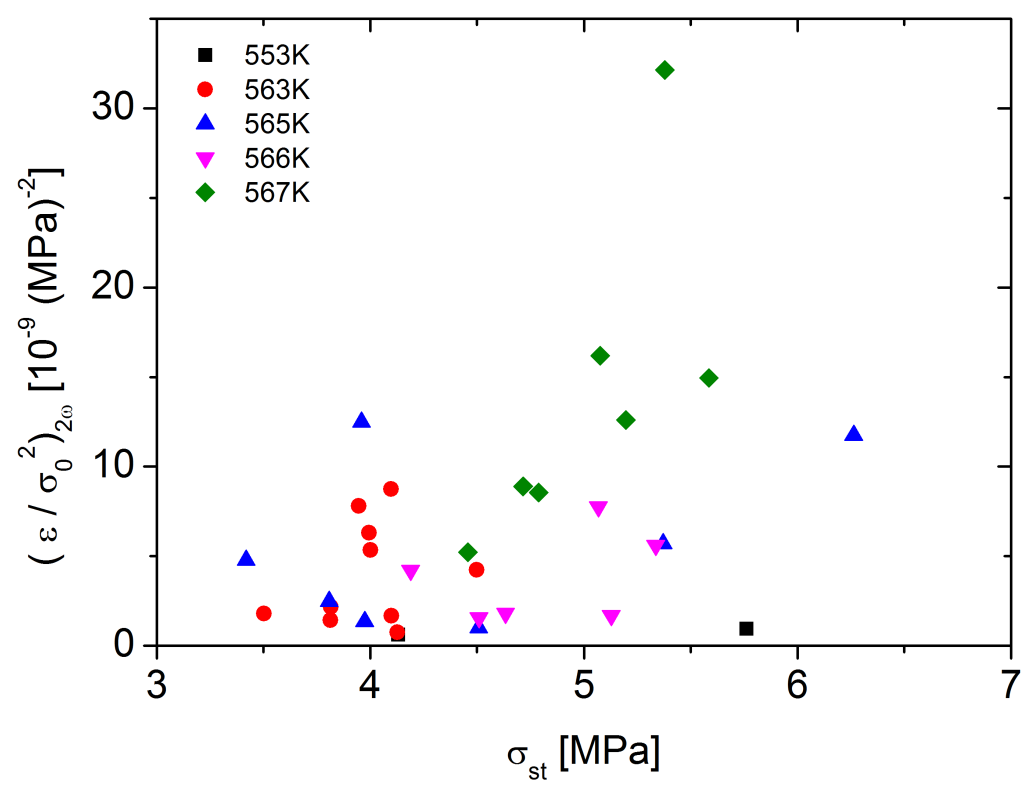

Figure C.1 Second harmonic strain amplitude divided by squared stress amplitude $\left(\varepsilon / \sigma_{0}^{2}\right)_{2 \omega}$ plotted against static stress $\sigma_{s t}$ for different temperatures. 


\section{Appendix D}

\section{Fifth Harmonic Frequency Response}

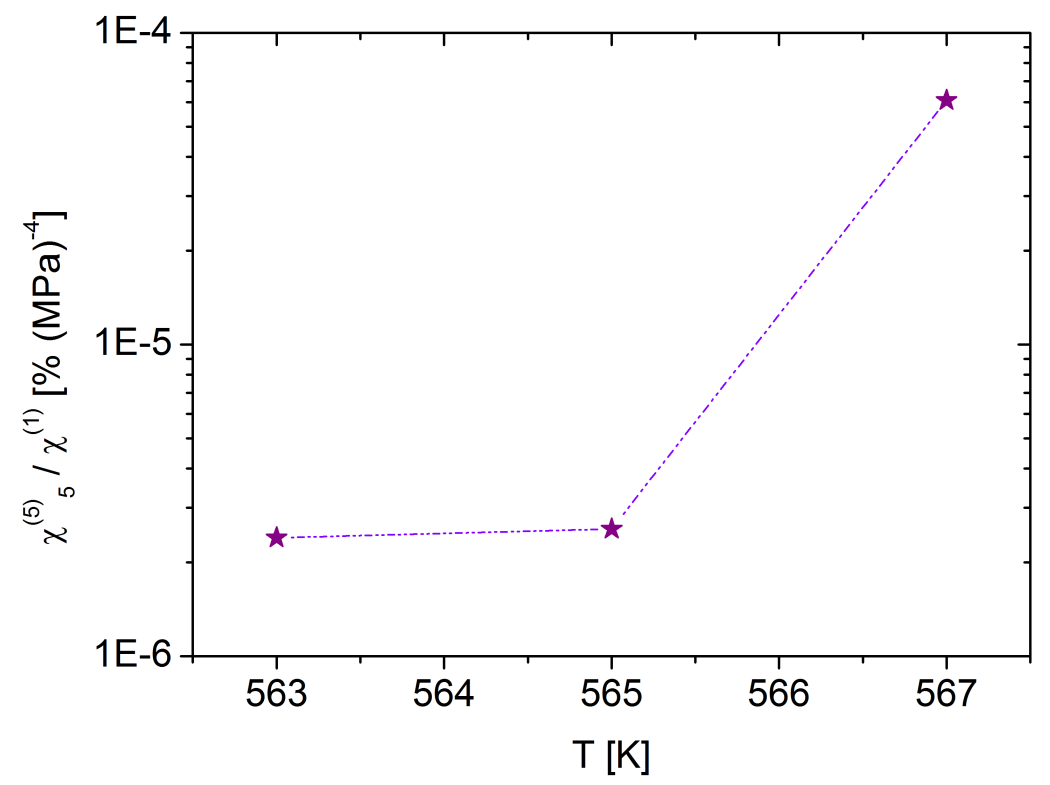

Figure D.1 Relative nonlinear coefficient $\chi_{1}^{(5)} / \chi^{(1)}$ plotted in logarithmic scaling against temperature $T$.

Few measurements show a fifth harmonic contribution, which is the basis for the analysis of the nonlinear coefficient as shown in fig. D.1. As very few measurements contribute to the extraction of the coefficient, it is reliable, but is rather giving an example on the trend observed for this quantity, which is increasing with increasing temperature. The value of the fifth harmonic relative nonlinear coefficient is about three orders smaller than the relative nonlinear coefficients extracted for the first and third harmonic frequency. 


\section{Appendix E}

\section{MatLab-Routines for Incremental Analysis}

\section{E.1 Definition of Increments}

The code presented in this section is based on the stress and strain data in the units MPa and $\%$, which are given in the text files 'data-StrainPercent-StressMPa.txt'. The praefix 'data' specifies the measurement which is to be evaluated. The text file contains two columns of $1000 \times 3600 \times 3=1.08 \times 10^{6}$ rows each, the first column contains the stress data and the second the strain data with an accuracy of 1000 data points per period. The code generated increments, which are the basis for the extraction of intervals, as described in section 3.7

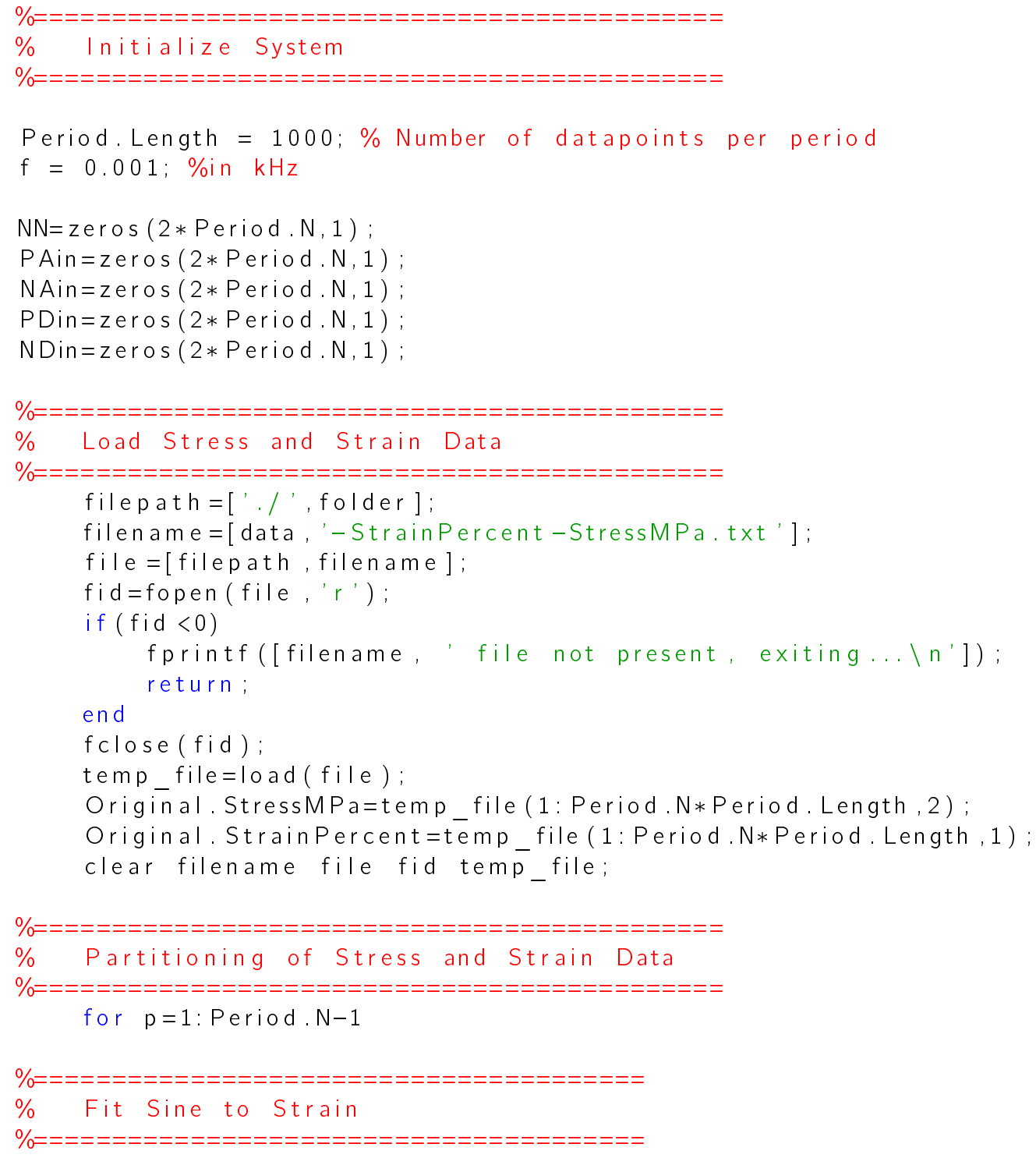


$x=1: 1: 2000 ; \%$ in ms

$y=$ Original. StrainPercent $(1+(p-1) *$ Period. Length $: 2000+(p-1) *$ Period . Length ):

$\mathrm{yu}=\max (\mathrm{y})$;

$\mathrm{yl}=\min (\mathrm{y})$;

$y r=(y u-y l) ; \quad$ \%Range of ' $y$ '

$\mathrm{ym}=\operatorname{mean}(\mathrm{y})$;

fun $=@(r) r(1) \cdot *\left(\sin \left(2 * p i * f * x^{\prime}+r(2)\right)\right)+r(3)-y$;

$\mathrm{x} 0=[\mathrm{yr} / 2,0, \mathrm{ym}] ;$

$r=$ Isquonlin (fun, $x 0)$;

Fit. $r 1(p)=r(1)$;

Fit. $r 2(p)=r(2)$;

Fit. $r 3(p)=r(3)$;

Fit.fitr $=$ Fit. $r 1(p) *(\sin (2 * p i * f * x+F i t . r 2(p)))+F i t \cdot r 3(p)$;

clear yu yl yr ym fun $r \times 0 \times y$

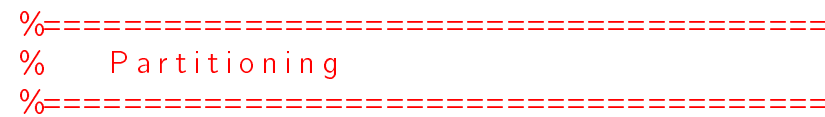

$N N(p)=$ find (Fit. fitr $(1,1$ : Period. Length $)==$ min (Fit. fit $r(1,1$ : Period .

Length))); \%defines minimum of each period

if $N N(p)>=$ Period. Length $* 3 / 4+1$

PAin $(p)=N N(p)+$ Period. Length $/ 4-$ Period. Length; \%begin positive ascending part of the period

else

PAin $(p)=N N(p)+$ Period. Length $/ 4$; \%begin positive ascending part of the period

end

Period. PosAscStrain $(:, p)=$ Original. StrainPercent (PAin $(p)+(p-1) *$

Period. Length: PAin $(p)+$ Period. Length $/ 4-1+(p-1) *$ Period. Length , 1$)-$

Original. StrainPercent (PAin $(p)+(p-1) *$ Period. Length, 1$)$; \%um Offset korrigiert in jeder Periode

Period. PosAscStress $(:, p)=$ Original. StressMPa (PAin $(p)+(p-1) *$ Period .

Length: PAin ( $p)+$ Period. Length $/ 4-1+(p-1) *$ Period . Length , 1$)$;

if $N N(p)>=$ Period. Length $/ 2+1$

PDin $(p)=N N(p)+$ Period . Length $/ 2-$ Period. Length; \%begin positive descending part of the period

else

PDin $(p)=N N(p)+$ Period. Length $/ 2$; \%begin positive descending part of the period

end

Period. PosDescStrain $(:, p)=$ Original. StrainPercent (PDin $(p)+(p-1) *$

Period. Length: PDin ( $p)+$ Period. Length $/ 4-1+(p-1) *$ Period. Length , 1$)-$

Original. StrainPercent (PAin $(p)+(p-1) *$ Period. Length 1 ); \%um Offset korrigiert in jeder Periode

Period. PosDescStress $(:, p)=$ Original. StressMPa (PDin $(p)+(p-1) *$ Period

Length: PDin $(p)+$ Period. Length $/ 4-1+(p-1) *$ Period. Length , 1$)$;

if $N N(p)>=$ Period. Length $* 1 / 4+1$

NDin $(p)=N N(p)+$ Period. Length $* 3 / 4-$ Period. Length; \%begin negative descending part of the period

else

$\operatorname{NDin}(p)=N N(p)+$ Period. Length $* 3 / 4 ; \%$ begin negative descending part of the period

end

Period. NegDescStrain $(:, p)=$ Original. StrainPercent $(\operatorname{NDin}(p)+(p-1) *$ Period. Length: NDin ( $p)+$ Period. Length $/ 4-1+(p-1) *$ Period. Length , 1$)-$ Original. StrainPercent (PAin $(p)+(p-1) *$ Period. Length, 1$)$; \% Offset korrigiert in jeder Periode

Period. NegDescStress $(:, p)=$ Original. StressMPa (NDin $(p)+(p-1) *$ Period Length: NDin ( $p)+$ Period. Length $/ 4-1+(p-1) *$ Period. Length , 1$)$;

NAin $(p)=N N(p)$; \%begin negative ascending part of the period 
$$
89
$$

$$
0 \%
$$




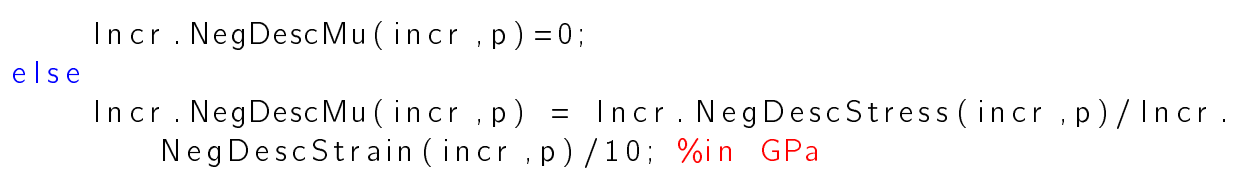

\section{E.2 Definition of Intervals}

The following code is based on the Matlab-files including 'Period' and 'Incr' data, which are generated by the code given in section E.1 (Definition of Increments). It defines positive and negative intervals according to the definition in section 3.7 .

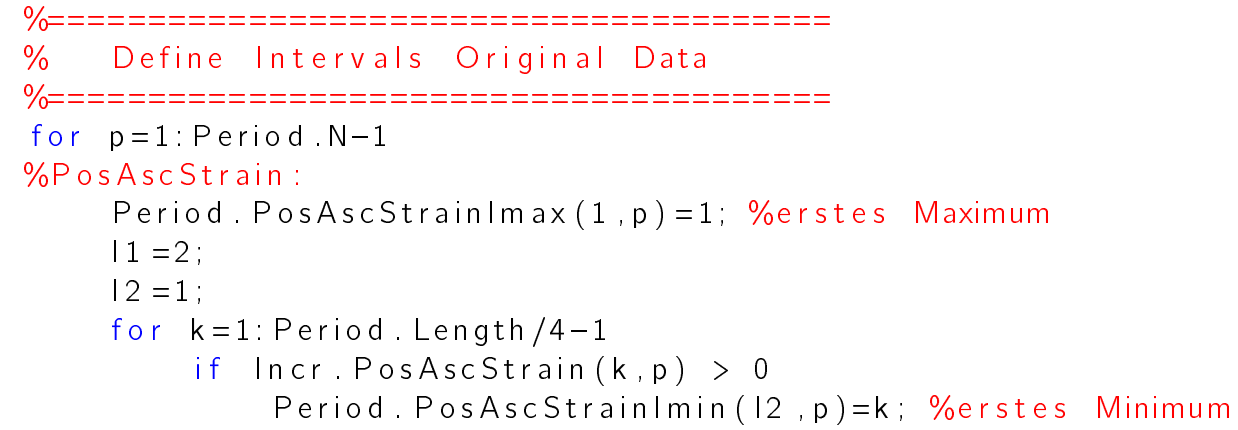


if Period. PosAscStrainImin (I2, p) ==Period. PosAscStrainImax (I1 $-1, p$ )

Period. PosAscStrainlmin $(12, \mathrm{p})=0$

Period. PosAscStrain Imax $(I 1-1, p)=k+1$; continue ;

end

$12=12+1$

Period. PosAscStrain Imax $(I 1, p)=k+1$;

$I 1=\mid 1+1$;

end

end

if $12==1$

end

Period. PosAscStrainlmin ( $12, \mathrm{p})=$ length (Period.PosAscStrain $(:, \mathrm{p}))$;

Period. PosAscStrainltvl $(\mathrm{p}, 1)=$ nnz(Period. PosAscStrainlmin $(:, p))$; clear l1 l2 $\mathrm{k}$

for itvI =1:nnz(Period. PosAscStrainImax $(:, p)$ ) $-1 \%$ for each interval |tvl. PosAscStrainPMean(itvl, p) = mean(Period. PosAscStrain (Period. PosAscStrain Imin (itvl, p) : Period. PosAscStrainlmax (itvI +1,p),p) ); \% Mittelwert des Intervalls (Dehnung)

|tvI. PosAscStrainPInitial (itvl, $p$ ) = Period. PosAscStrain (Period PosAscStrainImin (itvl, p), p); \% Anfangswert des Intervalls ( Dehnung)

ItvI. PosAscStrainPDelta (itvl, $p$ ) = Period. PosAscStrain (Period. PosAscStrainImax (itvI $+1, p), p$ )-Period. PosAscStrain (Period. PosAscStrainlmin (itvl , p), p); \% Intervall (Dehnung)

ItvI. PosAscStrainPDelta2 (itvl, p) $=\operatorname{sum}(\mid \mathrm{ncr}$. PosAscStrain (Period. PosAscStrainImin (itvl, p) : Period. PosAscStrainImax (itvI +1,p) -1, p) ) ;

ItvI. PosAscStrainPLength (itvl, p) = Period. PosAscStrainImax (itvI $+1, p)$-Period. PosAscStrainlmin (itvl , p); \% Datenpunkte des Intervalls

$|t v|$. PosAscStrainPMeanStress (itvl, p) = mean(Period. PosAscStress ( Period. PosAscStrainImin (itvl, p) : Period. PosAscStrainlmax (itvl $+1, p), p)) ; \%$ Mittelwert des Intervalls (Spannung)

$\mid$ tv I. PosAscStrainPInitialStress (itvl, $p)=$ Period. PosAscStress ( Period. PosAscStrainlmin (itvl, p),p); \% Anfangswert des Intervalls (Spannung)

$|t v|$. PosAscStrainPDeltaStress (itvl, $p$ ) = Period. PosAscStress (Period. PosAscStrainlmax (itvI $+1, p), p)$-Period. PosAscStress (Period. PosAscStrainlmin (itvl , p), p) ; \% Intervall (Spannung)

ItvI. PosAscStrainNMean (itvl, p) = mean(Period. PosAscStrain (Period. PosAscStrainlmax (itvl, p): Period. PosAscStrainImin (itvl, p) , p)) ;

ItvI. PosAscStrainNInitial (itvI, $p$ ) = Period. PosAscStrain (Period. PosAscStrainlmax (itvl, p), p);

$|t v|$. PosAscStrainNDelta (itvl, $p)=$ Period. PosAscStrain (Period. PosAscStrainImin (itvl, p) , p)-Period. PosAscStrain (Period. PosAscStrainlmax (itvl, p), p);

$|t v|$. PosAscStrainNDelta2 (itv|, p) $\operatorname{sum}(\mid \mathrm{ncr}$. PosAscStrain (Period PosAscStrainlmax (itvl, p) : Period. PosAscStrainImin (itvl, p) - $1, p$ ) ) ;

$|t v|$. PosAscStrainNLength (itvl, $p)=$ Period. PosAscStrain|min (itvl, $p$ )-Period. PosAscStrainImax (itvl, p);

$|t v|$. PosAscStrainNMeanStress (itvl, p) = mean(Period.PosAscStress ( Period. PosAscStrainImax (itvl, p) : Period. PosAscStrainImin (itvl, p), $\mathrm{p}))$;

ItvI. PosAscStrainNInitialStress (itvl, $p)=$ Period. PosAscStress ( Period. PosAscStrainlmax (itvl, p), p);

ItvI. PosAscStrainNDeltaStress (itvl, p) = Period. PosAscStress ( Period. PosAscStrainlmin (itvl, p) , p)-Period. PosAscStress (Period PosAscStrainlmax (itvl, p), p);

$|t v|$. PosAscStrainPChi (itvl, $p)=(|t v|$. PosAscStrainPDelta (itvl, $p) \mid$ ItvI. PosAscStrainPDeltaStress (itvl, p)) *10;

if isinf(|tv|. PosAscStrainPChi(itvl,p))==1 


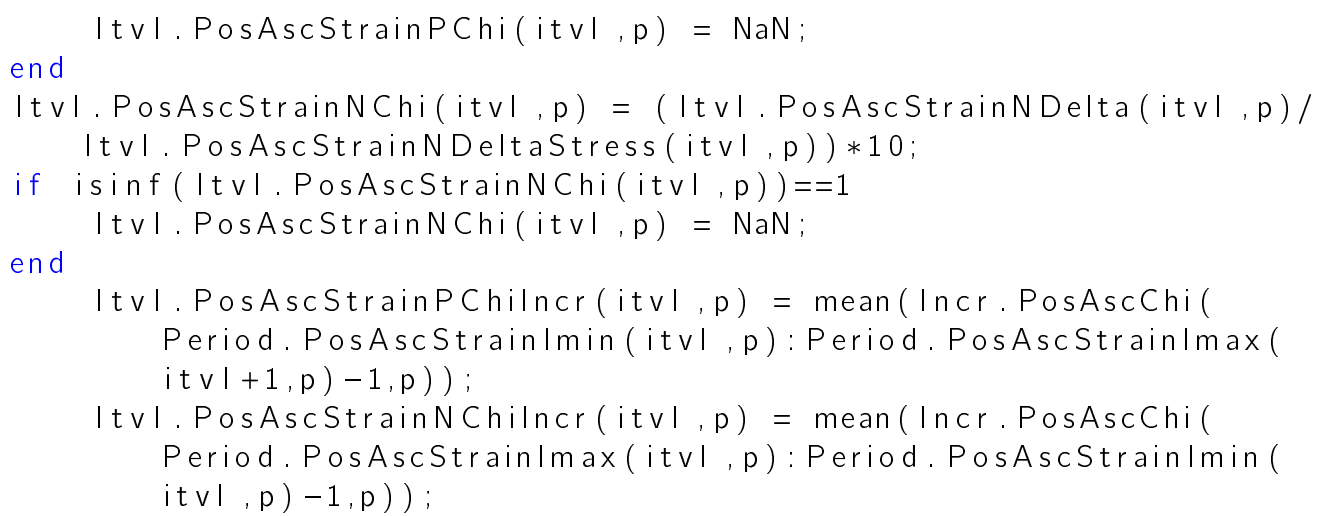

\section{E.3 Subdivision to Stress-Windows}

The following code is based on the Matlab-files including 'Period' and 'Itvl' data, which are generated by the code given in section E.2 (Definition of Intervals). It subdivides the interval data into stress windows of widths $\mathrm{cW}$, which is set to $2 \mathrm{MPa}$ for the presented analysis on averaged interval data.

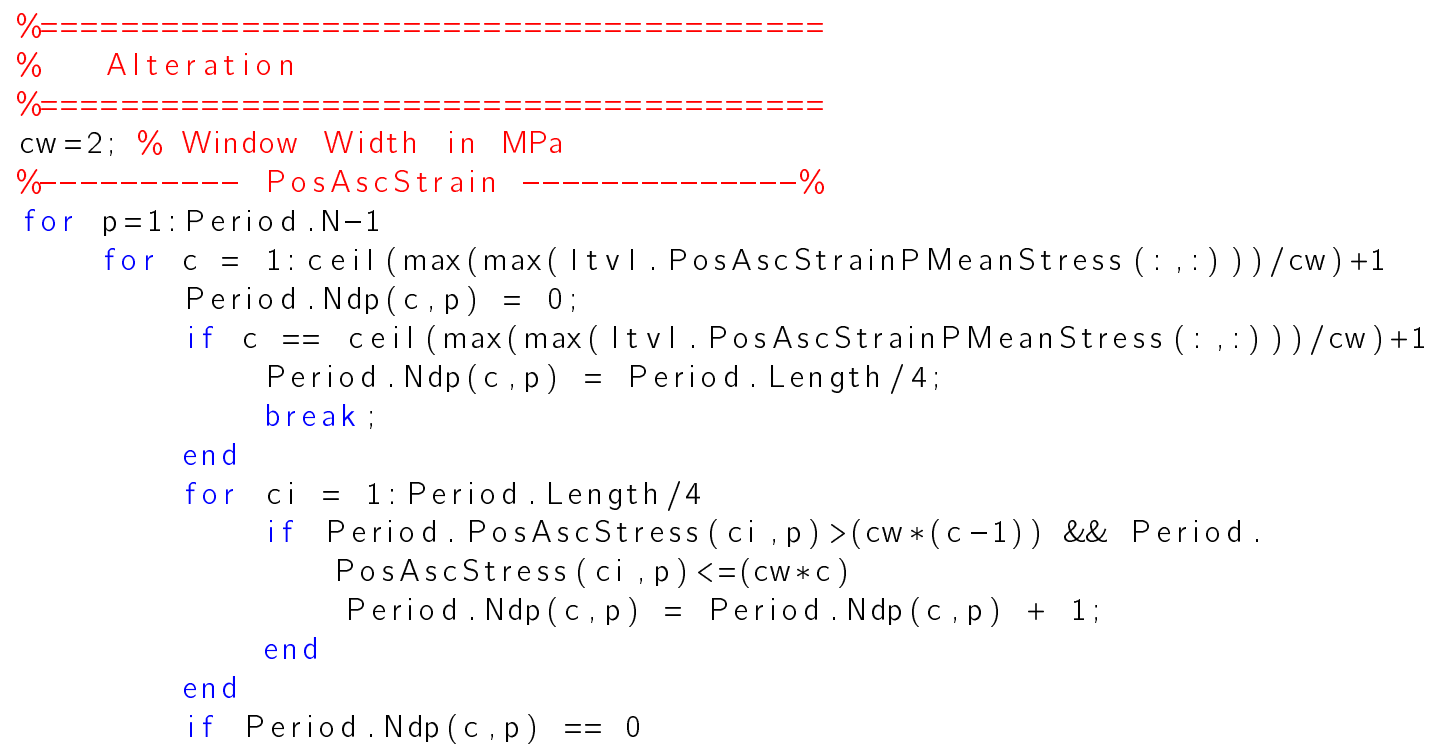




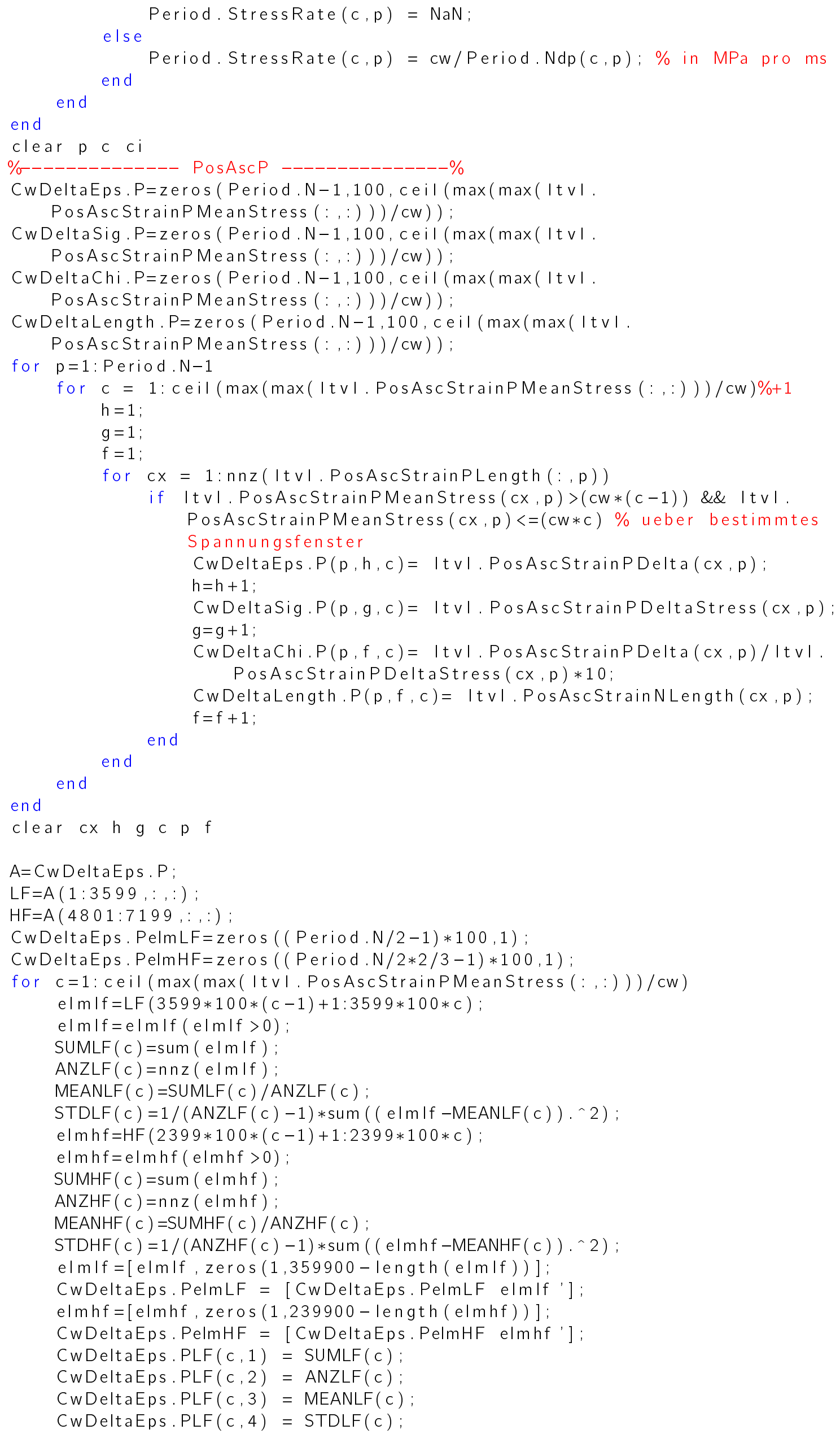




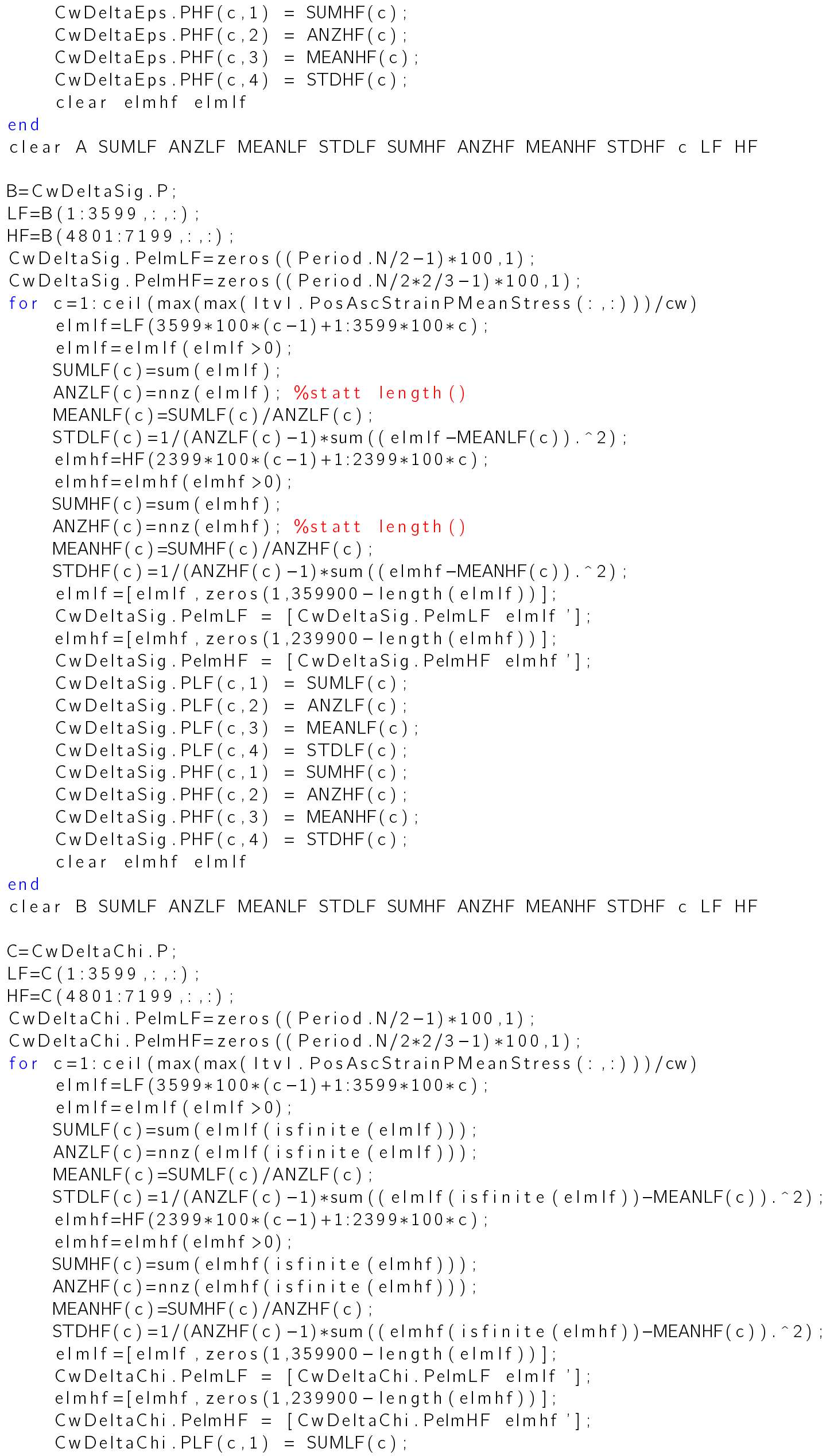




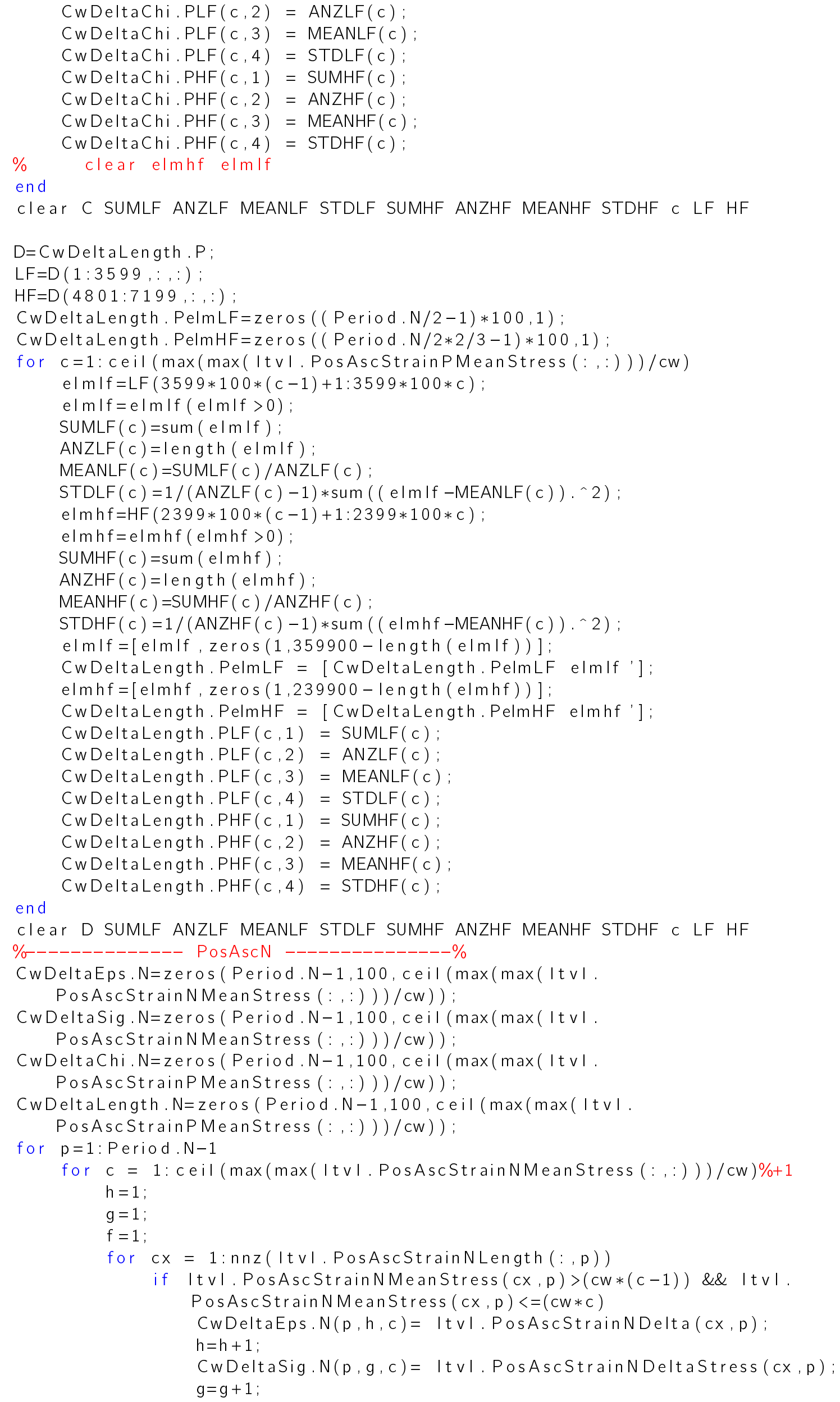


CwDeltaChi.N $(p, f, c)=|t v|$. PosAscStrainNDelta $(c x, p) /|t v|$.

PosAscStrainNDeltaStress ( $c x, p) * 10$;

CwDeltaLength. $N(p, f, c)=|t v|$. PosAscStrainNLength $(c x, p)$; $f=f+1$; end end

end

end

clear h g c cx p f

$\mathrm{A}=$ CwDeltaEps. N;

$\mathrm{LF}=\mathrm{A}(1: 3599,:,:)$;

$\mathrm{HF}=\mathrm{A}(4801: 7199,:,:)$ :

CwDeltaEps. NelmLF $=$ zeros $(($ Period. N/2 -1$) * 100,1)$;

CwDeltaEps. NelmHF $=$ zeros $(($ Period . N/2*2/3-1)*100,1);

for $c=1$ : ceil $(\max (\max (|\mathrm{t} v|$. PosAscStrainPMeanStress $(:,:))) / \mathrm{cw})$

elm If $=\operatorname{LF}(3599 * 100 *(c-1)+1: 3599 * 100 * c)$;

elm If $=\operatorname{elmIf}($ eImIf<0);

$\operatorname{SUMLF}(\mathrm{c})=\operatorname{sum}(\mathrm{elm} I \mathrm{f})$;

$\operatorname{ANZLF}(\mathrm{c})=\mathrm{nnz}(\mathrm{elm} I \mathrm{f})$;

$\operatorname{MEANLF}(\mathrm{c})=\operatorname{SUMLF}(\mathrm{c}) / \operatorname{ANZLF}(\mathrm{c})$;

$\operatorname{STDLF}(\mathrm{c})=1 /(\operatorname{ANZLF}(\mathrm{c})-1) * \operatorname{sum}\left((\mathrm{e} \operatorname{mIf}-\operatorname{MEANLF}(\mathrm{c})) .{ }^{\wedge} 2\right)$;

$\operatorname{elmhf}=\operatorname{HF}(2399 * 100 *(\mathrm{c}-1)+1: 2399 * 100 * \mathrm{c})$;

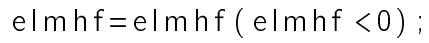

$\operatorname{SUMHF}(\mathrm{c})=\operatorname{sum}(\mathrm{elmhf})$;

$\operatorname{ANZHF}(\mathrm{c})=\mathrm{nnz}(\mathrm{eImhf})$;

$\operatorname{MEANHF}(\mathrm{c})=\operatorname{SUMHF}(\mathrm{c}) / \operatorname{ANZHF}(\mathrm{c})$;

$\operatorname{STDHF}(\mathrm{c})=1 /(\operatorname{ANZHF}(\mathrm{c})-1) * \operatorname{sum}\left((\operatorname{elmhf}-\operatorname{MEANHF}(\mathrm{c})) \cdot{ }^{\wedge} 2\right)$;

elmIf $=[$ elmIf,$z \operatorname{eros}(1,359900-\operatorname{length}($ elmIf $))]$;

CwDeltaEps. NelmLF $=[$ CwDeltaEps. NelmLF elmIf '] ;

$\operatorname{elmhf}=[\operatorname{elmhf}, \operatorname{zeros}(1,239900-$ length $(\operatorname{elmhf}))]$;

CwDeltaEps. NelmHF $=[$ CwDeltaEps. NelmHF elmhf'];

CwDeltaEps. NLF $(c, 1)=\operatorname{SUMLF}(\mathrm{c})$;

CwDeltaEps. NLF $(c, 2)=\operatorname{ANZLF}(\mathrm{c})$;

CwDeltaEps.NLF $(c, 3)=\operatorname{MeANLF}(\mathrm{c})$;

CwDeltaEps. NLF $(c, 4)=\operatorname{STDLF}(\mathrm{c})$;

CwDeltaEps. $\operatorname{NHF}(c, 1)=\operatorname{SUMHF}(\mathrm{c})$;

CwDeltaEps. $\operatorname{NHF}(c, 2)=\operatorname{ANZHF}(\mathrm{c})$;

CwDeltaEps.NHF $(c, 3)=\operatorname{MEANHF}(\mathrm{c})$;

CwDeltaEps. NHF $(c, 4)=\operatorname{STDHF}(\mathrm{c})$;

end

clear elmhf elmIf

clear A SUMLF ANZLF MEANLF STDLF SUMHF ANZHF MEANHF STDHF $c$ LF HF

$\mathrm{B}=\mathrm{C}$ w DeltaSig. $\mathrm{N}$;

$\mathrm{LF}=\mathrm{B}(1: 3599,:,:)$;

$\mathrm{HF}=\mathrm{B}(4801: 7199,:,:)$;

CwDeltaSig. NelmLF $=$ zeros $(($ Period $. N / 2-1) * 100,1)$;

CwDeltaSig. NelmHF $=$ zeros $(($ Period. N/2*2/3-1)*100,1);

for $c=1$ : ceil $(\max (\max (|\mathrm{t} v|$. PosAscStrainPMeanStress $(:,:))) / \mathrm{cw})$

elm If $=\operatorname{LF}(3599 * 100 *(c-1)+1: 3599 * 100 * c)$;

eIm If $=\operatorname{eImIf}($ elm If $>0)$;

$\operatorname{SUMLF}(\mathrm{c})=\operatorname{sum}(\mathrm{elm} I \mathrm{f})$;

$\operatorname{ANZLF}(\mathrm{c})=\mathrm{nnz}(\mathrm{elm} \mathrm{If})$;

$\operatorname{MEANLF}(\mathrm{c})=\operatorname{SUMLF}(\mathrm{c}) / \operatorname{ANZLF}(\mathrm{c})$;

$\operatorname{STDLF}(\mathrm{c})=1 /(\operatorname{ANZLF}(\mathrm{c})-1) * \operatorname{sum}((\operatorname{elm} \mathbf{I f}-\operatorname{MEANLF}(\mathrm{c})) . \wedge 2)$;

$\operatorname{elmhf}=\mathrm{HF}(2399 * 100 *(\mathrm{c}-1)+1: 2399 * 100 * \mathrm{c})$;

$\operatorname{elmhf}=\operatorname{elmhf}(\operatorname{elmhf}>0)$;

$\operatorname{SUMHF}(\mathrm{c})=\operatorname{sum}(\mathrm{elmhf})$;

$\operatorname{ANZHF}(\mathrm{c})=\mathrm{nnz}(\mathrm{elmhf})$;

$\operatorname{MEANHF}(\mathrm{c})=\operatorname{SUMHF}(\mathrm{c}) / \operatorname{ANZHF}(\mathrm{c})$;

$\operatorname{STDHF}(\mathrm{c})=1 /(\operatorname{ANZHF}(\mathrm{c})-1) * \operatorname{sum}((\operatorname{elmhf}-\operatorname{MEANHF}(\mathrm{c})) . \wedge 2)$;

elmIf $=[\operatorname{elmIf}, \operatorname{zeros}(1,359900-$ length $($ elmIf $))]$;

CwDeltaSig. NelmLF $=$ [CwDeltaSig. NelmLF elmIf'];

$\operatorname{elmhf}=[\operatorname{elmhf}, \operatorname{zeros}(1,239900-\operatorname{length}(\mathrm{elmhf}))]$; 


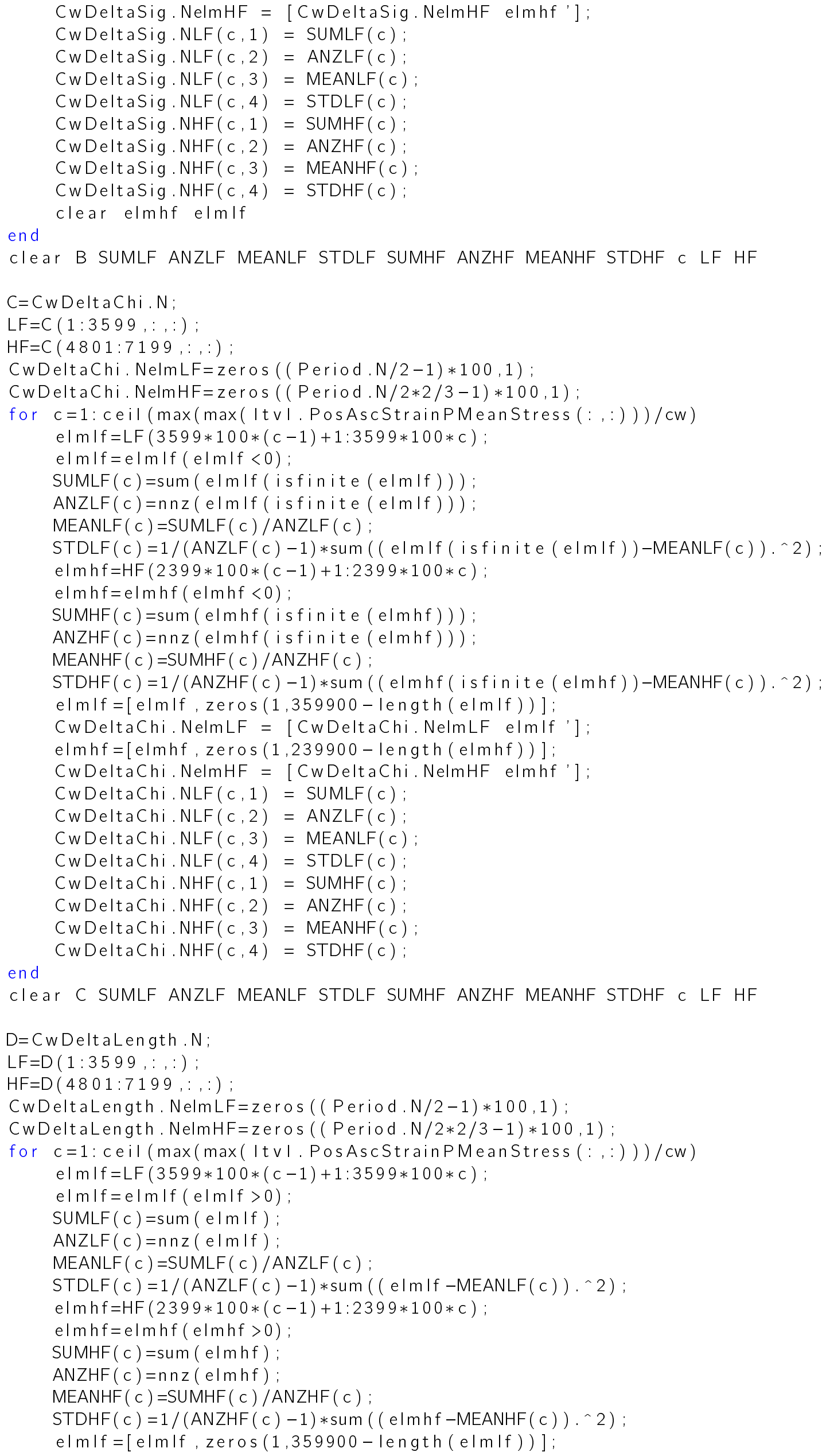


CwDeltaLength. NelmLF $=$ [CwDeltaLength. NelmLF elmIf ']; $\operatorname{elmhf}=[\operatorname{elmhf}, \operatorname{zeros}(1,239900-\operatorname{length}(\mathrm{elmhf}))]$;

CwDeltaLength. NelmHF $=[$ CwDeltaLength. NelmHF elmhf'];

CwDeltaLength. $\operatorname{NLF}(c, 1)=\operatorname{SUMLF}(c)$;

CwDeltaLength. NLF $(c, 2)=\operatorname{ANZLF}(c)$;

CwDeltaLength. NLF $(c, 3)=\operatorname{MEANLF}(\mathrm{c})$;

CwDeltaLength. $\operatorname{NLF}(c, 4)=\operatorname{STDLF}(\mathrm{c})$;

CwDeltaLength. NHF $(c, 1)=\operatorname{SUMHF}(c)$;

CwDeltaLength. NHF $(c, 2)=\operatorname{ANZHF}(c)$;

CwDeltaLength $\operatorname{NHF}(c, 3)=\operatorname{MEANHF}(\mathrm{c})$;

end

CwDeltaLength. NHF $(c, 4)=\operatorname{STDHF}(c)$;

clear D SUMLF ANZLF MEANLF STDLF SUMHF ANZHF MEANHF STDHF $c$ LF HF

PosAscPN

$\%$

CwDeltaEps.PN=zeros (Period. N-1,100, ceil $(\max (\max (|\mathrm{t} v|$.

PosAscStrainNMeanStress $(:,:))) / c w))$;

CwDeltaChi.PN=zeros (Period. $N-1,100$, ceil ( $\max (\max (|\mathrm{t} v|$.

PosAscStrainNMeanStress $(:,:))) / c w))$;

CwDeltaOmega. PN=zeros (Period. $N-1,100$, ceil $(\max (\max (\mid \mathrm{t}$ vI .

PosAscStrainNMeanStress $(:,:))) / c w))$;

CwDeltaLength. PN=zeros (Period. $N-1,100$, ceil $(\max (\max (|\mathrm{t} v|$.

PosAscStrainNMeanStress (: , : ) )) /cw) ) ;

PosAscPNChi=zeros (Period. Length / 4, Period. N-1);

PosAscPNStrain=zeros (Period. Length $/ 4$, Period . N-1);

PosAscPNMeanStress=zeros (Period. Length / 4, Period . N-1);

for $\mathrm{p}=1$ : Period. $\mathrm{N}-1$

$\% \quad \mathrm{I}=1$;

if $|t v|$. PosAscStrainNMeanStress $(1, p)<|t v|$. PosAscStrainPMeanStress $(1, p)$

for $i=1: 2: n n z(|t v|$. PosAscStrainNLength $(:, p))$

PosAscPNChi $(i, p)=(|t v|$. PosAscStrainNDelta $(i, p)+|t v|$.

PosAscStrainPDelta ( $i, p)) /($ ItvI. PosAscStrainNDeltaStress ( $i$

$, p)+|t v|$. PosAscStrainPDeltaStress (i, p) )*10;

PosAscPNStrain $(i, p)=|t v|$. PosAscStrainNDelta $(i, p)+|t v|$. PosAscStrainPDelta $(i, p)$;

PosAscPNMeanStress $(i, p)=($ Itv I. PosAscStrainNMeanStress $(i, p)+$ ItvI. PosAscStrainPMeanStress (i,p)) / 2;

PosAscPNLength $(i, p)=(|t v|$. PosAscStrainNLength $(i, p)+|t v|$.

PosAscStrainPLength (i,p));

if isfinite (PosAscPNChi $(i, p))==0$

PosAscPNChi $(i, p)=0$;

elseif PosAscPNChi(i,p) > 100000

$\operatorname{PosAscPNChi}(i, p)=0$;

end

if $(i+1)<=n n z(|t v|$. PosAscStrainPLength $(:, p))$

PosAscPNChi $(i+1, p)=(\mid$ t $v \mid$. PosAscStrainNDelta $(i+1, p)+\mid$ t $v \mid$. PosAscStrainPDelta $(i, p)) /(|t v|$.

PosAscStrainNDeltaStress $(i+1, p)+|t v|$

PosAscStrainPDeltaStress ( $\mathrm{i}, \mathrm{p})) * 10$;

PosAscPNStrain $(i+1, p)=|t v|$. PosAscStrainNDelta $(i+1, p)+$ ItvI. PosAscStrainPDelta $(i, p)$;

PosAscPNMeanStress $(i+1, p)=(\mid$ t $v \mid$. PosAscStrainNMeanStress $(i+1, p)+|t v|$. PosAscStrainPMeanStress $(i, p)) / 2$;

PosAscPNLength $(i+1, p)=(\mid$ t $v \mid$. PosAscStrainNLength $(i+1, p)+$ $\mid$ tv $\mid$. PosAscStrainPLength $(i, p))$;

if isfinite (PosAscPNChi $(i+1, p))==0$

PosAscPNChi $(i+1, p)=0$;

elseif PosAscPNChi $(i+1, p)>100000$

$\operatorname{PosAscPNChi}(i+1, p)=0$;

end

end

end

else 


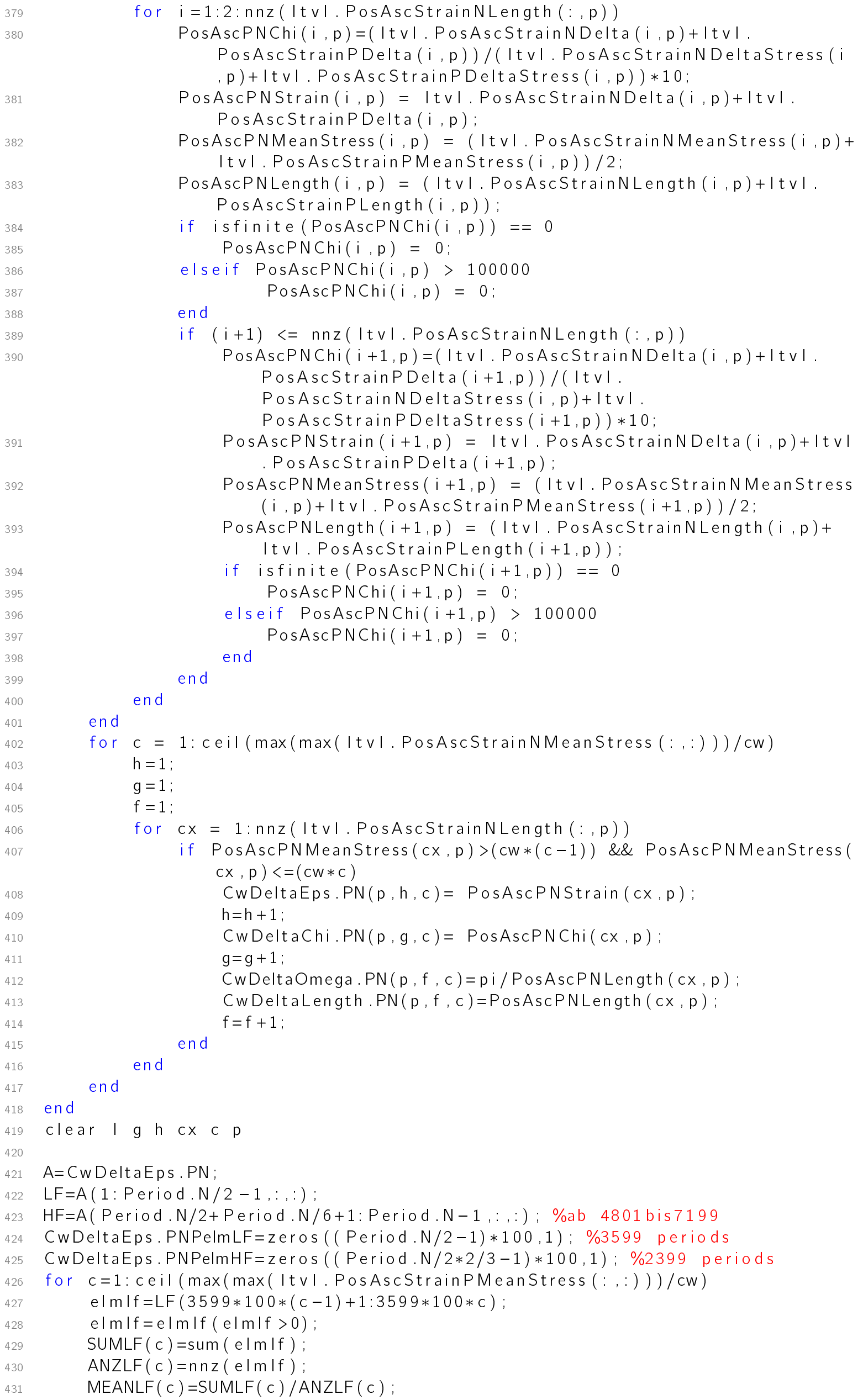


$\operatorname{STDLF}(\mathrm{c})=1 /(\operatorname{ANZLF}(\mathrm{c})-1) * \operatorname{sum}((\operatorname{elm} \mathbf{I f}-\operatorname{MEANLF}(\mathrm{c})) . \wedge 2)$; $\operatorname{elmhf}=\operatorname{HF}(2399 * 100 *(\mathrm{c}-1)+1: 2399 * 100 * \mathrm{c})$;

$\operatorname{elmhf}=\operatorname{eImhf}(\operatorname{elmhf}>0)$;

$\operatorname{SUMHF}(\mathrm{c})=\operatorname{sum}(\mathrm{eImhf})$;

$\operatorname{ANZHF}(\mathrm{c})=\mathrm{nnz}(\mathrm{eImhf})$;

$\operatorname{MEANHF}(\mathrm{c})=\operatorname{SUMHF}(\mathrm{c}) / \operatorname{ANZHF}(\mathrm{c})$;

$\operatorname{STDHF}(\mathrm{c})=1 /(\operatorname{ANZHF}(\mathrm{c})-1) * \operatorname{sum}\left((\operatorname{elmhf}-\operatorname{MEANHF}(\mathrm{c})) .{ }^{\wedge} 2\right)$;

elmIf $=[$ elmIf,$z \operatorname{eros}(1,359900-$ length $($ elmIf $))]$;

CwDeltaEps. PNPelmLF $=$ [CwDeltaEps. PNPelmLF elmIf '] ;

$\operatorname{elmhf}=[\operatorname{elmhf}, \operatorname{zeros}(1,239900-\operatorname{length}(\mathrm{elmhf}))]$;

CwDeltaEps. PNPelmHF = [CwDeltaEps. PNPelmHF elmhf'];

CwDeltaEps.PNPLF $(c, 1)=\operatorname{SUMLF}(\mathrm{c})$;

CwDeltaEps.PNPLF $(c, 2)=\operatorname{ANZLF}(c)$;

CwDeltaEps.PNPLF $(c, 3)=\operatorname{MEANLF}(\mathrm{c})$;

CwDeltaEps.PNPLF $(c, 4)=\operatorname{STDLF}(\mathrm{c})$;

CwDeltaEps.PNPHF $(c, 1)=\operatorname{SUMHF}(\mathrm{c})$;

CwDeltaEps.PNPHF $(c, 2)=\operatorname{ANZHF}(\mathrm{c})$;

CwDeltaEps.PNPHF $(c, 3)=\operatorname{MEANHF}(c)$;

CwDeltaEps.PNPHF $(c, 4)=\operatorname{STDHF}(c)$;

end

clear elmhf elmIf

clear SUMLF ANZLF MEANLF STDLF SUMHF ANZHF MEANHF STDHF c

CwDeltaEps. PNNelmLF $=$ zeros $(($ Period . N/2 - 1) $* 100,1)$;

CwDeltaEps.PNNelmHF $=$ zeros ( ( Period. N/2*2/3-1)*100,1);

for $c=1$ : ceil $(\max (\max (|\mathrm{t} v|$. PosAscStrainPMeanStress $(:,:))) / \mathrm{cw})$

$\mathrm{elmIf}=\operatorname{LF}(3599 * 100 *(\mathrm{c}-1)+1: 3599 * 100 * \mathrm{c})$;

eImIf $=\operatorname{eImIf}(\operatorname{eImIf<0);~}$

$\operatorname{SUMLF}(\mathrm{c})=\operatorname{sum}(\mathrm{elm} I \mathrm{f})$;

$\operatorname{ANZLF}(\mathrm{c})=\mathrm{nnz}(\mathrm{elmIf})$;

$\operatorname{MEANLF}(\mathrm{c})=\operatorname{SUMLF}(\mathrm{c}) / \operatorname{ANZLF}(\mathrm{c})$;

$\operatorname{STDLF}(\mathrm{c})=1 /(\operatorname{ANZLF}(\mathrm{c})-1) * \operatorname{sum}((\mathrm{elm} \operatorname{lf}-\operatorname{MEANLF}(\mathrm{c})) . \wedge 2)$;

$\operatorname{elmhf}=\mathrm{HF}(2399 * 100 *(\mathrm{c}-1)+1: 2399 * 100 * \mathrm{c})$;

elmhf $=\operatorname{elmhf}(\operatorname{elmhf}<0)$;

$\operatorname{SUMHF}(\mathrm{c})=\operatorname{sum}(\mathrm{elmhf})$;

$\operatorname{ANZHF}(\mathrm{c})=\mathrm{nnz}(\mathrm{elmhf})$;

$\operatorname{MEANHF}(\mathrm{c})=\operatorname{SUMHF}(\mathrm{c}) / \operatorname{ANZHF}(\mathrm{c})$;

$\operatorname{STDHF}(\mathrm{c})=1 /(\operatorname{ANZHF}(\mathrm{c})-1) * \operatorname{sum}((\operatorname{elmhf}-\operatorname{MEANHF}(\mathrm{c})) . \wedge 2)$;

elm If $=[$ elmIf, $\operatorname{zeros}(1,359900-$ length $($ elmIf $))]$;

CwDeltaEps.PNNelmLF = [CwDeltaEps. PNNelmLF elmIf '] ;

$\operatorname{elmhf}=[\operatorname{elmhf}, \operatorname{zeros}(1,239900-$ length $(\operatorname{elmhf}))]$;

CwDeltaEps.PNNelmHF = [CwDeltaEps.PNNelmHF elmhf'];

CwDeltaEps.PNNLF $(c, 1)=\operatorname{SUMLF}(\mathrm{c})$;

CwDeltaEps.PNNLF $(c, 2)=\operatorname{ANZLF}(c)$;

CwDeltaEps.PNNLF $(c, 3)=\operatorname{MEANLF}(\mathrm{c})$;

CwDeltaEps.PNNLF $(c, 4)=\operatorname{STDLF}(\mathrm{c})$;

CwDeltaEps.PNNHF $(c, 1)=\operatorname{SUMHF}(c)$;

CwDeltaEps.PNNHF $(c, 2)=\operatorname{ANZHF}(c)$;

CwDeltaEps.PNNHF $(c, 3)=\operatorname{MEANHF}(\mathrm{c})$;

CwDeltaEps.PNNHF $(c, 4)=\operatorname{STDHF}(\mathrm{c})$;

end

clear elmhf elmIf

clear A SUMLF ANZLF MEANLF STDLF SUMHF ANZHF MEANHF STDHF C LF HF

$\mathrm{B}=\mathrm{CwDelta}$ Chi .PN;

$\mathrm{LF}=\mathrm{B}(1: 3599,:,:)$;

$\mathrm{HF}=\mathrm{B}(4801: 7199,:,:)$;

CwDeltaChi. PNelmLF $=$ zeros $(($ Period $. N / 2-1) * 100,1)$;

CwDeltaChi.PNelmHF $=$ zeros ( (Period. N/2*2/3-1)*100,1);

for $c=1$ : ceil $(\max (\max (|\mathrm{t} v|$. PosAscStrainPMeanStress $(:,:))) / \mathrm{cw})$

elm If $=\operatorname{LF}(3599 * 100 *(\mathrm{c}-1)+1: 3599 * 100 * \mathrm{c})$;

elmIf $=\operatorname{eImIf}(\operatorname{eImIf} \sim=0)$;

$\operatorname{SUMLF}(\mathrm{c})=\operatorname{sum}(\mathrm{elm} I \mathrm{f})$;

$\operatorname{ANZLF}(\mathrm{c})=\mathrm{nnz}(\mathrm{eImIf})$;

$\operatorname{MEANLF}(\mathrm{c})=\operatorname{SUMLF}(\mathrm{c}) / \operatorname{ANZLF}(\mathrm{c})$;

$\operatorname{STDLF}(\mathrm{c})=1 /(\operatorname{ANZLF}(\mathrm{c})-1) * \operatorname{sum}\left((\operatorname{elm} \mathrm{If}-\operatorname{MEANLF}(\mathrm{c})) .{ }^{\wedge} 2\right)$; 


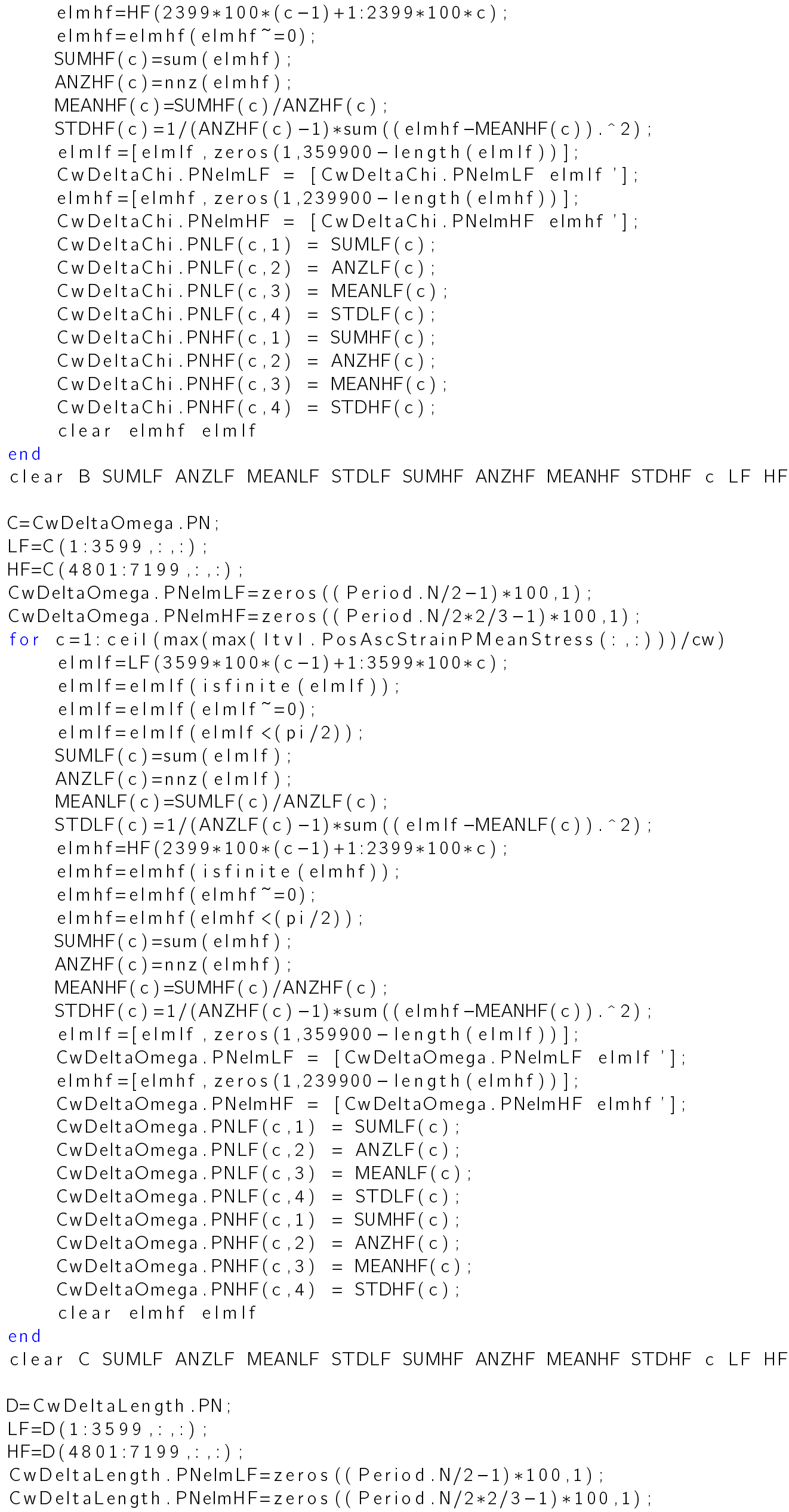




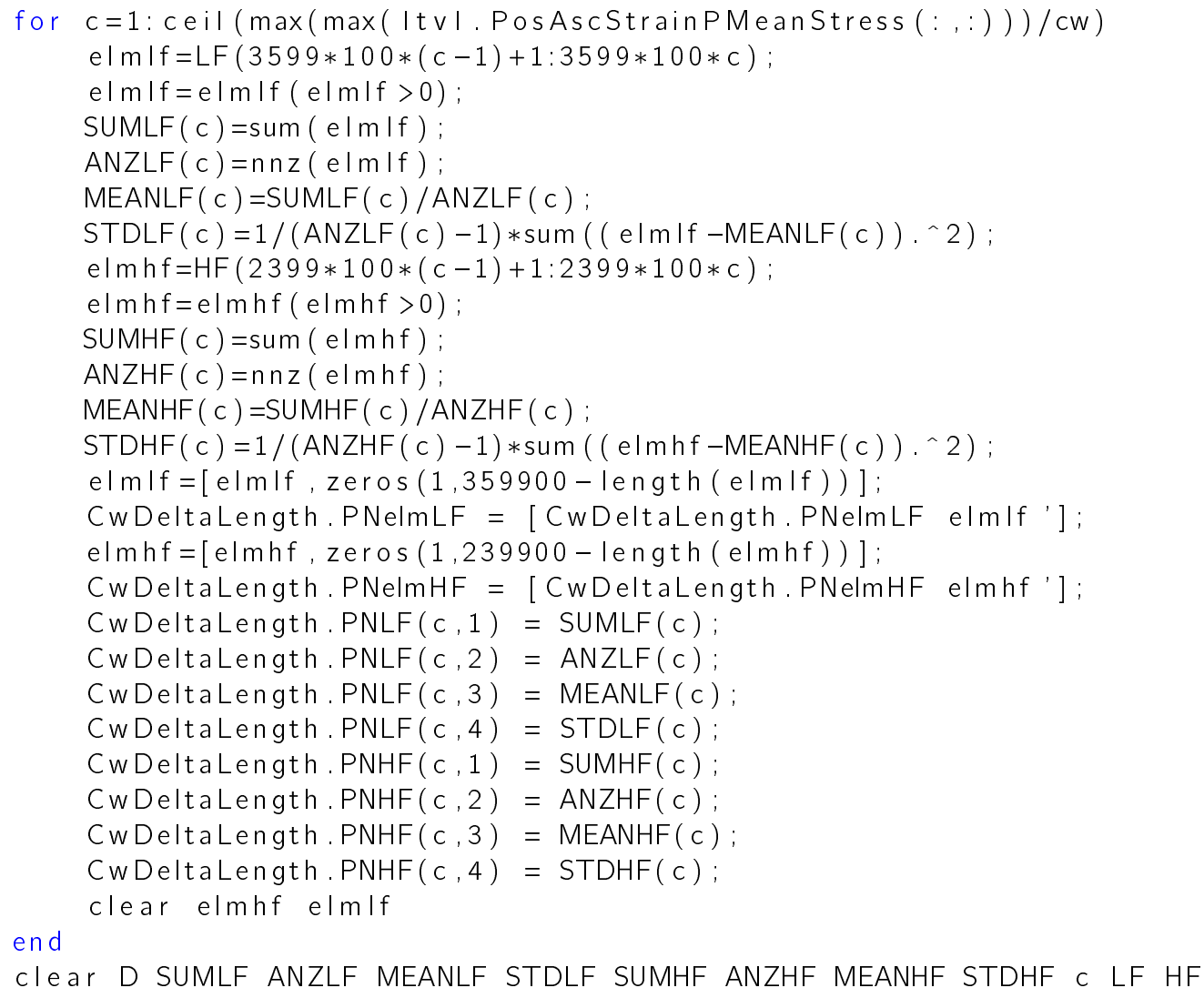

\section{E.4 Extraction of Strain Interval Distributions}

The following code is based on the subdivision of interval data, as generated by the code given in section E.3 (Subdivision to Stress-Windows). It derives the distribution of interval strain, which is presented in this thesis in terms of the distributions based on the first $2 / 3$ of each period.

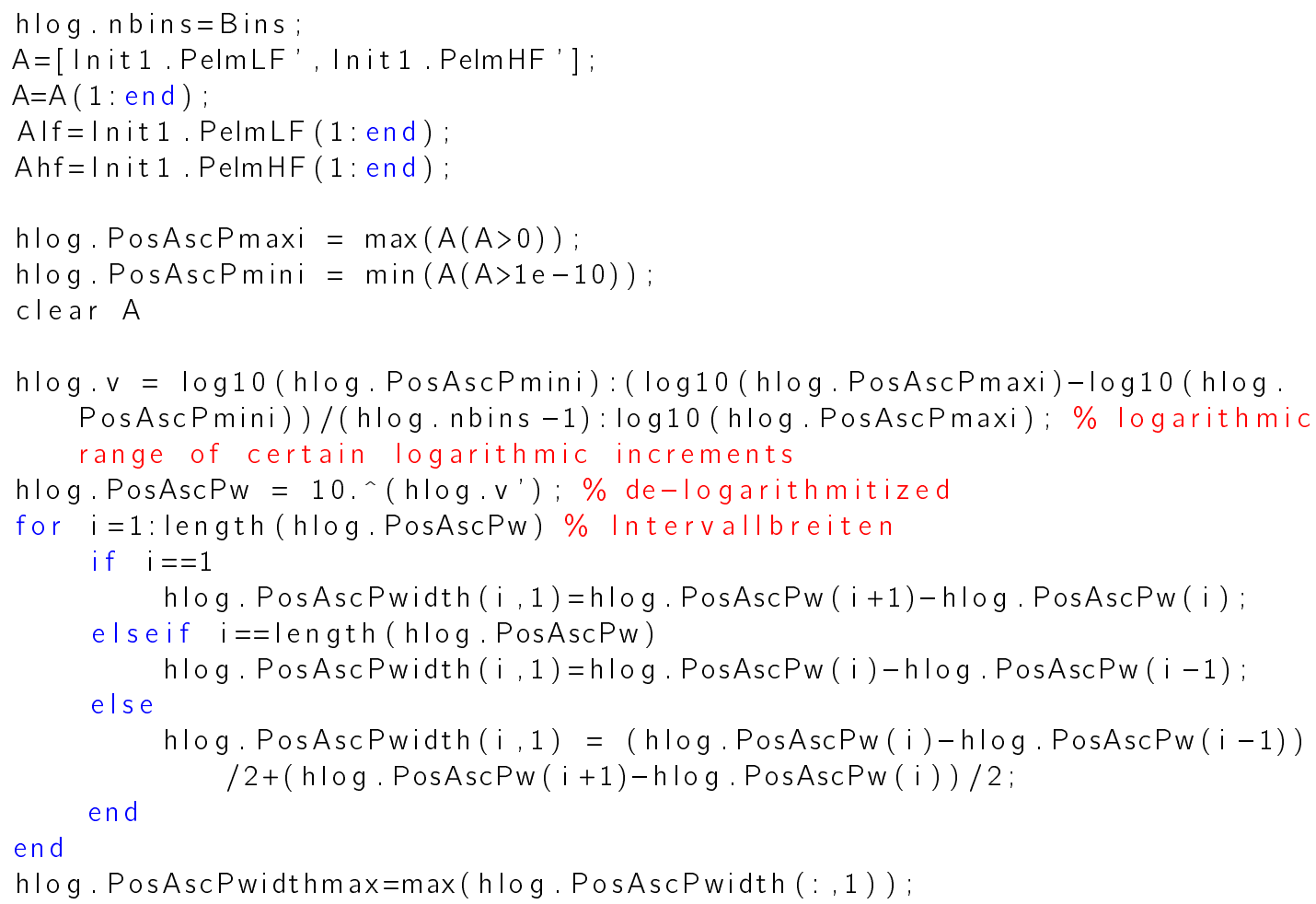




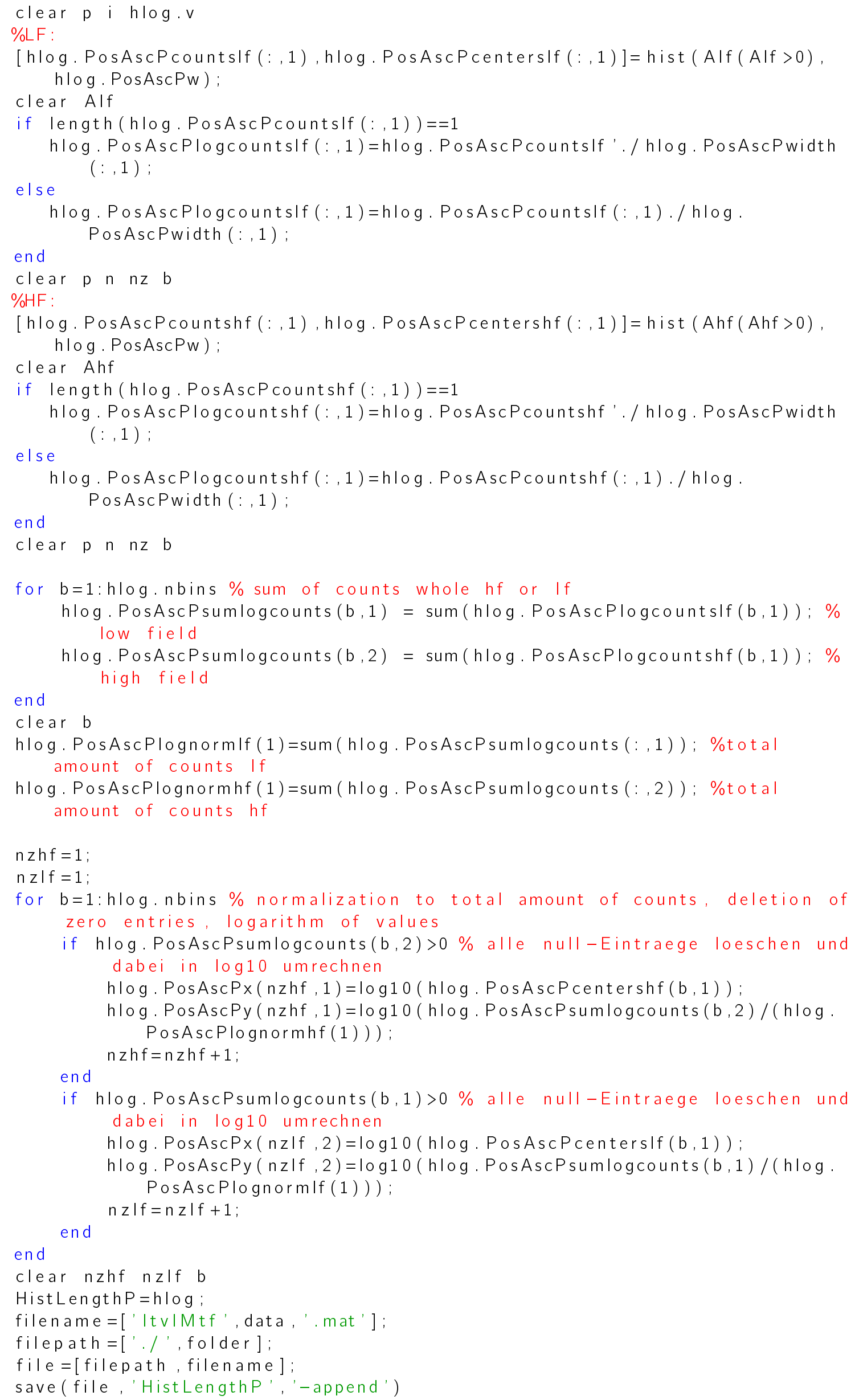




\section{Appendix F}

\section{Influence of Data Point Density}

The interval data is analyzed regarding dependencies on parameters like the stress amplitude $\sigma_{0}$, the strain rate $\dot{\varepsilon}$, and the data point density $\rho_{D P}$. Upon these quantities, the data point density is not defined by intrinsic material response or the excitation itself, but is due to the subdivision of the data into stress-windows, similar to a binning. It can be deduced by equation $\mathrm{F} .1$ and is defined as the number of data points within a certain stress window $\Delta \sigma_{c}=\sigma_{f}-\sigma_{i}$ of the positive ascending part of the sine. Due to the sinusoidal form of the stress excitation $\sigma_{c}=\sigma_{0} \cdot \sin (\omega T)$, the number of data points per stress window, which is equal to the duration $\Delta t_{c}$ in $\mathrm{ms}$, increases with the value of intra-periodic stress $\sigma_{c}$. Thus, the data point density $\rho_{d p}$ increases with intra-periodic stress $\sigma_{c}$ as well.

$$
\rho_{d p}=\frac{\Delta t_{c}}{t_{t o t}}=(2 \pi f)^{-1} \cdot\left[\arcsin \left(\frac{\sigma_{f}}{\sigma_{0}}\right)-\arcsin \left(\frac{\sigma_{i}}{\sigma_{0}}\right)\right] / t_{t o t}
$$

If the arcus-sine is written in a series expansion and terms which are of squared or higher order are neglected, the data point density can be written as:

$$
\rho_{d p} \approx(2 \pi f)^{-1} \cdot \frac{\Delta \sigma_{c}}{\sigma_{0}}
$$

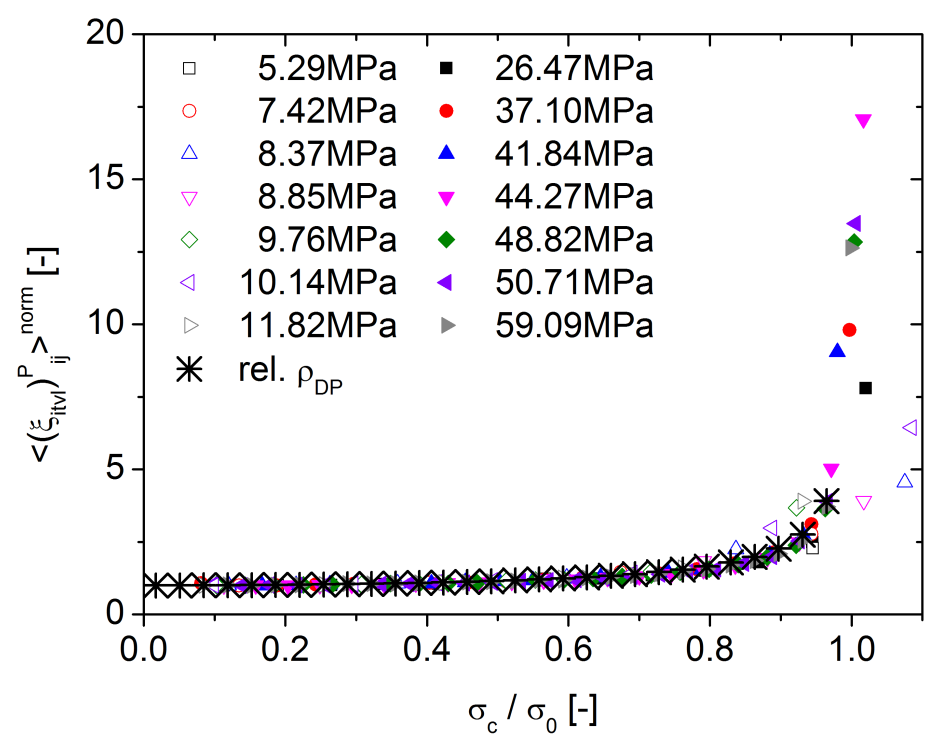

Figure F.1 Normalized averaged interval compliance $\left\langle\left(\xi_{i t v l}\right)_{i j}^{P}\right\rangle^{\text {norm }}$ in comparison the the normalized data point density $\rho_{D P}$ plotted against the normalized intra-periodic stress $\sigma_{c} / \sigma_{0}$, as exemplification on positive strain intervals.

The interval strain $\Delta \varepsilon_{i t v l}$ and the interval duration $\Delta t_{i t v l}$ do not show a dependence on the 
data point density, but are rather dominated by the strain rate: In contrast, the influence of $\rho_{D P}$ on the interval apparent compliance $\xi_{i t v l}$, the interval width $\Delta \sigma_{i t v l}$, and the number of intervals $N_{i t v l}$ is dominant. This is shown in fig. F.1 to fig. F.3 and becomes evident by the conformity in the intra-periodic evolution. Thus, these three interval quantities are excluded from the discussion on a relation to avalanche behavior.

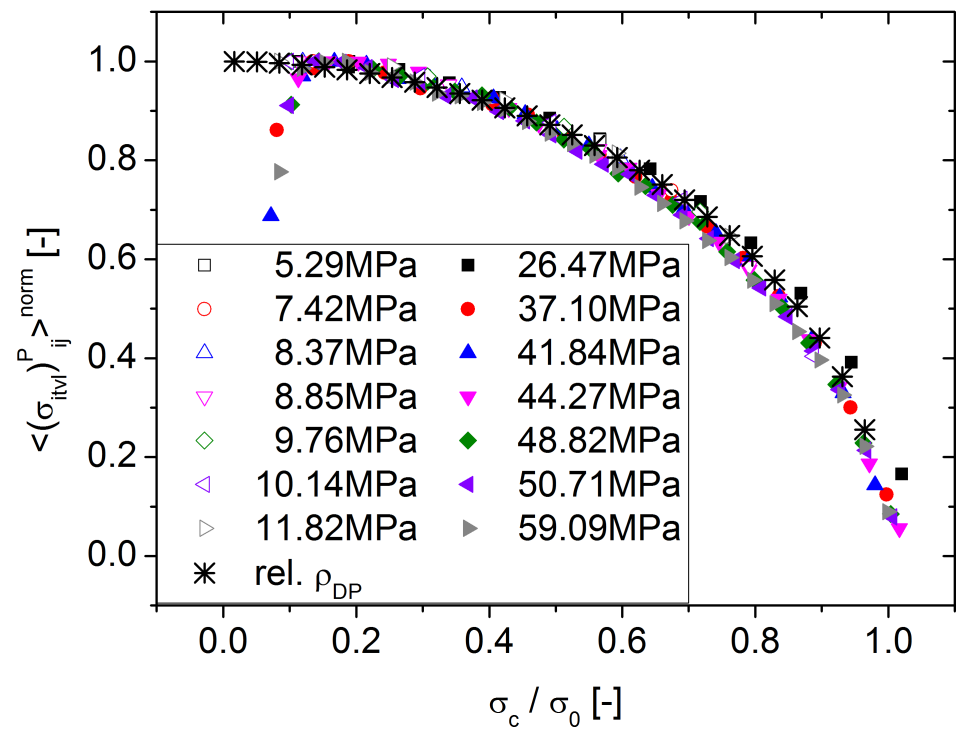

Figure F.2 Normalized averaged interval width $\left\langle\left(\Delta \sigma_{i t v l}\right)_{i j}^{P}\right\rangle^{\text {norm }}$ in comparison the the normalized data point density $\rho_{D P}$ plotted against the normalized intra-periodic stress $\sigma_{c} / \sigma_{0}$, as exemplification on positive strain intervals.

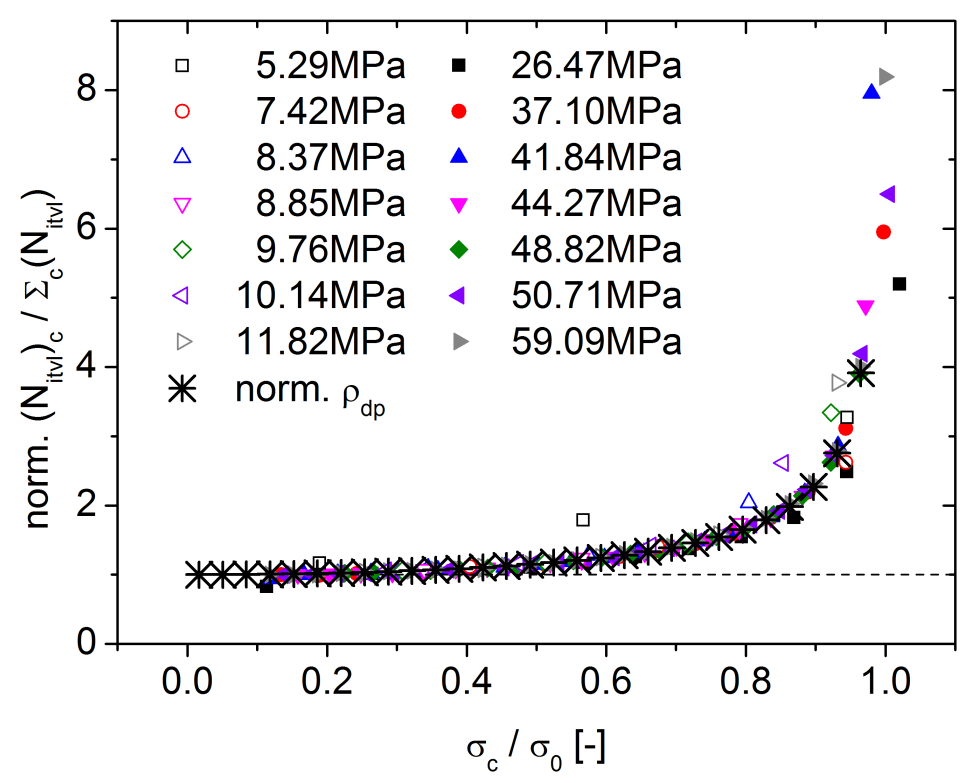

Figure F.3 Normalized umber of intervals $\left(N_{i t v l}\right)_{c} / \Sigma_{c}\left(N_{i t v l}\right)_{c}$ in comparison the the normalized data point density $\rho_{D P}$ plotted against the normalized intra-periodic stress $\sigma_{c} / \sigma_{0}$. 


\section{Appendix G}

\section{Distributions on ideal Sinusoidal Data}

In order to learn about the influence of the global sinusoidal form of the data within each period, the excitation signal which is of ideal sinusoidal form is analyzed according to its increments and intervals. As this ideal sinusoidal data does not inherit any interval-like behavior, the interval-definition is based on the strain evaluation. Thus, the evaluation of the data in this manner generates an artificial distribution, which will give an estimate on the influence of the sinusoidal form itself, but does not hold any information regarding the physics of microstructural processes.

The limit of resolution in time is $1 \mathrm{~ms}$, the limit of stress-resolution is $0.01 \mathrm{MPa}$. The data resolution is identical for all evaluated sine waves, while the absolute amplitude $\sigma_{0}$ of the sine wave is changed in a range from 700 to 7850 times the resolution limit.

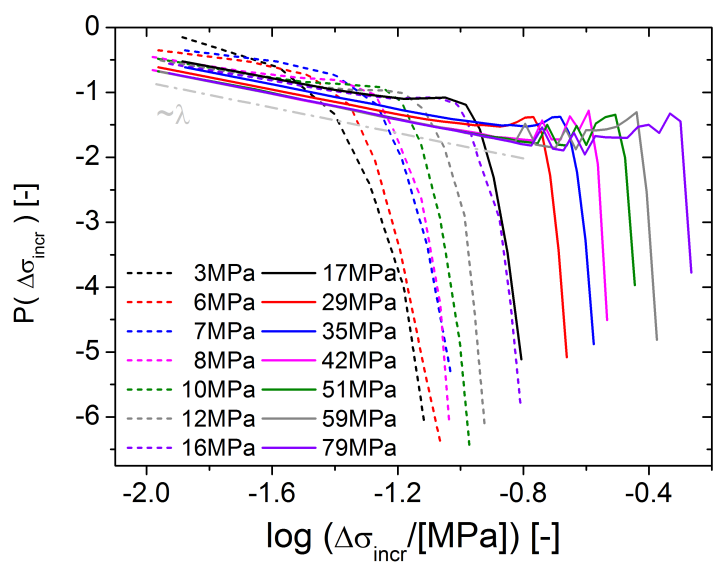

(a)

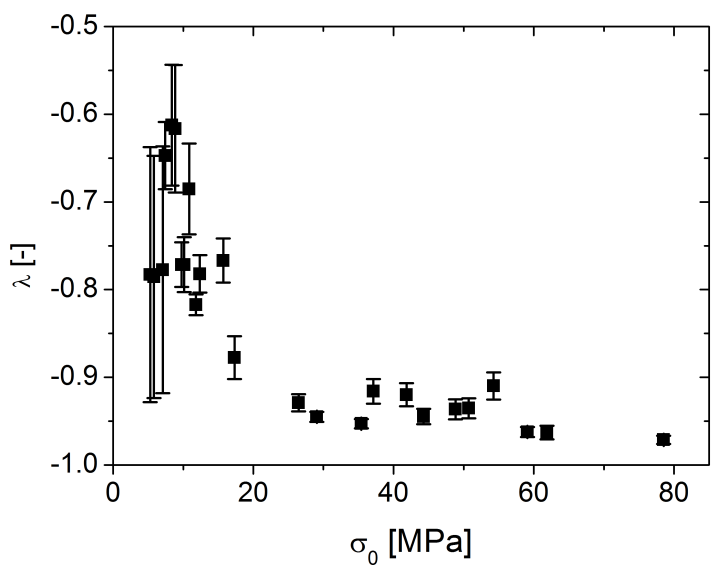

(b)

Figure G.1 (a) Distribution of increments in sinusoidal data for the full positive ascending part of the sine, based on $\Delta \sigma_{\text {incr }}$ for various stress amplitudes $\sigma_{0}$. (b) Exponent $\lambda$ extracted from distributions shown in (a) plotted against stress amplitude $\sigma_{0}$. 
In figure G.1a the distribution of incremental sinusoidal data for the first quarter of a sine wave (positive ascending part) is plotted both for initial low field (dashed lines) and high field (continuous lines). It is clearly visible that an analysis of the full first quarter of the sine yields a power-law distribution for small values of $\Delta \sigma_{i n c r}$. Moreover it becomes evident that with increasing amplitude of the sine a maximum at higher values of $\Delta \sigma_{i n c r}$ evolves in the distribution. To extract the exponent leading to the powerlaw in double-logarithmic representation, the data is linearly fitted in the range of the power-law behavior. The evolution of the exponent is shown in figure G.1b. It shows clearly that the slope of the fit approaches the value -1 with increasing sine amplitude $\sigma_{0}$, which is coming along with an increasing range of linearity in the double-logarithmic representation.

In figure G.2 distributions are shown that do only include incremental data up to $2 / 3$ of the full amplitude, which reduces the evaluated data to one half of the number of increments which is used for the evaluation presented in fig. G.1a. This shows clearly, that the power-law behavior evolves from those increments at the maximum part of the sine wave, where the slope of the sine ceases towards zero. Only for sinusoidal data with comparatively small stress amplitudes, the slope in the early regime of the distributions is similar to the linear regions in fig. G.1a, but the broad range of linearity in the log-log-representation is missing here. Thus, by limiting the contributing data to the initial 2/3 of the positive ascending part of the sine, the generation of power-law behavior by the sinusoidal form itself is suppressed.

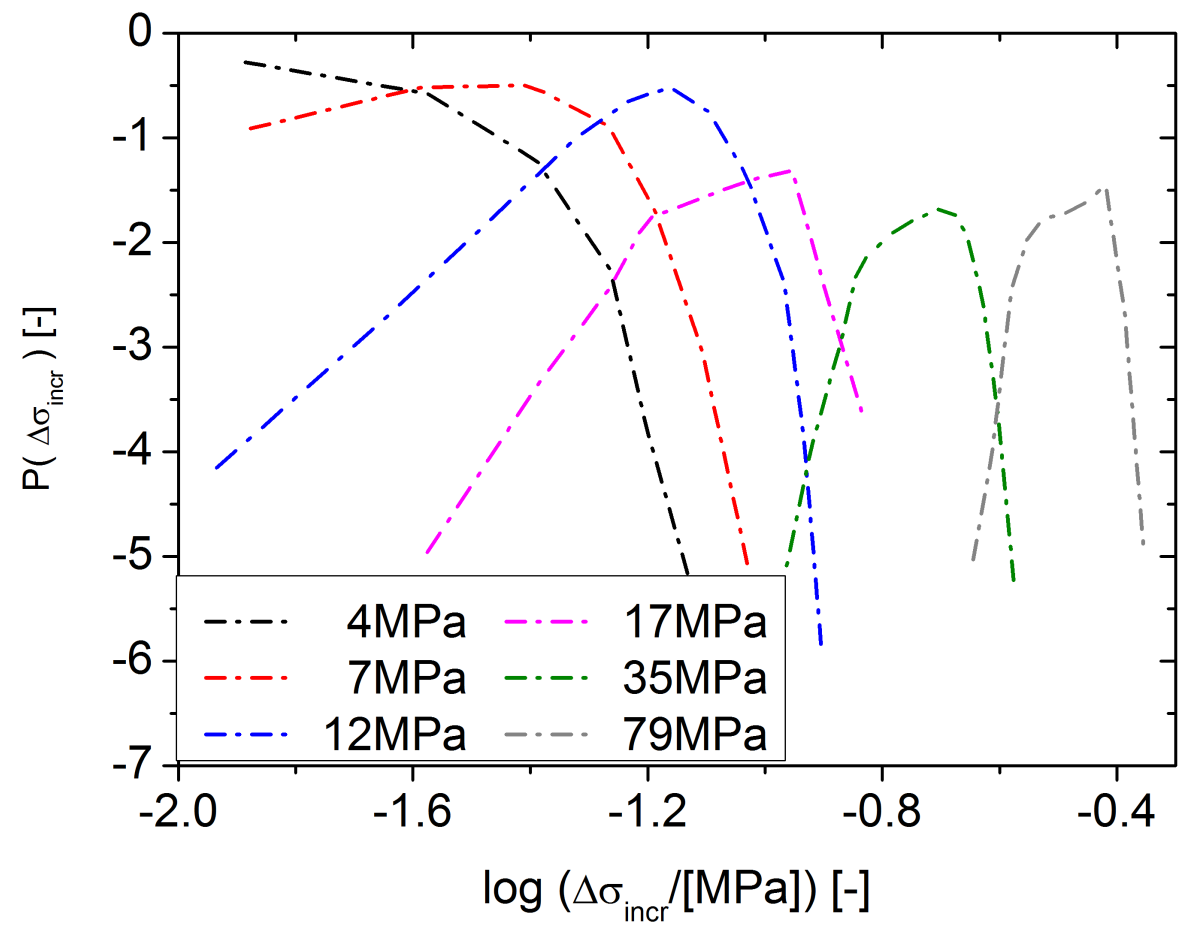

Figure G.2 Distribution of increments in sinusoidal data for the initial $2 / 3$ of the positive ascending part of the sine, based on $\Delta \sigma_{i n c r}$ occurring within the first two thirds of the stress amplitude (i.e. between stresses from 0 to $2 / 3 \cdot \sigma_{0}$ ) for various stress amplitudes $\sigma_{0}$.

As the strain data is evaluated interval-wise, it is reasonable to partition the sinusoidal data into intervals to check for the influence of the routine on the form of the distribution. To do so, stress data is evaluated with the interval partitioning gained by the evaluation of corresponding strain data. To give an example, distributions for interval partitioning according to positive intervals in strain are shown in fig. G.3 for different stress amplitudes.

While the distribution shifts towards higher values for the averaged stress intervals with increasing stress amplitude $\sigma_{0}$, the distribution becomes roughly linear due to the interval- 
wise analysis. The slope in the double-logarithmic plots is about -2.50 , and seems to be stable for a broad range of stress amplitudes. The range within the histogram is limited to roughly one order of magnitude. A power-law behavior exhibiting slopes comparable to values observed in fig. G.1a is not arising in case of interval-based analysis of ideal sinusoidal data originating from the initial $2 / 3$ of the positive ascending part of the sine.

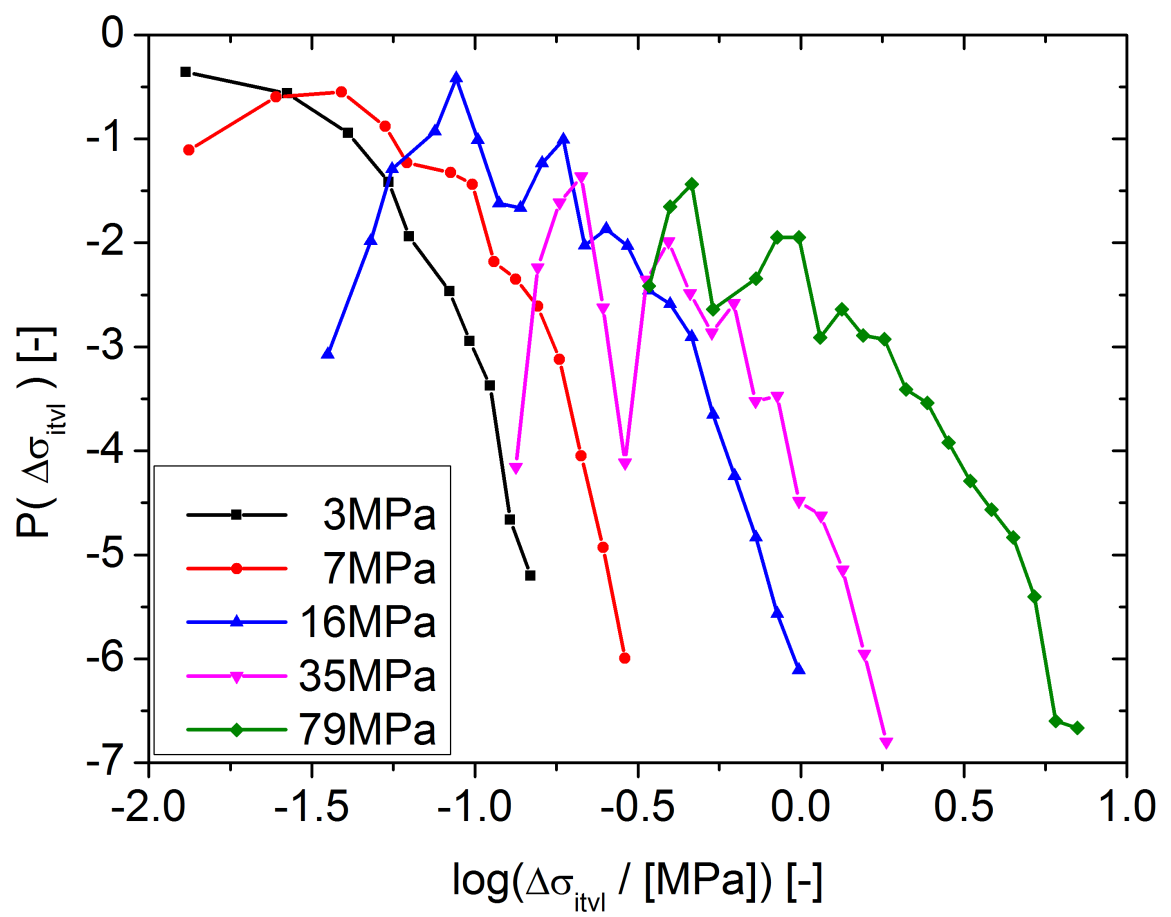

Figure G.3 Distribution of intervals in sinusoidal data, based on $\Delta \sigma_{i t v l}$ occurring within the first two thirds of the stress amplitude (i.e. between stresses from 0 to $2 / 3 \cdot \sigma_{0}$ ) for various stress amplitudes $\sigma_{0}$. 


\section{List of Figures}

2.1 (a) Volume $V$ plotted against temperature $T$. (b) Specific heat $C_{P}$ plotted against temperature $T$. Both giving a schematic example for the first order phase transition from the liquid to the crystalline state, and the transition from the liquid, via the supercooled liquid, to the glassy state, cf. [38]. . . . 4

2.2 Plot of the glass transition temperature $T_{g}$ against frequency $f$ connected to the experimental timescale for poly-3:3-biscloromethyloacyclobutane. Experimental techniques are dielectric spectroscopy (dielectric loss, $\mathrm{f}=10^{3} \mathrm{~Hz}$ ), mechanical vibration (mechanical loss, $f=89 \mathrm{~Hz}$ ), slow tensile deformation $(\mathrm{f}=3 \mathrm{~Hz})$, and dilatometry $\left(\mathrm{f}=10^{-2} \mathrm{~Hz}\right)[38] . \ldots \ldots \ldots$

2.3 Angell-plots of viscosity $\eta$ against normalized, inverse temperature $T_{g} / T$ for different amorphous systems. (a) According to [4]. (b) According to [67].] . 6

2.4 Scheme of the potential energy landscape [27]. . . . . . . . . . . . 8

2.5 Schematic description of differences in the potential energy landscape for fragile and strong materials [27]. . . . . . . . . . . . . . . . . . . . . . . . . . 9

2.6 (a) Scheme of the potential energy landscape focusing on $\alpha$ - and $\beta$-relaxations. Changes between adjacent basins correspond to $\beta$-processes, while the $\alpha$ process is referred to as a transition from one metabasin to another. (b) Scheme of the peak relaxation frequency against inverse temperature for both primary and secondary relaxation processes [133]. . . . . . . . . . . . . 10

2.7 Schematic plot of dielectric loss versus frequency for glass-formers obtained by broadband spectroscopy for temperatures $T_{1}$ and $T_{2}[85]$. . . . . . . . . 10

2.8 (a) Böhmer-plot of fragility $m$ against KWW-exponent $\beta_{K W W}$ for different classes of materials [14]. (b) Intrinsically exponential or non-exponential relaxations due to a heterogeneous or homogeneous explanation of the overall non-exponential form of the distribution of relaxations [111]. . . . . . . . . . 11

2.9 A schematic plot of the back and forth motion of string-like, cooperative $\beta$-processes (depicted in (a)) evolving in diffusion of single atoms (colored red in (b)) [159]. . . . . . . . . . . . . . . . . . . . . . . . . . . . 12

2.10 Plot of mechanical loss G" versus temperature $T$ for glass-formers obtained by dynamical mechanical analysis at constant frequency [119]. . . . . . . . . . 14

2.11 Force response to simple shear on the left and non-affine displacement field for the same state on the right based on numerical analysis [89]. . . . . . . . 15

2.12 Potential energy $U$ as a function of strain $\epsilon$ under quasi-static shear based on numerical analysis [88]. . . . . . . . . . . . . . . . . . . . . 15

2.13 Instantaneous velocity field of a growing shear transformation zone, triggering the flip of another zone in its vicinity based on MD simulations [82]. . . . . . 16

2.14 Spatial distribution of strain (a) in $y$-z- and (b) $x$-z-plane for an STZ, with particles colored corresponding to their shear strain (cf. colorscale in fig. 2.15) based on experiments on colloidal systems [122]. . . . . . . . . . 16 
2.15 Evolution of strain with experimental time after $20 \mathrm{~min}(\mathbf{A}), 30 \mathrm{~min}(\mathbf{B})$, and 50 min (C) with arrows indicating $\mid S T Z \$$ for $x$-y-frames of $5 \mu \mathrm{m}$ thickness in z-direction. $\mathbf{D}$ to $\mathbf{F}$ for same experimental times, but including highly strained particles for $x-y$-frames of $16 \mu \mathrm{m}$ thickness in z-direction, based on experiments on colloidal systems [122].

2.16 Stress-strain-curves measured under compressive mechanical load up to six different total strains on a metallic glass [52]. . . . . . . . . . . . . 18

2.17 Mechanical deformation map depicting deformation mechanisms in metallic glasses depending on normalized temperature $T / T_{g}$, normalized stress $\tau / \mu$,

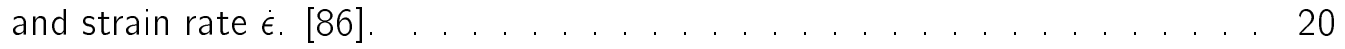

2.18 Influence of temperature $T$ and stress on the damping controlled delay-time of $\mathrm{Pd}_{77.5} \mathrm{Cu}_{6.0} \mathrm{Si}_{16.5}$ determined by mechanical creep measurements [126]. . . 21

2.19 (a) Mechanical loss $E$ " as a function of temperature $T$, and (b) mechanical loss $E$ as a function of temperature $T$ and strain amplitude $\varepsilon_{A}$, both based on MD-simulations [157].

2.20 Normalized fragility index $\operatorname{Norm}(m)=m_{\text {measured }} / m_{\varepsilon_{A}, \min }$ as a function of strain amplitude $\varepsilon_{A}$ deduced from MD-simulations [157].

2.21 Scheme of the change of the potential energy landscape due to applied shear stress [59].

2.22 Scheme of nonlinear response as a third-order polynomial (solid line) deviating from the linear behavior (dashed line) for an exciting electric field $E$ and polarization response $P[113]$

2.23 Dielectric loss $\varepsilon^{\prime \prime}(\nu)$ (symbols) aqainst frequency $\nu$ for glycerol at $T=213 \mathrm{~K}$ at a low-field amplitude of $E_{0}=14 \mathrm{kV} \mathrm{cm}^{-1}$ (lower data) and a high-field amplitude of $E_{0}=283 \mathrm{kV} \mathrm{cm}^{-1}$ (upper data). The line is based on assumptions due to the box-model [148].

2.24 Scheme of independent slow modes, weakly coupled to the phonon bath and

\begin{tabular}{|l}
\hline thus exhibiting slow relaxation of the energy absorbed from an external field. \\
\hline \hline Thus, an increase in effective temperature and a shift in the high-frequency
\end{tabular} flank of the dielectric loss spectrum are expected [57].

2.25 Field amplitude dependence of relative change in dielectric loss $\Delta \ln \varepsilon$ " vs. frequency. (a) for a structural glass-former [148], (b) for a mono-hydroxy alcohol [58]. 18 
3.2 Scheme of the sample mount in single cantilever mode in the DMA 8000 [40]. The three short arrows indicate the sample thickness, while the long arrow depicts the sample length. $\ldots \ldots \ldots \ldots$

3.3 Scheme of the LAOS excitation protocol. . . . . . . . . . . . . . . . . . . 38

3.4 Scheme of oscillating response with squared KWW-fits for high field and

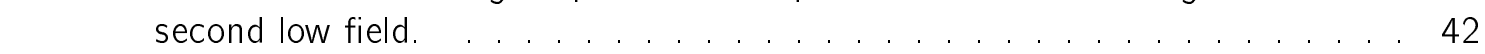

3.5 Scheme of oscillating excitation or response with positive ascending parts of each period marked by green rectangles. . . . . . . . . . . . . . . 43

3.6 An example for a positive ascending branch of strain data, and the resulting plot of strain $\varepsilon$ against intra-periodic stress $\sigma_{c}$. The green dotted line separates the number of data points into two halfs of same number of data

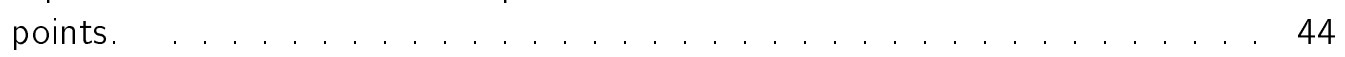

\begin{tabular}{ll|l|l|}
\hline 3.7 & Zoom on data shown in fig. & 3.6 & Magenta circles depict the first data point
\end{tabular} belonging to an interval of increasing slope (further on called a "positive" interval), equal to the end of an interval with negative slope (referred to as a "negative" interval). Grey circles thus depict the first point of a negative and the final data point of a positive interval. . . . . . . . . . . . 45

4.1 Normalized intensity / plotted against angle $\Theta$ for various samples. Samples $\mathrm{i}$ \begin{tabular}{|l}
\hline to $v$ measured before, samples vi and vii measured after mechanical high-field \\
\hline \hline experiments. . . . . . . . . . . . . . . . . . . . . . . 47
\end{tabular}

4.2 Plot of the specific heat contribution $\Delta c_{p}$ of the amorphous material against temperature $T$ derived from a DSC-measurement at a heating rate of $10 \mathrm{~K} \mathrm{~min}^{-1}$. Magenta and orange lines indicate linear fits to determine the onset of crystallization $T_{x}$ by the double-tangential method. . . . . . . . . . . . . . . 48

4.3 Plot of specific heat contribution $\Delta c_{p}$ of the amorphous material against temperature $T$ in the regime of the glass transition derived from a DSCmeasurement with a heating rate of $10 \mathrm{~K} \mathrm{~min}^{-1}$. Dolid lines correspond to linear fits to determine the glass transition temperature of the sample by the double tangential method. The red dotted line indicates the level of specific heat of the supercooled liquid, from which $\Delta c_{p}^{s-1}$ is determined to $236(2) \mathrm{mJg}^{-1} \mathrm{~K}^{-1} . \ldots \ldots \ldots \ldots . \ldots . \ldots . \ldots 49$

4.4 Static stress $\sigma_{s t}$ plotted against the static strain $\varepsilon_{s t}$ measured at two different temperatures. Dashed boxes indicate stress and strain regimes of LAOSmeasurements. . . . . . . . . . . . . . . . . . . . . . . . 50

4.5 Storage modulus (a) and loss modulus (b), $E^{\prime}$ and $E^{\prime \prime}$, plotted versus temperature $T$ in a strain-controlled measurement at a displacement amplitude of $1 \mu \mathrm{m}$ and a scanning rate of $2 \mathrm{~K} \mathrm{~min}^{-1}$. The green and grey dashed lines depict the onset of the glass transition $T_{g}^{o n}$ and the loss-peak temperature $T_{g}^{P}$ with shaded areas in green and grey according to the standard deviation. The red-colored background corresponds to the section of the temperature scan in which the sample response is compromised by crystallization.

4.6 Storage compliance (a) and loss compliance (b), $\chi^{\prime}$ and $\chi^{\prime \prime}$, plotted versus temperature $T$, determined according to eq. 3.12 from the modulus due to low field temperature scans (cf. fig|4.5). The green and grey dashed lines depict the onset of the glass transition $T_{q}^{o n}$ and the loss-peak temperature $T_{g}^{P}$ with shaded areas in green and grey according to the standard deviation. The red-colored background corresponds to the section of the temperature scan in which the sample response is compromised by crystallization. . . . . . 51

5.1 Lissajous-plots of stress $\hat{\sigma}$ and strain $\hat{\epsilon}$ for increasing stress amplitude $\sigma_{0}$ at a temperature of $563 \mathrm{~K}$. 
5.2 Lissajous-plots of stress $\hat{\sigma}$ and strain $\hat{\epsilon}$ for increasing temperature at a stress amplitude $\sigma_{0}$ of about $60 \mathrm{MPa} . \ldots \ldots \ldots \ldots 5$

5.3 Lissajous-plots of stress $\hat{\sigma}$ and strain $\hat{\epsilon}$ for periods with increasing experimental time during high field excitation at a temperature of $565 \mathrm{~K}$ and a stress amplitude of $37 \mathrm{MPa}$.

5.4 Comparison of temperature scans at low displacement amplitude (lines) and initial low field values from LAOS experiments (green symbols) for storage (a) and loss (b) compliance, $\chi^{\prime}$ and $\chi^{\prime \prime}$, plotted versus temperature T. Red symbols are steady state values under high field excitation extracted from LAOS experiments. Data from temperature scans corresponds to data shown in fig. 4.6 and $4.5 . T_{g}^{o n}$ and $T_{g}^{P}$ correspond to the temperatures of the onset of the glass transition and of the loss peak position.

5.5 Strain amplitudes for $1^{\text {st }}(\square), 2^{\text {nd }}(\bullet), 3^{\text {rd }}(\nabla), 4^{\text {th }}(\Delta)$, and $5^{\text {th }}(\bullet)$ harmonic frequency extracted by Fourier-analysis from LAOS-measurements performed at $565 \mathrm{~K}$ and a high field stress amplitude of $54 \mathrm{MPa}$. . . . . . . 58

5.6 Example for time-dependent behavior of nonlinear relative change in storage compliance at fundamental frequency $\Delta \ln \left(\varepsilon^{\prime} / \sigma_{0}\right)_{\omega}$ at $T=563 \mathrm{~K}$ and $\sigma_{0}=51 \mathrm{MPa} \ldots \ldots \ldots \ldots \ldots \ldots$

5.7 Steady state amplitude of relative nonlinear contribution to storage compliance at fundamental frequency during high field excitation $\left[\Delta \ln \left(\varepsilon^{\prime} / \sigma_{0}\right)_{\omega}\right]_{h f}$ determined by $\mid K W W$ fits based on eq. 3.24 plotted vs. squared high field stress amplitude $\sigma_{0}^{2}$ for several temperatures. Lines correspond to linear fits, shaded areas to the uncertainty of the respective fit. . . . . . . . . 66 60

5.8 Steady state amplitude of relative nonlinear contribution to storage compliance at fundamental frequency during second low field excitation $\left[\Delta \ln \left(\varepsilon^{\prime} / \sigma_{0}\right)_{\omega}\right]_{2^{\text {nd }}}$ If determined by $\mid K W W$ fits based on eq. 3.25 plotted vs. squared high field stress amplitude $\sigma_{0}^{2}$ for several temperatures. Lines correspond to linear fits, shaded areas to the uncertainty of the respective fit.

5.9 Relative nonlinear coefficients $\chi_{1}^{\prime(3)} / \chi^{\prime(1)}$ based on data shown in fig. 5.7 and fig. 5.8 plotted in logarithmic scaling against temperature $T$. High field and second low field data is fitted by linear regressions (dashed lines), see text for the resulting slopes and ordinates. . . . . . . . . . . . . . . . 66 61

5.10 Average timescales, $\tau_{\text {mean, }}, \tau_{h f}$, and $\tau_{2^{\text {nd }}}$, , based on high field and second low field relative nonlinear storage compliance $\Delta \ln \left(\varepsilon^{\prime} / \sigma_{0}\right)_{\omega}$ plotted versus temperature $T$. Green symbols with line correspond to literature data by [151].

5.11 Examples for time-dependent behavior of nonlinear relative change in loss compliance at fundamental frequency $\Delta \ln \left(\varepsilon^{\prime \prime} / \sigma_{0}\right)_{\omega}$. (a) corresponds to data measured at $T=563 \mathrm{~K}$ and $\sigma_{0}=51 \mathrm{MPa}$, typical for measurements in the temperature range of $563 \mathrm{~K}$ and $567 \mathrm{~K}$. (b) is based on a measurement at $553 \mathrm{~K}$ and $\sigma_{0}=79 \mathrm{MPa}$.

5.12 Steady state values of relative nonlinear contribution to loss compliance at fundamental frequency during high field excitation $\left[\Delta \ln \left(\varepsilon^{\prime \prime} / \sigma_{0}\right)_{\omega}\right]_{h f}$ determined by KWW-fits based on eq. 3.24 plotted vs. squared high field stress amplitude $\sigma_{0}^{2}$ for several temperatures. Lines correspond to linear fits, shaded areas to the uncertainty of the respective fit.

5.13 Steady state values of relative nonlinear contribution to loss compliance at fundamental frequency during second low field excitation $\left[\Delta \ln \left(\varepsilon^{\prime \prime} / \sigma_{0}\right)_{\omega}\right]_{2^{\text {nd }} / f}$ determined by KWW-fits based on eq. 3.25 plotted against squared high field stress amplitude $\sigma_{0}^{2}$ for different temperatures. Lines correspond to linear fits, shaded areas to the uncertainty of the respective fit. 
5.14 Relative nonlinear coefficients $\chi_{1}^{\prime \prime(3)} / \chi^{\prime \prime(1)}$ extracted from data shown in fig. 5.12 and fig. 5.13 plotted in logarithmic scaling against temperature $T$. High field and second low field data is fitted by linear regressions (lines), see text for slopes and ordinates.

5.15 Average timescales, $\tau_{h f}, \tau_{2^{\text {nd }}}$,f , and $\tau_{\text {mean }}$, based on high field and second low field relative nonlinear loss compliance $\Delta \ln \left(\varepsilon^{\prime \prime} / \sigma_{0}\right)_{\omega}$ plotted versus temperature $T$. Green symbols with line correspond to literature data by [151].

5.16 Example for time-dependent behavior of $\frac{\left(\epsilon / \sigma_{0}\right)_{3 \omega}}{\chi^{(1)}}$ at $T=563 \mathrm{~K}$ and $\sigma_{0}=10 \mathrm{MPa}$.

5.17 Steady state values of relative complex compliance at third harmonic frequency during high field excitation $\left[\left(\epsilon / \sigma_{0}\right)_{3 w} / \chi^{(1)}\right]_{h f}$ determined by KWWfits plotted against squared high field stress amplitude $\sigma_{0}^{2}$ for several temperatures. Lines correspond to linear fits, shaded areas to the uncertainty of the respective fit.

5.18 Relative nonlinear coefficient $\chi_{3}^{(3)} / \chi^{(1)}$ based on data shown in fig. $\mid 5.17$ plot-

ted against temperature $T$. The black dashed line resembles an exponential fit, see text for details on the fit function and for fit parameters.

5.19 Average timescales $\tau_{h f}$ of $\chi_{3 \omega} / \chi^{(1)}$ plotted versus temperature $T$. The green symbols with line correspond to literature data by [151]. . . . . . . . . . . . 69

5.20 Strain rate $\dot{\varepsilon}(\mathbf{a})$ and normalized strain rate $\dot{\varepsilon}(\mathbf{b})$ for various stress amplitudes $\sigma_{0}$ at constant temperature $T=563 \mathrm{~K}$.

5.21 Linearly plotted strain rate $\dot{\varepsilon}(\mathbf{a})$ and logarithmically plotted strain rate $\dot{\varepsilon}$ (b) against stress amplitude $\sigma_{0}$ for various temperatures. The black dashed line represents a fit based on data plotted as in (a) measured at $553 \mathrm{~K}$ extracted by linear regression.

5.22 Average of interval strain $\left\langle\left(\Delta \epsilon_{i t v l}\right)_{i j}\right\rangle_{c}$ in steady state for various stress amplitudes $\sigma_{0}$ at constant temperature $T=563 \mathrm{~K}$. (a) for positive and (b) for negative intervals.

5.23 Normalized average of interval strain $\left\langle\left(\Delta \epsilon_{i t v l}\right)_{i j}^{L}\right\rangle_{c}^{\text {norm }}$ for various stress amplitudes $\sigma_{0}$ at constant temperature $T=563 \mathrm{~K}$. (a) for positive and (b) for negative intervals.

5.24 Minimum (min) and maximum (max) values of interval strain, $\left\langle\left(\Delta \epsilon_{i t v l}\right)_{i j}^{L}\right\rangle^{\text {min }}$ and $<\left(\Delta \epsilon_{i t v l}\right)_{i j}^{\iota}>^{\text {Imax }}$,for various stress amplitudes $\sigma_{0}$ and temperatures $T$. (a) for positive and (b) for negative intervals. Green lines correspond to the mean value of $\left\langle\left(\Delta \epsilon_{i t v l}\right)_{i j}^{l}\right\rangle^{\text {min }}$, while the shaded area reflects the standard deviation.

5.25 Averages of interval duration $\left\langle\left(\Delta t_{i t v l}\right)_{i j}^{L}\right\rangle_{c}$ in steady state for various stress amplitudes $\sigma_{0}$ at constant temperature $T=563 \mathrm{~K}$. (a) for positive and (b) for negative intervals.

5.26 Normalized average interval duration $\left\langle\left(\Delta t_{i t v l}\right)_{i j}^{L}\right\rangle_{c}^{\text {norm }}$ for various stress amplitudes $\sigma_{0}$ at constant temperature $T=563 \mathrm{~K}$. (a) for positive and (b) for negative intervals.

5.27 Minimum and maximum values of interval duration, $\left\langle\left(\Delta t_{i t v l}\right)_{i j}^{L}\right\rangle^{\min }$ and $\left\langle\left(\Delta t_{i t v l}\right)_{i j}^{\iota}\right\rangle^{\text {IIdax }}$, for various stress amplitudes $\sigma_{0}$ and temperatures $T$. (a) for positive and (b) for neqative intervals. Green lines correspond to the mean value of $\left\langle\left(\Delta \epsilon_{i t v l}\right)_{i j}^{L}\right\rangle^{\min }$, while the shaded area reflects the standard deviation.

5.28 Double-logarithmic distribution of interval strain $\left(\Delta \epsilon_{i t v l}\right)^{\iota}$ for various stress amplitudes $\sigma_{0}$ at a constant temperature $T$ of $563 \mathrm{~K}$. Dashed-dotted lines correspond to maximum average strain values for measurements with a stress amplitude of $7 \mathrm{MPa}$ (black) and of $59 \mathrm{MPa}$ (gray). The dashed light gray line indicates the power-law regime. (a) for positive and (b) for negative intervals. 82 
5.29 Double-logarithmic distributions of interval strain $\left(\Delta \epsilon_{i t v l}\right)^{\iota}$ at a stress amplitude $\sigma_{0}$ of $7 \mathrm{MPa}$ comparing various temperatures $T$. The dashed light gray \begin{tabular}{|c|c|c|}
\hline \hline line indicates the power-law regime. (a) for positive and (b) for negative \\
\hline
\end{tabular} intervals. .

5.30 Double-logarithmic distributions of interval strain $\left(\Delta \epsilon_{i t v l}\right)^{L}$ at a stress amplitude $\sigma_{0}$ of $60 \mathrm{MPa}$ comparing various temperatures $T$. The dashed light gray line indicates the power-law regime. (a) for positive and (b) for negative intervals. .

5.31 Exponent $\kappa$ based on the linear regime of double-logarithmic distribution on interval strain $\left\langle\left(\Delta \epsilon_{i t v l}\right)_{i j}^{\iota}\right\rangle$ (cf. e.g. fig. 5.29 and fig. 5.30). (a) for positive and (b) for negative intervals.

6.1 Scheme of the contributions resulting in the overall nonlinear strain response (solid red line) for one period. The solid blue line depicts the linear contribution of strain response, the red dashed line is due to both linear and nonlinear contribution at the first harmonic frequency, the red dotted line is based on the contribution at the third harmonic. Amplitudes and phase-shifts reflect the relations of the measurement shown in fig. $5.5 \square \ldots \ldots 90$

6.2 Relative nonlinear coefficients for first harmonic storage and loss, and third harmonic complex contribution plotted against temperature $T . \ldots 92$

6.3 Scheme of the different effects of temperature $T$ and mechanical excitation (here referred to as $\Sigma$ ) from [101].

6.4 Temperature-dependent timescales for first harmonic storage and loss compliance, $\tau_{\chi^{\prime}, 1 \omega}^{h f}$ and $\tau_{\chi^{\prime \prime}, 1 \omega}^{h f}$, and third harmonic complex compliance $\tau_{\chi, 3 \omega}^{h f}$ under high field excitation as in figures 5.10 . 55.15 and 5.19 depicted by symbols. Literature data on the structural relaxation timescale $\tau_{\alpha}$ [151] correspond to green symbols connected by lines. The blue dashed line serves as a guide to the eye for the general trend of the measured recovery timescales with temperature.

6.5 Normalized strain rate $\dot{\varepsilon}_{\text {norm }}$ and normalized averaged interval strain $\left\langle\left(\Delta \epsilon_{i t v l}\right)_{i j}^{L}>_{c}^{n o}{ }^{m}\right.$ plotted against the normalized intra-periodic stress $\sigma_{c}$ for a stress amplitude of $\sigma_{0}=60 \mathrm{MPa}$ at constant temperature $T=563 \mathrm{~K} . \ldots \ldots 6$

6.6 Normalization factor of the averaged interval strain plotted against the normalization factor of the strain rate for a stress amplitude of $\sigma_{0}=60 \mathrm{MPa}$ at constant temperature $T=563 \mathrm{~K} . \ldots \ldots \ldots 9 . \ldots \ldots$

6.7 (a) normalized strain rate $\dot{\varepsilon}_{\text {norm }}$ and normalized averaged interval duration $<\left(\Delta t_{i t v l}\right)_{i j}^{L}>_{c}^{\text {norm }}$ plotted against normalized intra-periodic stress $\sigma_{c}$. (b) normalization factor of the averaged interval duration plotted against the normalization factor of the strain rate. Both plots are shown for a stress amplitude of $\sigma_{0}=60 \mathrm{MPa}$ at constant temperature $T=563 \mathrm{~K} . \ldots 97$

6.8 Zoom of the hump of the interval strain distributions for positive intervals as shown in fig. 5.28 for various stress amplitude $\sigma_{0}$ at constant temperature $T=563 \mathrm{~K}$. The arrow depicts schematically the observed shift of the hump with increasing stress amplitude $\sigma_{0}$, i.e. increasing strain rate $\dot{\varepsilon}$. . . . . . . 999

A.1 Counter-acting force due to spring stiffness and damping $F$

C.1 Second harmonic strain amplitude divided by squared stress amplitude $\left(\varepsilon / \sigma_{0}^{2}\right)_{2 \omega}$ plotted against static stress $\sigma_{s t}$ for different temperatures.

D.1 Relative nonlinear coefficient $\chi_{1}^{(5)} / \chi^{(1)}$ plotted in logarithmic scaling against temperature $T$. 
F.1 Normalized averaged interval compliance $\left\langle\left(\xi_{i t v l}\right)_{i j}^{P}\right\rangle^{\text {norm }}$ in comparison the the normalized data point density $\rho_{D P}$ plotted against the normalized intraperiodic stress $\sigma_{c} / \sigma_{n}$, as exemplification on positive strain intervals. . . . . . 132

F.2 Normalized averaged interval width $\left\langle\left(\Delta \sigma_{i t v l}\right)_{i j}^{P}>^{n o r m}\right.$ in comparison the the normalized data point density $\rho_{D P}$ plotted against the normalized intraperiodic stress $\sigma_{c} / \sigma_{0}$, as exemplification on positive strain intervals. . . . . . 133

F.3 Normalized umber of intervals $\left(N_{i t v l}\right)_{c} / \Sigma_{c}\left(N_{i t v l}\right)_{c}$ in comparison the the normalized data point density $\rho_{D P}$ plotted against the normalized intra-periodic

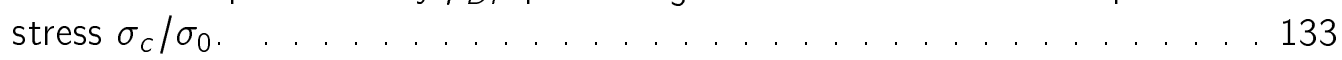

G.1 (a) Distribution of increments in sinusoidal data for the full positive ascending part of the sine, based on $\Delta \sigma_{i n c r}$ for various stress amplitudes $\sigma_{0}$. (b) Exponent $\lambda$ extracted from distributions shown in (a) plotted against stress

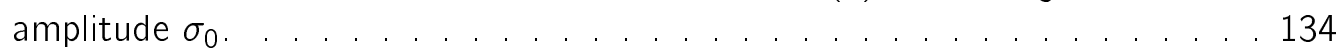

G.2 Distribution of increments in sinusoidal data for the initial 2/3 of the positive ascending part of the sine, based on $\Delta \sigma_{\text {incr }}$ occurring within the first two thirds of the stress amplitude (i.e. between stresses from 0 to $2 / 3 \cdot \sigma_{0}$ ) for various stress amplitudes $\sigma_{0} . \ldots \ldots \ldots 135$

G.3 Distribution of intervals in sinusoidal data, based on $\Delta \sigma_{i t v l}$ occurring within the first two thirds of the stress amplitude (i.e. between stresses from 0 to $\left.2 / 3 \cdot \sigma_{0}\right)$ for various stress amplitudes $\left.\sigma_{0}\right] \ldots \ldots \ldots \ldots$ 


\section{Acronyms}

CRR cooperative rearranging regions.

CSM cooperative shear model.

DES dielectric spectroscopy.

DMA dynamical mechanical analyzer.

DSC differential scanning calorimetry.

EDX energy dispersive X-Ray spectroscopy.

KWW Kohlrausch-Williams-Watts.

LAOS large amplitude oscillatory spectroscopy.

LVDT linear variable differential transformer.

MD molecular dynamics.

PEL potential energy landscape.

PMMA Polymethylmethacrylate.

SEM scanning electron microscope.

STZ shear transformation zone.

VFT Vogel-Fulcher-Tammann.

XRD X-Ray diffraction. 


\section{Bibliography}

[1] Gerold Adam and Julian H. Gibbs. On the temperature dependence of cooperative relaxation properties in glass-forming liquids. The Journal of Chemical Physics, 43(1):139-146, 1965.

[2] S. Albert, Thomas Bauer, Marion Michl, Giulio Biroli, Jean-Philippe Bouchaud, Alois Loidl, Peter Lunkenheimer, R. Tourbot, C. Wiertel-Gasquet, and F. Ladieu. Fifthorder susceptibility unveils growth of thermodynamic amorphous order in glass-formers. Science, 352(6291):1308-1311, 2016.

[3] C. Austen Angell. Perspective on the glass transition. J. Phys. Chem. Solids, 49(8):863 $-871,1988$.

[4] C. Austen Angell. Formation of glasses from liquids and biopolymers. Science, 267:1924-1935, 1995.

[5] AS Argon. Plastic deformation in metallic glasses. Acta metallurgica, 27(1):47-58, 1979.

[6] AS Argon and L To Shi. Development of visco-plastic deformation in metallic glasses. Acta Metallurgica, 31(4):499-507, 1983.

[7] Per Bak, Chao Tang, and Kurt Wiesenfeld. Self-organized criticality: An explanation of the 1/f noise. Phys. Rev. Lett., 59:381-384, 1987.

[8] Thomas Bauer, Peter Lunkenheimer, and Alois Loidl. Cooperativity and the freezing of molecular motion at the glass transition. Phys. Rev. Lett., 111:225702, 2013.

[9] Dennis Bedorf and Konrad Samwer. Length scale effects on relaxations in metallic glasses. Journal of Non-Crystalline Solids, 356:340 - 343, 2010.

[10] Martin S Beevers, David Alan Elliott, and Graham Williams. Static and dynamic kerreffect studies of glycerol in its highly viscous state. Journal of the Chemical Society, Faraday Transactions 2: Molecular and Chemical Physics, 76:112-121, 1980.

[11] Ludovic Berthier and Jean-Louis Barrat. Shearing a glassy material: Numerical tests of nonequilibrium mode-coupling approaches and experimental proposals. Phys. Rev. Lett., 89:095702, c 2002.

[12] Roland Böhmer and C. Austen Angell. Correlations of the nonexponentiality and state dependence of mechanical relaxations with bond connectivity in ge-as-se supercooled liquids. Physical Review B, 45:10091-10094, n 1992.

[13] Roland Böhmer, R.V. Chamberlin, G. Diezemann, B. Geil, A. Heuer, G. Hinze, S.C. Kuebler, Ranko Richert, B. Schiener, H. Sillescu, H.W. Spiess, U. Tracht, and M. Wilhelm. Nature of the non-exponential primary relaxation in structural glass-formers probed by dynamically selective experiments. Journal of Non-Crystalline Solids, 235237:1 - 9, 1998. 
[14] Roland Böhmer, K. L. Ngai, C. Austen Angell, and D. J. Plazek. Nonexponential relaxations in strong and fragile glass formers. The Journal of Chemical Physics, 99(5):4201-4209, 1993.

[15] Max Born and Kun Huang. Dynamical theory of crystal lattices. Clarendon press, 1954.

[16] Jean-Philippe Bouchaud and Giulio Biroli. Nonlinear susceptibility in glassy systems: A probe for cooperative dynamical length scales. Physical Review B, 72:064204, 2005.

[17] R. F. Boyer. Dependence of mechanical properties on molecular motion in polymers. Polymer Engineering \& Science, 8(3):161-185, 1968.

[18] C Brun, F Ladieu, D I'Hôte, M Tarzia, Giulio Biroli, and Jean-Philippe Bouchaud. Nonlinear dielectric susceptibilities: accurate determination of the growing correlation volume in a supercooled liquid. Physical Review B, 84(10):104204, 2011.

[19] V. Chikkadi, S. Mandal, B. Nienhuis, D. Raabe, F. Varnik, and P. Schall. Shearinduced anisotropic decay of correlations in hard-sphere colloidal glasses. Europhys. Lett., 100(5):56001, 2012.

[20] Vijayakumar Chikkadi, Gerard Wegdam, Daniel Bonn, Bernard Nienhuis, and Peter Schall. Long-range strain correlations in sheared colloidal glasses. Phys. Rev. Lett., 107:198303, 2011.

[21] R. M. Christensen. Theory of Viscoelasticity, An Introduction. Academic NY, 2nd edition edition, 1982

[22] Yossi Cohen, Smarajit Karmakar, Itamar Procaccia, and Konrad Samwer. The nature of the beta-peak in the loss modulus of amorphous solids. Europhys. Lett., 100(3):36003, 2012 .

[23] C. Crauste-Thibierge, C. Brun, F. Ladieu, D. L'Hôte, Giulio Biroli, and Jean-Philippe Bouchaud. Evidence of growing spatial correlations at the glass transition from nonlinear response experiments. Phys. Rev. Lett., 104:165703, I 2010.

[24] Karin A Dahmen and Yehuda Ben-Zion. Jerky motion in slowly driven magnetic and earthquake fault systems, physics of. In Encyclopedia of Complexity and Systems Science, pages 5021-5037. Springer, 2009.

[25] Vladimir Dailidonis, Valery llyin, Itamar Procaccia, and Carmel ABZ Shor. Breakdown of nonlinear elasticity in stress-controlled thermal amorphous solids. Physical Review E, 95(031001(R)), 2017.

[26] R. de L. Kronig. On the theory of dispersion of x-rays. J. Opt. Soc. Am., 12(6):547557, 1926.

[27] Pablo G. Debenedetti and Frank H. Stillinger. Supercooled liquids and the glass trasition. Nature, 410:259-267, 2001.

[28] P Debye. The theory of anomalous dispersion in the region of long-wave electromagnetic radiation. Verhandlungen deutsche physikalische Gesellschaft, 15:777-793, 1913.

[29] Peter Josef William Debye. Polar molecules. Chemical Catalog Company, Incorporated, 1929.

[30] W. Demtröder. Experimentalphysik 1. Experimentalphysik / Wolfgang Demtröder. Springer Berlin Heidelberg, 2001. 
[31] Claudio Donati, Jack F. Douglas, Walter Kob, Steven J. Plimpton, Peter H. Poole, and Sharon C. Glotzer. Stringlike cooperative motion in a supercooled liquid. Phys. Rev. Lett., 80:2338-2341, 1998.

[32] Awadhesh K. Dubey, Itamar Procaccia, Carmel A. B. Z. Shor, and Murari Singh. Elasticity in amorphous solids: Nonlinear or piecewise linear? Phys. Rev. Lett., 116:085502, 2016.

[33] Gianfranco Durin and Stefano Zapperi. Scaling exponents for barkhausen avalanches in polycrystalline and amorphous ferromagnets. Phys. Rev. Lett., 84(20):4705, 2000.

[34] Jeppe C Dyre. Colloquium: The glass transition and elastic models of glass-forming liquids. Reviews of Modern Physics, 78(3):953, 2006.

[35] M D Ediger. Spatially heterogeneous dynamics in supercooled liquids. Annu. Rev. Phys. Chem., 51(1):99-128, 2000.

[36] Mark D. Ediger., C. Austen Angell, and Sidney R. Nagel. Supercooled liquids and glasses. The Journal of Physical Chemistry, 100(31):13200-13212, 1996.

[37] Peter Ehrenfest. Leiden Comm. Suppl., 75b, 1933.

[38] S R Elliott. Physics of Amorphous Materials. Longman, 1983.

[39] Perkin Elmer. Dma 8000 booklet, 2007.

[40] Perkin Elmer. Dma 8000 sample kit guide, 2007.

[41] JD Eshelby. The elastic field outside an ellipsoidal inclusion. In Proceedings of the Royal Society of London A: Mathematical, Physical and Engineering Sciences, volume 252, pages 561-569. The Royal Society, 1959.

[42] John D Eshelby. The determination of the elastic field of an ellipsoidal inclusion, and related problems. In Proceedings of the Royal Society of London A: Mathematical, Physical and Engineering Sciences, volume 241, pages 376-396. The Royal Society, 1957.

[43] Franz Faupel, Werner Frank, Michael-Peter Macht, Helmut Mehrer, Volkmar Naundorf, Klaus Rätzke, Herbert R. Schober, Suman K. Sharma, and Helmar Teichler. Diffusion in metallic glasses and supercooled melts. Reviews of Modern Physics, 75:237280, 2003.

[44] Stefanie Finkhäuser. Mechanische Spektroskopie an PMMA-Systemen: Nichtlineares Verhalten und UV-aktive Blockcopolymere. PhD thesis, Georg-August-Universität Göttingen, 2017.

[45] W. Flügge. Viscoelasticity. Springer-Verlag NY, 2nd ed. edition, 1975.

[46] G. S. Fulcher. Analysis of recent measurements of the viscosity of glasses. J. Am. Ceram. Soc., 8:339-355, 1925.

[47] Clemens Garve. Mechanische analyse von palladium-basierten metallischen gläsern im nichtlinearen spannungs-dehnungs-bereich. Master's thesis, I. Physikalisches Institut, Georg-August-Universität, 2017.

[48] Martin Goldstein. Viscous liquids and the glass transition: A potential energy barrier picture. The Journal of Chemical Physics, 51(9):3728-3739, 1969.

[49] A.L. Greer, Y.Q. Cheng, and E. Ma. Shear bands in metallic glasses. Materials Science and Engineering: R: Reports, 74(4):71 - 132, 2013.

[50] Pengfei Guan, Mingwei Chen, and Takeshi Egami. Stress-temperature scaling for steady-state flow in metallic glasses. Phys. Rev. Lett., 104:205701, 2010. 
[51] Peter Haasen. Physikalische Metallkunde. Springer-Verlag, 3 edition, 1994.

[52] John S. Harmon, Marios D. Demetriou, William L. Johnson, and Konrad Samwer. Anelastic to plastic transition in metallic glass-forming liquids. Phys. Rev. Lett., 99:135502, 2007.

[53] William M. Haynes. CRC Handbook of Chemistry and Physics. Taylor \& Francis, 96 edition, 2015.

[54] Carlos Herrero-Gómez and Konrad Samwer. Stress and temperature dependence of the avalanche dynamics during creep deformation of metallic glasses. Scientific Reports, 6(33503), 2016.

[55] Andreas Heuer. Exploring the potential energy landscape of glass-forming systems: from inherent structures via metabasins to macroscopic transport. J. Phys.: Condens. Matter, 20(37):373101, 2008.

[56] Lina Hu, Xiufang Bian, Weimin Wang, Guangrong Liu, and Yubo Jia. Thermodynamics and dynamics of metallic glass formers:? their correlation for the investigation on potential energy landscape. The Journal of Phys. Chem. B, 109(28):13737-13742, 2005. PMID: 16852721.

[57] Wei Huang and Ranko Richert. The physics of heating by time-dependent fields: Microwaves and water revisited. The Journal of Physical Chemistry B, 112(32):99099913, 2008.

[58] Wei Huang and Ranko Richert. Dynamics of glass-forming liquids. xiii. microwave heating in slow motion. The Journal of Chemical Physics, 130(19):-, 2009.

[59] Todd C Hufnagel, Christopher A Schuh, and Michael L Falk. Deformation of metallic glasses: Recent developments in theory, simulations, and experiments. Acta Mater., 109:375-393, 2016.

[60] NORAN Instruments. Energy-dispersive x-ray microanalysis - an introduction. 2551 West BeltlineHighway Middleton, WI 53562, 1999.

[61] Prabhat K. Jaiswal, Itamar Procaccia, Corrado Rainone, and Murari Singh. Mechanical yield in amorphous solids: A first-order phase transition. Phys. Rev. Lett., 116:085501, 2016.

[62] Gyan P. Johari and Martin Goldstein. Viscous liquids and the glass transition. ii. secondary relaxations in glasses of rigid molecules. The Journal of Chemical Physics, 53(6):2372-2388, 1970.

[63] William L Johnson, Marios D Demetriou, John S Harmon, Mary L Lind, and Konrad Samwer. Rheology and ultrasonic properties of metallic glass-forming liquids: A potential energy landscape perspective. MRS bulletin, 32(08):644-650, 2007.

[64] WL Johnson and Konrad Samwer. A universal criterion for plastic yielding of metallic glasses with a (t/t g) 2/3 temperature dependence. Phys. Rev. Lett., 95(19):195501, 2005.

[65] W. Klement Jun, R. H. Willens, and Pol Duwez. Non-crystalline structure in solidsolid gold-silicon alloys. Nature, 187:869-870, 1960.

[66] A. Kahl, T. Koeppe, Dennis Bedorf, Ranko Richert, M.L. Lind, M.D. Demetriou, W.L. Johnson, Walter Arnold, and Konrad Samwer. Dynamical and quasistatic structural relaxation paths in pd40ni40p20 glass. Appl. Phys. Lett., 95:201903, 2009.

[67] Yoshihito Kawamura and Akihisa Inoue. Newtonian viscosity of supercooled liquid in a pd40ni40p20 metallic glass. Appl. Phys. Lett., 77(8):1114-1116, 2000. 
[68] Charles Kittel. Introduction to Solid State Physics. John Wiley \& Sons, Inc, 8 edition, 2005.

[69] R Kohlrausch. Theory of the electrical residue in the leiden bottle. Pogg. Ann. Phys. Chem, 91:179-214, 1854.

[70] H.A. Kramers. Brownian motion in a field of force and the diffusion model of chemical reactions. Physica, 7(4):284-304, 1940.

[71] Hendrik Anthony Kramers. La diffusion de la lumiere par les atomes. 1927.

[72] J Krausser, AE Lagogianni, K Samwer, and A Zaccone. Disentangling interatomic repulsion and anharmonicity in the viscosity and fragility of glasses. Physical Review B, 95(10):104203, 2017.

[73] Johannes Krausser, Konrad H. Samwer, and Alessio Zaccone. Interatomic repulsion softness directly controls the fragility of supercooled metallic melts. Proceedings of the National Academy of Sciences, 112(45):13762-13767, 2015.

[74] Jon-Olaf Krisponeit, Sebastian Pitikaris, Karina E. Avila, Stefan Küchemann, Antje Krüger, and Konrad Samwer. Crossover from random three-dimensional avalanches to correlated nano shear bands in metallic glasses. Nature Communications, 5(3616):5, 2013.

[75] Stefan Küchemann and Konrad Samwer. Ultrafast heating of metallic glasses reveals disordering of the amorphous structure. Acta Mater., 104:119-124, 2016.

[76] Alexandra E Lagogianni, Johannes Krausser, Zach Evenson, Konrad Samwer, and Alessio Zaccone. Unifying interatomic potential, g ( $r$ ), elasticity, viscosity, and fragility of metallic glasses: analytical model, simulations, and experiments. Journal of Statistical Mechanics: Theory and Experiment, 2016(8):084001, 2016.

[77] Alexandra E. Lagogianni and Konrad Samwer. Eip-motif. Private Communications, 2017.

[78] John Lamb. Viscoelasticity and lubrication: a review of liquid properties. Journal of Rheology, 22(4):317-347, 1978.

[79] E. F. Lambson, W. A. Lambson, J. E. Macdonald, M. R. J. Gibbs, G. A. Saunders, and D. Turnbull. Elastic behavior and vibrational anharmonicity of a bulk $\mathrm{pd}_{40} \mathrm{ni}_{40} \mathrm{p}_{20}$ metallic glass. Physical Review B, 33:2380-2385, 1986.

[80] Antoine Le Bouil, Axelle Amon, Sean McNamara, and Jérôme Crassous. Emergence of cooperativity in plasticity of soft glassy materials. Phys. Rev. Lett., 112(24):246001, 2014.

[81] Premkumar Leishangthem, Anshul DS Parmar, and Srikanth Sastry. The yielding transition in amorphous solids under oscillatory shear deformation. Nature Communications, 8(14653):8, 2017.

[82] Anaël Lemaître and Christiane Caroli. Rate-dependent avalanche size in athermally sheared amorphous solids. Phys. Rev. Lett., 103:065501, 2009.

[83] Frederick A Lindemann. Ueber die berechnung molekularer eigenfrequenzen. Phys. Z, 11:609-612, 1910.

[84] P Lunkenheimer, M Michl, Th Bauer, and A Loidl. Investigation of nonlinear effects in glassy matter using dielectric methods. arXiv preprint arXiv:1704.07348, 2017.

[85] Peter Lunkenheimer, U. Schneider, R. Brand, and Alois Loidl. Glassy dynamics. Contemporary Physics, 41(1):15-36, 2000. 
[86] Robert Maaß and Jörg F Löffler. Shear-band dynamics in metallic glasses. Adv. Funct. Mater., 25(16):2353-2368, 2015.

[87] Robert Maaß, Konrad Samwer, Walter Arnold, and CA Volkert. A single shear band in a metallic glass: Local core and wide soft zone. Appl. Phys. Lett., 105(17):171902, 2014.

[88] Craig Maloney and Anaël Lemaitre. Subextensive scaling in the athermal, quasistatic limit of amorphous matter in plastic shear flow. Phys. Rev. Lett., 93:016001, 2004.

[89] Craig Maloney and Anael Lemaitre. Universal breakdown of elasticity at the onset of material failure. Phys. Rev. Lett., 93:195501, 2004.

[90] J. Clerk Maxwell. On the dynamical theory of gases. Philos. Trans. Roy. Soc. London, 157:49-88, 1867.

[91] Sean McNamara, Jérôme Crassous, and Axelle Amon. Eshelby inclusions in granular matter: Theory and simulations. Physical Review E, 94:022907, u 2016.

[92] Kevin P. Menard. Dyamic Mechanical Analysis: A Practical Introduction. CRC Press LLC, 1999.

[93] M Michl, Th Bauer, P Lunkenheimer, and A Loidl. Cooperativity and heterogeneity in plastic crystals studied by nonlinear dielectric spectroscopy. Phys. Rev. Lett., 114(6):067601, 2015.

[94] CT Moynihan, LP Boesch, and NL Laberge. Decay function for electric-field relaxation in-vitreous ionic conductors. Phys. Chem. Glasses, 14(6):122-125, 1973.

[95] C.T. Moynihan and J. Schroeder. Non-exponential structural relaxation, anomalous light scattering and nanoscale inhomogeneities in glass-forming liquids. J. Non-Cryst. Solids, 160(1):52 - 59, 1993.

[96] Toshiji Mukai, T.G Nieh, Yoshihito Kawamura, Akihisa Inoue, and Kenji Higashi. Dynamic response of a pd40ni40p20 bulk metallic glass in tension. Scripta Materialia, 46(1):43-47, 2002.

[97] K. L. Ngai and M. Paluch. Classification of secondary relaxation in glass-formers based on dynamic properties. The Journal of Chemical Physics, 120(2):857-873, 2004.

[98] KL Ngai. Relation between some secondary relaxations and the $\alpha$ relaxations in glassforming materials according to the coupling model. The Journal of Chemical Physics, 109(16):6982-6994, 1998.

[99] KL Ngai. An extended coupling model description of the evolution of dynamics with time in supercooled liquids and ionic conductors. J. Phys.: Condens. Matter, 15(11):S1107, 2003.

[100] KL Ngai and KL Ngai Comm. Solid state phys., 9 (1979). Comm. Solid State Phys, 9:141, 1980.

[101] Alexandre Nicolas, Kirsten Martens, and Jean-Louis Barrat. Rheology of athermal amorphous solids: Revisiting simplified scenarios and the concept of mechanical noise temperature. Europhys. Lett., 107(4):44003, 2014.

[102] V. N. Novikov and A. P. Sokolov. Correlation of fragility and poisson's ratio: Difference between metallic and nonmetallic glass formers. Physical Review B, 74:064203, 2006.

[103] PA O'connell and GB McKenna. Large deformation response of polycarbonate: Timetemperature, time-aging time, and time-strain superposition. Polymer Engineering \& Science, 37(9):1485-1495, 1997. 
[104] D Pan, A Inoue, T Sakurai, and Mingwei Chen. Experimental characterization of shear transformation zones for plastic flow of bulk metallic glasses. Proceedings of the National Academy of Sciences, 105(39):14769-14772, 2008.

[105] Perkin Elmer. DMA 8000 Service Manual and Software Program, 2007.

[106] Olga Perković, Karin Dahmen, and James P. Sethna. Avalanches, barkhausen noise, and plain old criticality. Phys. Rev. Lett., 75:4528-4531, 1995.

[107] SD Poisson. Note sur l'extension des fils et des plaques élastiques. In Annales de Chimie et de Physique, volume 36, pages 384-387, 1827.

[108] DE Polk and D Turnbull. Flow of melt and glass forms of metallic alloys. Acta Metallurgica, 20(4):493-498, 1972.

[109] T. Pritz. Verification of local kramers-kronig relations for complex modulus by means of fractional derivative model. Journal of Sound and Vibration, 228(5):1145 - 1165, 1999.

[110] Itamar Procaccia, Corrado Rainone, Carmel A. B. Z. Shor, and Murari Singh. Breakdown of nonlinear elasticity in amorphous solids at finite temperatures. Physical Review E, 93:063003, 2016.

[111] Ranko Richert. Homogeneous dispersion of dielectric responses in a simple glass. J. Non-Cryst. Solids, 172:209 - 213, 1994.

[112] Ranko Richert. Heterogeneous dynamics in liquids: fluctuations in space and time. J. Phys.: Condens. Matter, 14(23):R703, 2002.

[113] Ranko Richert. Frequency dependence of dielectric saturation. Physical Review E, 88(6):062313, 2013.

[114] Ranko Richert. Reponse estimation for pd-based metallic glass calcuated on the basis of the box-model. Private Communications, 2014.

[115] Ranko Richert. Non-linear dielectric signatures of entropy changes in liquids subject to time dependent electric fields. The Journal of Chemical Physics, 144(11):114501, 2016.

[116] Ranko Richert and Wei Huang. Time-resolved non-linear dielectric responses in molecular systems. Journal of Non-Crystalline Solids, 356(11-17):787 - 793, 2010. Proceedings of the 5th International Conference on Broadband Dielectric Spectroscopy and its Applications.

[117] Ranko Richert and Susan Weinstein. Nonlinear dielectric response and thermodynamic heterogeneity in liquids. Phys. Rev. Lett., 97:095703, t 2006.

[118] Birte Riechers, Konrad Samwer, and Ranko Richert. Structural recovery in plastic crystals by time-resolved non-linear dielectric spectroscopy. The Journal of Chem. Phys., 142(15):-, 2015.

[119] P. Rösner, Konrad Samwer, and Peter Lunkenheimer. Indications for an "excess wing" in metallic glasses from the mechanical loss modulus in zr 65 al $7.5 \mathrm{cu} 27.5$. Europhys. Lett., 68(2):226, 2004.

[120] P. Rösner, M. Weiss, S. Schneider, and Konrad Samwer. Dynamic heterogeneities in the glassy and undercooled states of the amorphous system $\mathrm{Zr}_{65} \mathrm{al}_{x} \mathrm{Cu}_{35-x}$. J. NonCryst. Solids, 307-310:848 - 852, 2002.

[121] Stefan M. Sarge, Günther W. H. Höhner, and Wolfgang Hemminger. Calorimetry Fundamentals, Instrumentation and Applications. Wiley-VCH, 2014. 
[122] Peter Schall, David A. Weitz, and Frans Spaepen. Structural rearrangements that govern flow in colloidal glasses. Science, 318(5858):1895-1899, 2007.

[123] Felix Schlenkrich, Susanne Seyffarth, Britta Fuchs, and Hans-Ulrich Krebs. Pulsed laser deposition of polymer-metal nanocomposites. Appl. Surf. Sci., 257(12):5362-5365, 2011.

[124] H.R. Schober. Soft phonons in glasses. Physica A: Statistical Mechanics and its Applications, 201:14 - 24, 1993.

[125] K. Schröter, Gerhard Wilde, R. Willnecker, Manfred Weiss, Konrad Samwer, and $E$. Donth. Shear modulus and compliance in the range of the dynamic glass transition for metallic glasses. The European Physical Journal B - Condensed Matter and Complex Systems, 5(1):1-5, 1998.

[126] Moritz Schwabe, Dennis Bedorf, and Konrad Samwer. Influence of stress and temperature on damping behavior of amorphous pd77.5cu6.0si16.5 below tg. The European Physical Journal E, 34(9):91, 2011.

[127] Moritz Schwabe, Stefan Küchemann, Hannes Wagner, Dennis Bedorf, and Konrad Samwer. Activation volume of microscopic processes in amorphous pd $77.5 \mathrm{cu} 6.0 \mathrm{si}$ 16.5 due to stress and temperature. J. Non-Cryst. Solids, 357(2):490-493, 2011.

[128] S.D. Senturia. Microsystem Design. Springer US, 2007.

[129] LT Shi, AS Argon, and HY Kuo. Effects of stabilization on the elastic to visco-plastic transition in metallic glasses. Scripta metallurgica, 17(8):1015-1020, 1983.

[130] Frans Spaepen. A microscopic mechanism for steady state inhomogeneous flow in metallic glasses. Acta metallurgica, 25(4):407-415, 1977.

[131] H. Eugene Stanley. Scaling, uniuniversal, and renormalization: Three pillars of modern critical phenomena. Reviews of Modern Physics, 71(2):358-366, 1999.

[132] Jacob D. Stevenson, Jörg Schmalian, and Peter G. Wolynes. The shapes of cooperatively rearranging regions in glass-forming liquids. Nature Physics, 2:268-274, 2006.

[133] F. H. Stillinger. A topographic view of supercooled liquids and glass formation. Science, 267:1935-1939, 1995.

[134] BA Sun, S Pauly, J Tan, M Stoica, Wei Hua Wang, U Kühn, and Jürgen Eckert. Serrated flow and stick-slip deformation dynamics in the presence of shear-band interactions for a zr-based metallic glass. Acta Mater., 60(10):4160-4171, 2012.

[135] Bosiljka Tadić. Nonuniversal scaling behavior of barkhausen noise. Phys. Rev. Lett., 77(18):3843, 1996.

[136] G. Tammann and W. Hesse. Die abhängigkeit der viscosität von der temperatur bei unterkühlten flüssigkeiten. Zeitschrift für anorganische und allgemeine Chemie, 156:245-257, 1926.

[137] A. Tanguy, F. Leonforte, and J. L. Barrat. Plastic response of a 2d lennard-jones amorphous solid: Detailed analysis of the local rearrangements at very slow strain rate. The European Physical Journal E, 20(3):355-364, 2006.

[138] Marco Tarzia, Giulio Biroli, Alexandre Lefòvre, and Jean-Philippe Bouchaud. Anomalous nonlinear response of glassy liquids: General arguments and a mode-coupling approach. The Journal of Chemical Physics, 132(5):054501-1:10, 2010.

[139] $\mathrm{H}$. Teichler. Heterogeneous dynamics on the microsecond scale in simulated $\mathrm{ni}_{0.5} \mathrm{Zr}_{0.5}$ metallic melts far below the glass temperature. Physical Review E, 71:031505, a 2005. 
[140] Alex Travesset, Robert A White, and Karin A Dahmen. Crackling noise, power spectra, and disorder-induced critical scaling. Physical Review B, 66(2):024430, 2002.

[141] Michel Tsamados. Plasticity and dynamical heterogeneity in driven glassy materials. The European Physical Journal E: Soft Matter and Biological Physics, 32(2):165-181, 2010.

[142] Michel Tsamados, Anne Tanguy, Fabien Léonforte, and J-L Barrat. On the study of local-stress rearrangements during quasi-static plastic shear of a model glass: Do local-stress components contain enough information? The European Physical Journal E, 26(3):283-293, 2008.

[143] H. Vogel. Dastemperaturabhängigkeitsgesetz der viskosität von flüssigkeiten. Phys. Z, 22:645-646, 1921.

[144] M. Vogel and E. Rössler. On the nature of slow $\beta$-process in simple glass formers: A 2h nmr study. The Journal of Physical Chemistry B, 104(18):4285-4287, 2000.

[145] Hannes Wagner, Dennis Bedorf, Stefan Küchemann, Moritz Schwabe, Bo Zhang ans Walter Arnold, and Konrad Samwer. Local elastic properties of a metllic glass. Nat. Mater., 10:439-442, 2011.

[146] Eric R. Weeks, J. C. Crocker, Andrew C. Levitt, Andrew Schofield, and D. A. Weitz. Three-dimensional direct imaging of structural relaxation near the colloidal glass transition. Science, 287(5453):627-631, 2000.

[147] Susan Weinstein and Ranko Richert. Nonlinear features in the dielectric behavior of propylene glycol. Physical Review B, 75(6):064302, 2007.

[148] Susan Weinstein and Ranko Richert. Probing heterogeneous thermal relaxation by nonlinear dielectric spectroscopy. J. Phys.: Condens. Matter, 19(20):205128, 2007.

[149] Manfred Weiß. Mechanische Eigenschaften und thermische Stabilität tief unterküh/ter Metallschmelzen im Bereich der Glastemperatur. PhD thesis, Universität Augsburg, 1998.

[150] Robert A White and Karin A Dahmen. Driving rate effects on crackling noise. Phys. Rev. Lett., 91(8):085702, 2003.

[151] Gerhard Wilde, G. P. Görler, R. Willnecker, and H. J. Fecht. Calorimetric, thermomechanical, and rheological characterizations of bulk glass-forming pd40ni40p20. J. Appl. Phys., 87(3):1141-1152, 2000.

[152] Graham Williams and David C Watts. Non-symmetrical dielectric relaxation behaviour arising from a simple empirical decay function. Transactions of the Faraday society, 66:80-85, 1970.

[153] M. Winterlich, G. Diezemann, H. Zimmermann, and Roland Böhmer. Microscopic origin of the nonexponential dynamics in a glassy crystal. Phys. Rev. Lett., 91:235504, h 2003.

[154] Warren Clarence Young and Richard Gordon Budynas. Roark's formulas for stress and strain, volume 7. 2002.

[155] Amanda R. Young-Gonzales, Subarna Samanta, and Ranko Richert. Dynamics of glass-forming liquids. xix. rise and decay of field induced anisotropy in the non-linear regime. The Journal of Chemical Physics, 143(10), 2015.

[156] Hai-Bin Yu, Yuansu Luo, and Konrad Samwer. Ultrastable metallic glass. Advanced Materials, pages 1-5, 2013. 
[157] Hai-Bin Yu, Ranko Richert, Robert Maaß, and Konrad Samwer. Strain induced fragility transition in metallic glass. Nature Communications, 6(7179), 2015.

[158] Hai-Bin Yu, Ranko Richert, Robert Maaß, and Konrad Samwer. Unified criterion for temperature-induced and strain-driven glass transitions in metallic glass. Phys. Rev. Lett., 115:135701, 2015.

[159] Hai-Bin Yu, Konrad Samwer, Y. Wu, and Wei Hua Wang. Correlation between $\beta$ relaxation and self-diffusion of the smallest constituting atoms in metallic glasses. Phys. Rev. Lett., 109:095508, 2012.

[160] Hai Bin Yu, Wei Hua Wang, Hai Yang Bai, and Konrad Samwer. The $\beta$-relaxation in metallic glasses. National Science Review, 1(3):429-461, 2014.

[161] Mareike Zink, Konrad Samwer, W. L. Johnson, and S. G. Mayr. Validity of temperature and time equivalence in metallic glasses during shear deformation. Physical Review $B$, 74:012201, 2006. 